\title{
CHARACTERIZATION OF IN-PLANE SHEAR PROPERTIES OF LAMINATED COMPOSITES AT HIGH STRAIN RATES
}

\author{
A Thesis by \\ Satish K.Dandayudhapani \\ Bachelor of Engineering, Madras University, India, 2003 \\ Submitted to the department of Aerospace Engineering \\ and the faculty of Graduate School of \\ Wichita State University \\ in partial fulfillment of \\ the requirements for the degree of \\ Master of Science
}

Fall 2006 


\section{CHARACTERIZATION OF IN-PLANE SHEAR PROPERTIES OF LAMINATED COMPOSITES AT HIGH STRAIN RATES}

I have examined the final copy of this thesis for form and content and recommend that it be accepted in partial fulfillment of the requirements for the degree of Master of Science, with a major in Aerospace Engineering.

Dr. K.Suresh Raju, Committee Chair

We have read this thesis and recommend its acceptance

Dr. Bert L. Smith, Committee Member

Dr. Bob Minaie, Committee Member 


\section{DEDICATION}

To my parents and friends 


\section{ACKNOWLEDGEMENTS}

I would like to present my sincere gratitude towards my advisor Dr. K.Suresh Raju for his continuous support and guidance in completing my thesis. I acquired a lot of knowledge from him during the entire course of my master's degree. He has always helped me in understanding the technical details and overcome difficulties. For his valuable help, patience and encouragement, I owe him the deepest gratitude. It's hard to express the thanks towards him in words.

I would also like to thank Dr. Bert L. Smith and Dr. Bob Minaie for reviewing my thesis and making valuable suggestions.

Most of my experiments would not have been possible without help from NIAR laboratories. I owe the deepest appreciation to my colleagues Anand Deshpande, Vivek Mariyanna, Sandeep Shetty, Juan Acosta Felipe, Manoj Varma, and Mohan Ghimire and engineers from the National Institute for Aviation Research, especially to Kim Leng Poon and Siddartha Arood for their kind assistance in carrying out experiments and helping with various applications.

I am indebted to my many colleagues and friends for providing a stimulating and fun environment to learn and grow. I wish to thank my best friends from Wichita State University; Kumar Nijagal, Ashwin Sheshadri and Divakara Basavaraju for helping me to get through difficult times and for their emotional support, entertainment and caring.

Lastly and most importantly, I want to thank my parents who have been a source of encouragement and inspiration throughout my life.

Finally, I would like to thank all the direct and indirect supports that helped me complete this thesis. 


\begin{abstract}
The in-plane shear responses of continuous fiber reinforced composite materials under high strain rates were characterized experimentally. The V-notch rail shear configuration was used for characterizing the in-plane shear behavior of Newport NB321/3k70 plain weave carbon fabric/epoxy, NCT/321/G150 Carbon Fiber Unitape/epoxy, NB321/7781 fiberglass/epoxy, Cytec PWC/T300/3KNT plain weave carbon fabric/epoxy and Fibercote 3KPW/E365 plain weave carbon fabric/epoxy systems. The testing was conducted using a servo hydraulic testing machine at nominal stroke rates ranging between $0.00083 \mathrm{in} / \mathrm{sec}$ to $500 \mathrm{in} / \mathrm{sec}$. A maximum average shear strain rate of $654 \mathrm{rad} / \mathrm{sec}$ was achieved up to shear strain levels of 0.08 radians, during the tests. The stress-strain behavior of all material systems exhibited contrasting behavior with increasing stroke rates. The stress-strain curves for all materials exhibited an asymptotic behavior for stroke rates approaching $10 \mathrm{in} / \mathrm{sec}$ and were material dependent for stroke rates exceeding $250 \mathrm{in} / \mathrm{sec}$. For Newport NB321/3k70 and NB321/7781 systems at the highest test rate, the shear strengths increased by a factor of three relative to that of the quasi-static rate, and were independent of the reinforcement type. The shear strength for Cytec PWC/T300/3KNT, Fibercote 3KPW/E365 and Newport NCT/321/G150 increased until a stroke rate of $100 \mathrm{in} / \mathrm{sec}$ and later decreased with increasing stroke rate. The failure modes for Cytec PWC/T300/3KNT, Fibercote 3KPW/E365 and Newport NCT/321/G150 were at the minimum section of the shear coupons, while for Newport NB321/3k70 and NB321/7781, failure was observed to change from a shear mode across the minimum section to a complex failure mode away from the minimum section.
\end{abstract}




\section{TABLE OF CONTENTS}

Chapter

1. INTRODUCTION 1

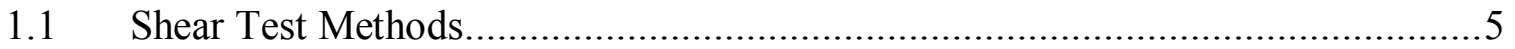

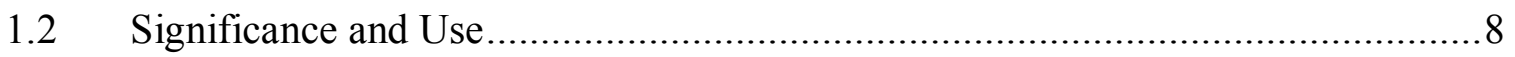

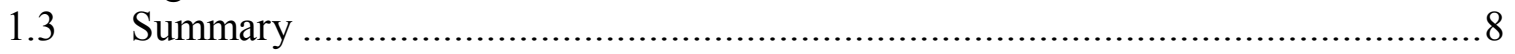

2. BACKGROUND AND LITERATURE REVIEW …........................................... 9

2.1 Background and Literature Review ...................................................... 9

2.2 Literature Review......................................................................

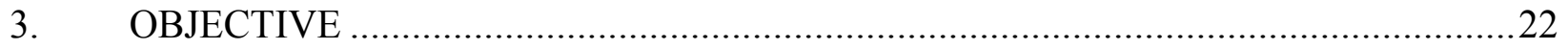

3.1 Statement of Problem................................................................................ 22

3.2 Solution Approach .................................................................................... 22

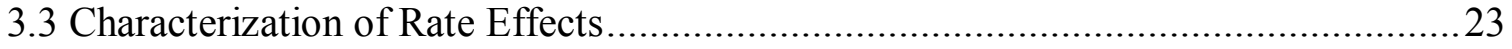

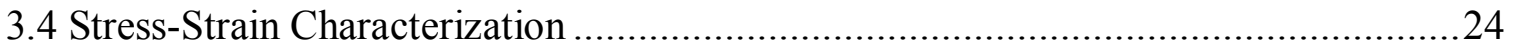

4. MATERIAL SYSTEMS AND SPECIMEN PREPARATION ..................................26

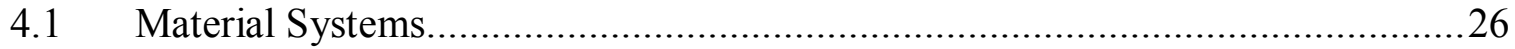

4.1.1 Laminate Production .................................................................26

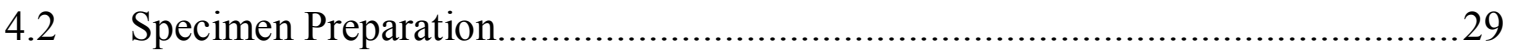

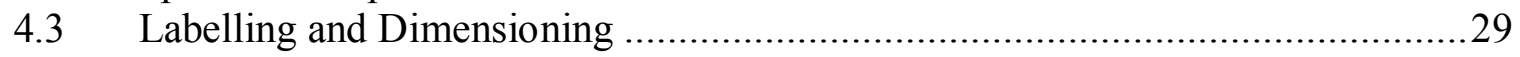

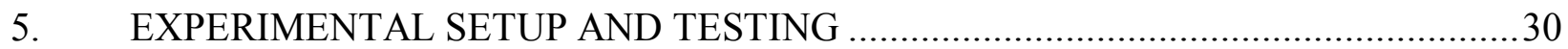

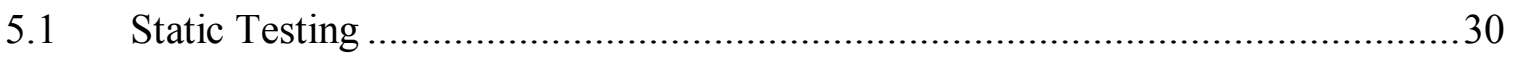

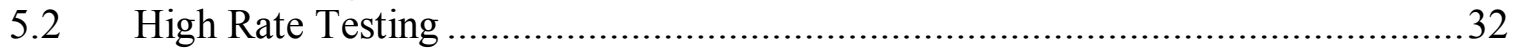

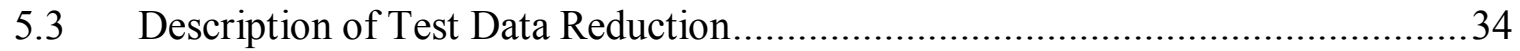

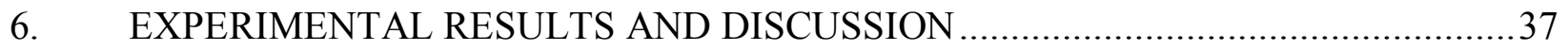

6.1 Stress-Strain Response at Quasi-Static Loading....................................... 37

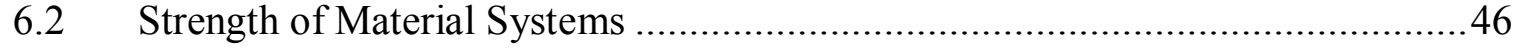

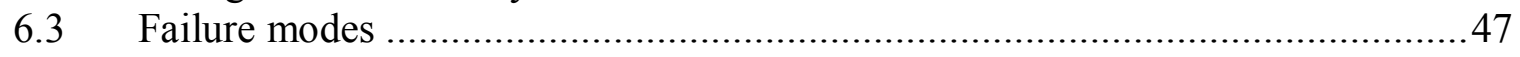

7. CONCLUSIONS AND RECOMMENDATIONS ..................................................52

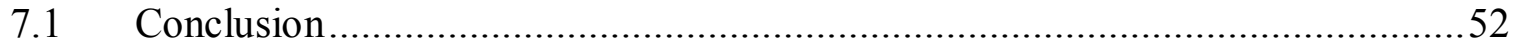

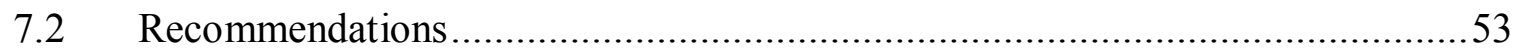




\section{TABLE OF CONTENTS}

Chapter Page

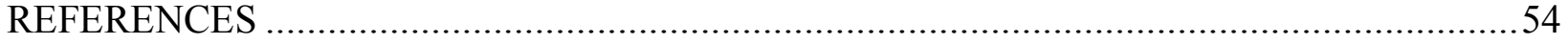

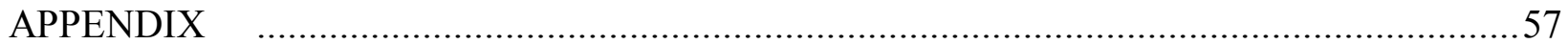




\section{LIST OF TABLES}

Table

Page

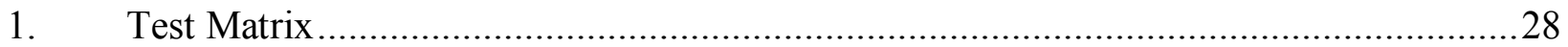

2. Summary of Test Data for Cytec PWC/T300/3KNT material.....................................41

3. Summary of Test Data for Fibercote 3KPW/E365 material ....................................42

4. Summary of Test Data for Newport NB321/3k70 PWCF material ............................42

5. Summary of Test Data for Newport NB321/7781 SWGF material .............................43

6. Summary of Test Data for Newport NCT/321/G150 material ...................................43

7. Comparison of test data for Newport SWGF material at 500in/sec .........................50

8. Summary of Test Data for Cytec PWC/T300/3KNT material...................................69

9. Summary of Test Data for Fibercote 3KPW/E365 material ...................................81

10. Summary of Test Data for Newport NB321/3k70 PWCF material ..........................93

11. Summary of Test Data for Newport NB321/7781 SWGF material ........................... 105

12. Summary of Test Data for Newport NCT/321/G150 material ................................. 117 


\section{LIST OF FIGURES}

Figure

Page

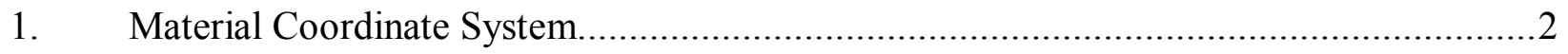

2. Orientation of Material Planes ..............................................................................

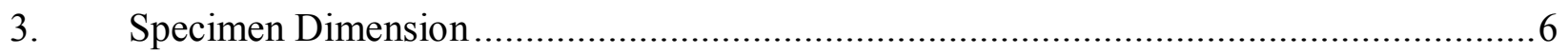

4. Partially Assembled Fixture with Specimen and Spacer Blocks ............................... 7

5. Assembled V-Notched Rail Shear Apparatus ....................................................

6. Strain Rate-Strain response of Cytec PWC/T300/3KNT at 0.00083 ...........................23 in/sec stroke rate.

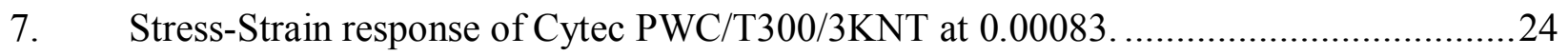
in/sec stroke rate.

8. Cure cycle for Newport NB321/3k70 PWCF, Newport NB321/7781 SWGF ..............27 and Newport NCT/321/G150 Unitape

9. Cure Cycle for Cytec PWC/T300/3KNT .............................................................22

10. Cure Cycle for Fibercote 3KPW/E365 ........................................................28

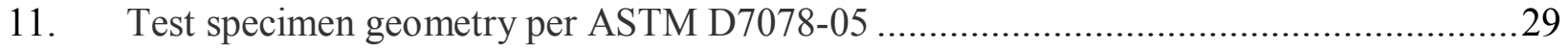

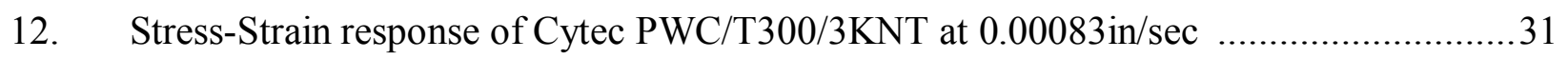
stroke rate

13. Strain Rate-Strain response of Cytec PWC/T300/3KNT at $0.00083 \mathrm{in} / \mathrm{sec} \ldots \ldots \ldots \ldots \ldots . . . . . . . .31$ stroke rate

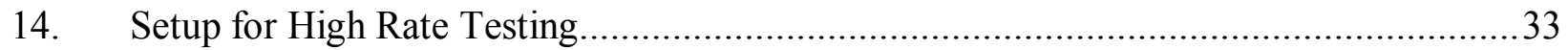

15. Time lag between strain and stress signals during a high-rate test ...........................35

16. Strain-Time data for Cytec PWC/T300/3KNT at 250in/sec stroke rate..........................36 


\section{LIST OF FIGURES (continued)}

Figure $\quad$ Page

17. Shear stress-Displacement diagram for the materials tested at quasi static test rate.........37

18. Shear stress-shear strain diagram for the materials tested at quasi static test rate ............38

19. In-plane shear stress-shear strain behavior of Cytec PWC/T300/3KNT ......................39 material at different stroke rates

20. In-plane shear stress-shear strain behavior of Fibercote 3KPW/E365 .........................39 material at different stroke rates

21. In-plane shear stress-shear strain behavior of Newport NB321/3k70 PWCF ..............40 material at different stroke rates

22. In-plane shear stress-shear strain behavior of Newport NB321/7781 ........................40 SWGF material at different stroke rates

23. In-plane shear stress-shear strain behavior of Newport NCT/321/G150. Unitape material at different stroke

24. In-plane shear stress- strain at $100 \mathrm{in} / \mathrm{sec}$

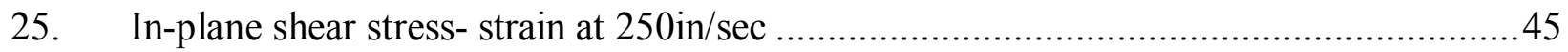

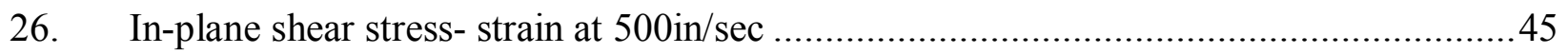

27. Strength of the material systems at different stroke rates ..................................... 46

28. Average Strain Rates of material systems at different stroke rates..........................47

29. Failure modes in Cytec PWC/T300/3KNT material at different stroke rates.................48

30. Failure modes in Fibercote 3KPW/E365 material at different stroke rates ...................48

31. Failure modes in Newport NCT/321/G150 Unitape material ................................48 at different stroke rates 
Figure

\section{LIST OF FIGURES (continued)}

32. Failure modes in Newport NB321/7781 SWGF material at different stroke rates...........49

33. Failure modes in Newport NB321/3k70 PWCF material ........................................49 at different stroke rates

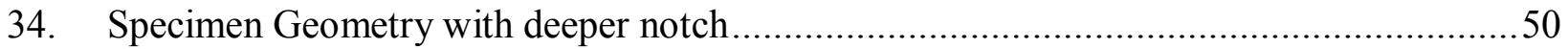

35. Comparison of shear stress-strain between two gage lengths................................51

36. Comparison of strain rate-strain between two gage lengths ......................................51

37. Stress-Strain and Strain Rate-Strain behavior of Cytec PWC/T300/3KNT .................58 at $0.00083 \mathrm{in} / \mathrm{s}$ stroke rate

38. Stress-Strain and Strain Rate-Strain behavior of Cytec PWC/T300/3KNT .58 at $0.00083 \mathrm{in} / \mathrm{s}$ stroke rate

39. Stress-Strain and Strain Rate-Strain behavior of Cytec PWC/T300/3KNT at $0.00083 \mathrm{in} / \mathrm{s}$ stroke rate

40. Stress-Strain and Strain Rate-Strain behavior of Cytec PWC/T300/3KNT at $1 \mathrm{in} / \mathrm{s}$ stroke rate

41. Stress-Strain and Strain Rate-Strain behavior of Cytec PWC/T300/3KNT 60 at $1 \mathrm{in} / \mathrm{s}$ stroke rate

42. Stress-Strain and Strain Rate-Strain behavior of Cytec PWC/T300/3KNT 60 at $1 \mathrm{in} / \mathrm{s}$ stroke rate

43. Stress-Strain and Strain Rate-Strain behavior of Cytec PWC/T300/3KNT 61 at $10 \mathrm{in} / \mathrm{s}$ stroke rate

44. Stress-Strain and Strain Rate-Strain behavior of Cytec PWC/T300/3KNT 61 at $10 \mathrm{in} / \mathrm{s}$ stroke rate 
Figure

\section{LIST OF FIGURES (continued)}

45. Stress-Strain and Strain Rate-Strain behavior of Cytec PWC/T300/3KNT at $10 \mathrm{in} / \mathrm{s}$ stroke rate

46. Stress-Strain and Strain Rate-Strain behavior of Cytec PWC/T300/3KNT at $100 \mathrm{in} / \mathrm{s}$ stroke rate

47. Stress-Strain and Strain Rate-Strain behavior of Cytec PWC/T300/3KNT .63 at $100 \mathrm{in} / \mathrm{s}$ stroke rate

48. Stress-Strain and Strain Rate-Strain behavior of Cytec PWC/T300/3KNT at $100 \mathrm{in} / \mathrm{s}$ stroke rate

49. Stress-Strain and Strain Rate-Strain behavior of Cytec PWC/T300/3KNT 64 at $250 \mathrm{in} / \mathrm{s}$ stroke rate

50. Stress-Strain and Strain Rate-Strain behavior of Cytec PWC/T300/3KNT .64 at $250 \mathrm{in} / \mathrm{s}$ stroke rate

51. Stress-Strain and Strain Rate-Strain behavior of Cytec PWC/T300/3KNT .65 at $250 \mathrm{in} / \mathrm{s}$ stroke rate

52. Stress-Strain and Strain Rate-Strain behavior of Cytec PWC/T300/3KNT .65 at $500 \mathrm{in} / \mathrm{s}$ stroke rate

53. Stress-Strain and Strain Rate-Strain behavior of Cytec PWC/T300/3KNT .66 at $500 \mathrm{in} / \mathrm{s}$ stroke rate

54. Failure mode of Cytec PWC/T300/3KNT material at $0.00083 \mathrm{in} / \mathrm{sec}$ .66

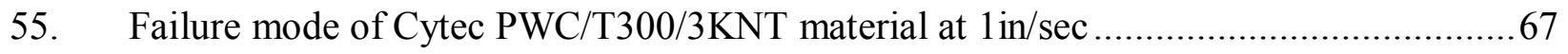

56. Failure mode of Cytec PWC/T300/3KNT material at 10in/sec ...............................67

57. Failure mode of Cytec PWC/T300/3KNT material at 100in/sec .67 
Figure

\section{LIST OF FIGURES (continued)}

58. Failure mode of Cytec PWC/T300/3KNT material at 250in/sec .............................68

59. Failure mode of Cytec PWC/T300/3KNT material at 500in/sec ...............................68

60. Stress-Strain and Strain Rate-Strain behavior of Fibercote 3KPW/E365 .................... 70 at $0.00083 \mathrm{in} / \mathrm{s}$ stroke rate

61. Stress-Strain and Strain Rate-Strain behavior of Fibercote 3KPW/E365 .................... 70 at $0.00083 \mathrm{in} / \mathrm{s}$ stroke rate

62. Stress-Strain and Strain Rate-Strain behavior of Fibercote 3KPW/E365 .71 at $0.00083 \mathrm{in} / \mathrm{s}$ stroke rate

63. Stress-Strain and Strain Rate-Strain behavior of Fibercote 3KPW/E365 .71 at $1 \mathrm{in} / \mathrm{s}$ stroke rate

64. Stress-Strain and Strain Rate-Strain behavior of Fibercote 3KPW/E365 at $1 \mathrm{in} / \mathrm{s}$ stroke rate

65. Stress-Strain and Strain Rate-Strain behavior of Fibercote 3KPW/E365 .................... 72 at $1 \mathrm{in} / \mathrm{s}$ stroke rate

66. Stress-Strain and Strain Rate-Strain behavior of Fibercote 3KPW/E365 .73 at $10 \mathrm{in} / \mathrm{s}$ stroke rate

67. Stress-Strain and Strain Rate-Strain behavior of Fibercote 3KPW/E365 .73 at $10 \mathrm{in} / \mathrm{s}$ stroke rate

68. Stress-Strain and Strain Rate-Strain behavior of Fibercote 3KPW/E365 .74 at $10 \mathrm{in} / \mathrm{s}$ stroke rate

69. Stress-Strain and Strain Rate-Strain behavior of Fibercote 3KPW/E365 at $100 \mathrm{in} / \mathrm{s}$ stroke rate 
Figure

\section{LIST OF FIGURES (continued)}

70. Stress-Strain and Strain Rate-Strain behavior of Fibercote 3KPW/E365 ................... 75 at $100 \mathrm{in} / \mathrm{s}$ stroke rate

71. Stress-Strain and Strain Rate-Strain behavior of Fibercote 3KPW/E365 .................... 75 at $100 \mathrm{in} / \mathrm{s}$ stroke rate

72. Stress-Strain and Strain Rate-Strain behavior of Fibercote 3KPW/E365 ....................76 at $250 \mathrm{in} / \mathrm{s}$ stroke rate

73. Stress-Strain and Strain Rate-Strain behavior of Fibercote 3KPW/E365 .76 at $250 \mathrm{in} / \mathrm{s}$ stroke rate

74. Stress-Strain and Strain Rate-Strain behavior of Fibercote 3KPW/E365 77 at $250 \mathrm{in} / \mathrm{s}$ stroke rate

75. Stress-Strain and Strain Rate-Strain behavior of Fibercote 3KPW/E365 at $500 \mathrm{in} / \mathrm{s}$ stroke rate

76. Stress-Strain and Strain Rate-Strain behavior of Fibercote 3KPW/E365 .................... 78 at $500 \mathrm{in} / \mathrm{s}$ stroke rate

77. Stress-Strain and Strain Rate-Strain behavior of Fibercote 3KPW/E365 ................... 78 at $500 \mathrm{in} / \mathrm{s}$ stroke rate

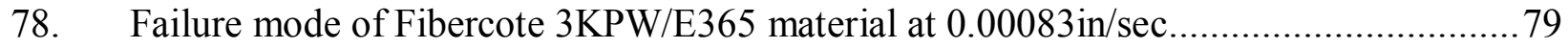

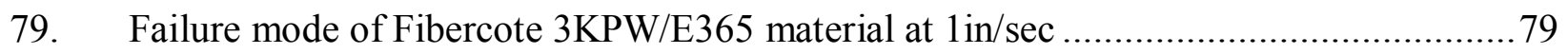

80. Failure mode of Fibercote 3KPW/E365 material at 10in/sec ..................................79

81. Failure mode of Fibercote 3KPW/E365 material at 100in/sec ................................. 80

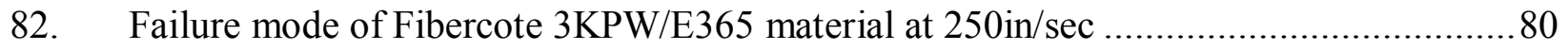

83. Failure mode of Fibercote 3KPW/E365 material at 500in/sec .................................80 
Figure

\section{LIST OF FIGURES (continued)}

84. Stress-Strain and Strain Rate-Strain behavior of Newport NB321/3k70 PWCF 82 at $0.00083 \mathrm{in} / \mathrm{s}$ stroke rate

85. Stress-Strain and Strain Rate-Strain behavior of Newport NB321/3k70 PWCF .82 at $0.00083 \mathrm{in} / \mathrm{s}$ stroke rate

86. Stress-Strain and Strain Rate-Strain behavior of Newport NB321/3k70 PWCF .83 at $1 \mathrm{in} / \mathrm{s}$ stroke rate

87. Stress-Strain and Strain Rate-Strain behavior of Newport NB321/3k70 PWCF at $1 \mathrm{in} / \mathrm{s}$ stroke rate

88. Stress-Strain and Strain Rate-Strain behavior of Newport NB321/3k70 PWCF .84 at $1 \mathrm{in} / \mathrm{s}$ stroke rate

89. Stress-Strain and Strain Rate-Strain behavior of Newport NB321/3k70 PWCF....... at $10 \mathrm{in} / \mathrm{s}$ stroke rate

90. Stress-Strain and Strain Rate-Strain behavior of Newport NB321/3k70 PWCF .85 at $10 \mathrm{in} / \mathrm{s}$ stroke rate

91. Stress-Strain and Strain Rate-Strain behavior of Newport NB321/3k70 PWCF .85 at $10 \mathrm{in} / \mathrm{s}$ stroke rate

92. Stress-Strain and Strain Rate-Strain behavior of Newport NB321/3k70 PWCF. .86 at $100 \mathrm{in} / \mathrm{s}$ stroke rate

93. Stress-Strain and Strain Rate-Strain behavior of Newport NB321/3k70 PWCF .86 at $100 \mathrm{in} / \mathrm{s}$ stroke rate

94. Stress-Strain and Strain Rate-Strain behavior of Newport NB321/3k70 PWCF .87 at $100 \mathrm{in} / \mathrm{s}$ stroke rate 
Figure

\section{LIST OF FIGURES (continued)}

95. Stress-Strain and Strain Rate-Strain behavior of Newport NB321/3k70 PWCF 87 at $250 \mathrm{in} / \mathrm{s}$ stroke rate

96. Stress-Strain and Strain Rate-Strain behavior of Newport NB321/3k70 PWCF 88 at $250 \mathrm{in} / \mathrm{s}$ stroke rate

97. Stress-Strain and Strain Rate-Strain behavior of Newport NB321/3k70 PWCF .88 at $250 \mathrm{in} / \mathrm{s}$ stroke rate

98. Stress-Strain and Strain Rate-Strain behavior of Newport NB321/3k70 PWCF 89 at $500 \mathrm{in} / \mathrm{s}$ stroke rate

99. Stress-Strain and Strain Rate-Strain behavior of Newport NB321/3k70 PWCF .89 at $500 \mathrm{in} / \mathrm{s}$ stroke rate

100. Stress-Strain and Strain Rate-Strain behavior of Newport NB321/3k70 PWCF .90 at $500 \mathrm{in} / \mathrm{s}$ stroke rate

101. Failure mode of Newport NB321/3k70 PWCF material at $0.00083 \mathrm{in} / \mathrm{sec}$ .90

102. Failure mode of Newport NB321/3k70 PWCF material at $1 \mathrm{in} / \mathrm{sec}$ 91

103. Failure mode of Newport NB321/3k70 PWCF material at 10in/sec..............................91

104. Failure mode of Newport NB321/3k70 PWCF material at 100in/sec............................91

105. Failure mode of Newport NB321/3k70 PWCF material at 250in/sec...........................92

106. Failure mode of Newport NB321/3k70 PWCF material at 500in/sec...........................92

107. Stress-Strain and Strain Rate-Strain behavior of Newport NB321/7781 SWGF .94 at $0.00083 \mathrm{in} / \mathrm{s}$ stroke rate

108. Stress-Strain and Strain Rate-Strain behavior of Newport NB321/7781 SWGF. .94 at $0.00083 \mathrm{in} / \mathrm{s}$ stroke rate 
Figure

\section{LIST OF FIGURES (continued)}

109. Stress-Strain and Strain Rate-Strain behavior of Newport NB321/7781 SWGF. .95 at $0.00083 \mathrm{in} / \mathrm{s}$ stroke rate

110. Stress-Strain and Strain Rate-Strain behavior of Newport NB321/7781 SWGF. 95 at $1 \mathrm{in} / \mathrm{s}$ stroke rate

111. Stress-Strain and Strain Rate-Strain behavior of Newport NB321/7781 SWGF. .96 at $1 \mathrm{in} / \mathrm{s} \mathrm{stroke} \mathrm{rate}$

112. Stress-Strain and Strain Rate-Strain behavior of Newport NB321/7781 SWGF. .96 at $1 \mathrm{in} / \mathrm{s}$ stroke rate

113. Stress-Strain and Strain Rate-Strain behavior of Newport NB321/7781 SWGF...... .97 at $10 \mathrm{in} / \mathrm{s}$ stroke rate

114. Stress-Strain and Strain Rate-Strain behavior of Newport NB321/7781 SWGF. at $10 \mathrm{in} / \mathrm{s}$ stroke rate

115. Stress-Strain and Strain Rate-Strain behavior of Newport NB321/7781 SWGF. .98 at $10 \mathrm{in} / \mathrm{s}$ stroke rate

116. Stress-Strain and Strain Rate-Strain behavior of Newport NB321/7781 SWGF. .98 at $100 \mathrm{in} / \mathrm{s}$ stroke rate

117. Stress-Strain and Strain Rate-Strain behavior of Newport NB321/7781 SWGF. .99 at $100 \mathrm{in} / \mathrm{s}$ stroke rate

118. Stress-Strain and Strain Rate-Strain behavior of Newport NB321/7781 SWGF. .99 at $100 \mathrm{in} / \mathrm{s}$ stroke rate

119. Stress-Strain and Strain Rate-Strain behavior of Newport NB321/7781 SWGF. 100 at $250 \mathrm{in} / \mathrm{s}$ stroke rate 
Figure

\section{LIST OF FIGURES (continued)}

120. Stress-Strain and Strain Rate-Strain behavior of Newport NB321/7781 SWGF ............. 100 at $250 \mathrm{in} / \mathrm{s}$ stroke rate

121. Stress-Strain and Strain Rate-Strain behavior of Newport NB321/7781 SWGF 101 at $250 \mathrm{in} / \mathrm{s}$ stroke rate

122. Stress-Strain and Strain Rate-Strain behavior of Newport NB321/7781 SWGF. 101 at $500 \mathrm{in} / \mathrm{s}$ stroke rate

123. Stress-Strain and Strain Rate-Strain behavior of Newport NB321/7781 SWGF. 102 at $500 \mathrm{in} / \mathrm{s}$ stroke rate

124. Failure mode of Newport NB321/7781 SWGF material at $0.00083 \mathrm{in} / \mathrm{sec}$ 102

125. Failure mode of Newport NB321/7781 SWGF material at $1 \mathrm{in} / \mathrm{sec}$ 103

126. Failure mode of Newport NB321/7781 SWGF material at $10 \mathrm{in} / \mathrm{sec}$

127. Failure mode of Newport NB321/7781 SWGF material at 100in/sec. 103

128. Failure mode of Newport NB321/7781 SWGF material at 250in/sec. 104

129. Failure mode of Newport NB321/7781 SWGF material at 500in/sec. 104

130. Stress-Strain and Strain Rate-Strain behavior of Newport NCT/321/G150 106 Unitape at $0.00083 \mathrm{in} / \mathrm{s}$ stroke rate

131. Stress-Strain and Strain Rate-Strain behavior of Newport NCT/321/G150 106 Unitape at $0.00083 \mathrm{in} / \mathrm{s}$ stroke rate

132. Stress-Strain and Strain Rate-Strain behavior of Newport NCT/321/G150 107 Unitape at $1 \mathrm{in} / \mathrm{s}$ stroke rate

133. Stress-Strain and Strain Rate-Strain behavior of Newport NCT/321/G150 107 Unitape at $1 \mathrm{in} / \mathrm{s}$ stroke rate 
Figure

\section{LIST OF FIGURES (continued)}

134. Stress-Strain and Strain Rate-Strain behavior of Newport NCT/321/G150 _..................108

Unitape at $1 \mathrm{in} / \mathrm{s}$ stroke rate

135. Stress-Strain and Strain Rate-Strain behavior of Newport NCT/321/G150

108

Unitape at $10 \mathrm{in} / \mathrm{s}$ stroke rate

136. Stress-Strain and Strain Rate-Strain behavior of Newport NCT/321/G150

Unitape at $10 \mathrm{in} / \mathrm{s}$ stroke rate

137. Stress-Strain and Strain Rate-Strain behavior of Newport NCT/321/G150

Unitape at $10 \mathrm{in} / \mathrm{s}$ stroke rate

138. Stress-Strain and Strain Rate-Strain behavior of Newport NCT/321/G150

Unitape at 100in/s stroke rate

139. Stress-Strain and Strain Rate-Strain behavior of Newport NCT/321/G150

Unitape at $100 \mathrm{in} / \mathrm{s}$ stroke rate

140. Stress-Strain and Strain Rate-Strain behavior of Newport NCT/321/G150

Unitape at 100in/s stroke rate

141. Stress-Strain and Strain Rate-Strain behavior of Newport NCT/321/G150

Unitape at $250 \mathrm{in} / \mathrm{s}$ stroke rate

142. Stress-Strain and Strain Rate-Strain behavior of Newport NCT/321/G150

Unitape at $250 \mathrm{in} / \mathrm{s}$ stroke rate

143. Stress-Strain and Strain Rate-Strain behavior of Newport NCT/321/G150

Unitape at $250 \mathrm{in} / \mathrm{s}$ stroke rate

144. Stress-Strain and Strain Rate-Strain behavior of Newport NCT/321/G150

Unitape at 500in/s stroke rate 
Figure

\section{LIST OF FIGURES (continued)}

145. Stress-Strain and Strain Rate-Strain behavior of Newport NCT/321/G150 113

Unitape at 500in/s stroke rate

146. Stress-Strain and Strain Rate-Strain behavior of Newport NCT/321/G150

Unitape at 500in/s stroke rate

147. Failure mode of Newport NCT/321/G150 Unitape material at $0.00083 \mathrm{in} / \mathrm{sec}$

148. Failure mode of Newport NCT/321/G150 Unitape material at $1 \mathrm{in} / \mathrm{sec} . \ldots \ldots \ldots \ldots \ldots \ldots \ldots \ldots . . . . .115$

149. Failure mode of Newport NCT/321/G150 Unitape material at 10in/sec......................115

150. Failure mode of Newport NCT/321/G150 Unitape material at 100in/sec.....................115

151. Failure mode of Newport NCT/321/G150 Unitape material at 250in/sec....................116

152. Failure mode of Newport NCT/321/G150 Unitape material at 500in/sec.....................116 


\section{CHAPTER 1 \\ INTRODUCTION}

In recent years, composite materials are increasingly replacing conventional metallic materials in airframe and automotive structures. Some applications of composite materials involve dynamically loaded components and structures. Hence, there is a need to fully understand the dynamic behavior of composite materials to facilitate the analysis and design of composite structures under high strain rate loadings. Research activity performed in this area has several purposes. First, there is a need to understand the effects of strain rates on the material behavior, strength, stiffness and failure modes, as limited experiments on composite structures under impact loading have exhibited characteristics that are contrasting relative to that at quasistatic rates. Secondly, there is an increasing demand for numerical modeling of structures subjected to high rate loading, which requires reliable dynamic material properties and complete stress-strain curves as a function of strain rate.

As the number and diversity of applications for fiber- reinforced composite materials continue to increase, the need for new and improved test methods continues. One area of continuing test method development is shear testing to measure the shear strengths and shear moduli of these composites. Shear testing is most commonly performed to measure the in-plane shear properties of a composite material: the in-plane shear modulus, the in-plane shear strength, or both. The inplane shear is shear associated with shear forces or deformation applied to the 1-2 material plane such that the resulting shear deformations occur in the plane of the laminate. Any of the shear properties describing the response resulting from a shear load or deformation applied to the 1-3 
or 2-3 material planes is the interlaminar shear. A cartesian coordinate system describing the principal material coordinate system using 1, 2, and 3 for the axes, as shown in Figure 1.

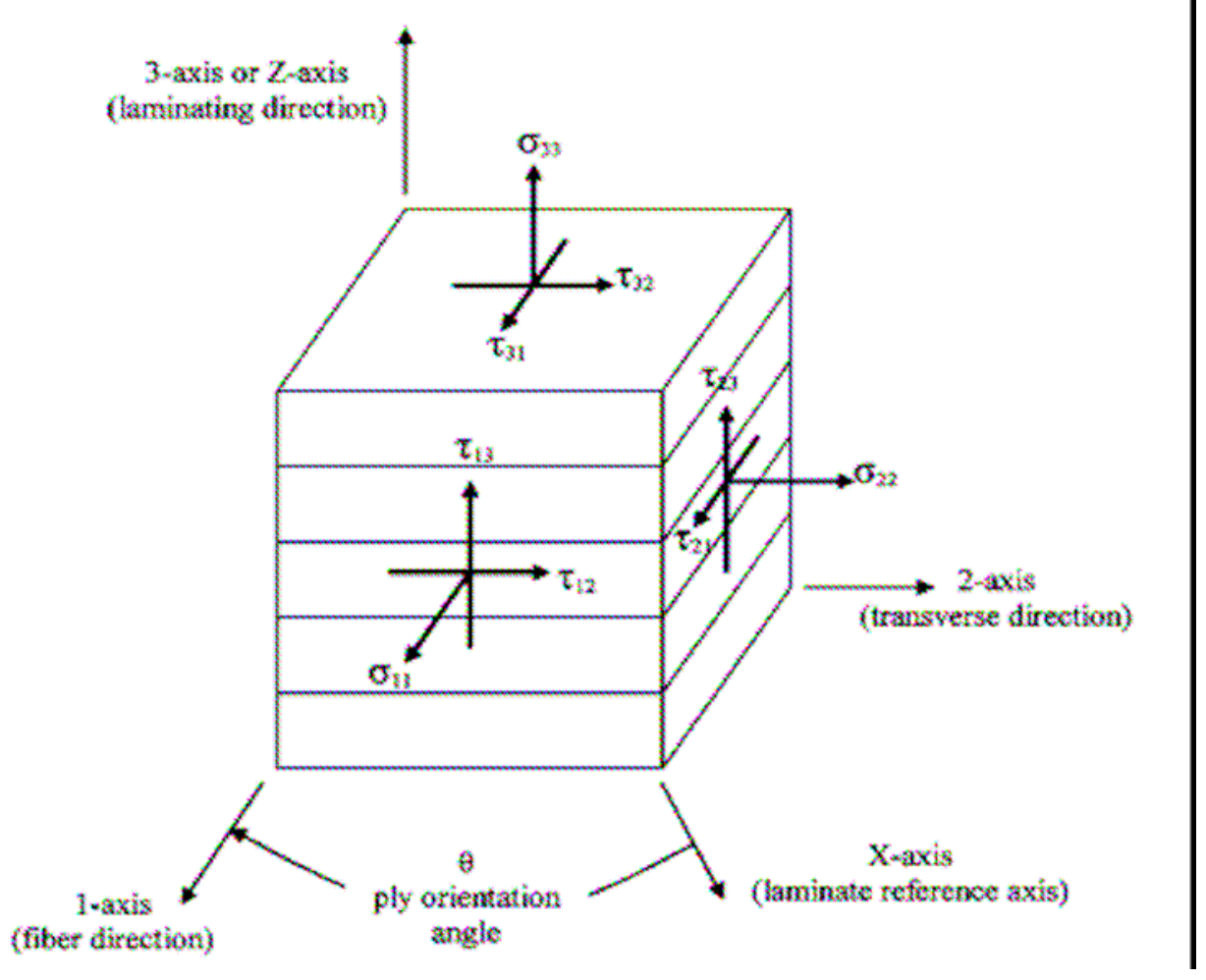

Figure 1. Material Coordinate System [1]

An intrinsically low resistance to shear deformation, particularly in material planes dominated by matrix properties, is a severe weakness in fiber reinforced plastic composites. Relatively low values of shear stiffness and strength often compromise material performance, forcing designers to arrange laminate stacking sequences in order to maximize shear resistance. The resultant effect of shear property optimization is that mechanical properties are frequently compromised. Small tangential stresses can lead to severe reductions in the load bearing capacity of composite structures; hence the needs for accurate methods for measuring shear properties. Considerable experimental and analytical effort has been expended in the development of in-plane and through thickness (out-of-plane) shear test methods for the determination of shear modulus and strength 
of fiber reinforced polymer composites. One of the principal difficulties in the development of a test method for the measurement of shear properties is the provision of a pure shear stress state in the specimen [2]. Ideally, for quantitative shear measurements, the shear stress must be uniform in the test section of the specimen throughout the linear and non linear response regimes. This region should be one of the maximum shear stress relative to all other regions of the specimen. In addition, a unique relationship should exist between the applied load and the magnitude of the shear stress in the test section.

The difficulty of inducing pure shear increases with increasing anisotropy and inhomogeneity of the material. As these characteristics increase, the complex stress states arising at or near the loading zones become more dominant, particularly for continuous unidirectional laminates containing high modulus and high strength fibers. In these materials, it is difficult to obtain adequate regions of uniform shear stress free of extraneous stress components within the specimen, even if the production of the specimen and the test alignment were perfect. In addition, extraneous tensile and compressive stress components have a marked effect on the shear strength of these materials [2]. Tensile stresses induce premature failure, where as compressive stresses delay the onset of failure. The difficulties encountered in producing a state of pure shear in composite specimens have resulted in a limited number of these methods being incorporated into national and international standards. There is no universal method suitable for the accurate evaluation of the shear properties for the extensive range of the material architectures encountered in composite technology [2]. All the shear methods standardized or otherwise, have physical and geometrical limitations [2]. Most shear tests have been developed with the objective of maximizing shear stress and minimizing extraneous induced stresses. It is possible to measure the shear stress-strain response of a composite in the presence of non-shear 
stresses, provided the magnitude of the shear stress is considerably larger than the other stress contributions. Acceptable shear moduli measurements can be obtained from a range of test methods. Full characterization of the shear properties of a composite lamina or laminate requires the measurement of shear modulus and shear strength in the 1-2, 1-3 and 2-3 planes (Figure 2). Because in-plane(1-2) and interlaminar shear properties(1-3,2-3) are not necessarily equal, test methods have been developed to induce both in-plane and interlaminar shear loading. Here, we are only concerned about in-plane shear test methods.
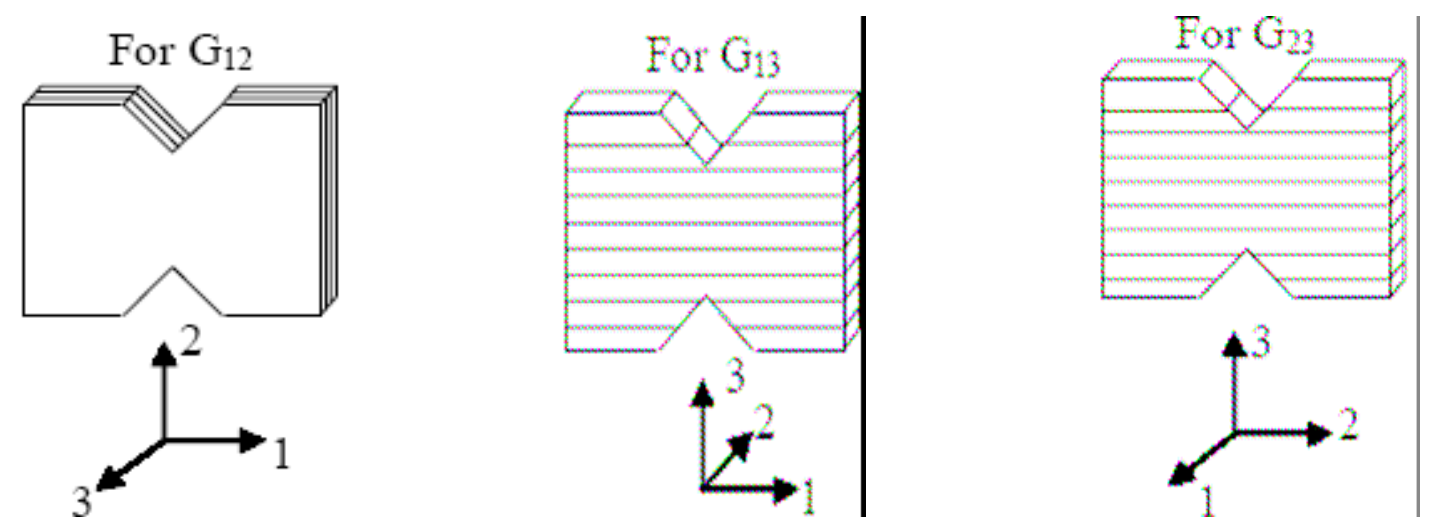

Figure 2. Orientation of Material Planes [1]

High strain rate characterization of polymer composite materials has been limited due to difficulties in the measuring equipment. In particular, jigs and fixtures can be subject to inertial disturbances present at speeds up to and above $78.74 \mathrm{in} / \mathrm{s}$ [3]. These disturbances are due to the phenomena of mechanical resonance and control problems that the test equipment experiences at high speeds. The amplitudes of the resulting oscillations increase with test speed and change the test sample response, making results difficult to analyze. These inertial problems may be avoided if the extrapolation of low to high strain data is applicable. 


\subsection{Shear Test Methods:}

As the number and diversity of applications for fiber-reinforced composite materials continue to increase, the need for new and improved test methods continues. One area of continuing test method development is shear testing to measure the shear strengths and shear moduli of these composites. Shear testing is most commonly performed to measure the in-plane shear properties of a composite material: the in-plane shear modulus, the in-plane shear strength, or both. Depending on the composite material to be tested and which material properties are desired, a particular shear test method may be preferred relative to others. Although several test methods have been developed to address these needs, no single shear test is universally accepted as the preferred method for obtaining in-plane or interlaminar shear properties of composite materials [2]. Currently, the most popular of these shear test methods are the Iosipescu (V-notched) shear test ASTM D5379, the two-rail shear test ASTM D4255/D4255M, the V-notched rail shear test ASTM D7078/D7078M, the short beam shear test ASTM D2344/D2344M, and the $\pm 45^{\circ}$ tensile shear test ASTM D3518/D 3518M.

The test method ASTM D7078/D7078M method was chosen to study the shear properties of laminated composite materials in this investigation because of the tensile loading mode. The tensile loading mode wherein the two fixture halves move away from each other is safer under high rates of testing. A material coupon in the form of a flat rectangle with symmetrical centrally located V-notches, shown schematically in Figure 3, is clamped with two fixture halves (pictured in Figure 4, and shown schematically in Figure 5 and in more detail in the machining drawings of ASTM adjunct ADJD7078). When loaded in tension using a mechanical testing machine, this fixture introduces shear forces in the 
specimen that produce failures across the notched specimen. The specimen is inserted into the two fixture halves with the notches aligned with the line of the applied load. The two halves of the assembled fixture are extended by a testing machine while monitoring load. The relative displacement between the two fixture halves produces shear stresses in the notched specimen. By placing two strain gage elements, oriented at $\pm 45^{\circ}$ to the loading axis, in the middle of the specimen and along the loading axis, the shear strain response of the material can be measured.

The notches influence the shear strain distribution in the central region of the coupon, producing a more uniform distribution than without notches. As a result of the reduced specimen width due to the notches, the average shear stress is increased relative to the unnotched width.
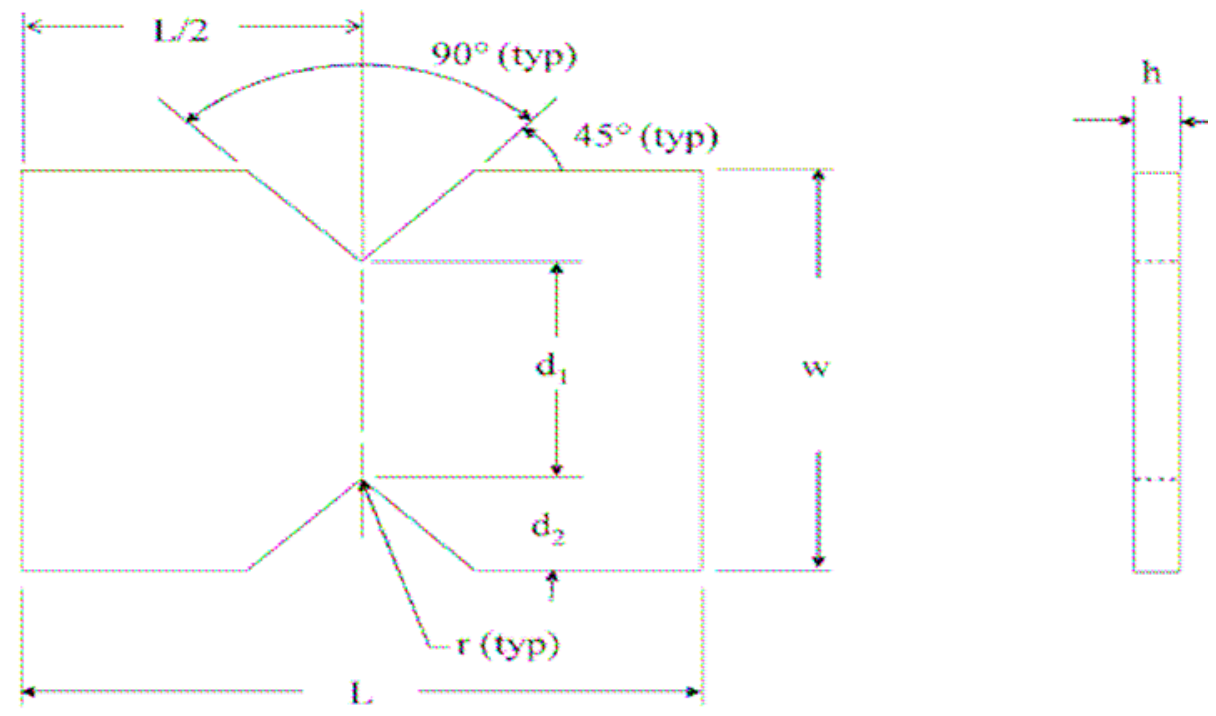

Front

End

Nominal Specimen Dimensions

$$
\begin{aligned}
& \mathrm{d}_{1}=31.0 \mathrm{~mm}[1.20 \mathrm{in.}] \\
& \mathrm{d}_{2}=12.7 \mathrm{~mm}[0.50 \mathrm{in.}] \\
& \mathrm{h}=\text { as required } \\
& \mathrm{L}=76.0 \mathrm{~mm}[3.0 \mathrm{in} .] \\
& \mathrm{r}=1.3 \mathrm{~mm}[0.05 \mathrm{in.}] \\
& \mathrm{w}=56.0 \mathrm{~mm}[2.20 \mathrm{in.}]
\end{aligned}
$$

Figure 3. Specimen Dimensions [1] 


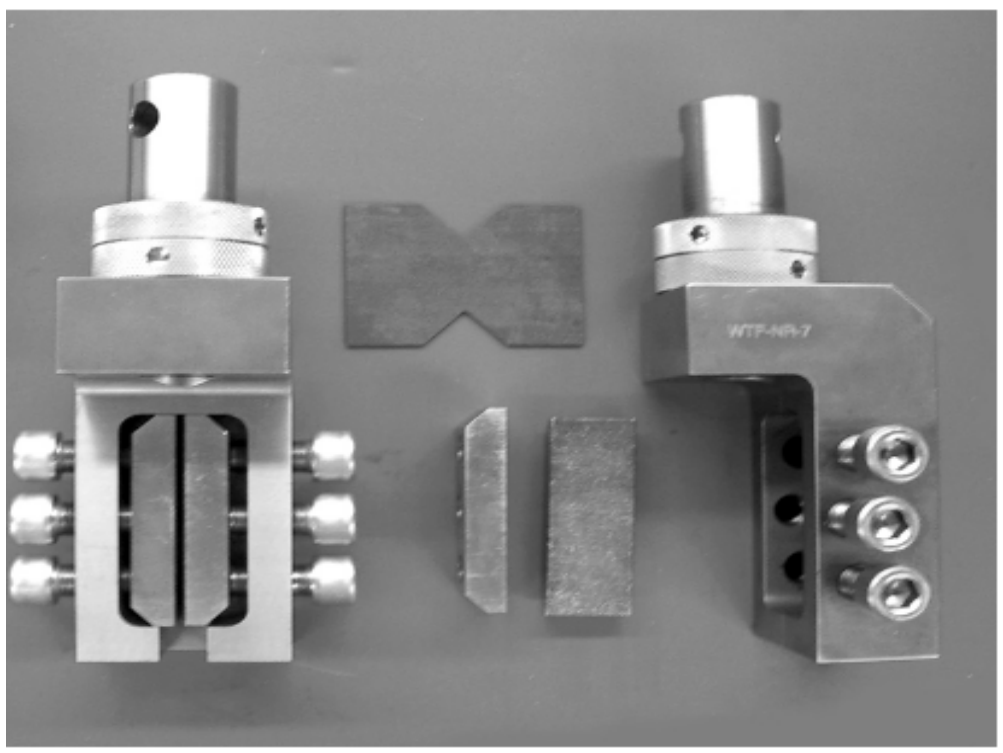

Figure 4. Partially Assembled Fixture with Specimen and Spacer Blocks [1]

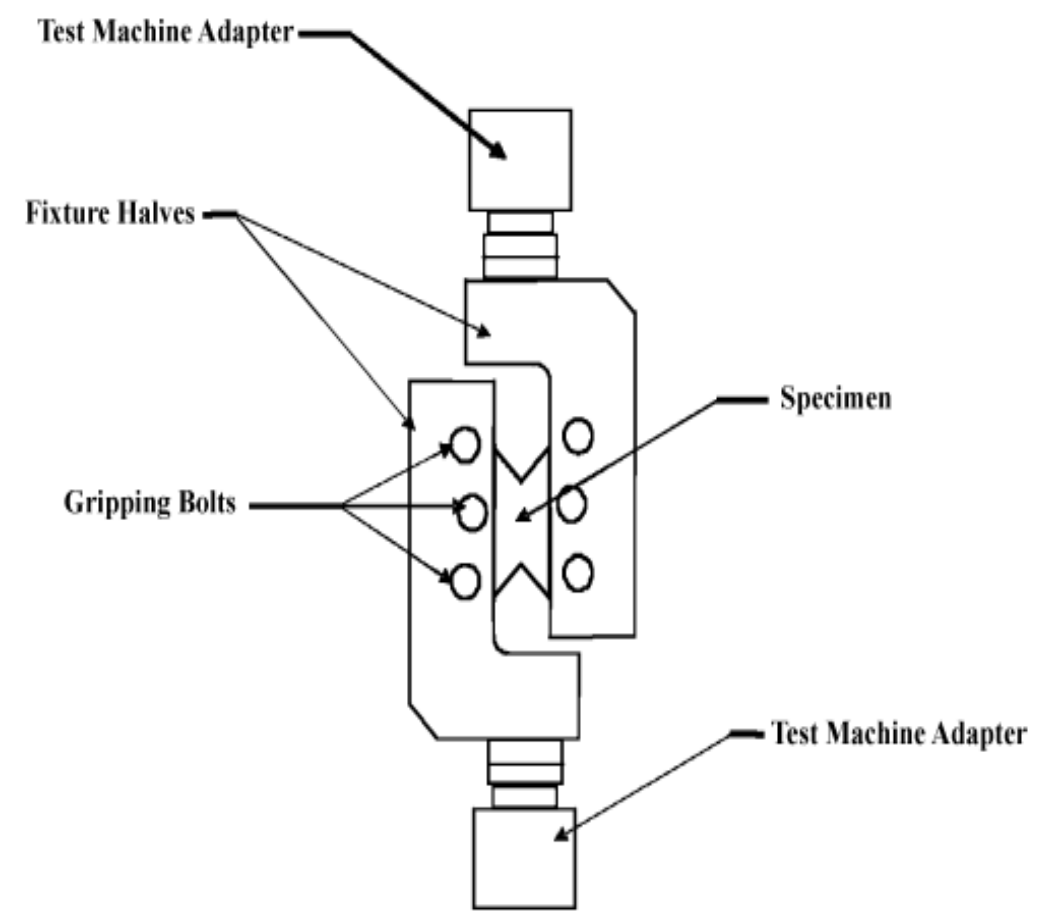

Figure 5. Assembled V-Notched Rail Shear Apparatus [1] 


\subsection{Significance and Use:}

This shear test is designed to produce shear property data for material specifications, research and development, quality assurance, and structural design and analysis. Either in-plane or interlaminar shear properties may be evaluated, depending upon the orientation of the material coordinate system relative to the loading axis. Factors that influence the shear response and should therefore be reported include: material, methods of material preparation and lay-up, specimen stacking sequence, specimen preparation, specimen conditioning, environment of testing, specimen alignment and gripping, speed of testing, time at temperature, void content, and volume percent reinforcement. In anisotropic materials, properties may be obtained in any of the six possible shear planes by orienting the testing plane of the specimen with the desired material plane (1-2 or $2-1,1-3$ or $3-1,2-3$ or $3-2)$. Only a single shear plane may be evaluated for any given specimen.

\subsection{Summary:}

This investigation involves the characterization of in-plane shear properties of laminated composite materials at high strain rates where the data containing the basic material properties like ultimate shear strength, modulus, nature of stress-strain curves and strain rate variation over the test time is to be obtained. The study of failure modes at different stroke rates is one of the major objectives. Five different materials were studied; test coupons with notches were manufactured as per ASTM D7078-05. Tests are conducted at quasi-static rate of 0.00083in/sec on a 22kip MTS machine and at five different higher stroke rates of $1 \mathrm{in} / \mathrm{s}, 10 \mathrm{in} / \mathrm{s}, 100 \mathrm{in} / \mathrm{s}$, 250in/s and 500in/s on a special purpose high speed machine. The experimental observations and results are discussed in this report. 


\section{CHAPTER 2}

\section{BACKGROUND AND LITERATURE REVIEW}

\subsection{Background and Literature Review:}

Shear tests are performed for several reasons. The results of these tests are used for selecting the proper materials for engineering applications. Shear properties are measured during development of new materials so that new materials can be developed and compared. Shear properties of composites are often measured to predict the material behavior under loading conditions. Strength of the materials is always primary concern in shear tests. High strain rate shear testing is necessary to understand the material response under dynamic loading conditions. Strain rates ranging from $10^{2} \mathrm{rad} / \mathrm{sec}$ to $10^{4} \mathrm{rad} / \mathrm{sec}$ can occur in many practical events like foreign object impact damage, blast loadings, structural impacts etc. The behavior of materials under high strain rate shear loads may be considerably different than that of static loadings. The determination of composite shear properties is an important but delicate issue. Such are shear moduli, complete stress-strain responses, and shear strengths.

\subsection{Literature Review:}

Kammerer and Neme [4] investigated the impact behavior of a ceramic/composite bilayered target, the high strain rate behavior of an E-glass/Polyester composite. A static characterization of the ply in tensile and plane shear with different strain rates (from $10^{-5}$ to $10^{-3}$ /s) were carried out. They proposed new system for testing orthotropic materials with the tensile Hopkinson-bar apparatus. The high strain rate testing was carried out on E-glass/polyester specimens and simulated with constitutive models. Simulations and the experiments provided good results. From the results it was possible to identify the behavior of other quasi- 
unidirectional organic matrix composites, different from the E-glass/polyester, with the same elasto-viscoplastic 'bi-material' constitutive law.

Andrew M. Myer and Anthony J. Vizzini [5] studied the effect of strain rate on interlaminar shear properties. Analysis of the reason for the increase in shear strength shows a correlation to the crack propagation rate of the composite material. The crack along the shear face starts as the stress concentration at the notch end causes an initial delamination. The delamination at the two notch ends propagate towards one another causing the failure. Once the crack is initiated, the delamination extends along the shear face. This leads to the conclusion that the shear strength relies on the propagation rate of the crack through the resin-rich area of the laminate. Therefore the interlaminar failure and the rate of the delamination are driven by the resin behavior of the composite. In addition, the viability of double notch shear specimens for high strain rate analysis using a Split Hopkinson Pressure Bar was analyzed. Due to the secondary failure that occurs at higher strain rates, the Double Notch Shear (DNS) test fails as a precise test specimen as there is a much less precision as the strain rate rises. The test gave an excellent response at lower strain rates on a traditional load cell, but as higher impact rates were achieved, the consistency in failure modes could not be maintained leading to imprecision in the data analysis. Changes in geometry such as increasing the shear area could help increase the test range of strain rates but that would result in larger specimens. For purposes of maintaining a common specimen shape and precision, this change was not performed in this research.

B.Bouette et al., [6] investigated the effect of strain rate on interlaminar shear properties of laminates. The material tested was T300/5208 Carbon/Epoxy composite, the range of strain rates explored was about $10^{-3}$ to $10^{3} / \mathrm{sec}$. The specimens used by the authors were designed and optimized by finite element analysis. One of the specimens permitted the determination of the 
interlaminar shear modulus, $\mathrm{G}_{13}$, and the other permitted the determination of the interlaminar shear strength, $\mathrm{S}_{13}$. The specimens were tested at different speeds at low, intermediate and high strain rates. The authors found peeling and shear stress distributions in the shear zone of the specimen by finite element calculations, which were used to evaluate the effect of the overlap length and shear height on these contributions, and also used to compute the interlaminar shear modulus, $\mathrm{G}_{13}$. This calculated modulus was compared with that measured experimentally and the agreement was satisfactory. Finally, the authors optimized the specimen geometry in order to obtain the interlaminar shear modulus and fracture stress by direct measurement. The experiments were used in determining the interlaminar shear properties of the material at low, intermediate and high strain rates. They observed no strain rate effect on the material studied, which was a unidirectional T300/5208 carbon/epoxy composite. They detected a slight difference in the fracture surfaces under the scanning electron microscope, a greater density of resin debris and fiber fractures was noted on the specimens tested at high strain rate.

Papadakis, et al., [7] investigated the characterization of the shear mechanical properties of glass-fiber reinforced thermoplastic composite laminates over a range of strain rates. Twenty two $[45]_{2 \mathrm{~s}}$ specimens each were tested at 5,50 and $500 \mathrm{~mm} / \mathrm{min}$ cross head displacement rates, using a universal testing machine. The longitudinal and transverse strains were obtained experimentally using extensometer apparatus and then transformed to the ply axis using classical laminate theory. A rigorous statistical treatment method was proposed for the processing and analysis of the raw data. The shear modulus decreased for increasing strain rate. The shear failure stress increased for increasing strain rate. Semi-empirical linear functions of the shear modulus and shear failure strength were proposed with respect to the logarithm of the shear strain rate. The shear failure strain was independent of strain rate. Finally, the observed opposing 
trends of in-plane shear modulus and shear failure stress suggested that shear damage evolution is strain rate dependent for the examined material. The proposed methodology applied to the plytron material over three orders of the magnitude of crosshead displacement rate yielded discernable strain rate effects in some properties. The shear modulus of plytron decreases for increasing strain rate. The authors proposed the linear model of the shear modulus with respect to the logarithm of shear strain rate. The individual results for the shear modulus and shear strength properties demonstrate that for the examined material shear damage evolution is strain rate dependent.

I.W. Hall and M. Guden [8] conducted high strain rate testing of a unidirectionally reinforced graphite epoxy composite. High strain rate tests were conducted using a compression Split Hopkinson Pressure Bar (SHPB) with 19mm diameter Inconel-718 bars. Two different striker bars were used with stress pulse time-windows of 150 and $290 \mu$ s. A major concern for composites is the time for stress homogenization in the specimen. For isotropic high impedance ceramic materials, stress homogenization is achieved after approximately $4 t_{o}$ where $t_{o}$ is the transit time for the leading edge of the pulse traveling through the specimen. Even for nonaxisymmetric loading of unidirectionally reinforced composites, stress homogenization is still achieved after 3 or 4 reflections. In order to be even more certain of stress homogenization in the present work, some of the experiments were performed using pulse shapers to produce a gradually increasing compressive stress pulse. In addition, some samples were strain gaged in order to compare the stress/strain curves with those produced by conventional SHPB data reduction routines. Another problem of SHPB testing is crushing of the tested sample by further movement of the bars after failure. Instead of using a momentum trap to prevent crushing after failure, in the present work loosely-fitting high strength steel collars were placed around the 
samples. The collars were prepared $5-7 \%$ shorter than the sample and permitted recovery of tested material by preventing further deformation after fracture. In the transverse direction, the fracture strength increased noticeably from $215 \mathrm{MPa}$ at quasi-static strain rate to an approximately constant value of $360 \mathrm{MPa}$ at high strain rate. The fracture strain was almost constant at $5 \pm 0.3 \%$, and no significant change was noted in Young's modulus over the strain rate range investigated. In the longitudinal direction there was considerably more scatter in the data but only a slight indication of a strain rate dependence as the fracture strength increased from $850 \mathrm{MPa}$ to $950 \mathrm{MPa}$. The variability in the data for the longitudinal data is ascribed to the effect of slight misalignment of the fibers in and between the prepreg sheets. The modulus showed a slight decline from $90 \mathrm{GPa}$ down to $75 \mathrm{GPa}$ over the strain rate range but the fracture strain remained constant at $2 \pm 0.3 \%$. The decline in modulus is unusual in so far as increases in moduli have been reported elsewhere. The reasons for this discrepancy are still under investigation. The results indicate strong strain rate dependence of the strength properties in the transverse direction but no similar dependence longitudinally and the reasons for this behavior may be deduced from the fracture surfaces. Since the only significant event occurring in the transverse direction is failure of the epoxy matrix, this must be the source of the strain rate sensitivity noted. Longitudinally, the fibers themselves undergo extensive buckling, followed by larger-scale kinking as a precursor to failure of the fiber/matrix interface. Buckling and kinking require, of course, failure at the fiber/matrix interface but the stress required must be of the order of that required to cause failure at this location in transverse samples, i.e. $360 \mathrm{MPa}$. Since this is well below the intrinsic compressive strength of the fiber bundles and it does not contribute to the strain rate sensitivity in this case. It is concluded that high strain rate mechanical properties of polymer matrix composites can be reliably and reproducibly measured using the SHPB provided 
that care is taken to ensure stress homogenization. Furthermore, these particular composite exhibits only strain rate sensitivity of the fracture strength, and this only in the transverse direction.

R. Garcia, et al., [9] investigated experimentally and analytically the rail shear-test method as applied to composite materials. The effects of nonuniform stresses induced by differential thermal expansion, rail flexibility and specimen aspect ratio on measured shear modulus and ultimate strength of composite laminates were shown. A two dimensional linearly elastic finite-element model was used to analytically determine how various geometric parameters influenced the magnitude and distribution of in-plane normal and shear stresses in a tensile rail-shear specimen. Rail shear tests were conducted at room temperature and $589 \mathrm{~K}$ on selected graphite-polyimide composite laminates using two titanium rail configurations. An analytical and experimental study has been presented on the effects of mechanical and thermal loading on the shear modulus and ultimate-strength results obtained from the rail shear-test procedure. The test specimens studied were four graphite-polyimide laminates tested at room temperature and 589K. A two-dimensional linearly elastic finite-element model was used to explore the influence of thermal loadings and various geometric parameters on the magnitude and distribution of in-plane normal and shear stresses in the rail shear specimen. The major observations from this study were the direction of loading, axial or diagonal has little effect on stresses within the specimen. The aspect ratio of the specimen can have a major effect on stress distributions, and this varies according to the laminate. Tapering the rails resulted in slight changes in the shear-stress distribution but increased the magnitude of the normal stresses. Very high normal stresses occurred perpendicular to the filaments in the 0 -deg oriented specimen and, in fact, initiate premature failures relative to the 90-deg specimen. Because of better gripping of 
the specimen, the bonded rail-test configuration provided better stiffness and ultimate-strength data than the bolted configuration at room temperature. Since processing techniques for bonding rails at $589 \mathrm{~K}$ were not available, the bolted-rail test technique was used for the elevatedtemperature tests. Results of these tests indicated that an accurate measure of the initial shear modulus can be obtained and, as with the room-temperature tests, the measured ultimate strengths are conservative.

Ahmed K.Hussain and Donald F.Adams [10] conducted linear three dimensional finite element analyses to evaluate various rail and specimen configurations of two rail shear test procedure. Additional analytical evaluations were performed to investigate the effects of specimen aspect ratio, material orientation, loading direction, and the rigidity of the rails on stress distributions. Shear stresses were found to be distributed uniformly over a large region in the specimen gage section for most of the models analyzed, but normally accompanied by significant transverse and axial normal stresses. The results indicated that an axial loading applied to a test fixture of uniform steel rails and a test specimen with the aspect ratio of 6 was the preferred test procedure. Simple test specimens of trapezoidal and rectangular geometries, and specimens with tabs, were chosen for further evaluation. Also, the new Wyoming-modified two-rail shear test fixture was established based on these analyses. Criteria for use in the design of a new shear test fixture were obtained from the detailed finite element analysis study presented here. A variety of specimen and rail configurations were analyzed. In addition, several other parameters that were expected to affect the results were also studied. Significant amounts of information were obtained through this analytical evaluation of candidate test specimen geometries. After several candidate configurations have been chosen, a nonlinear three dimensional analysis using WYO3D, or a failure initiation and propagation analysis using the 
recently modified WYO2D program, can be performed. As an alternative, experimental evaluation was conducted using only a small number of different configurations. Authors concluded experimental evaluation is often the best approach since analytical methods do not always accurately predict the response in an actual test environment. A large central region of uniform shear stress along with a low transverse tensile stress was obtained for most of the finite element analysis models. Therefore, authors strongly believed that the [90] and [0] specimens are capable of providing reliable shear modulus values. However, a [0/90] cross-ply specimen should provide an accurate measure of both the shear modulus and shear strength of a composite material.

Konrad Gliesche, et al., [11] studied shear properties of carbon/epoxy composites with $0^{\circ} / 90^{\circ}$ textile reinforcement structures and different layer thickness under $+/-45^{\circ}$ tensile loading. Deformation process on specimen surfaces during loading was studied using an optical whole field measuring method. The results show that the shear behavior was affected by the layer thickness and/or by the weight per unit area of the reinforcement. The optical deformation analysis of the samples surfaces did not show an equal strain distribution, as expected. Moreover, large deviations from the calculated average strain values were obtained. As consequence the dimensioning of construction units influenced by this fact. It was found a correlation between shear properties, single layer thickness and the optical measured deformation inhomogeneity at the surface. The woven fabric-220 with the finest layer thickness showed the best shear properties and the smallest strain inhomogeneity. TFT-interleaved acts as woven fabric-395 which consisted of thicker layers. The non-crimped fabric with the largest layer thickness showed the lowest shear properties and the highest strain inhomogeneity. Authors explained the lowest shear properties with the layer thickness but the great strain inhomogeneity on the surface 
not. It was assumed that the yarn cut-ins influence there deformation properties. It was necessary to investigate non-woven structures with thinner layers. The pictures of the optical strain measurements did not show constant strain for all examined structures over the specimen surface. The effect is to recognize for both longitudinal and transverse extension. These strain fields showed an optical $45^{\circ}$ structure. For all load levels, the sectional diagrams demonstrated that the strain inhomogeneity exists already at the smallest load level whereby their peaks arise with loading, but they were stationary along the specimen surfaces and there was stating that also the reinforcement structure effect this inhomogeneity. In some papers, on model specimens such inhomogeneity was investigated, in the residual interlaminar deformations was proposed to measure to the cooled down state of CRF epoxy composite with a micro -moiré interferometer. The different ply orientations in the laminates were taken. The strain measurement was carried out at the specimen surface edgewise. The results showed that the measured strains were not constant through the thickness, as authors expected. The derivations occur at the resin interfaces which are especially high between the $0^{\circ} / 45^{\circ}$ plies. Weikl has reported about strain inhomogeneity at the specimen surfaces and he described the possibility to detect delamination defects coming from the inside of the laminates at the specimen surfaces with an optical method. This defect affected displacements at the surface and was connected with a correlation between internal defects and exterior strain derivations. The author proposed the optical measurement method to non-destructively check of coupons with regard to defects.

Gary L. Farley and Donald J. Baker [12] studied the in-plane shear test of thin panels; an improved shear fixture for determination of in-plane shear response of thin composite panels has been designed, fabricated and verified. Finite-element analyses were conducted to aid in fixture design. Adverse corner stresses, tearing and crimping failures inherent in commonly used shear 
fixtures were minimized. The analyses indicate that location of the fixture corner pins was the most significant parameter in reducing the corner-stress problems. Secondary parameters that contributed to a uniform shear-stress distribution were the relative stiffness of the loading tabs compared to the panel stiffness and the distance between the fixture corner pins and the fixturepanel load-transfer bolts. Test results for thin panels indicate that classical diagonal-tension fields were developed and tension failures initiated across a buckle near a panel corner. Correlation of experimental data with analysis indicates that diagonal-tension theory can be used to predict the load-strain response of thin-composite panels.

A.K. Hussain and D.F. Adams [13] evaluated the Wyoming-modified two rail Shear Test method for composite materials. In-plane unidirectional shear properties of carbon/epoxy and glass/epoxy materials were measured using unidirectional and cross-ply specimens utilizing the Wyoming modified two-rail shear test fixture. Simple test specimens of trapezoidal and rectangular geometries and specimens with tabs were tested. Various specimen aspect ratios were also considered. Untabbed unidirectional specimens exhibited premature shear failures but cross-ply specimens produced reasonably reliable results with acceptable failure modes. Both bonded tab and integral tab $0 \sim$ specimens were found to give comparable results. Unidirectional and cross-ply PEEK specimens, and angle-ply and quasi-isotropic AS4/3501-6 specimens, were also tested. The Wyoming-modified two-rail shear test fixture has been shown to produce reasonable in-plane shear property data. An untabbed cross-ply laminate is a highly recommended rectangular specimen configuration for use with the fixture. As an alternative, a tabbed $0 \sim$ unidirectional specimen can be considered, but this requires a more laborious specimen fabrication procedure since tabs must be adhesively bonded to the specimen. Hence this is not as attractive. A few [0] rectangular specimens were found to produce shear strengths 
comparable to the $[0 / 90]$ rectangular configuration. More of these specimens need to be tested, and more strain gage test data are needed. Perhaps with these additional data, a sound definition of shear strength can be established. Another specimen configuration worthy of further exploration is rectangular with notches. From the experimental evaluation, this specimen was found to provide the highest shear strengths for [0] unidirectional specimens.

H.Ho, et al., [11] studied in-plane shear testing of graphite-woven fabric composites. The Iosipescu shear test method was used to determine the in-plane shear response of AS4 and celion carbon fiber/epoxy fabric composite materials. Several weave architectures were studied: AS4 uniweave, AS4 and Celion plain weaves, Celion 5-harness and 8-harness satin weaves. Specimens were tested using traditional strain gage techniques and full-field moiré interferometry. A full-node localized hybrid analysis is introduced to perform efficient reduction data, producing whole-field strain distributions in the specimen test section. It was found that the fabric yarn size greatly influenced the uniformity of the shear field in the specimen test section. However, consistent shear moduli still can be obtained using the modified Iosipescu specimen and Wyoming fixture except for fabrics with large fiber yarns. The shear response of five woven composite materials was evaluated experimentally using the Iosipescu shear test. In addition to the inherent twisting problems associated with shear testing of composite materials with high transverse stiffness, the strain distribution between the notches was highly nonuniform for all fabric materials evaluated due to the nature of weave architecture. The overall shear response of the woven fabric composite is attributed to the combination of the global (due to the textile architecture of the specimen) and fluctuating local (due to the textile architecture of the surface layer) behaviors. Localized high shear strains were found at resin rich regions between the fiber bundles. However, consistent shear modulus can be obtained for woven fabric composites except 
for large tow sized fabrics. Strain measurements using strain gages are not accurate for fabrics having large filament count yams due to the profound local nonuniformity effect. The overall issues of testing and summary for various strain rate ranges are discussed here. These strain rates are generally covered by universal test instruments and servo-hydraulic machines. Slack grip system is used on servo-hydraulic machines to ramp to the needed test speed prior to engaging the test specimen. Specimen geometry details, force and strain measurement details and data collection and data reduction issues are elaborated.

Recent regulations by the government regarding automobile/aircraft safety issues [15] have served as motivation for the development of high strain rate test data to observe the changes in the material properties in case of a crash event. This data is required as inputs for computational design models. If accurate numerical results can be obtained from the computational models and high strain rate test data, the need to conduct expensive crash tests may be dramatically reduced. This not only translates into significant cost savings, but also will allow the manufacturers to make minor adjustments in their designs in a more efficient manner. However, generating high rate shear data is much more complicated than generating static shear data. The assumptions generally true in static shear tests do not always apply during high strain rate testing. Additionally, a great deal of engineering judgement and interpretation is required when reducing high strain rate data. Because of this, the shear data obtained from the high strain rate test specimens should only be compared with data obtained from tests having similar experimental setups and data reduction methods.

Composite manufacturers and users would like to directly compare their results with other published data on the same or similar material. Because high strain rate data is very sensitive to 
specimen geometry and test and analysis techniques, directly comparing test data without having a full understanding of how that data was generated is not valid. 


\section{CHAPTER 3}

\section{OBJECTIVE}

\subsection{Statement of Problem}

The objective of this investigation is to identify the high strain rate shear testing variables that may have a significant effect on the resulting data and to suggest a method that specifically addresses the issues related to high strain rate shear testing of composites. Making it simple, the purpose of shear testing is to determine ultimate shear stress and shear modulus of the material. With the crashworthiness perspective, the following aspects of the composite laminated coupons in static and high strain rate shear loadings were to be studied:

1. The ultimate shear strength of the material.

2. Stress-Strain response.

3. Failure pattern of the test coupons according to the strain rates.

4. Nature of the strain rate with respect to the strain throughout the test.

\subsection{Solution Approach}

Using MTS test systems, the specimens are tested in static and high stroke rate shear loadings. A 22 Kip MTS hydraulic machine is used for all static tests and a special purpose high rate MTS hydraulic machine is used for all high rate tests. Tests are conducted at quasi-static rate of $0.00083 \mathrm{in} / \mathrm{sec}$ and at five different higher stroke rates of $1 \mathrm{in} / \mathrm{s}, 10 \mathrm{in} / \mathrm{s}, 100 \mathrm{in} / \mathrm{s}, 250 \mathrm{in} / \mathrm{s}$ and $500 \mathrm{in} / \mathrm{s}$. For each stroke rate including the static test, 3 specimens each are tested, for each material. The test data obtained in each test is analyzed for strain rate behavior, stress-strain response and ultimate shear strength. 


\subsection{Strain Rate and Stroke Rate:}

Until now many researchers have done extensive work in the area of shear testing of polymer composites [7]. Unlike metals there are no specified, material properties rate sensitive documented for polymer composites. Until now, past researchers suggested many test methods to determine the specific material properties, so that one can use those to implement in a finite element code to reduce the extensive work of testing for individual material configurations.

Based on the strain gage signal, it can be inferred that the shear strain rate varies linearly with time, at least up to the shear strain value of 0.08 radians (from Figure 6). A constant shear strain rate test could be conducted by using the strain signal as a feedback, but will be limited by the maximum strain that can be measured by strain gages.

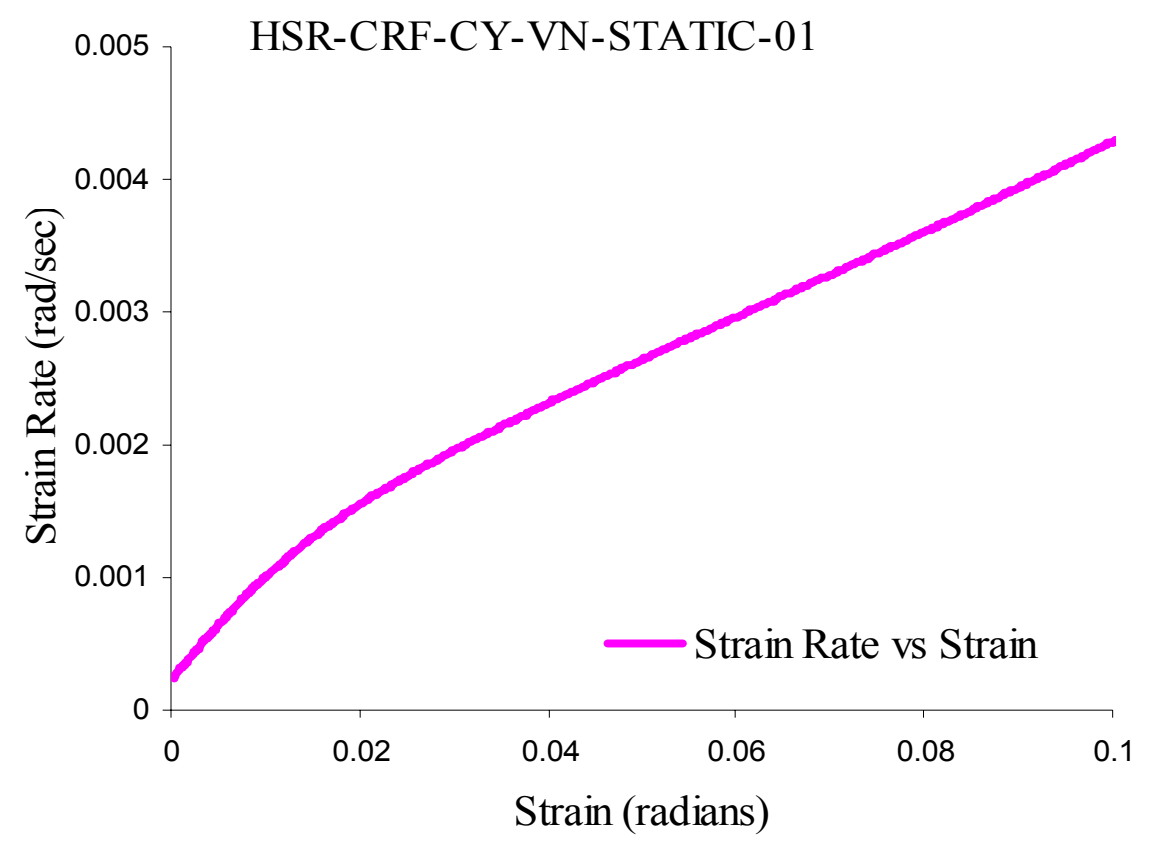

Figure 6. Strain Rate-Strain response of Cytec PWC/T300/3KNT at $0.00083 \mathrm{in} / \mathrm{sec}$ stroke rate. 
Further, the constant strain-rate test will require the actuator to decelerate to compensate for the increasing strain rate, which would be difficult to achieve at higher stroke rates. In addition, the flexibility of elements forming the load train between the specimen and the actuator contribute to the non-uniformity of stroke rate at the specimen fixture. Thus in this investigation, the term 'constant stroke rate' refers to the actuator speed and not the relative displacement between the fixture halves.

\subsection{Stress-Strain Characterization:}

The stress-strain response is one of the objectives in this investigation. The stress-strain diagram (shown in the Figure 7) is truncated at a shear strain value of 0.1 radians, which is the limit of the strain gage.

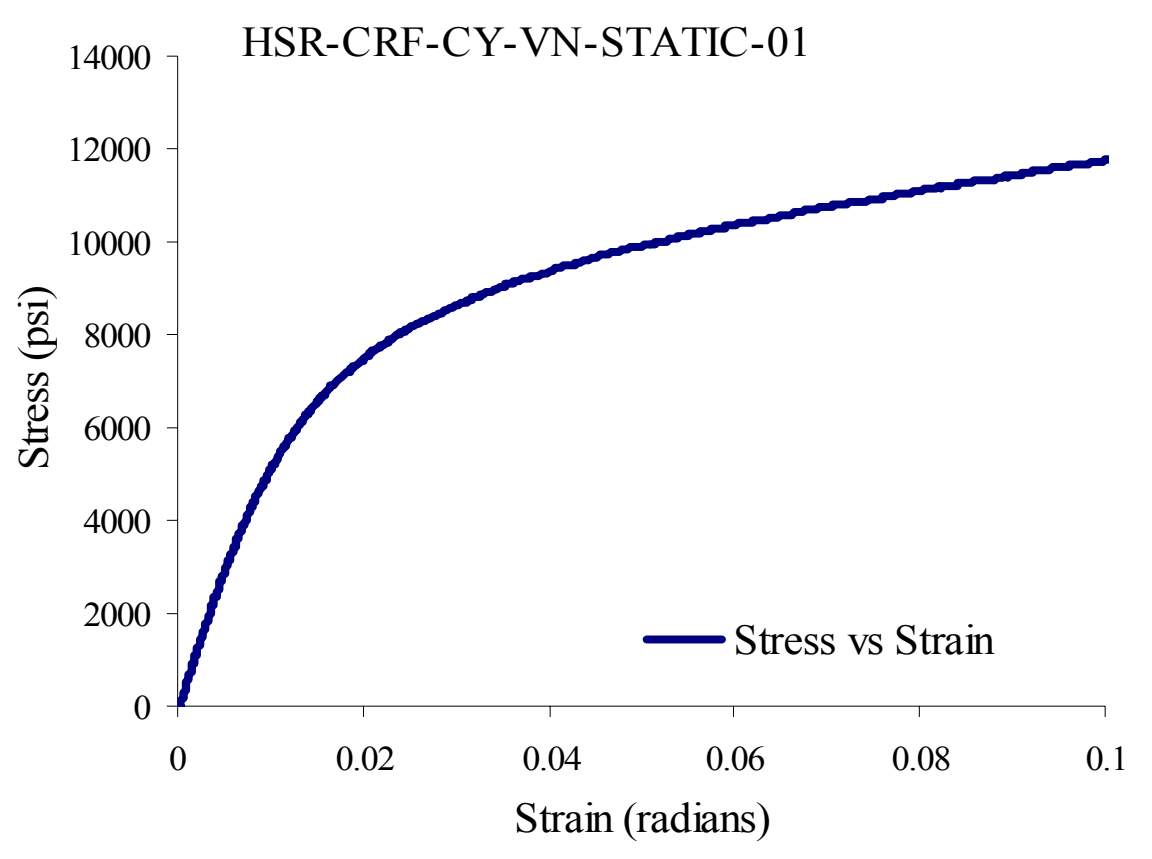

Figure 7. Stress-Strain response of Cytec PWC/T300/3KNT at $0.00083 \mathrm{in} / \mathrm{sec}$ stroke rate.

The test specimens can sustain load beyond this strain limit and the maximum strain value recorded during the test is not considered as the failure strain. It should be noted that the shear 
strains are obtained from the normal strains measured along two orthogonal directions, using transformation equations based on small-strain theory. Owing to small normal strains along the length of the specimen(x-direction) due to constraint effects, and assuming uniform shear strain distribution over the area covered by the strain gage, the error will be less than $0.5 \%$. 


\section{CHAPTER 4}

\section{MATERIAL SYSTEMS AND SPECIMEN PREPARATION}

\subsection{Material Systems:}

The material systems used in current investigation were Newport NB321/3K70 P plain weave carbon epoxy prepreg (PWCF) [16], NB321/7781 fiberglass/epoxy prepreg (SWGF) [16], NCT/321/G150 Carbon Fiber Unitape [16], Cytec PWC/T300/3KNT plain weave carbon fabric epoxy [17], and Fibercote 3KPW/E365 plain weave carbon fabric epoxy [18] systems. All the material systems share the same resin system with different cure cycles as shown in the table.

\subsubsection{Laminate Production:}

Thin sheets, known as laminates, are manufactured from the layers of fibres preimpregnated with partially cured resin prepreg. The matrix is usually an epoxy. All the material systems share the same resin system, the prepreg are cut into plies each measuring 48inches x 12 inches with stacking sequence of $[0]_{12}$ from the reel and placed top of each other with the fibers in each layer oriented relative to one another in a predetermined sequence. The layers are carefully smoothed out to prevent air entrapment. It is essential that the layers are aligned with reference to a datum, since even a few degrees misalignment can cause a dramatic effect on mechanical properties. Following completion of the lay up, the stack of prepreg layers is prepared for curing in an autoclave. Figures 8, 9, 10 illustrates the Curing cycles for all material systems. 


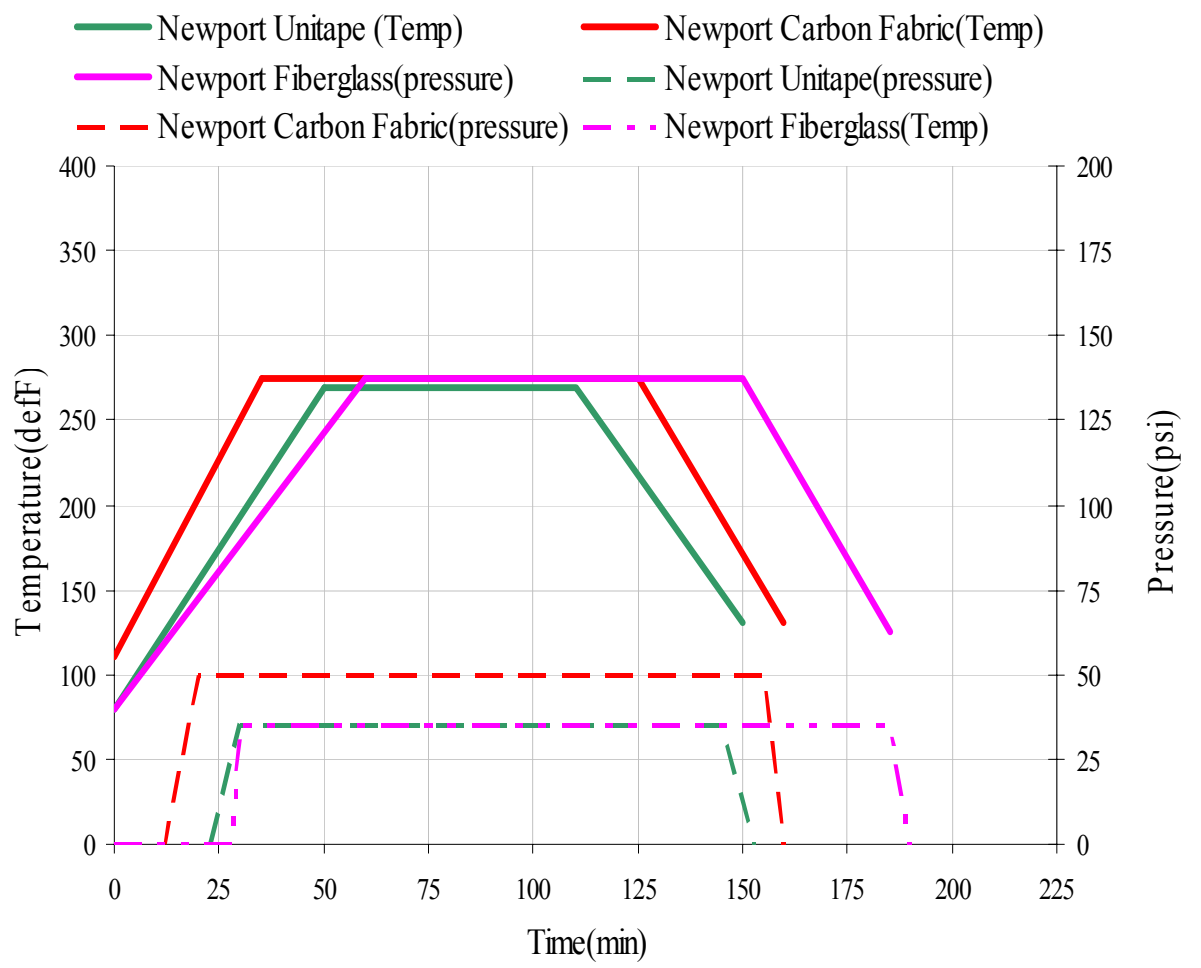

Figure 8. Cure cycle for Newport PWCF, Newport SWGF and Newport Unitape.

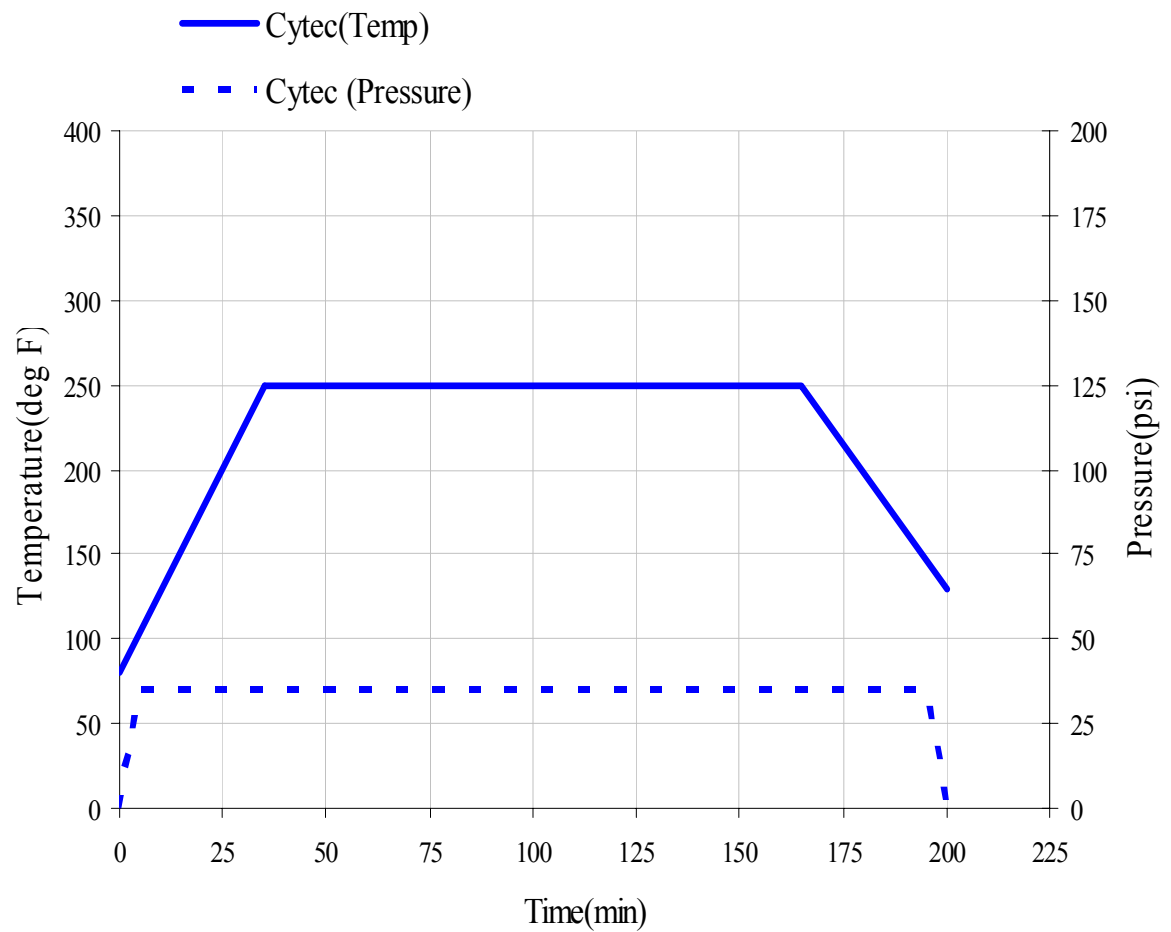

Figure 9.Cure Cycle for CytecPWC/T300/3KNT 


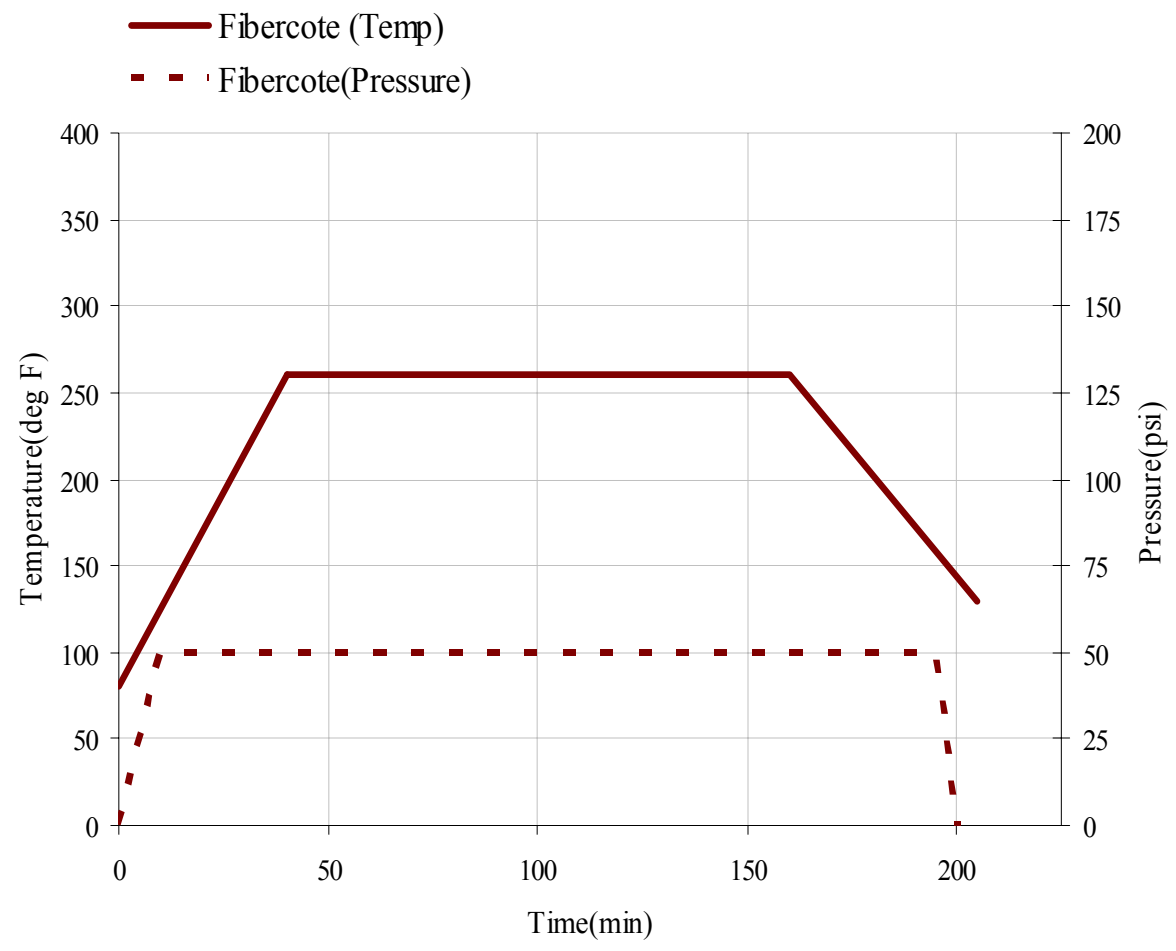

Figure 10. Cure Cycle for Fibercote 3KPW/E365

The shear testing was conducted at different stroke rates using high rate and 5 kip capacity servohydraulic machines. At each stroke rate, 3 coupons were tested. Table 1. Shows the test matrix for all the material systems used in the thesis work.

Table 1

Test Matrix

\begin{tabular}{|c|c|}
\hline $\begin{array}{c}\text { Constant Stroke Rate } \\
\text { in/sec }\end{array}$ & $\begin{array}{c}\text { No. of } \\
\text { Specimens }\end{array}$ \\
\hline 0.00083 & 3 \\
\hline 1 & 3 \\
\hline 10 & 3 \\
\hline 100 & 3 \\
\hline 250 & 3 \\
\hline 500 & 3 \\
\hline
\end{tabular}




\subsection{Specimen Preparation:}

Test specimens with notches were machined to size from the cured laminates per ASTM D7078-05. Cured Laminates are cut into rectangular specimen blanks by coolant lubricated precision cutting and grinding. The notches were cut in the specimen blanks using a diamond coated cutting tool. The specimen blanks were appropriately supported during the notching process to reduce the risk of delamination and matrix cracking. The typical test specimen is shown in Figure 11.

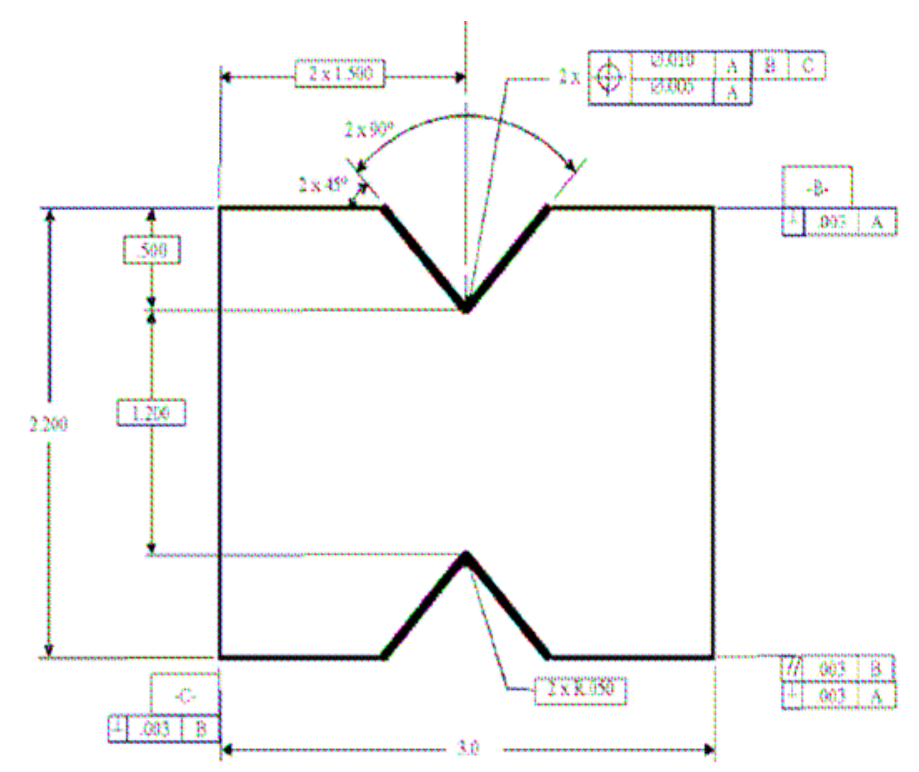

Figure 11.Test specimen geometry per ASTM D7078-05 [1].

\subsection{Labelling and Dimensioning:}

The specimens are labeled so that they will be distinct from each other and traceable back to the raw material and in a manner that will both be unaffected by the test and not influence the test. Dimensions are checked for all machined coupons and following were recorded; thickness, notch to notch width, length and width of the coupons using the appropriate measuring instruments. 


\section{CHAPTER 5}

\section{EXPERIMENTAL SETUP AND TESTING}

\subsection{Static Testing:}

Quasi-static shear testing was conducted on servo hydraulic MTS testing machine at constant actuator velocity of $0.05 \mathrm{in} / \mathrm{min}$. A 22kip MTS Load cell [19] was used for measuring the tensile load. All mechanical tests involved the measurement of displacements or strains, as defined by the ASTM D7078 standard. The pertinent dimensions of the test specimens were measured and recorded prior to bonding of strain gages to the specimens. Micro-Measurements EA125TH-120 [20], $\pm 45^{\circ}$ strain rosettes were bonded at the center of the gage section to measure the shear strains during the test. The strain gage is capable of measuring apparent maximum shear strain of 0.1 radians. To determine mechanical properties and ultimate load carrying capacity, three specimens of each material system were tested to failure at quasi-static stroke rate. All the materials specified in chapter 4. (Section 4.1) are tested at static rate to get the ultimate strength data at quasi-static stroke rate. The Figure 12 shows the stress-strain response for Cytec $\mathrm{PWC} / \mathrm{T} 300 / 3 \mathrm{KNT}$ material at $0.00083 \mathrm{in} / \mathrm{sec}$ stroke rate. The stress-strain curves are plotted for all material systems using the load and strain data recorded during the test by the MTS software. The stress-strain diagram is truncated at a shear strain value of 0.1 radians, which is the limit of the strain gage. The strain rate-strain diagram for Cytec PWC/T300/3KNT material at $0.00083 \mathrm{in} / \mathrm{sec}$ stroke rate is shown in the Figure 13 . In this graph we can clearly see that the strain rate is not constant for the total test time and is continuously changing. It can be concluded that the test was not conducted at constant strain rate. 


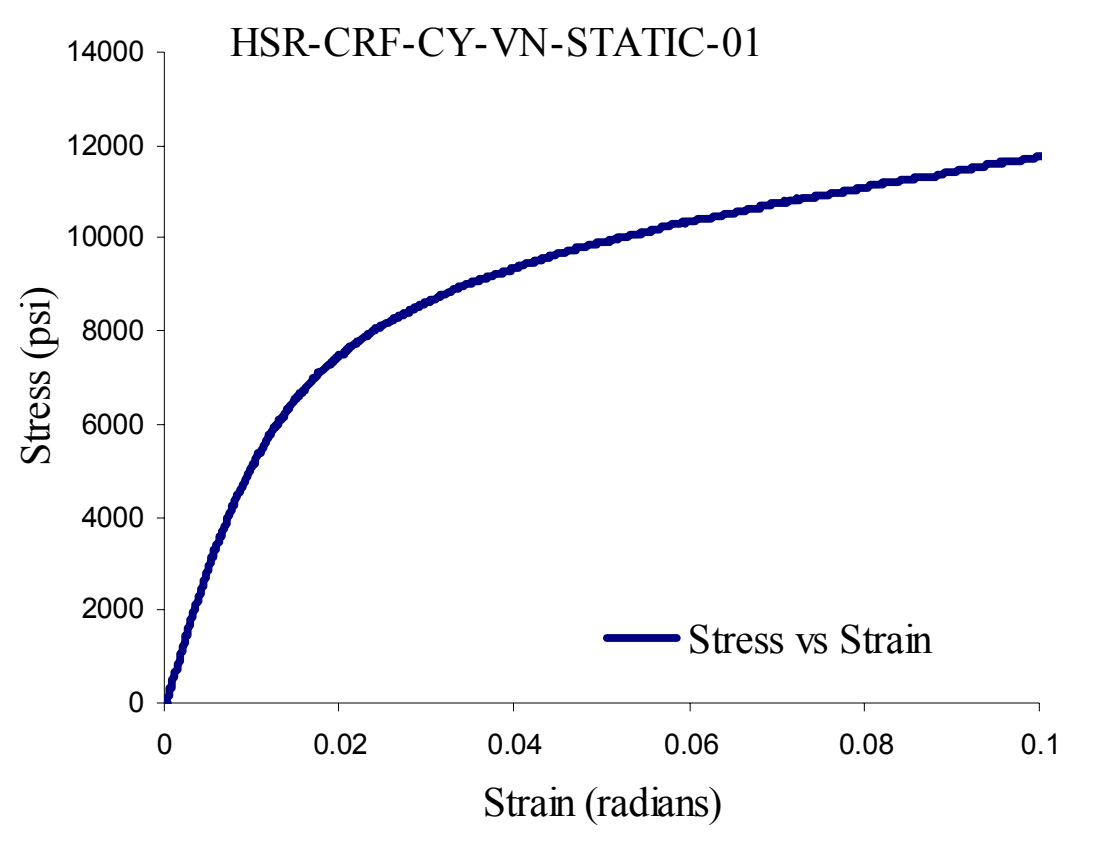

Figure 12. Stress-Strain response of Cytec PWC/T300/3KNT at $0.00083 \mathrm{in} / \mathrm{sec}$ stroke rate.

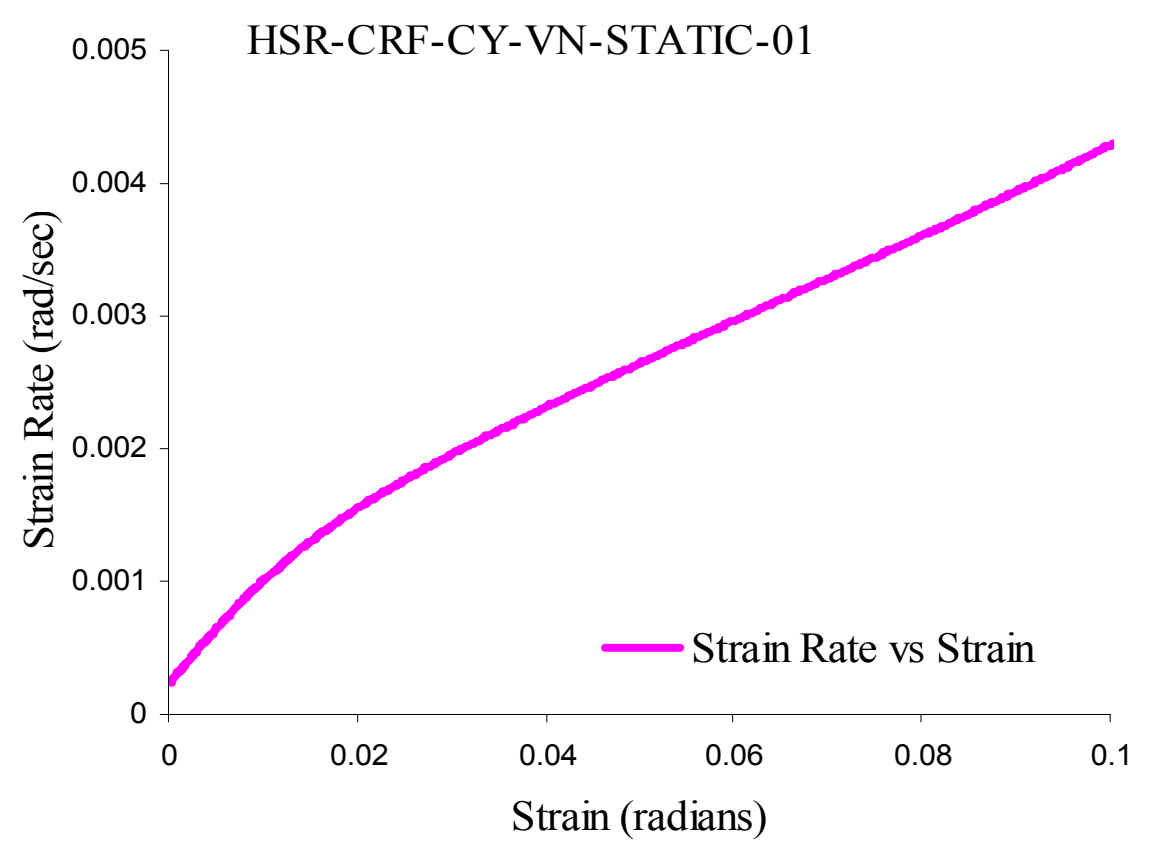

Figure 13. Strain Rate-Strain response of Cytec PWC/T300/3KNT at 0.00083 in/sec stroke rate. 


\subsection{High Rate Testing:}

The test specimens were tested at higher stroke rates using MTS 810 high rate servohydraulic testing machine. The testing machine is designed to have a stroke range of 15 inches and can reach speeds up to $500 \mathrm{in} / \mathrm{sec}$. The sustained loading capacity of the machine at rated speed is 5kips, while higher loads can be achieved at lower actuator speeds and for pulse loading.. The piston is accelerated to the required velocity before the grips are engaged to apply load to the specimen. Figure 14 shows the mechanical setup of the high rate tests. In this design, specimen is manually tightened in the top and bottom grips with high strength steel bolts. In between the bottom fixture and the hydraulic actuator shaft, a slack inducer mechanism is attached so that the desired speed can be achieved before the specimen starts taking actual load at that speed. A crushable foam ring is placed in between the bottom grip and the slack inducer to absorb the shock loads after specimens fail.

The supply pressure accumulators of the hydraulic actuator must be fully charged before the test as these provide the energy to achieve the "high speeds" which will be dissipated during the test. The machine was set up to ensure that there is enough slack to allow the actuator to reach its steady state velocity before engaging the specimen. Once engaged, the specimen is deformed until it fails. The load, displacement and strain are recorded by the high rate data acquisition system (National Instruments data acquisition system). The main experimental feature of this investigation is to use exactly the same coupon configuration and strain gages both for quasi-static and dynamic tests. Typical signals from the compact-size piezoelectric PCB PIEZOTRONICS $^{\mathrm{INC}}$ [21] load cell are obtained for getting the load values in terms of voltages and then the calibration sheet provided by the manufacturer is used to convert the load from voltage to pounds. 


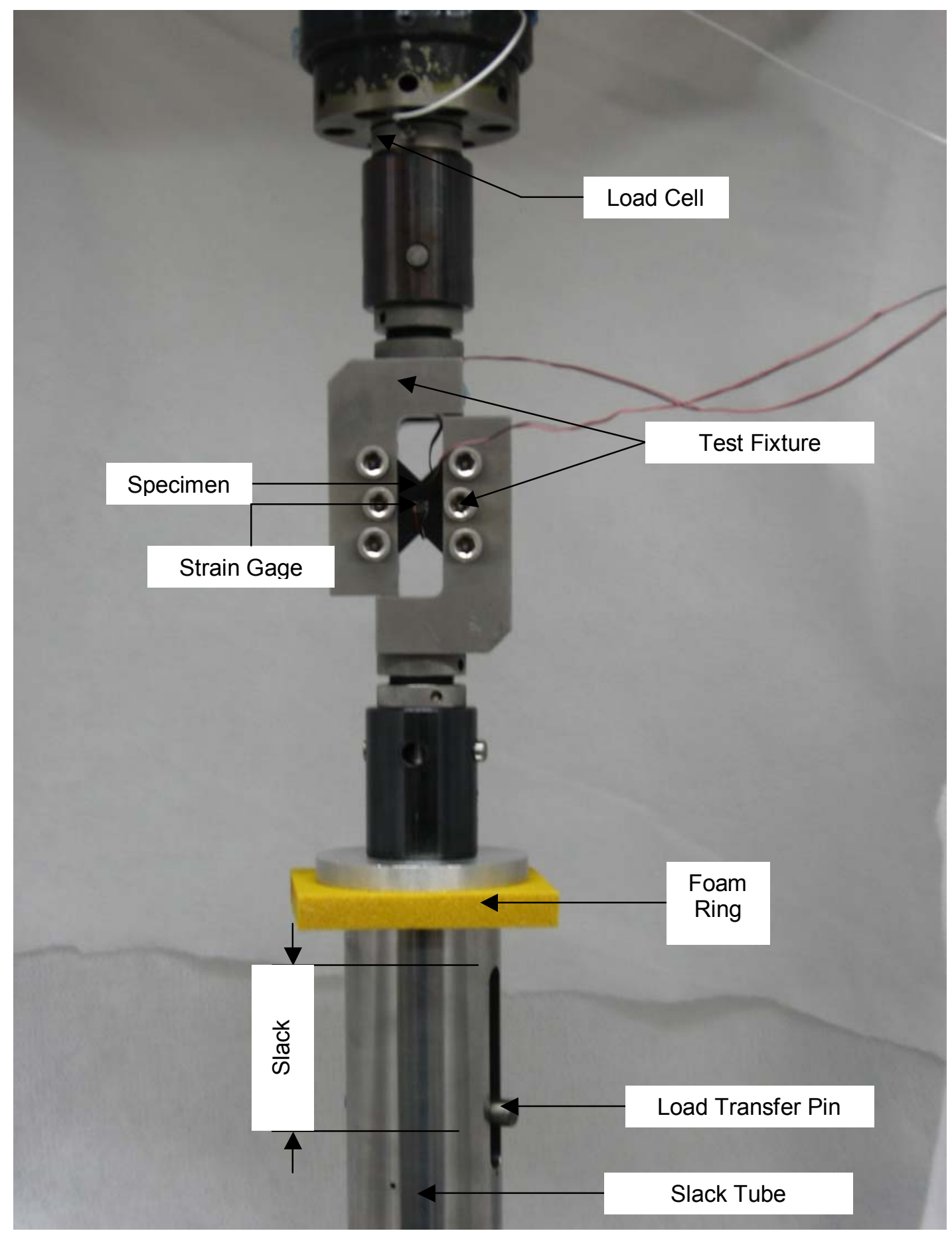

Figure 14. Setup for High Rate Testing

The load cell is calibrated in compression mode and with a customer supplied bolt as per the requirements of mechanical fittings available on the test fixture. Load cell is preloaded to 
$26000 \mathrm{lbs}$ prior to calibration. The Vishay 2210 signal conditioning amplifier system is used to condition strain gage signals.

\subsection{Description of Test Data Reduction:}

The time histories of shear strain and stress (or load) for a test conducted at $250 \mathrm{in} / \mathrm{sec}$ is illustrated in the Figure 15. During the test the strain measurement occurs instantaneously and it leads the load signal in time, especially for the tests conducted at $100 \mathrm{in} / \mathrm{sec}$ or higher. The load signal is modulated in phase and amplitude due to the load path in between the test fixture and the load cell. The load signal modulation is also affected by the mass and stiffness of the test fixture. The phase difference between the strain and load signals will be increases with stroke rate. For the experimental set-up used in the current study the phase difference is negligible for stroke rates less than $10 \mathrm{in} / \mathrm{sec}$. In order to plot the stress-strain curves, the load (or strain) signal was shifted in time. The load signal must be further corrected for amplitude modulation using system transfer function, which is part of ongoing work. The results however do not incorporate any corrections to the load signal amplitude. 


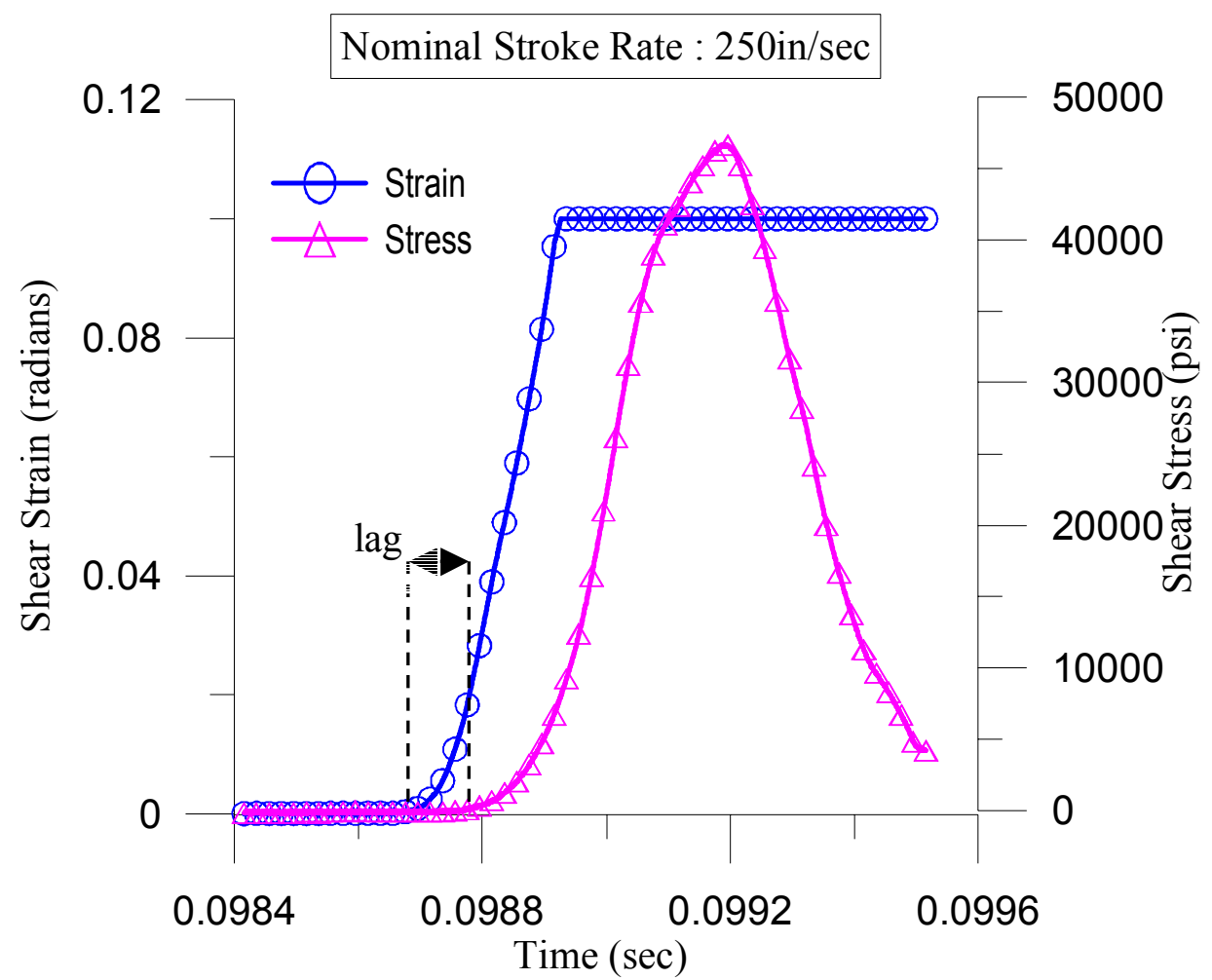

Figure 15. Time lag between strain and stress signals during a high-rate test.

In this present study, to obtain the strain rate throughout the test, strain-time curves are plotted for each stroke rate test as shown in the Figure 16. This strain-time data when differentiated with respect to time provides the strain rate. The red line is the best fit polynomial for the strain-time curve for a $250 \mathrm{in} / \mathrm{s}$ stroke rate test of Cytec PWC/T300/3KNT material. The strain data obtained from the test contains noisy data points which are inherent to high speed testing. So, a best fit polynomial is used to smooth those noisy data points. The time averaged value of strain rate is used to associate the test data with a nominal "average strain rate". 


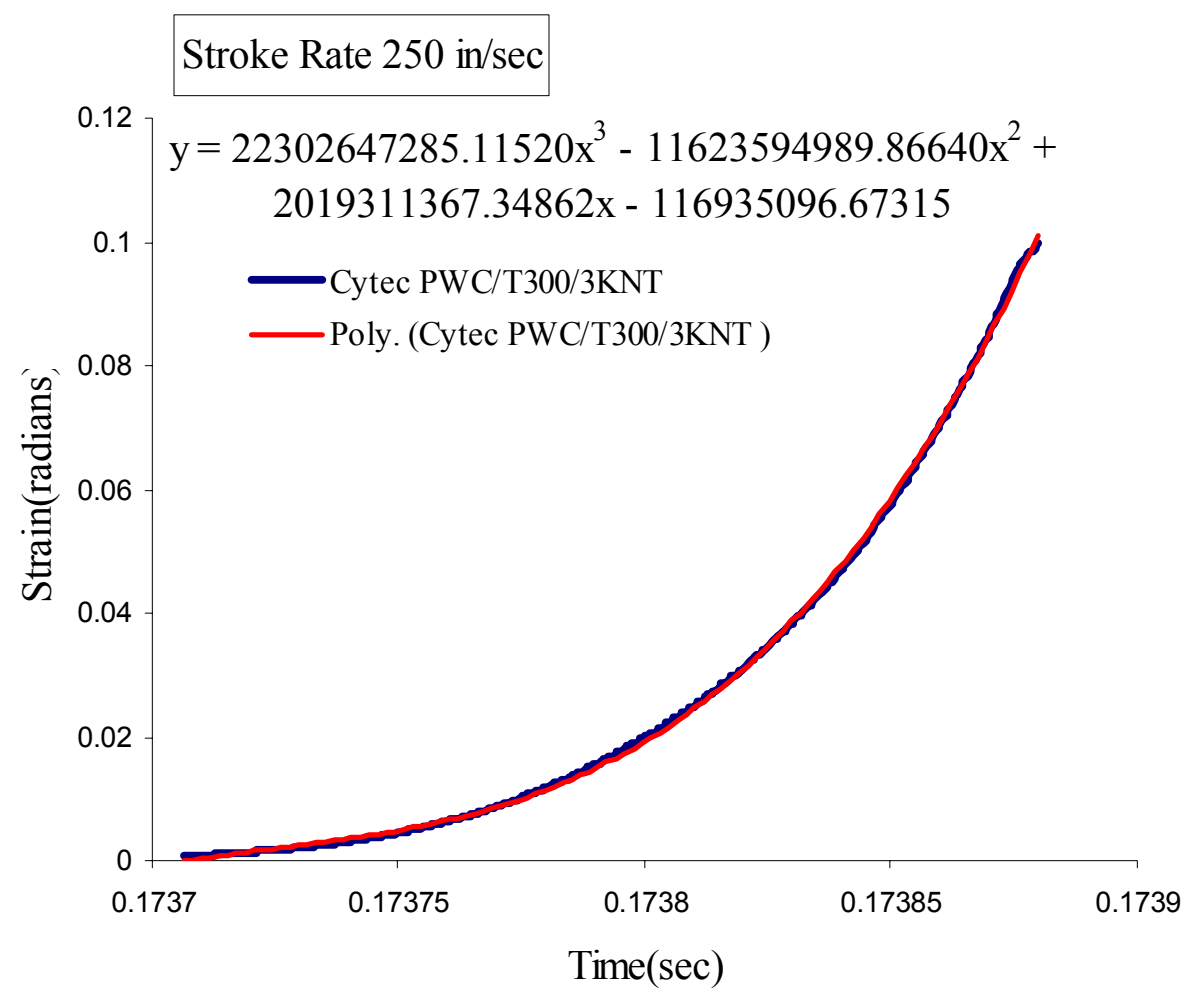

Figure 16. Strain-Time data for Cytec PWC/T300/3KNT at 250in/sec stroke rate. 


\section{CHAPTER 6}

\section{EXPERIMENTAL RESULTS AND DISCUSSION}

\subsection{Stress-Strain Response at Quasi-Static Loading:}

The shear stress versus actuator displacement and shear stress-strain plots were obtained from tests conducted at quasi-static rates are illustrated in Figures 17 and 18 for all the material systems investigated. All the materials exhibit identical behavior as seen from the Figure 17. The stress-strain diagram are truncated at a shear strain value of 0.1 radians, which is the physical limit of the strain gage.

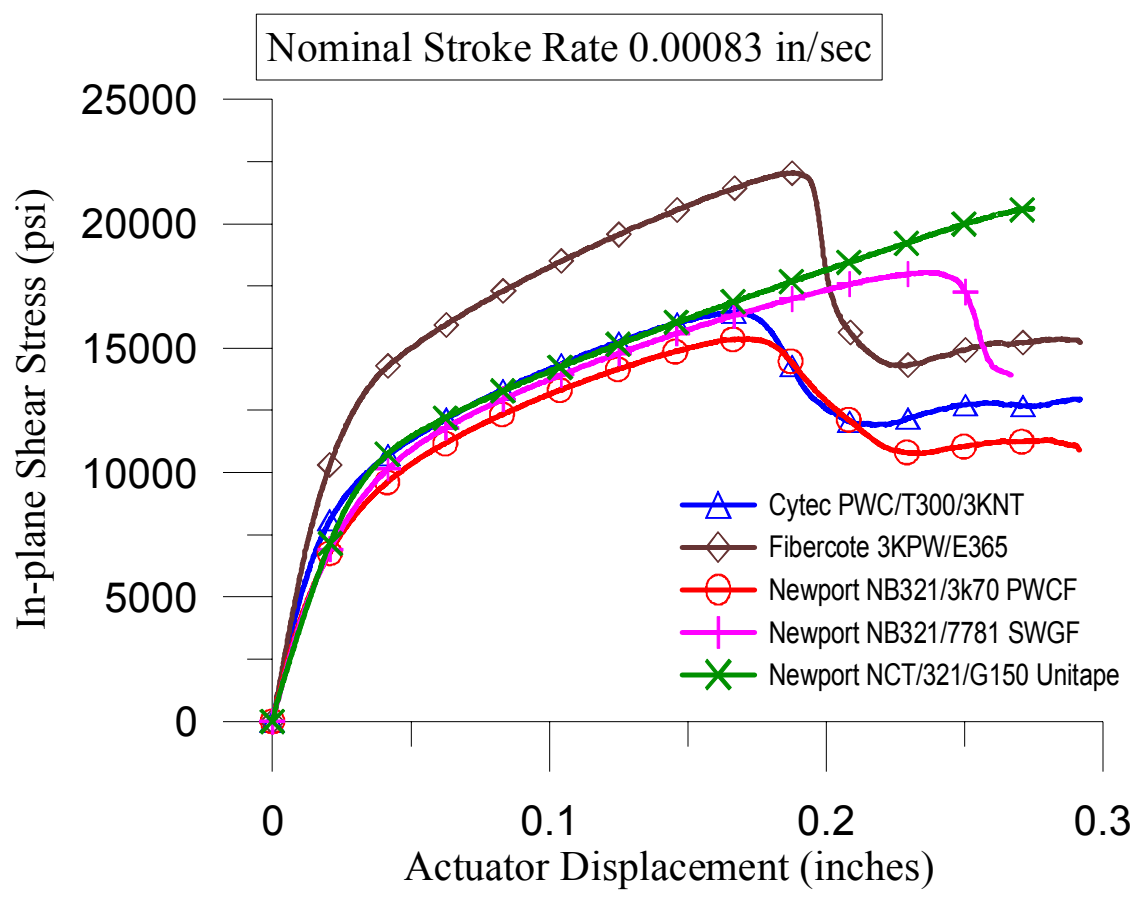

Figure 17. Shear stress-Displacement diagram for the materials tested at quasi static test rate. 


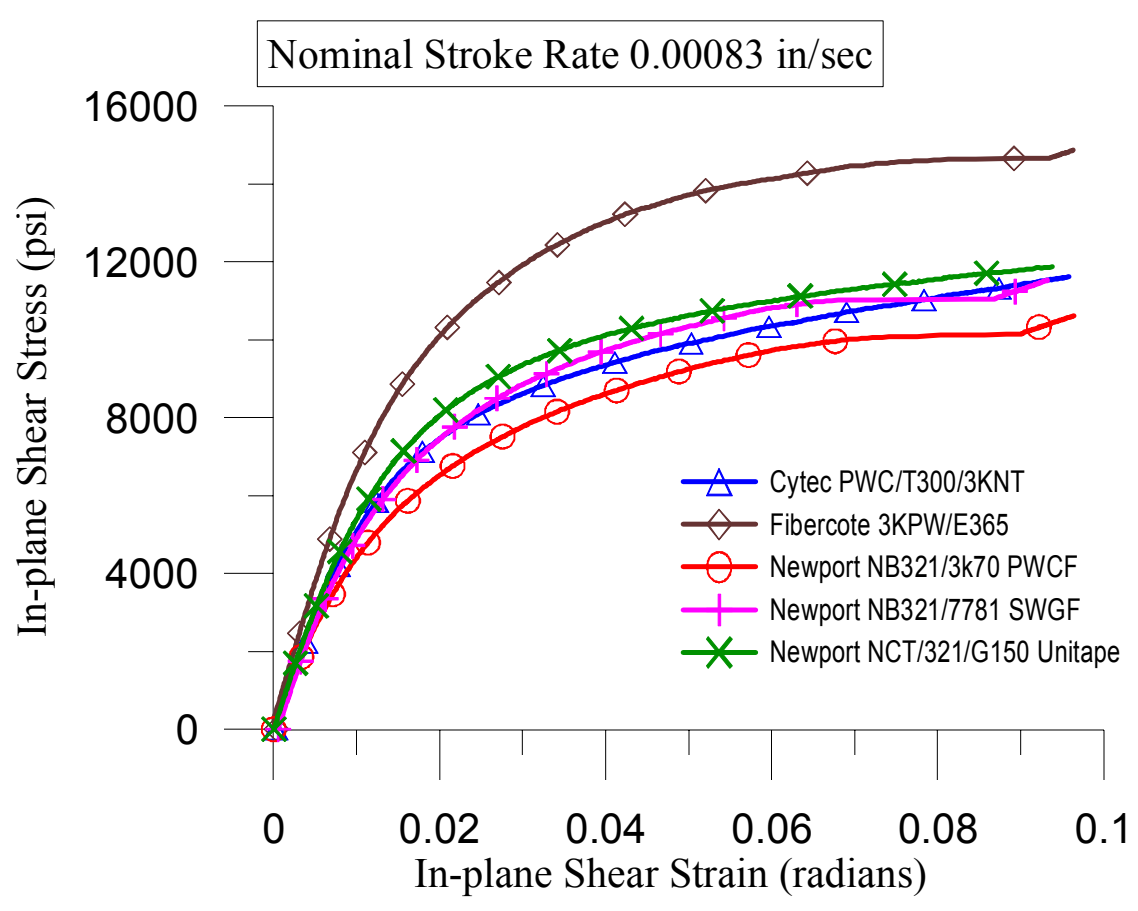

Figure 18. Shear stress-shear strain diagram for the materials tested at quasi static test rate.

The shear stress-strain behavior of all the materials recorded at tests conducted at different stroke rates is illustrated in the figures 19, 20, 21, 22 and 23. The shapes of the stressstrain curves are similar to that of the quasi-static test, up to a stroke rate of $100 \mathrm{in} / \mathrm{sec}$, for all the materials. At any strain level, the shear stress level increase with stroke rate for Newport materials. For Fibercote 3KPW/E365 and Cytec PWC/T300/3KNT materials shear stress level falls beyond $100 \mathrm{in} / \mathrm{sec}$ stroke rate. At stroke rates of $100 \mathrm{in} / \mathrm{sec}$ and higher, the stress-strain behavior appears to change significantly, with an appreciable decrease in material stiffness. 


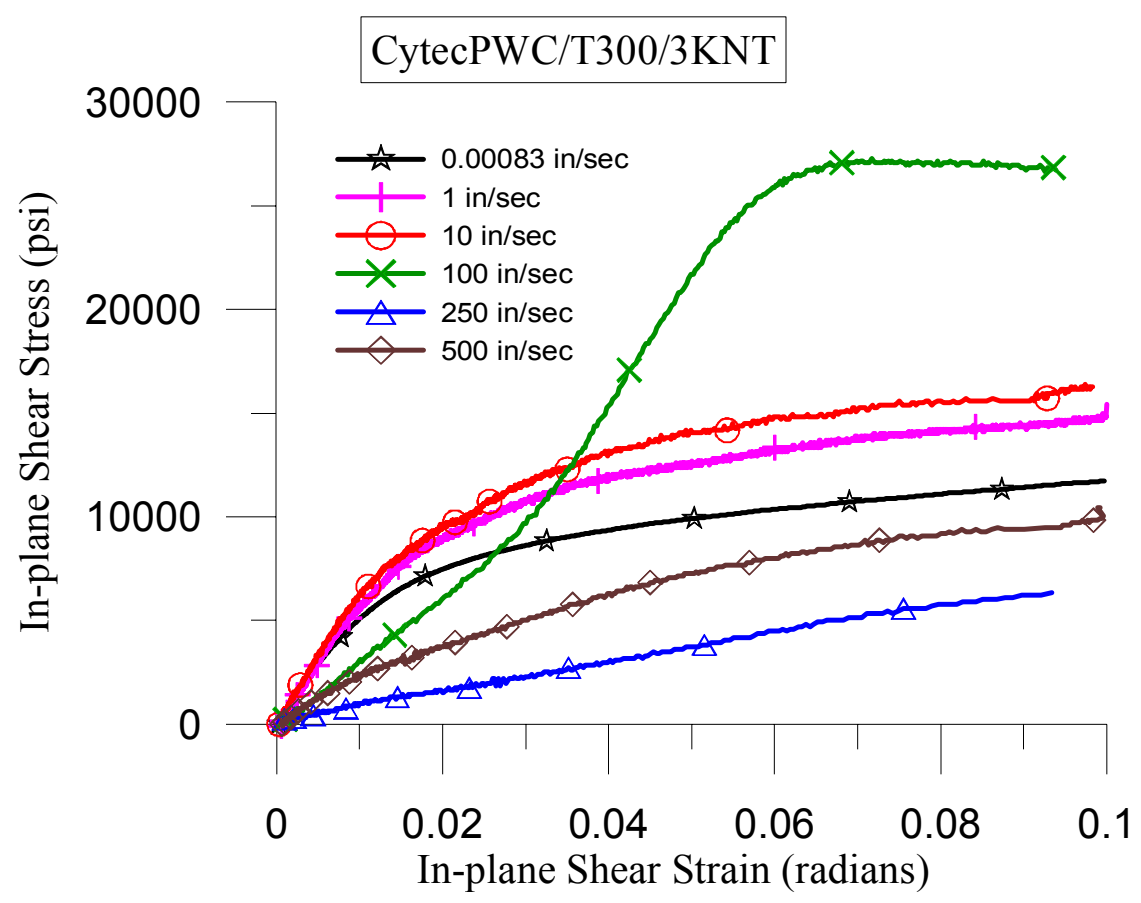

Figure 19. In-plane shear stress-shear strain behavior of Cytec PWC/T300/3KNT material at different stroke rates.

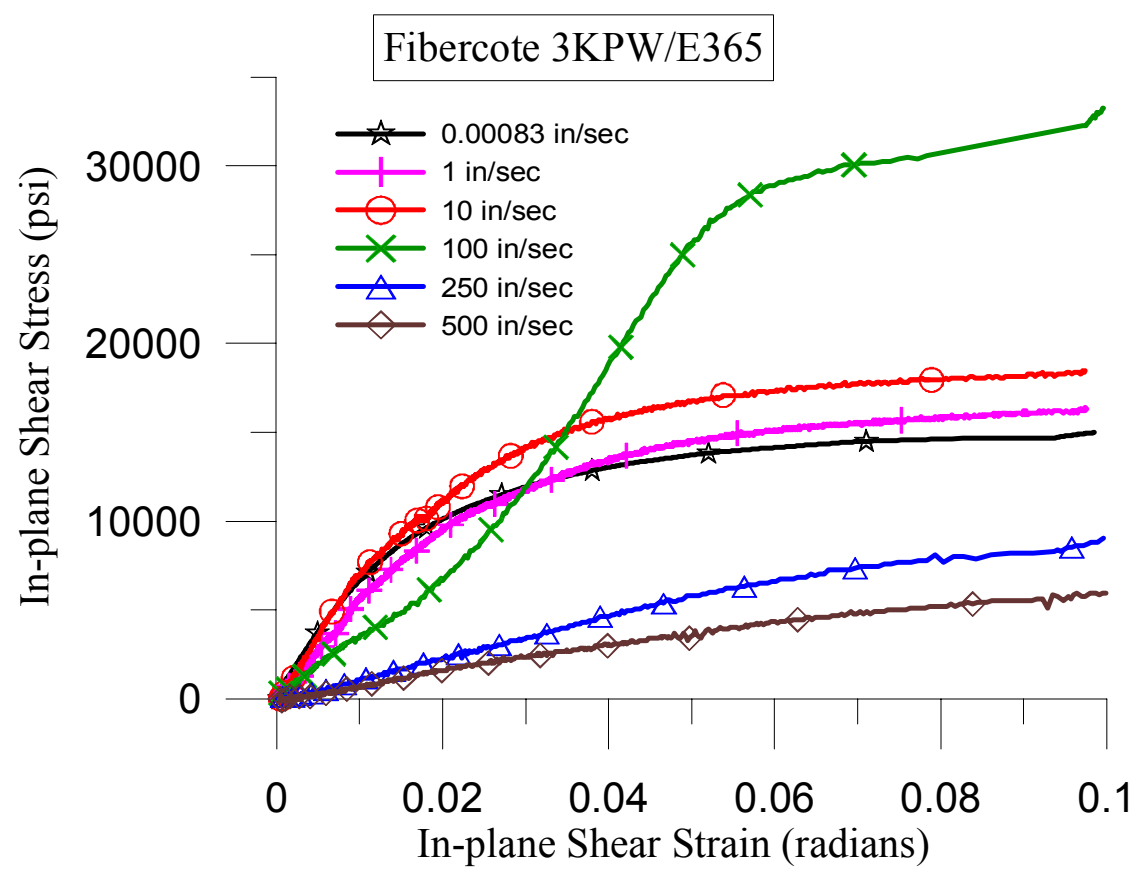

Figure 20. In-plane shear stress-shear strain behavior of Fibercote 3KPW/E365 material at different stroke rates. 


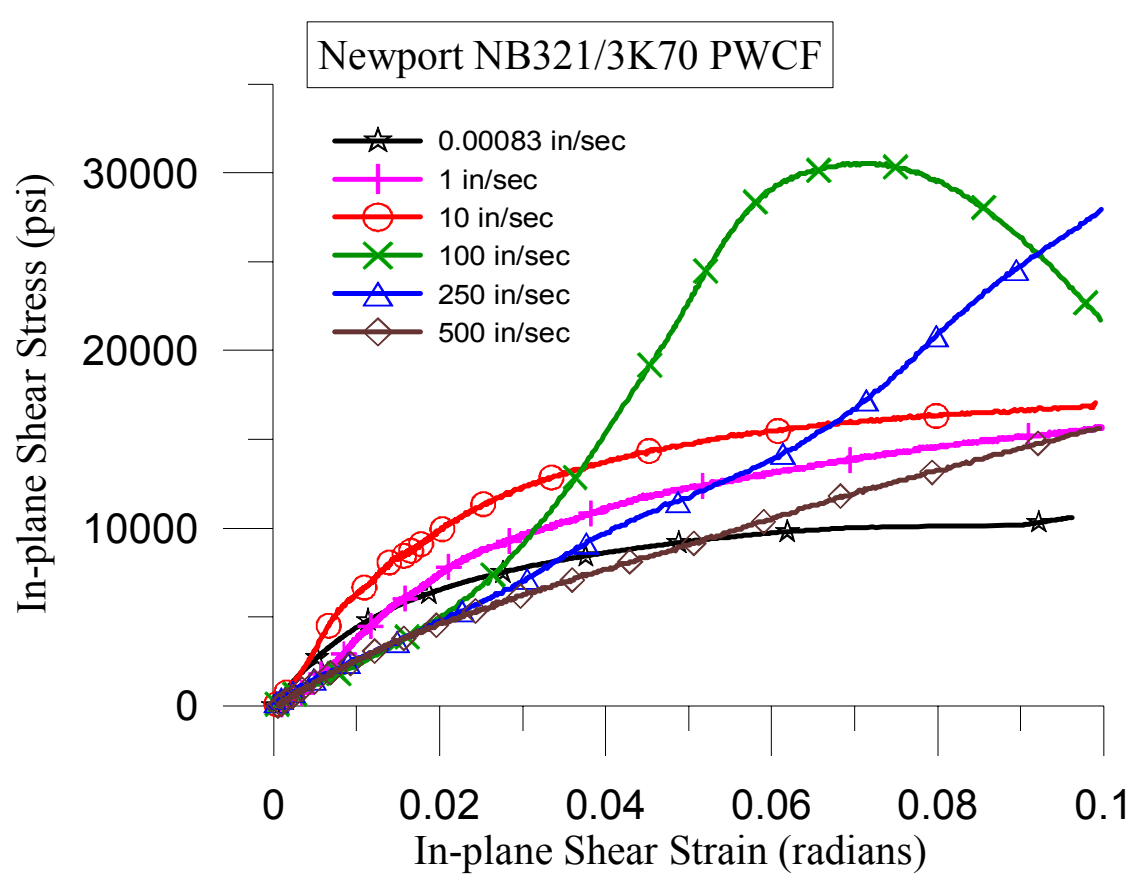

Figure 21. In-plane shear stress-shear strain behavior of Newport PWCF material at different stroke rates.

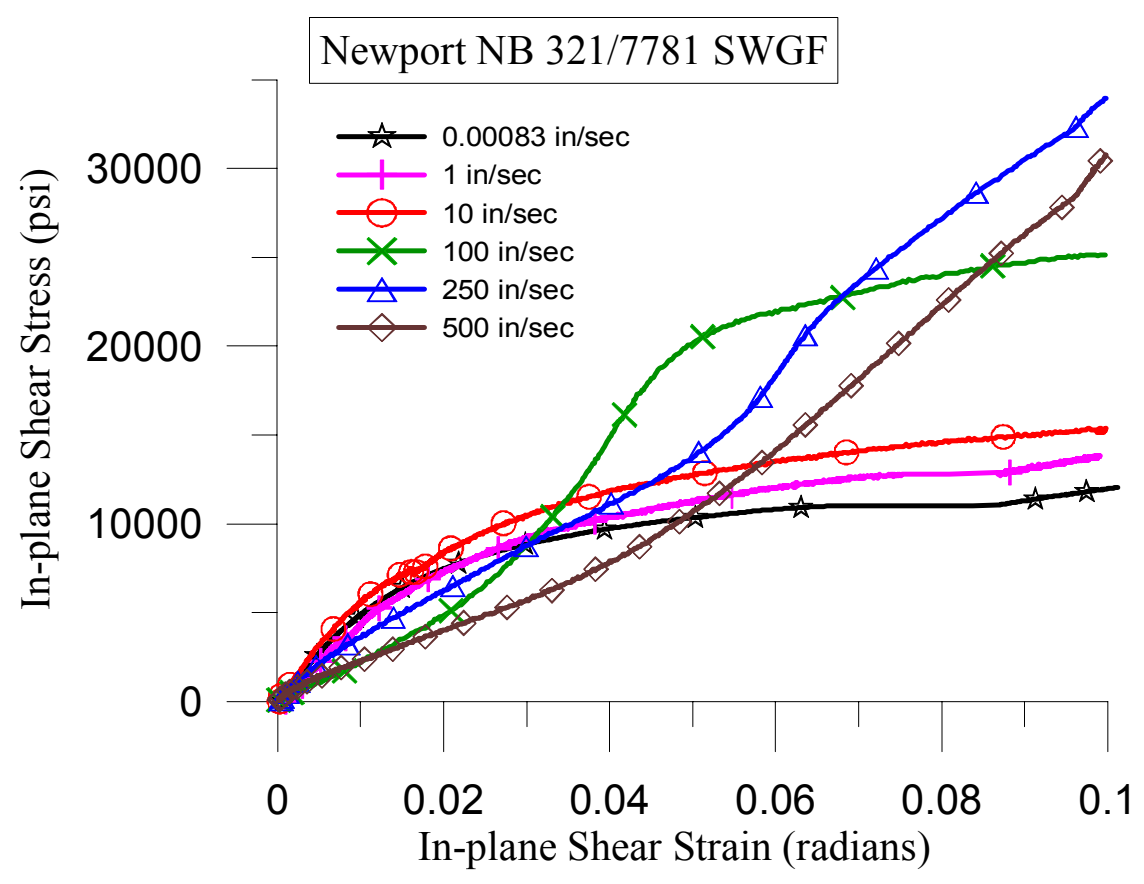

Figure 22. In-plane shear stress-shear strain behavior of Newport SWGF material at different stroke rates. 


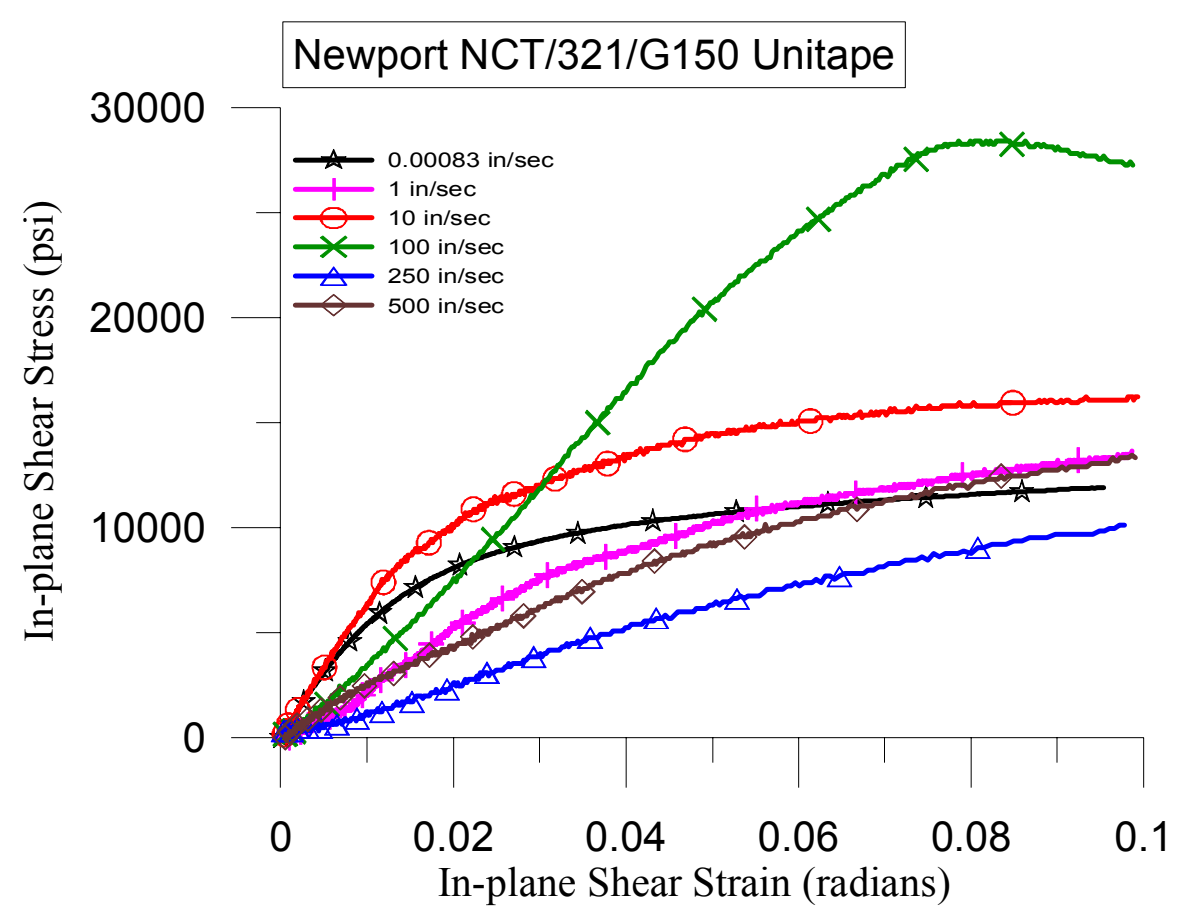

Figure 23. In-plane shear stress-shear strain behavior of Newport Unitape material at different stroke rates.

The summary of the test data for all the material systems are shown in tables 2,3,4,5 and 6 .

Table 2

Summary of test data for Cytec PWC/T300/3KNT material

\begin{tabular}{|c|c|c|c|}
\hline Orientation & $\begin{array}{c}\text { Nominal Stroke Rate } \\
(\mathrm{in} / \mathrm{s})\end{array}$ & $\begin{array}{c}\text { Ultimate Failure Stress } \\
\left(\mathrm{lbf} / \mathrm{in}^{2}\right)\end{array}$ & $\begin{array}{c}\text { Average Strain Rate } \\
(\mathrm{rad} / \mathrm{sec})\end{array}$ \\
\hline $0^{\circ}$ & 0.00083 & 16448.10 & 0.0024 \\
\hline $0^{\circ}$ & 1 & 18779.24 & 0.98 \\
\hline $0^{\circ}$ & 10 & 21026.05 & 8.25 \\
\hline $0^{\circ}$ & 100 & 33406.73 & 236.94 \\
\hline $0^{\circ}$ & 250 & 23462.18 & 539.09 \\
\hline $0^{\circ}$ & 500 & 24431.50 & 588.83 \\
\hline
\end{tabular}


Table 3

Summary of test data for Fibercote 3KPW/E365 material

\begin{tabular}{|c|c|c|c|}
\hline Orientation & $\begin{array}{c}\text { Nominal Stroke Rate } \\
(\mathrm{in} / \mathrm{s})\end{array}$ & $\begin{array}{c}\text { Ultimate Failure Stress } \\
\left(\mathrm{lbf} / \mathrm{in}^{2}\right)\end{array}$ & $\begin{array}{c}\text { Average Strain Rate } \\
(\mathrm{rad} / \mathrm{sec})\end{array}$ \\
\hline $0^{\circ}$ & 0.00083 & 22062.18 & 0.0007 \\
\hline $0^{\circ}$ & 1 & 22837.93 & 1.01 \\
\hline $0^{\circ}$ & 10 & 24843.64 & 7.53 \\
\hline $0^{\circ}$ & 100 & 39993.56 & 254.89 \\
\hline $0^{\circ}$ & 250 & 42662.26 & 551.54 \\
\hline $0^{\circ}$ & 500 & 29151.74 & 654.81 \\
\hline
\end{tabular}

Table 4

Summary of Test Data for Newport PWCF Material

\begin{tabular}{|c|c|c|c|}
\hline Orientation & $\begin{array}{c}\text { Nominal Stroke Rate } \\
(\mathrm{in} / \mathrm{s})\end{array}$ & $\begin{array}{c}\text { Ultimate Failure Stress } \\
\left(\mathrm{lbf} / \mathrm{in}^{2}\right)\end{array}$ & $\begin{array}{c}\text { Average Strain Rate } \\
(\mathrm{rad} / \mathrm{sec})\end{array}$ \\
\hline $0^{\circ}$ & 0.00083 & 17374.84 & 0.0016 \\
\hline $0^{\circ}$ & 1 & 21540.40 & 1.03 \\
\hline $0^{\circ}$ & 10 & 20715.40 & 8.26 \\
\hline $0^{\circ}$ & 100 & 30670.91 & 172.66 \\
\hline $0^{\circ}$ & 250 & 42399.18 & 380.32 \\
\hline $0^{\circ}$ & 500 & 45828.37 & 479.94 \\
\hline
\end{tabular}


Table 5

Summary of Test Data for Newport SWGF Material

\begin{tabular}{|c|c|c|c|}
\hline Orientation & $\begin{array}{c}\text { Nominal Stroke Rate } \\
(\mathrm{in} / \mathrm{s})\end{array}$ & $\begin{array}{c}\text { Ultimate Failure Stress } \\
\left(\mathrm{lbf} / \mathrm{in}^{2}\right)\end{array}$ & $\begin{array}{c}\text { Average Strain Rate } \\
(\mathrm{rad} / \mathrm{sec})\end{array}$ \\
\hline $0^{\circ}$ & 0.00083 & 18057.65 & 0.0005 \\
\hline $0^{\circ}$ & 1 & 19857.77 & 1.13 \\
\hline $0^{\circ}$ & 10 & 22329.94 & 9.85 \\
\hline $0^{\circ}$ & 100 & 28658.64 & 225.76 \\
\hline $0^{\circ}$ & 250 & 42848.20 & 335.30 \\
\hline $0^{\circ}$ & 500 & 46621.61 & 374.71 \\
\hline
\end{tabular}

Table 6

Summary of Test Data for Newport Unitape Material

\begin{tabular}{|c|c|c|c|}
\hline Orientation & $\begin{array}{c}\text { Nominal Stroke Rate } \\
(\mathrm{in} / \mathrm{s})\end{array}$ & $\begin{array}{c}\text { Ultimate Failure Stress } \\
\left(\mathrm{lbf} / \mathrm{in}^{2}\right)\end{array}$ & $\begin{array}{c}\text { Average Strain Rate } \\
(\mathrm{rad} / \mathrm{sec})\end{array}$ \\
\hline $0^{\circ}$ & 0.00083 & 17946.14 & 0.0015 \\
\hline $0^{\circ}$ & 1 & 23814.61 & 1.24 \\
\hline $0^{\circ}$ & 10 & 24329.16 & 9.30 \\
\hline $0^{\circ}$ & 100 & 40339.12 & 204.62 \\
\hline $0^{\circ}$ & 250 & 24389.94 & 538.42 \\
\hline $0^{\circ}$ & 500 & 25471.76 & 621.82 \\
\hline
\end{tabular}


From the test data presented it is observed that the failure stress is changing with respect to the stroke rate. The average strain rate mentioned in the tables the mean value taken for the strain rate values recorded at specific equal time intervals. From the data presented above we can observe that the material strength is increasing with respect to the strain rate. The stress-strain behavior of all the materials at stroke rates of 100, 250 and 500in/sec are compared in figures 24 , 25 and 26. It can be observed that the stress-strain behaviors are similar up to a shear strain level of 0.04 radians for the Newport materials but Cytec PWC/T300/3KNT, Fibercote 3KPW/E365 and Newport unitape materials behave in a contrasting manner depending on the test speed. At a test speed of $100 \mathrm{in} / \mathrm{sec}$, the Newport fiberglass material reaches a plateau earlier than the other materials, but at higher rates, the Newport NB321/7781 fiberglass material exhibits additional stiffening compared to other materials. In materials Cytec PWC/T300/3KNT, Fibercote 3KPW/E365 and Newport unitape there was a gradual decrease in the stiffness with the stroke rate.

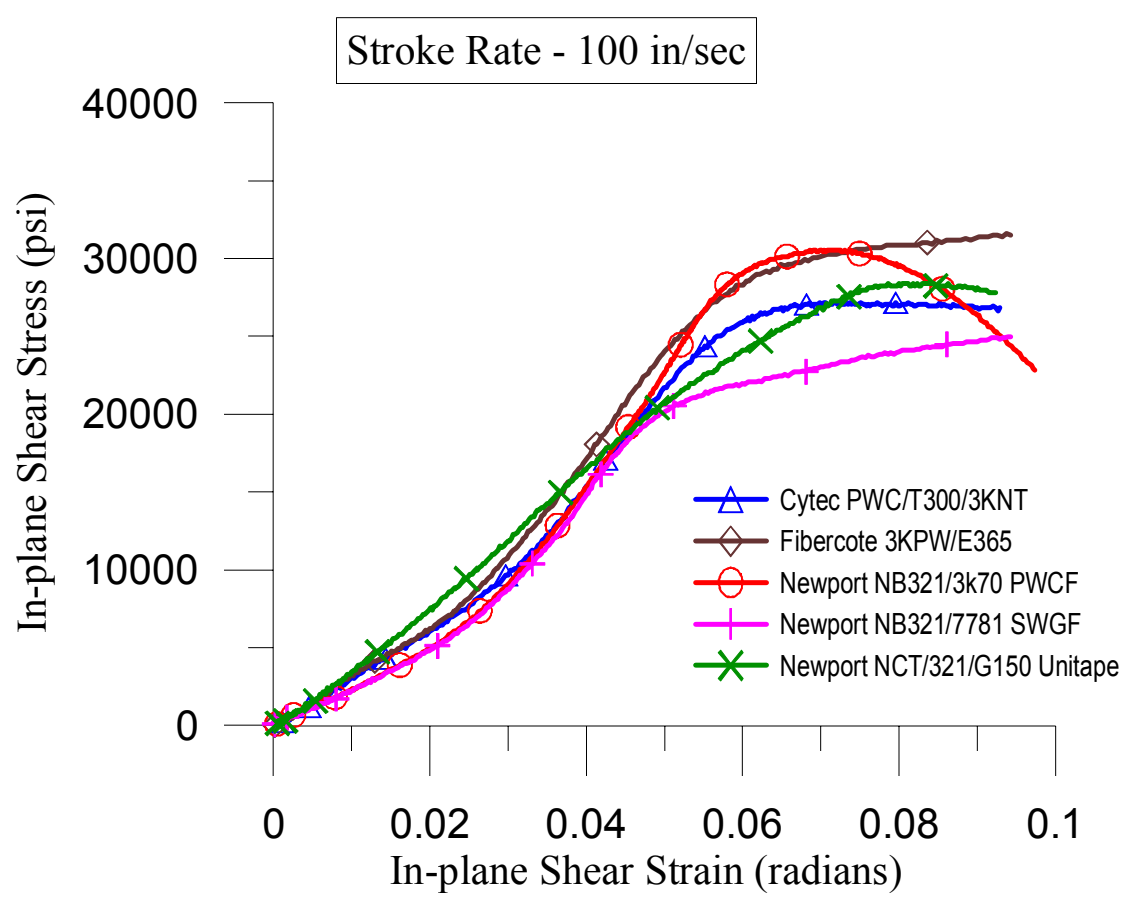

Figure 24. In-plane shear stress- strain at 100in/sec 


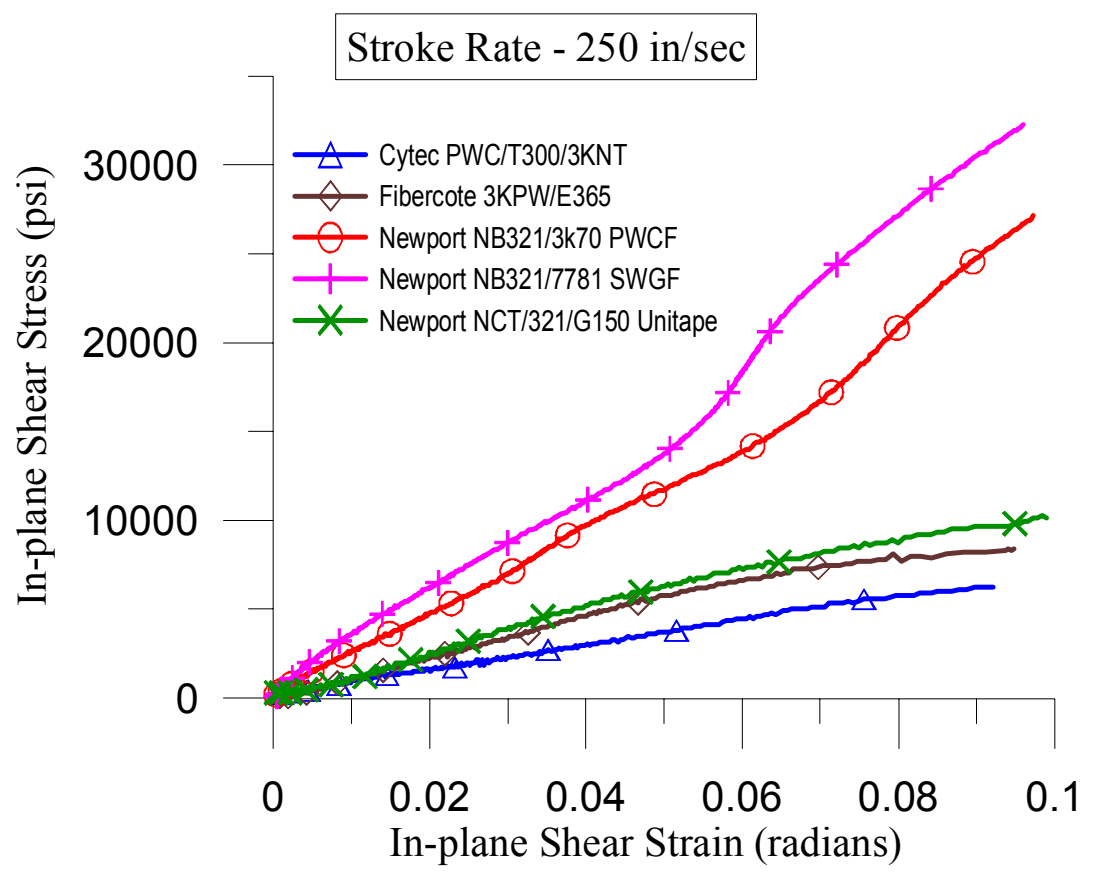

Figure 25. In-plane shear stress- strain at $250 \mathrm{in} / \mathrm{sec}$

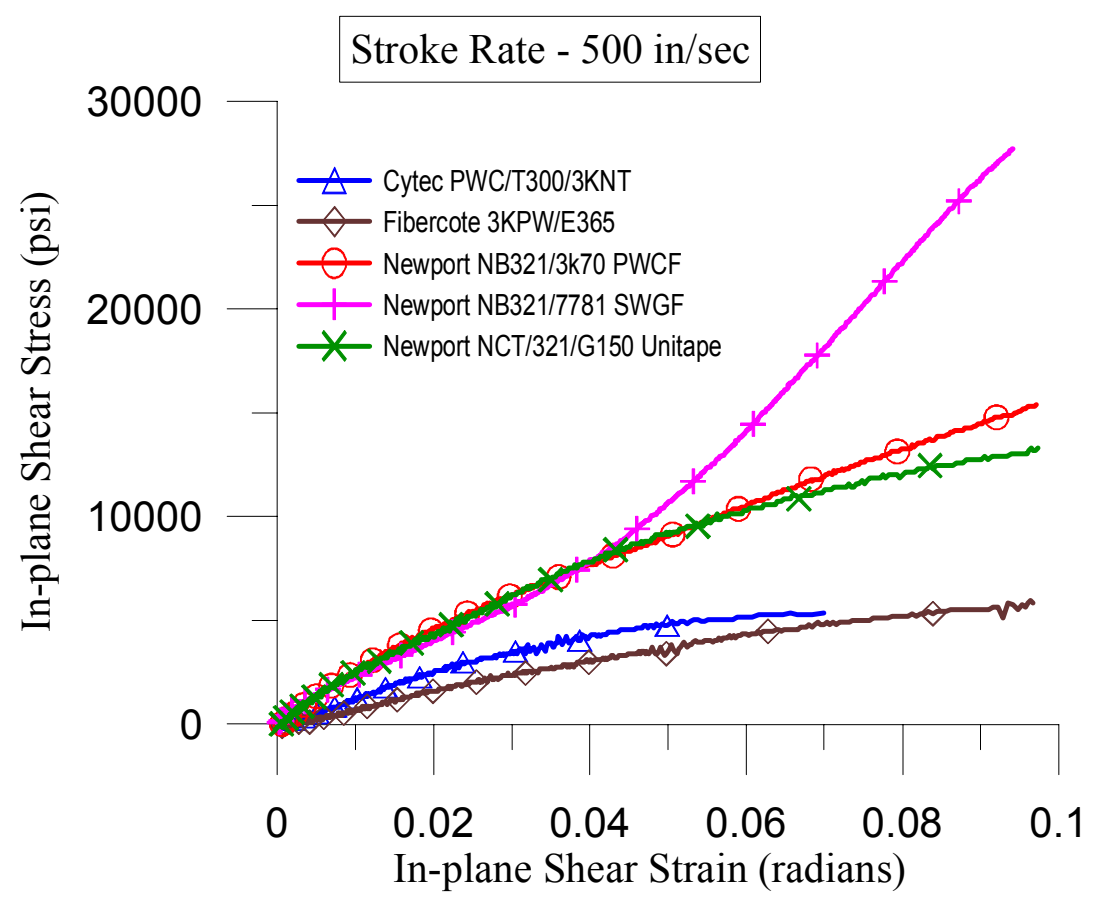

Figure 26. In-plane shear stress- strain at $500 \mathrm{in} / \mathrm{sec}$ 


\subsection{Strength of Material Systems:}

The in-plane shear strength of the materials were plotted as a function of nominal stroke rate in Figure 27. The in-plane shear strengths increase with stroke rate, with differences existing between the material systems. There was not much increase in the strengths for Newport Unitape, Cytec PWC/T300/3KNT and Fibercote 3KPW/E365 materials which was seen in the other Newport material systems. From Figure 28, it can be observed that average strain rate increases with stroke rate for all materials tested.

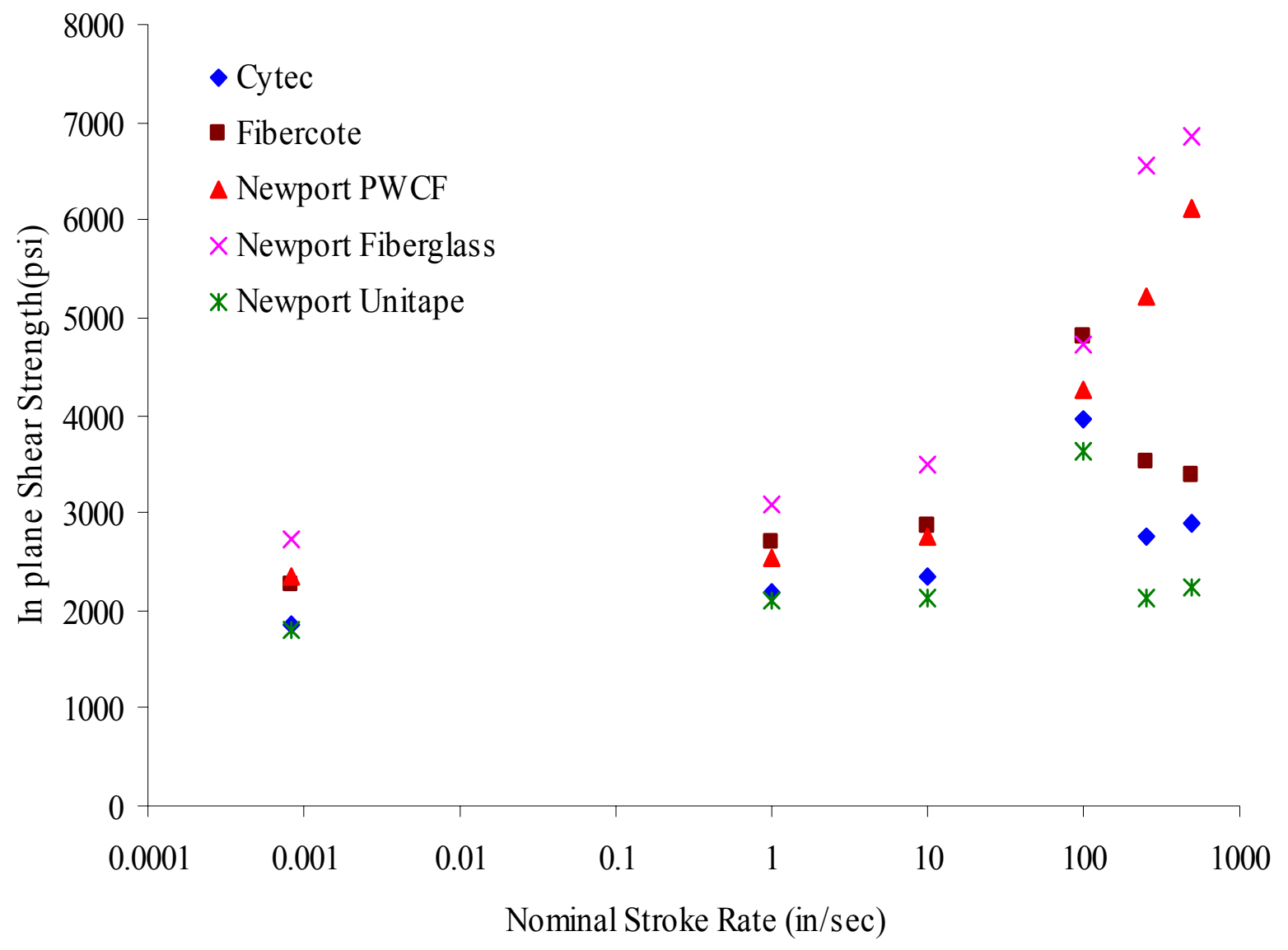

Figure 27. Strength of the material systems at different stroke rates. 


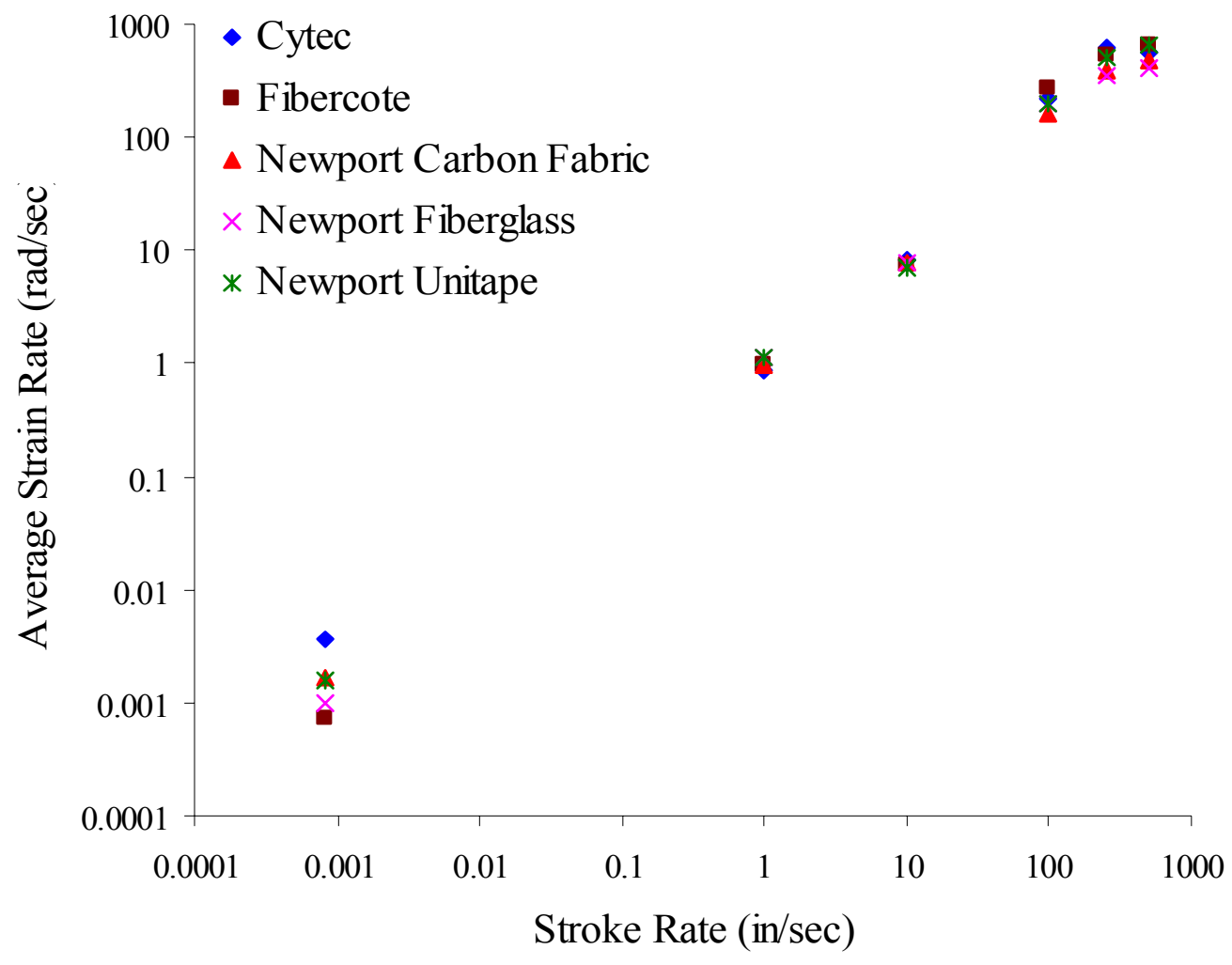

Figure 28. Average Strain Rates of material systems at different stroke rates.

\subsection{Failure modes:}

The failure modes in the test specimens were examined post-test to investigate the influence of strain rate. The failure modes in Cytec PWC/T300/3KNT, Fibercote 3KPW/E365, Newport Unitape specimens tested at different rates and Newport PWCF specimens tested at rates up to 100in/sec was primarily shear failure across the minimum cross-section. The shear failure occurs over a narrow band between the notches. The failure surface tends to be oriented at a shallow angle to the plane of the specimen, with fractured fibers protruding out of the surface. However, at higher rates for Newport PWCF and Newport SWGF specimens fail across sections that are not necessarily the minimum, as illustrated in the Figure 32 and 33. Further, the fracture surface tends to be perpendicular to the plane of the specimen with relatively less fibers protruding 
across the fracture surface, when compared to that of quasi-static failure modes. For Newport SWGF a large band of process zone was visible due to the translucency of the material which can be seen clearly in the Figure 33. The width of this band was observed to increase with loading rate. For materials Cytec PWC/T300/3KNT, Fibercote 3KPW/E365 and Newport Unitape the failure modes were seen across the minimum section of the coupons for all the test rates which is shown in Figure 29, 30 and 31.

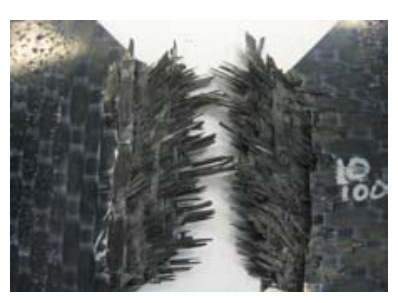

(a) $100 \mathrm{in} / \mathrm{sec}$

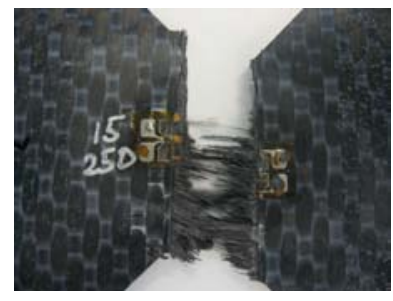

(b) $250 \mathrm{in} / \mathrm{sec}$

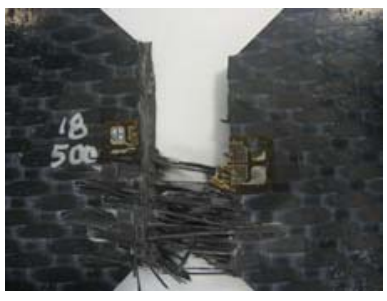

(c) $500 \mathrm{in} / \mathrm{sec}$

Figure 29. Failure modes in Cytec PWC/T300/3KNT material at different stroke rates

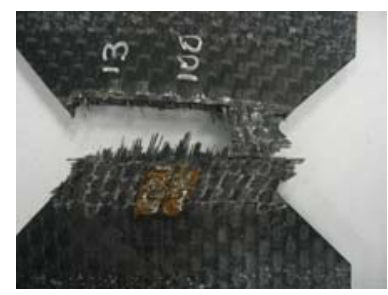

(a) $100 \mathrm{in} / \mathrm{sec}$

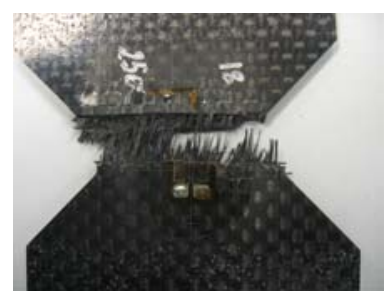

(b) $250 \mathrm{in} / \mathrm{sec}$

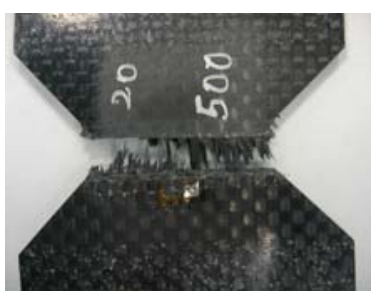

(c) $500 \mathrm{in} / \mathrm{sec}$

Figure 30. Failure modes in Fibercote 3KPW/E365 material at different stroke rates

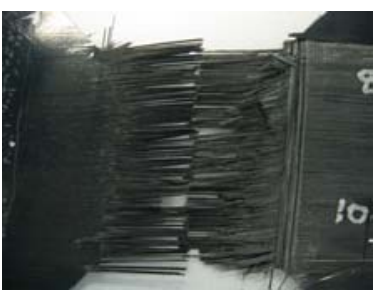

(a) $100 \mathrm{in} / \mathrm{sec}$

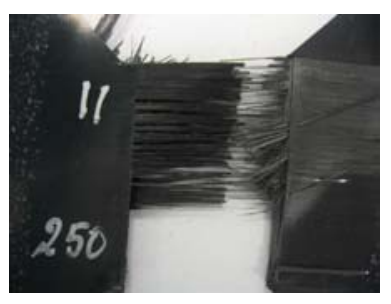

(b) $250 \mathrm{in} / \mathrm{sec}$

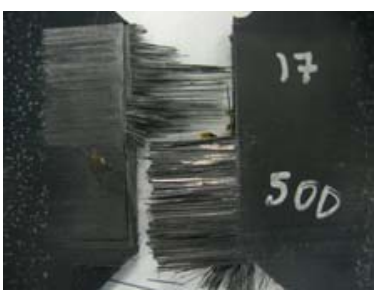

(c) $500 \mathrm{in} / \mathrm{sec}$

Figure 31. Failure modes in Newport Unitape material at different stroke rates 


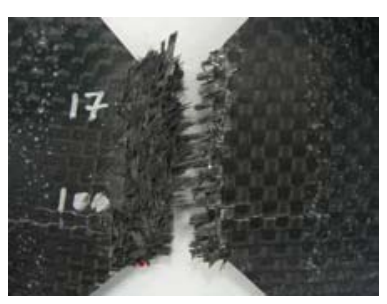

(a) $100 \mathrm{in} / \mathrm{sec}$

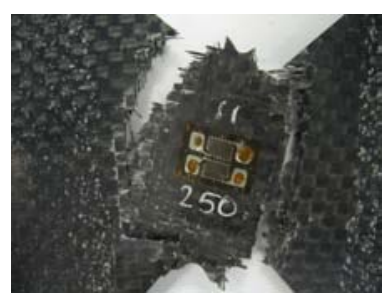

(b) $250 \mathrm{in} / \mathrm{sec}$

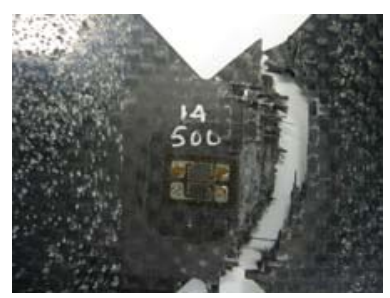

(c) $500 \mathrm{in} / \mathrm{sec}$

Figure 32. Failure modes in Newport PWCF material at different stroke rates.

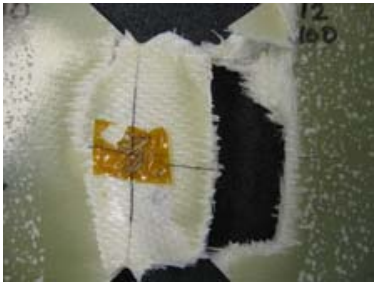

(a) $100 \mathrm{in} / \mathrm{sec}$

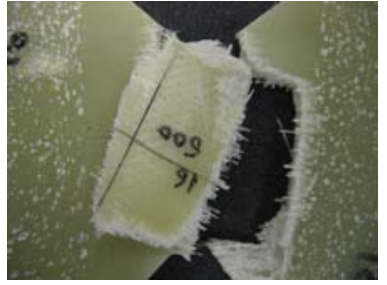

(b) $250 \mathrm{in} / \mathrm{sec}$

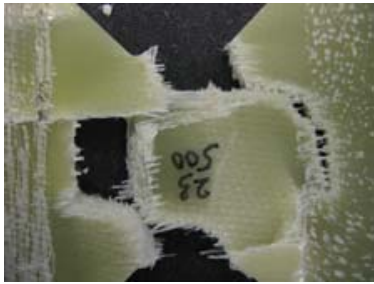

(c) $500 \mathrm{in} / \mathrm{sec}$

Figure 33. Failure modes in Newport SWGF material at different stroke rates

The fracture planes for Newport SWGF specimens were located away from the minimum section at all loading rates. Unlike the Newport PWCF material, the fracture paths passed through the notch at rates up to $100 \mathrm{in} / \mathrm{sec}$. The ultimate shear strengths which are computed using the minimum cross-section will be much higher for the specimens failing away from the minimum cross-section. In order to enforce failure across the minimum section at higher rates, a deeper notch may have to be used. For that reason a Newport SWGF specimen with 0.5 inch gage length (deeper notch) was tested at $500 \mathrm{in} / \mathrm{sec}$ rate. The specimen geometry is shown in the Figure 34. The failure mode was seen across the minimum section of the specimen. 


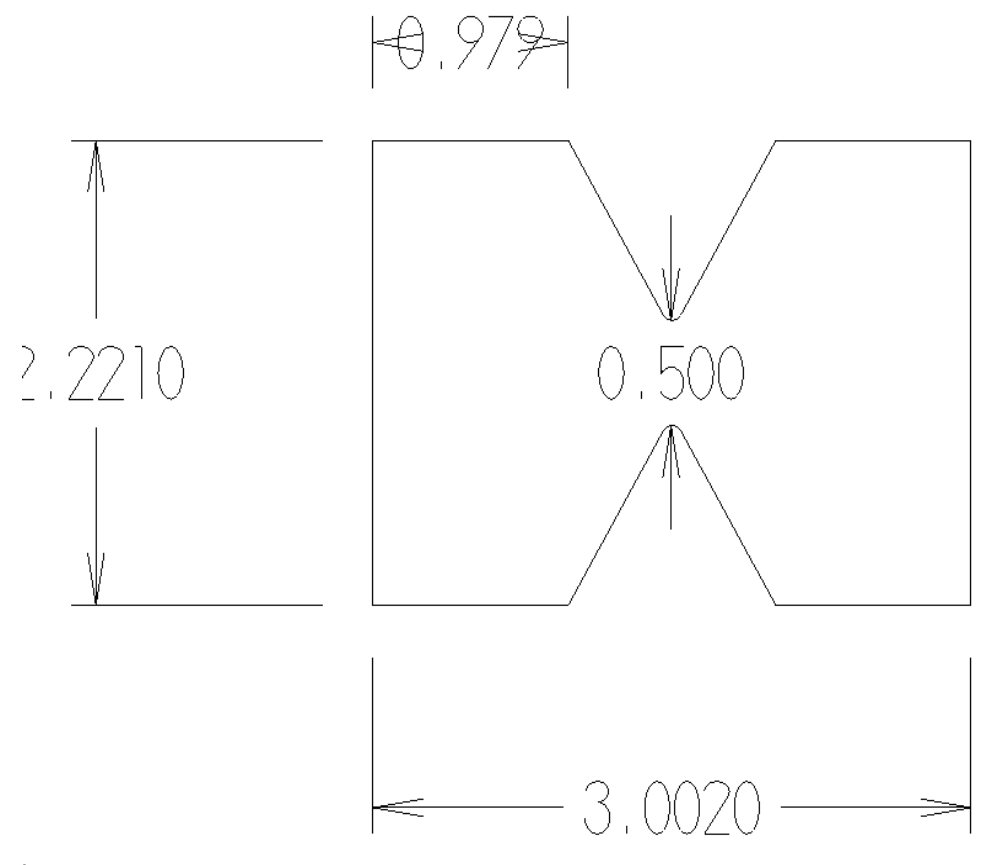

Figure 34. Specimen Geometry with deeper notch.

The failure stress decreased with a decrease in the gage length and there was more than two times increase in average strain rate with 0.5 in gage length than with 1.22 gage length.(from Table 6.)

Table 7

Comparison of test data for Newport SWGF material at 500in/sec

\begin{tabular}{|c|c|c|c|c|}
\hline $\begin{array}{c}\text { Gage Length } \\
\text { (in) }\end{array}$ & $\begin{array}{c}\text { Nominal } \\
\text { Stroke Rate } \\
(\mathrm{in} / \mathrm{s})\end{array}$ & $\begin{array}{c}\text { Ultimate Failure } \\
\text { Stress } \\
\left(\mathrm{lbf} / \mathrm{in}^{2}\right)\end{array}$ & $\begin{array}{c}\text { Average Strain } \\
\text { Rate } \\
(\mathrm{rad} / \mathrm{sec})\end{array}$ & $\begin{array}{c}\text { Strength } \\
(\mathrm{lbs})\end{array}$ \\
\hline 1.22 & 500 & 46621.61 & 375 & 7490.23 \\
\hline 0.5 & 500 & 42661.34 & 890.5 & 2778.32 \\
\hline
\end{tabular}


The strength of the material decreased as the area of the notch decreased. The stressstrain and strain rate-strain was compared for both the gage lengths (shown in the Figure 35 and 36). The stiffness of the material was higher for 1.22in gage length specimen and the strain rate increased with a decrease in the notch area.

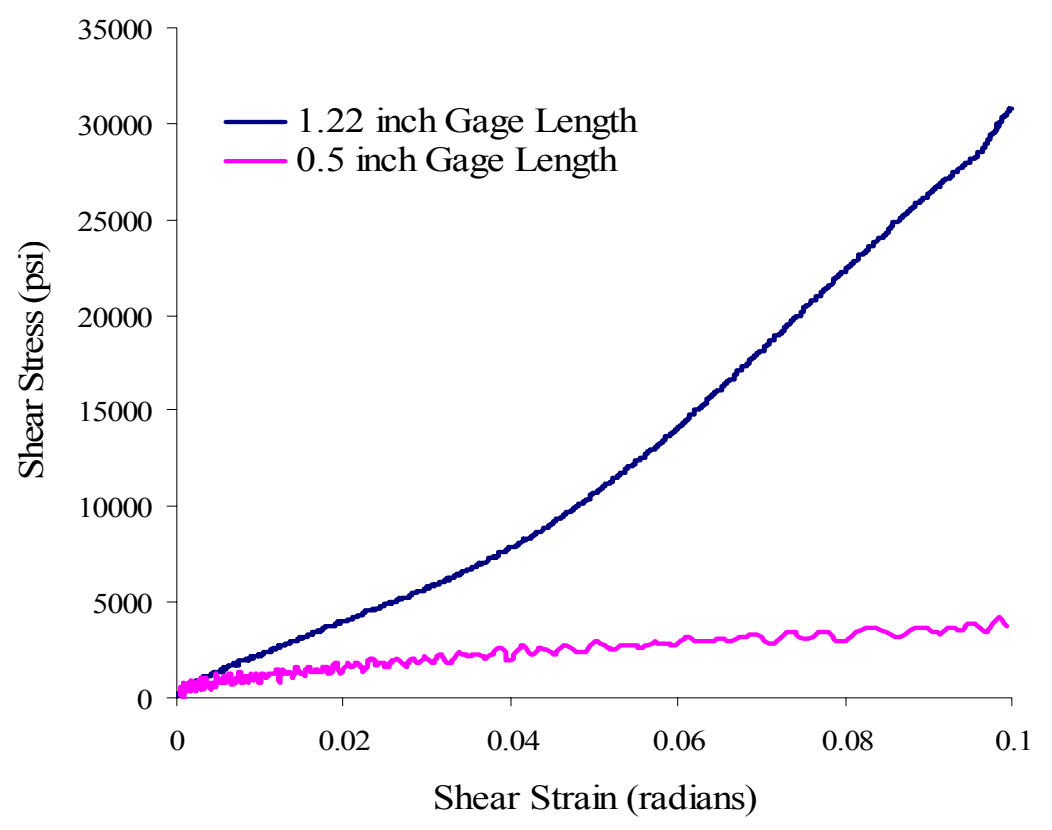

Figure 35. Comparison of shear stress-strain between two gage lengths.

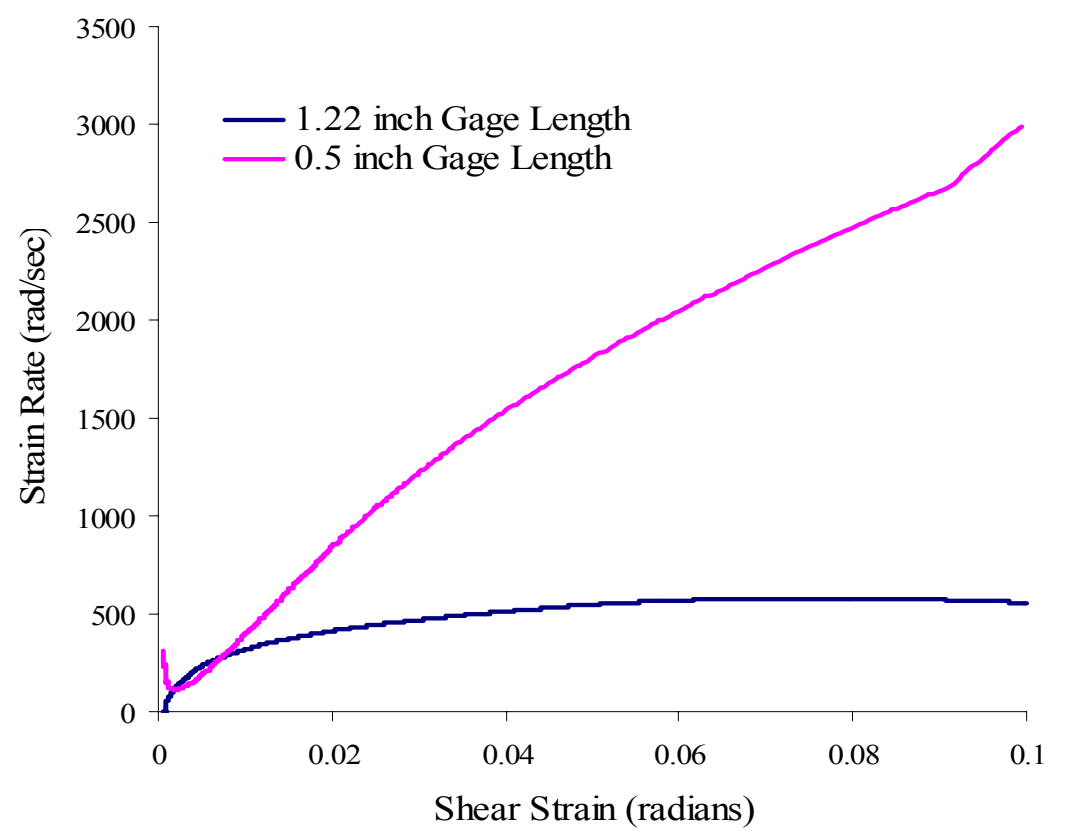

Figure 36. Comparison of strain rate-strain between two gage lengths. 


\section{CHAPTER 7}

\section{CONCLUSIONS AND RECOMMENDATIONS}

\subsection{Conclusion:}

The effects of strain rate on the in-plane shear behavior of Newport materials, Cytec PWC/T300/3KNT and Fibercote 3KPW/E365 material systems were investigated experimentally. The testing was conducted using a servo hydraulic at nominal stroke rates ranging between $0.00083 \mathrm{in} / \mathrm{sec}$ to $500 \mathrm{in} / \mathrm{sec}$. A maximum strain rate of $654 \mathrm{rad} / \mathrm{sec}$ was achieved up to shear strain levels of 0.08 radians, during the tests. The stress-strain plots of all material systems exhibited contrasting behavior with increasing stroke rates. The stress-strain curves exhibited an asymptotic behavior for stroke rates approaching $100 \mathrm{in} / \mathrm{sec}$ and were dependent on the material systems at stroke rate exceeding $250 \mathrm{in} / \mathrm{sec}$. An appreciable reduction in stiffness was observed with increasing stroke rates, within the shear strain range of 0.02 radians. For Newport NB321/3k70 and NB321/7781 at the highest test rate, the shear strengths increased by a factor of three relative to that of the quasi-static rate, and were independent of the reinforcement type. The shear strength for Cytec PWC/T300/3KNT, Fibercote 3KPW/E365 and Newport NCT/321/G150 increased up to a stroke rate of $100 \mathrm{in} / \mathrm{sec}$ and later decreased with increasing stroke rate. The failure modes for Cytec PWC/T300/3KNT, Fibercote 3KPW/E365 and Newport NCT/321/G150 were at the minimum section of the shear coupons, while for Newport NB321/3k70 and NB321/7781, failure was observed to change from a shear mode across the minimum section to a complex failure mode away from the minimum section. 


\subsection{Recommendations:}

Based on the observation from the present study, the following recommendations are made for future work.

1. The results showed an increasing and decreasing trend of material strength at higher stoke rates, further study should identify whether the issue is a material property or a system dynamic problem, and make suitable corrections.

2. In case of the test set-up, there should be enough damping provided in between the slack adapter and the engaging pin so as to reduce the vibrations and data scatter in higher stroke rate tests.

3. Future focus can be emphasized on the development of better high rate test standards/fixtures for evaluation of dynamic material properties of composites. 


\section{REFERENCES}




\section{LIST OF REFERENCES}

[1] ASTM D7078/D7078M-05, "Standard Test Method for Shear Properties of Composite Materials by V-notched Rail Shear Method", ASTM Book of Standards Vol.15.03, 2005.

[2] Daniel O. Adams, Joseph M. Moriarty and Adam M. Gallegos, " The V-Notched Rail Shear Test", Department of Mechanical Engineering, University of Utah, Salt Lake city, UT 84112,USA.

[3] Okolio. I, Smith G. F., "High strain rate characterization of a glass/epoxy composite", American Society for Testing and Materials, Philadelphia, PA.

[4] C.Kammerer and A.Neme, "Plane behavior of E-glass/polyester composites at high strain rates".

[5] Andrew M.Myer and Anthony, "The effect of strain rate on interlaminar shear properties".

[6] B.Bouette, C.Cazeneuve and C.Oytana, L., "Effect of strain rate on interlaminar shear properties of carbon /epoxy composites".

[7] N. Papadakis, N. Reynolds, M. W. Pharaoh, P. K. C. Wood and G. F. Smith, "Strain rate effects on the shear mechanical properties of a highly oriented thermoplastic composite material using a contacting displacement measurement methodology" Composites Science and Technology, Volume 64, Issue 5, April 2004, Pages 729-738

[8] I.W.Hall and M.Guden, " High rate testing of unidirectionally reinforced graphite epoxy composite".

[9] R. Garcia, T.A. Weisshaar and R.R. Mc withey, “An experimental and analytical investigation of rail shear test method as applied to composite materials".

[10] Ahmed K. Hussain and Donald F. Adams, “ Linear three dimensional finite element analyses to evaluate various rail and specimen configurations of two rail shear test procedure".

[11] Konrad Gliesche, Tamara Hübner and Holger Orawetz, "Investigations of in-plane shear properties of $\pm 45^{\circ}$-carbon/epoxy composites using tensile testing and optical deformation analysis", Composites Science and Technology, Volume 65, Issue 2, February 2005, Pages 163-171.

[12] Gary L. Farley and Donald J. Baker, "The in-plane shear test of thin panels".

[13] A. K. Hussain "Experimental Evaluation of the Wyoming-Modified Two-Rail Shear Test Method for Composite Materials".

[14] H.Ho, "In-plane shear testing of graphite woven fabric composites". 
[15] http://www.sae.org/standardsdev

[16] http://www.newportad.com

[17] http://www.cytec.com

[18] http://www.parkelectro.com

[19] MTS Corporation, Eden Prairie, MN.

[20] Vishay Micro Measurements, P.P.Box 27777, Raleigh, NC 26711.

[21] PCB Piezotronics Inc., 3425 Walden Ave., Depew, NY 14043-9902.

[22] National Instruments Corporation, 11500 N Mopac Expwy Austin, TX 78759-3504. 
APPENDIX 


\section{APPENDIX}

As discussed earlier in chapter 6, following are the Stress-Strain and Strain Rate-Strain graphs for Cytec PWC/T300/3KNT material.

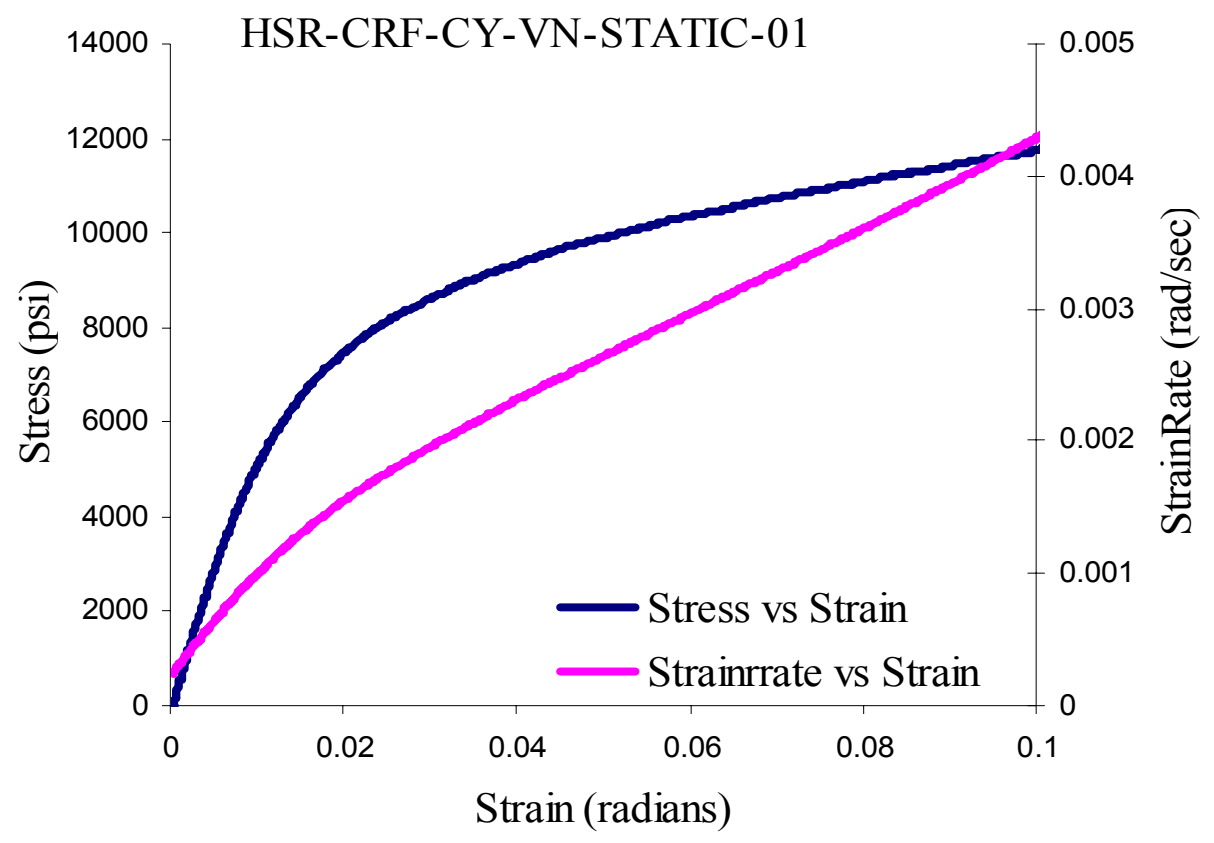

Figure 37. Stress-Strain and Strain Rate-Strain behavior of Cytec at $0.00083 \mathrm{in} / \mathrm{s}$ stroke rate

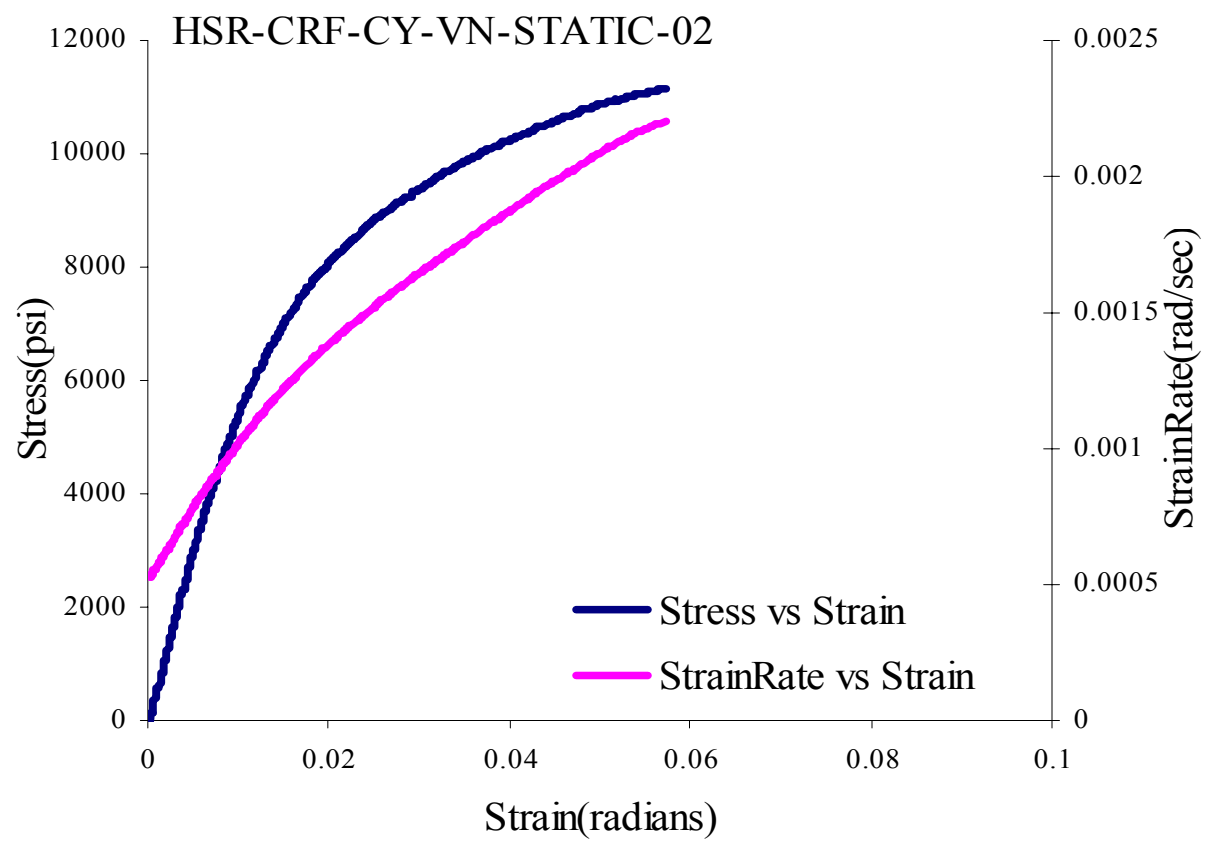


Figure 38. Stress-Strain and Strain Rate-Strain behavior of Cytec at $0.00083 \mathrm{in} / \mathrm{s}$ stroke rate

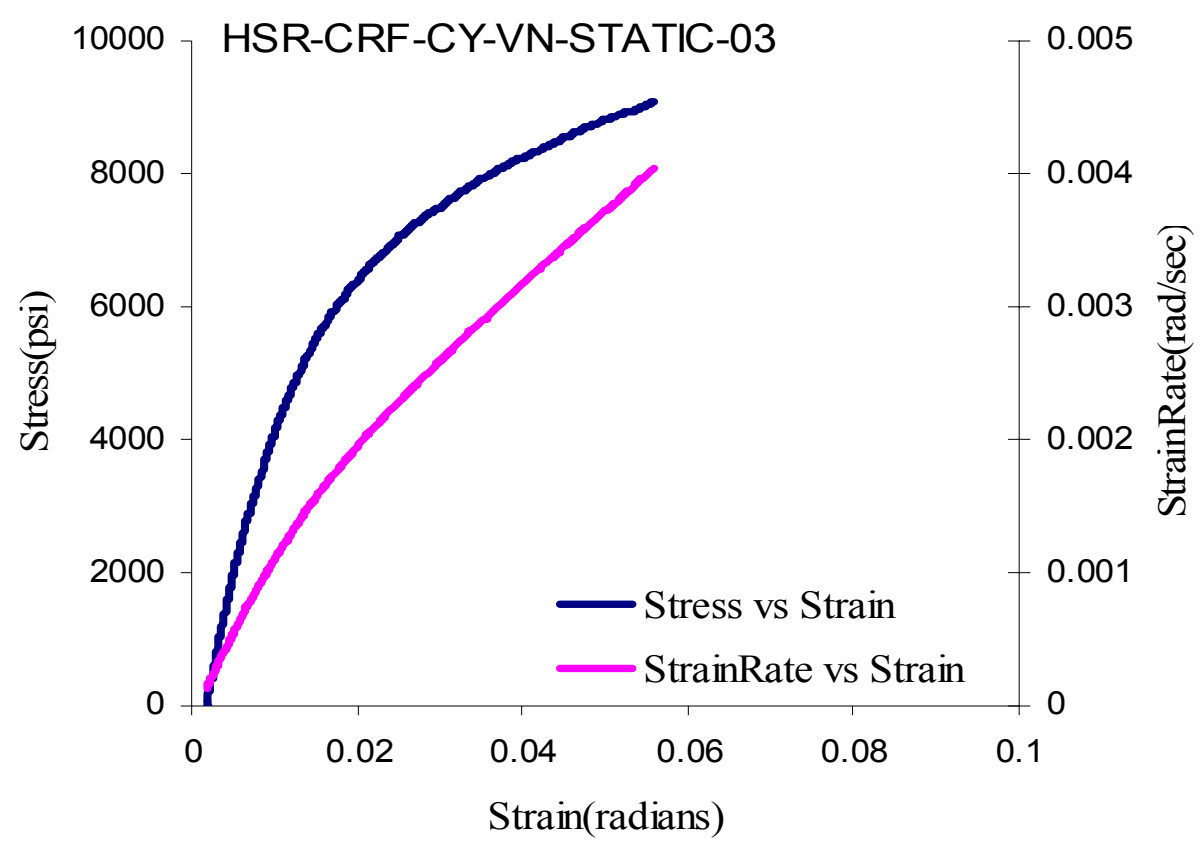

Figure 39. Stress-Strain and Strain Rate-Strain behavior of Cytec at $0.00083 \mathrm{in} / \mathrm{s}$ stroke rate

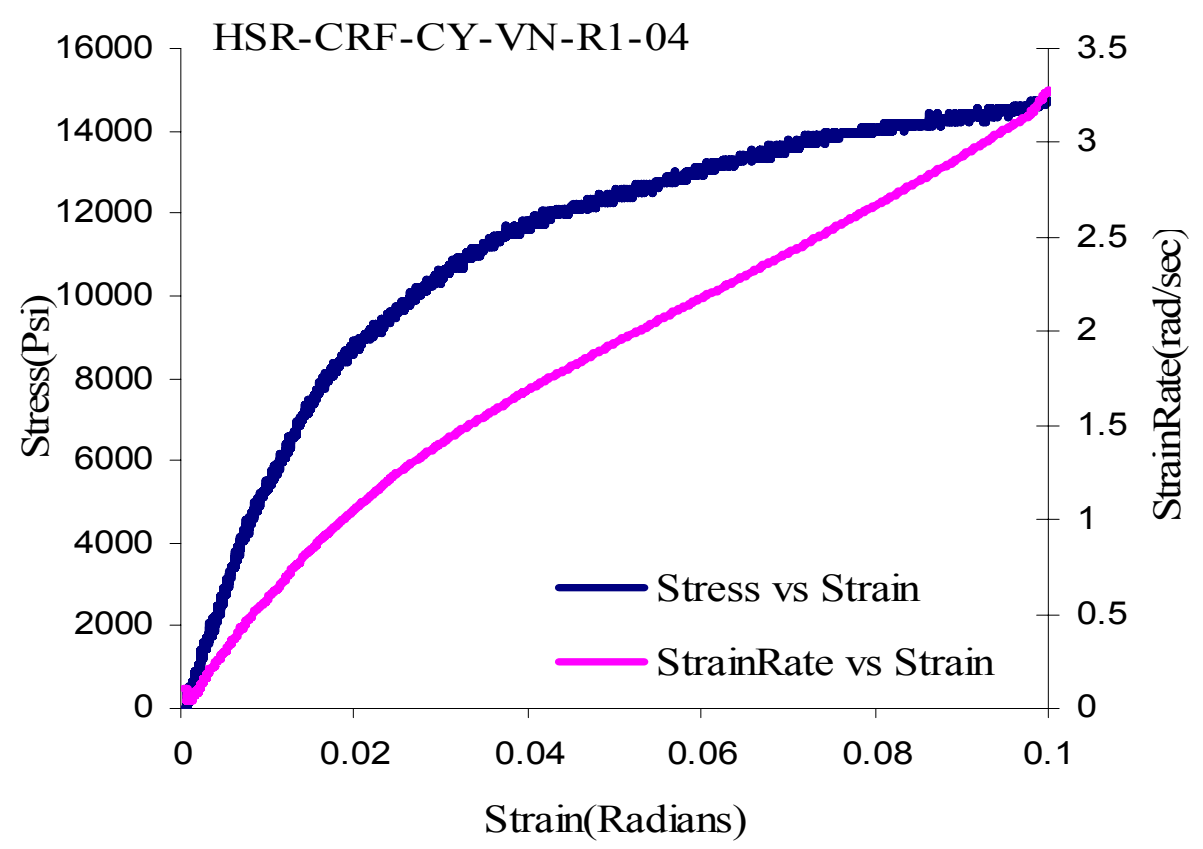

Figure 40. Stress-Strain and Strain Rate-Strain behavior of Cytec at $1 \mathrm{in} / \mathrm{s}$ stroke rate 


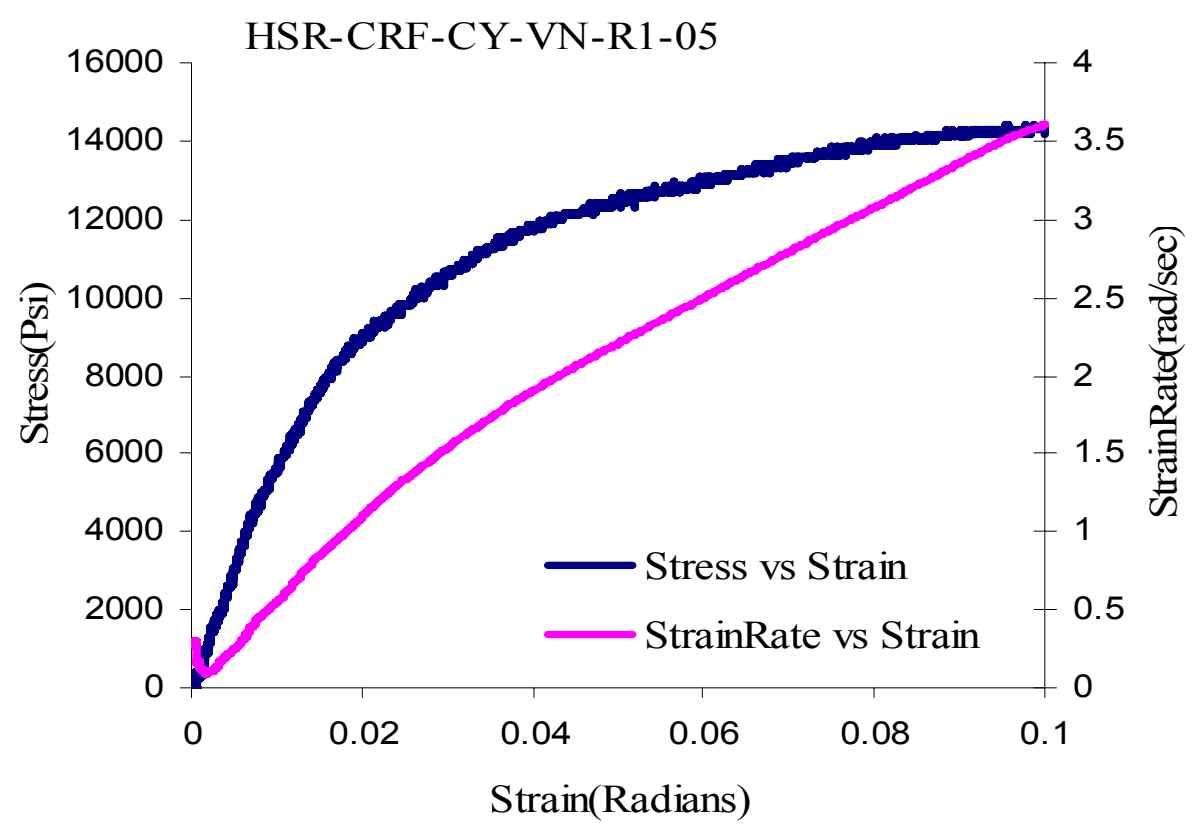

Figure 41. Stress-Strain and Strain Rate-Strain behavior of Cytec at $1 \mathrm{in} / \mathrm{s} \mathrm{stroke} \mathrm{rate}$

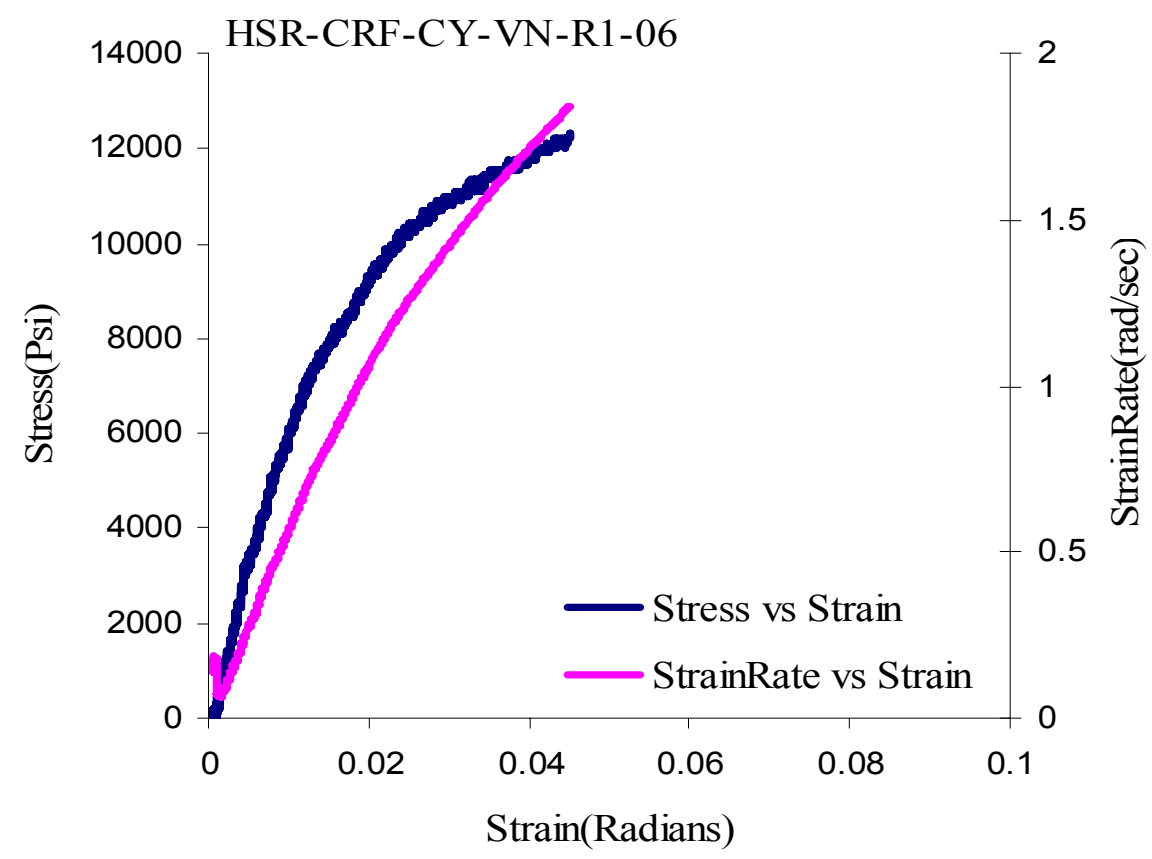

Figure 42. Stress-Strain and Strain Rate-Strain behavior of Cytec at $1 \mathrm{in} / \mathrm{s}$ stroke rate 


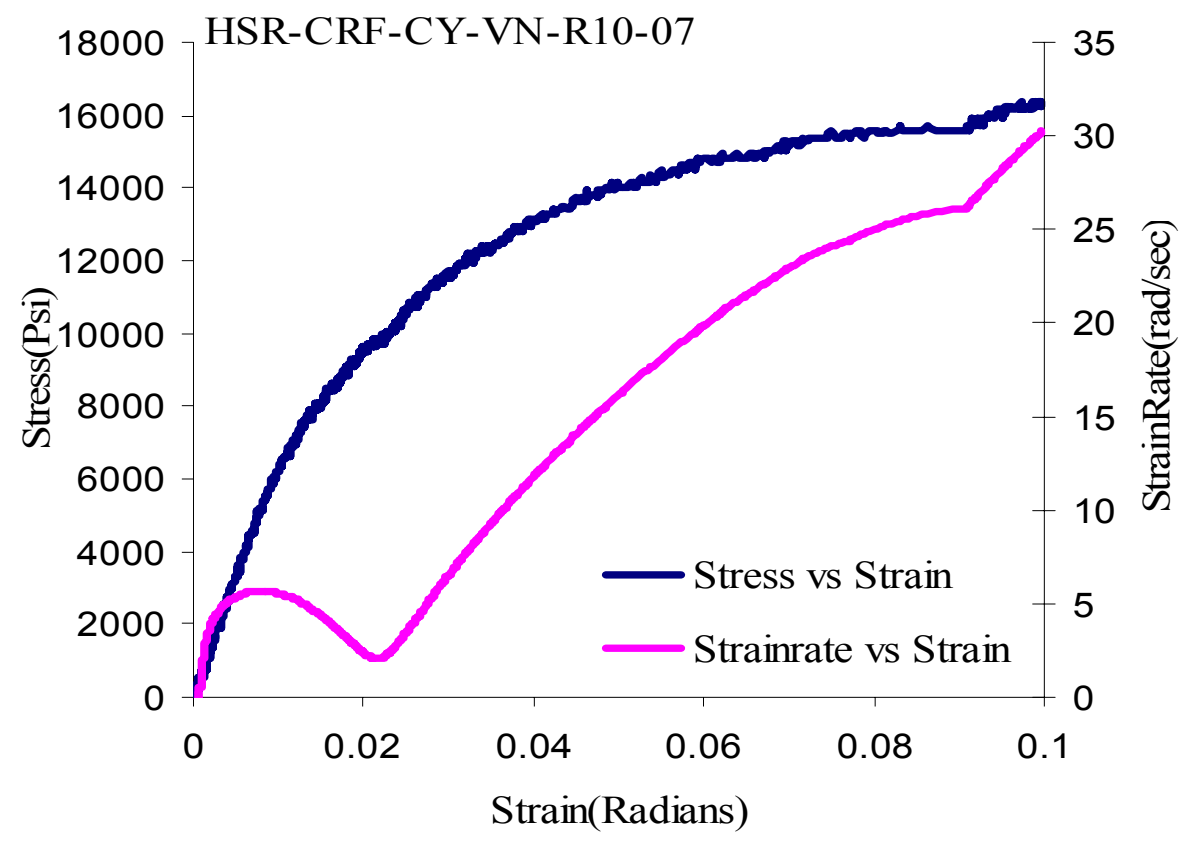

Figure 43. Stress-Strain and Strain Rate-Strain behavior of Cytec at $10 \mathrm{in} / \mathrm{s}$ stroke rate

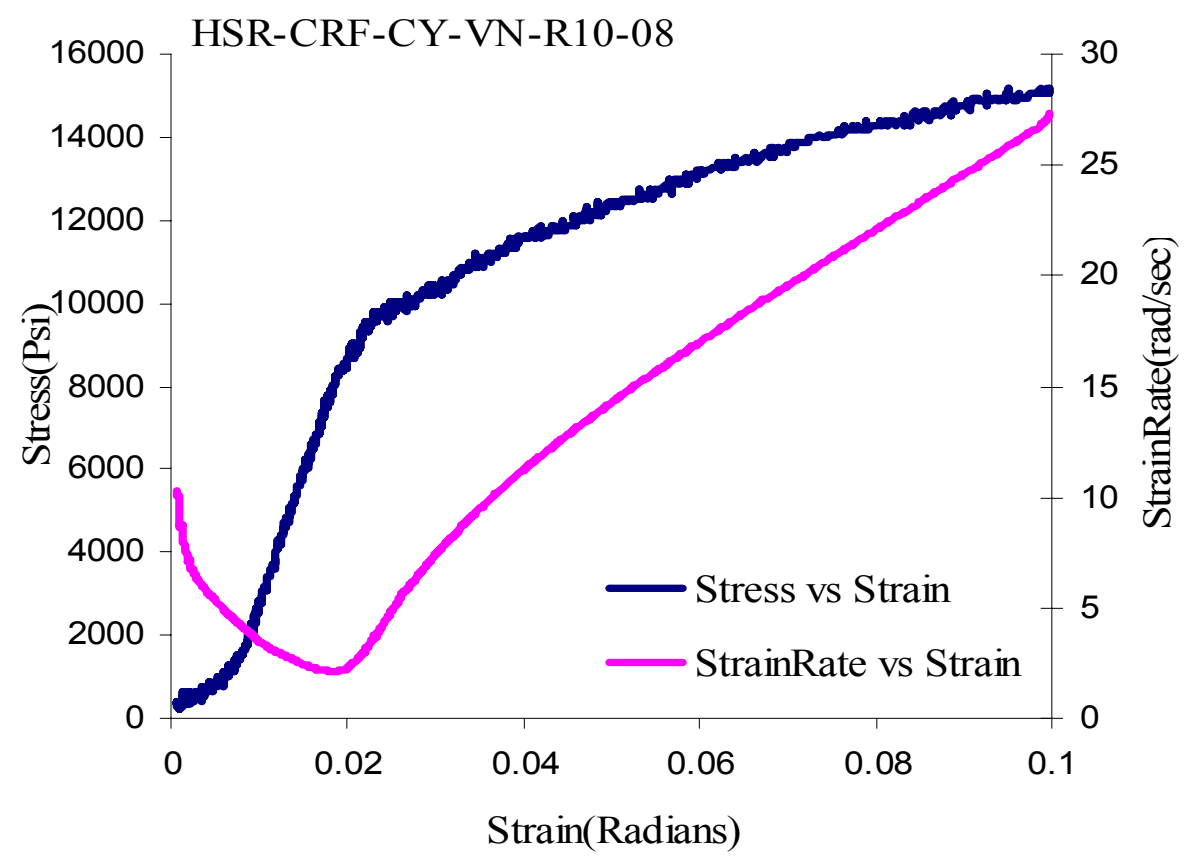

Figure 44. Stress-Strain and Strain Rate-Strain behavior of Cytec at $10 \mathrm{in} / \mathrm{s}$ stroke rate 


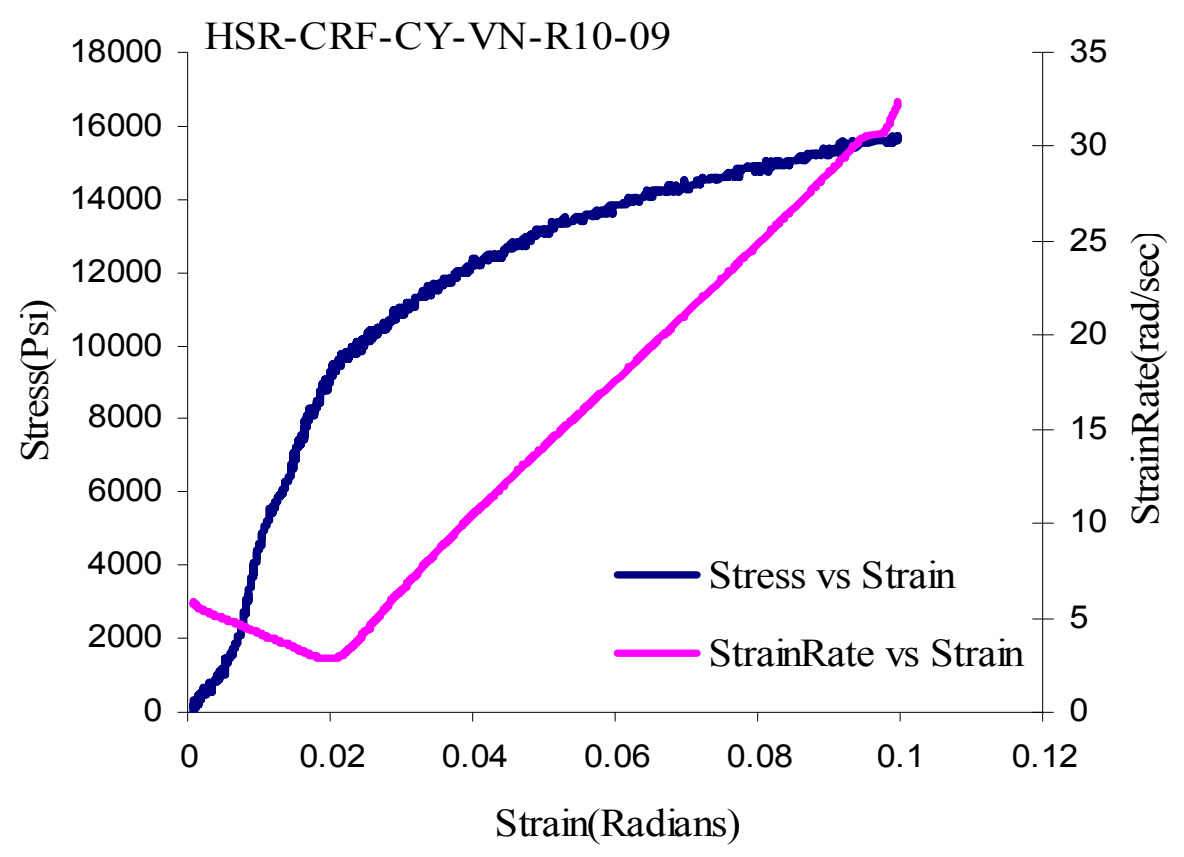

Figure 45. Stress-Strain and Strain Rate-Strain behavior of Cytec at $10 \mathrm{in} / \mathrm{s}$ stroke rate

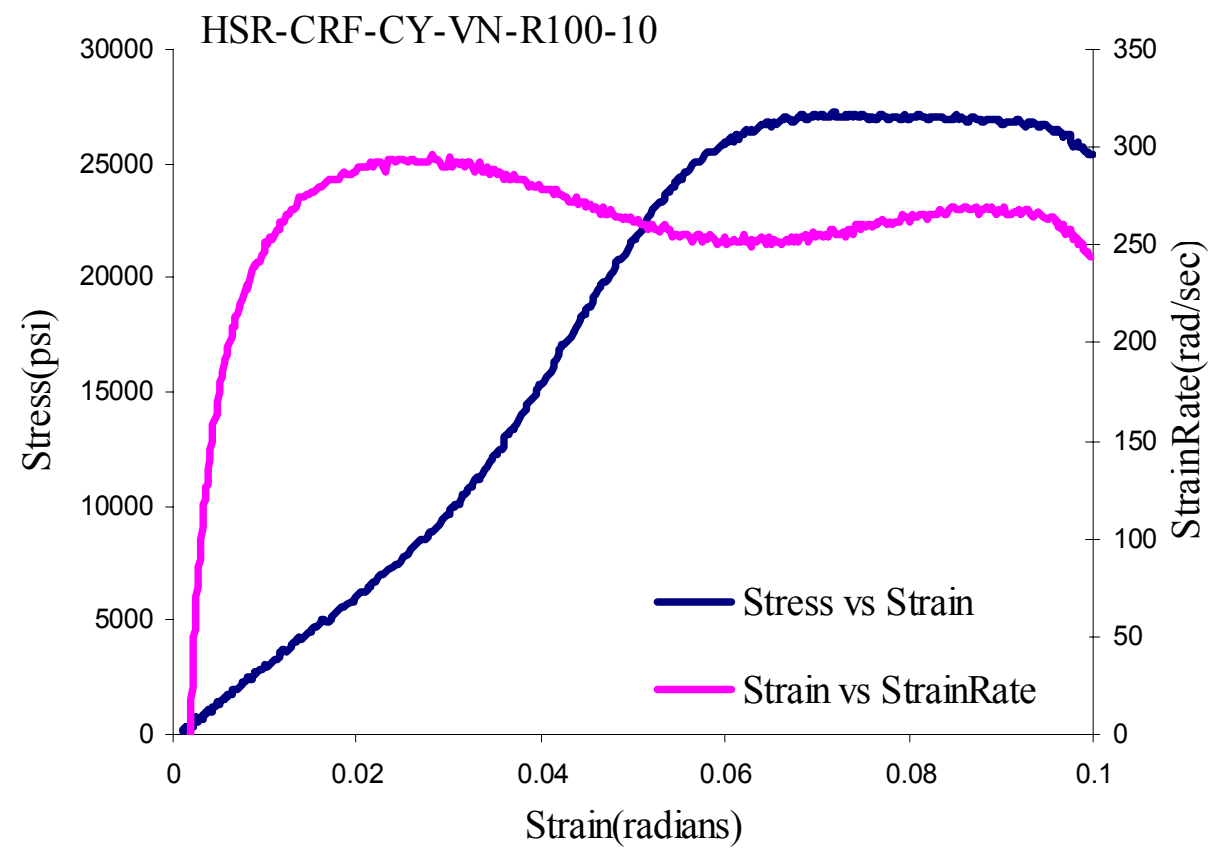

Figure 46. Stress-Strain and Strain Rate-Strain behavior of Cytec at $100 \mathrm{in} / \mathrm{s}$ stroke rate 


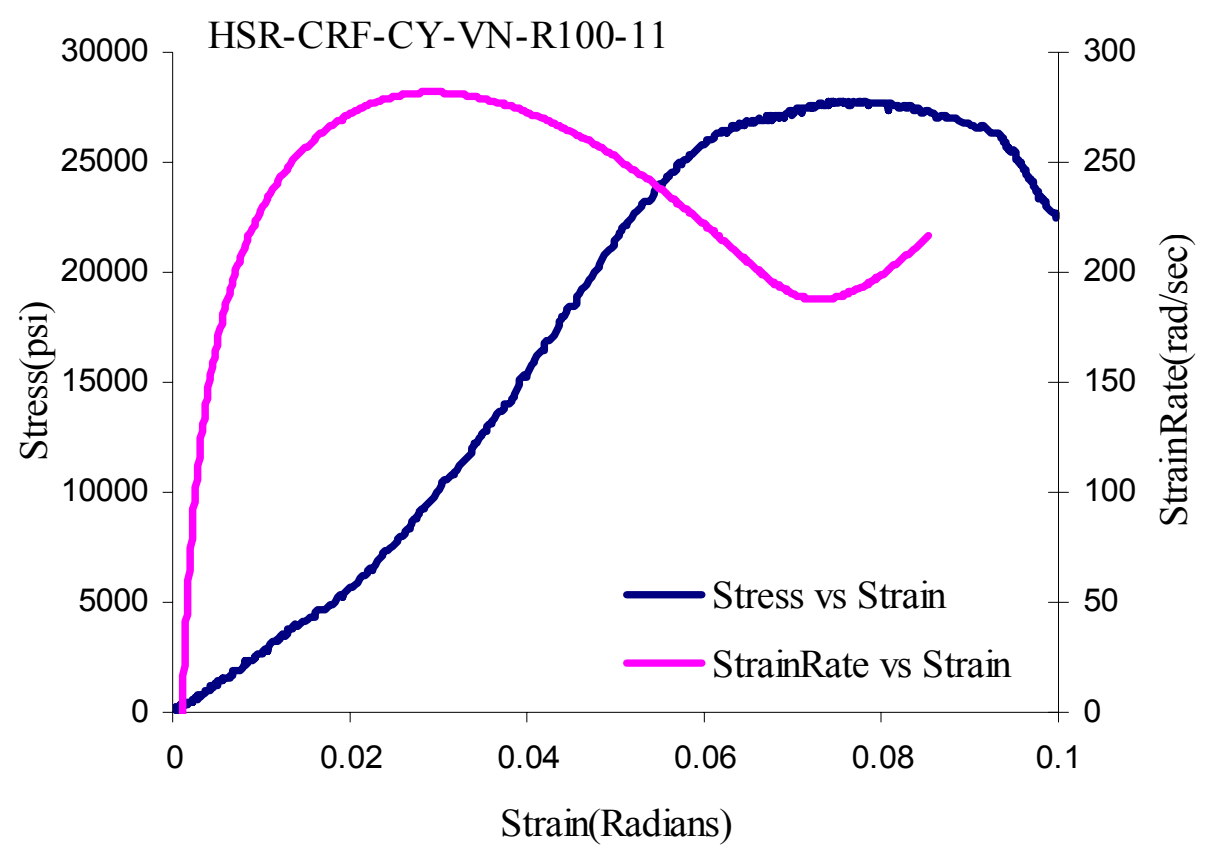

Figure 47. Stress-Strain and Strain Rate-Strain behavior of Cytec at $100 \mathrm{in} / \mathrm{s}$ stroke rate

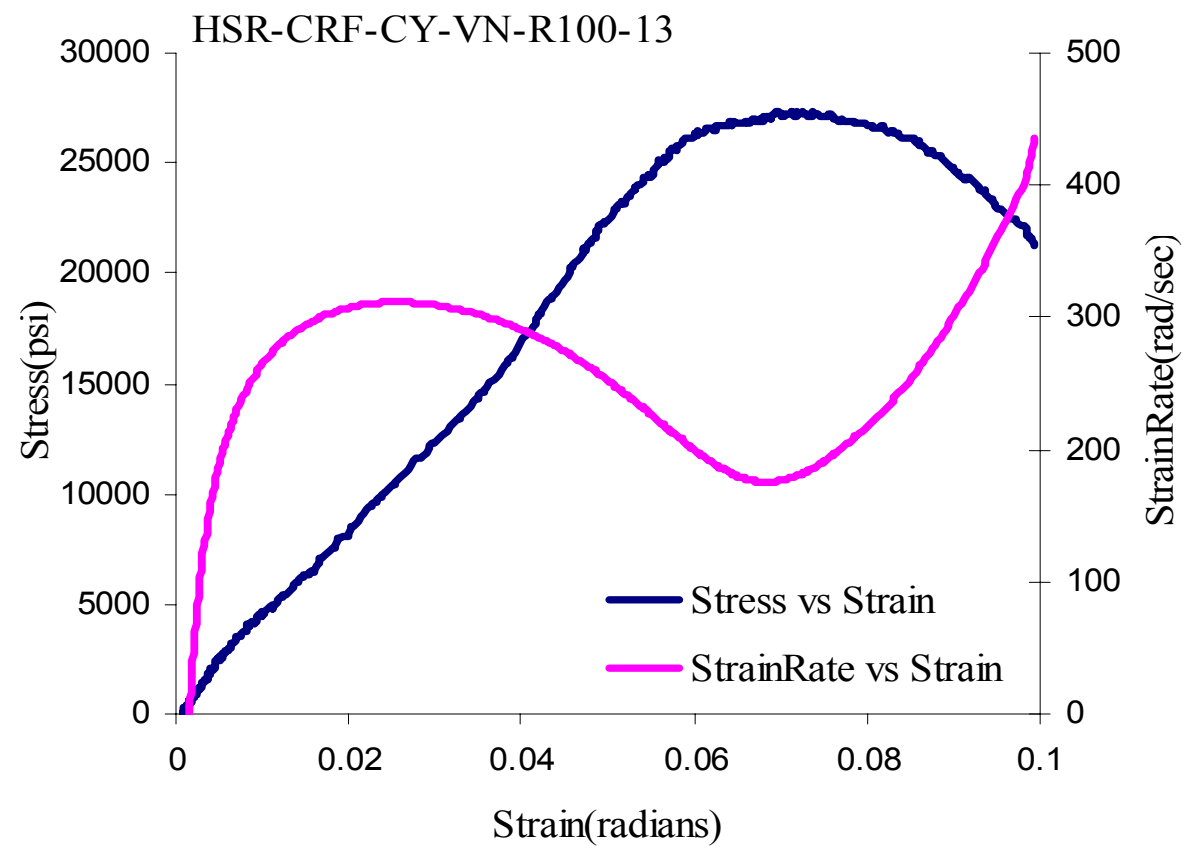

Figure 48. Stress-Strain and Strain Rate-Strain behavior of Cytec at $100 \mathrm{in} / \mathrm{s}$ stroke rate 


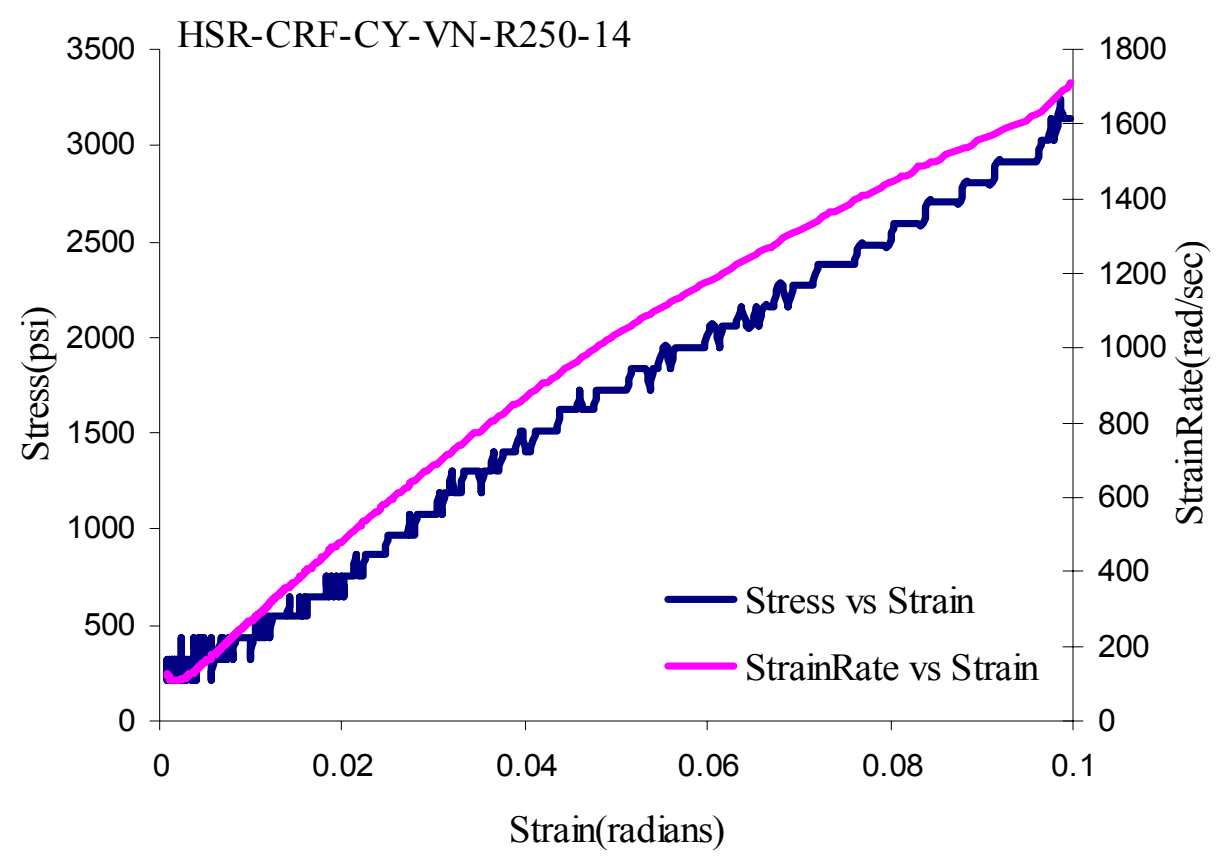

Figure 49. Stress-Strain and Strain Rate-Strain behavior of Cytec at $250 \mathrm{in} / \mathrm{s}$ stroke rate

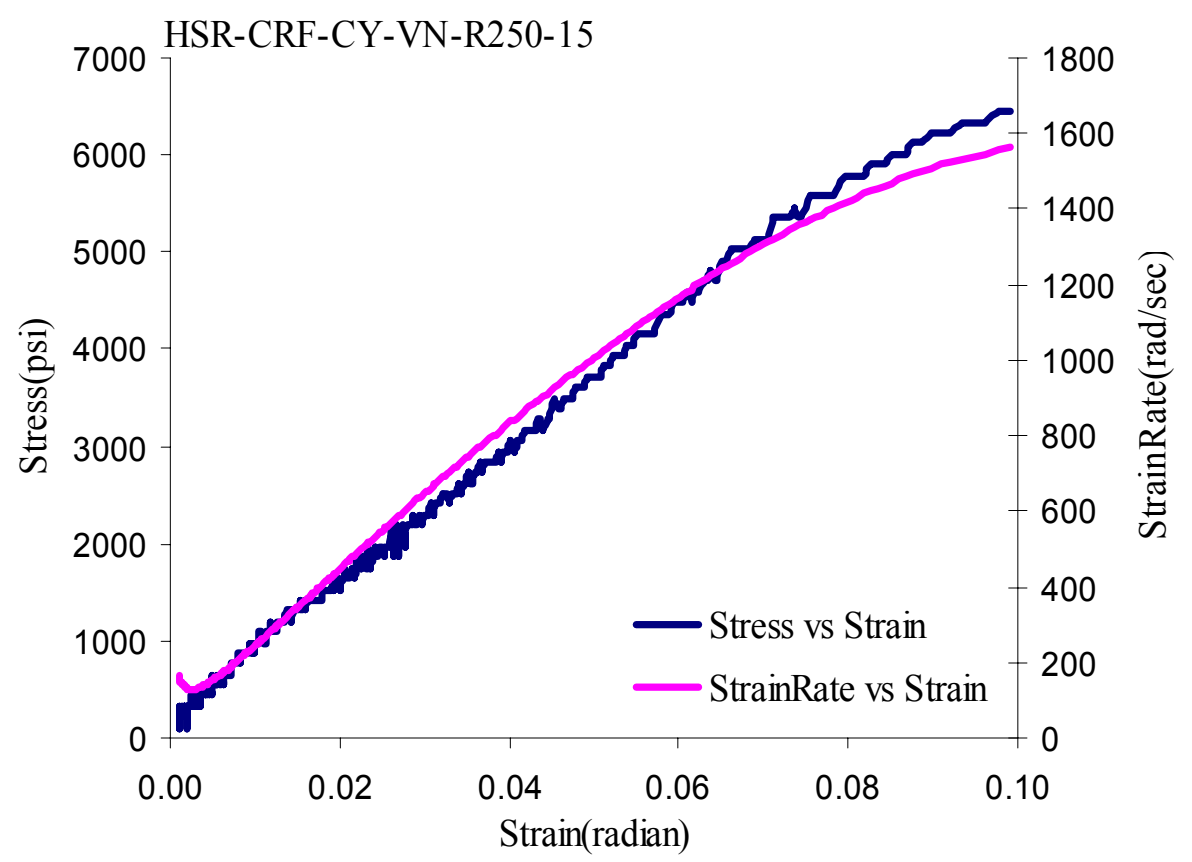

Figure 50. Stress-Strain and Strain Rate-Strain behavior of Cytec at $250 \mathrm{in} / \mathrm{s}$ stroke rate 


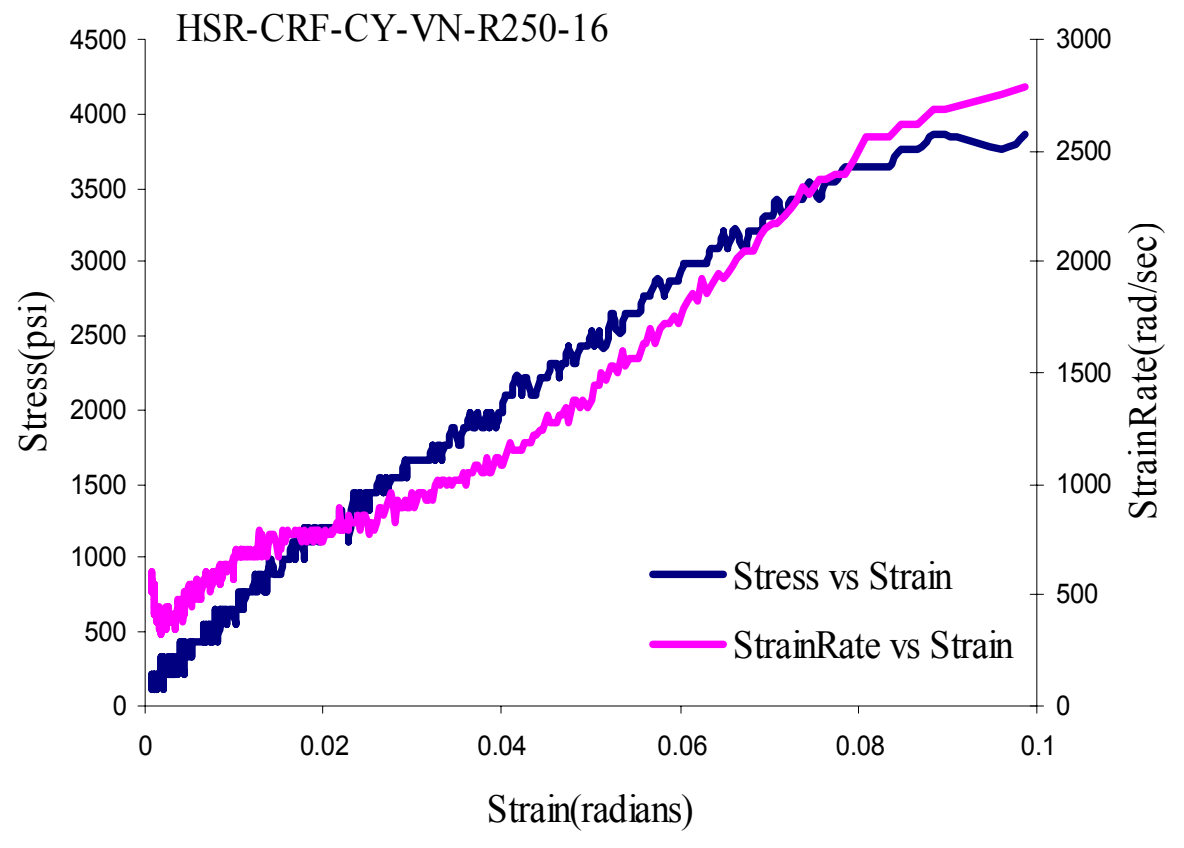

Figure 51. Stress-Strain and Strain Rate-Strain behavior of Cytec at $250 \mathrm{in} / \mathrm{s}$ stroke rate

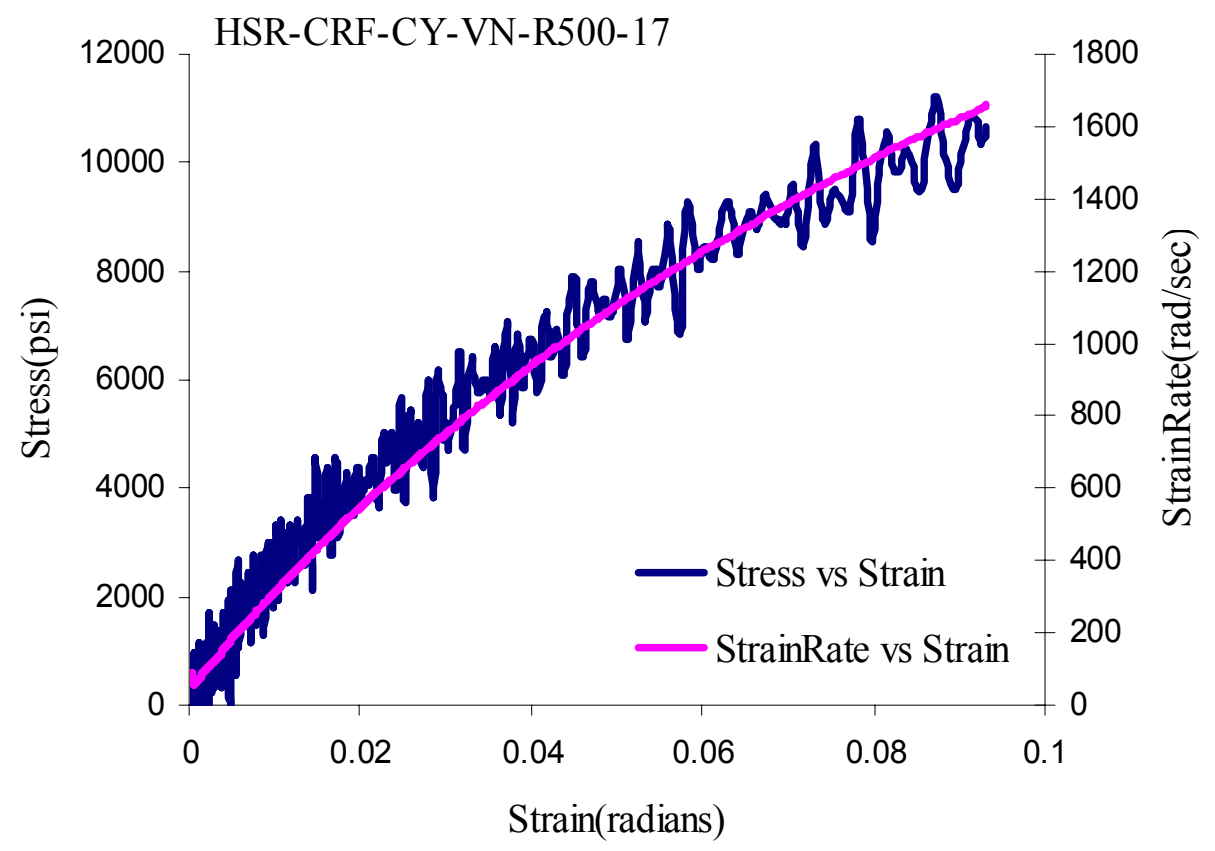

Figure 52. Stress-Strain and Strain Rate-Strain behavior of Cytec at 500 in/s stroke rate 


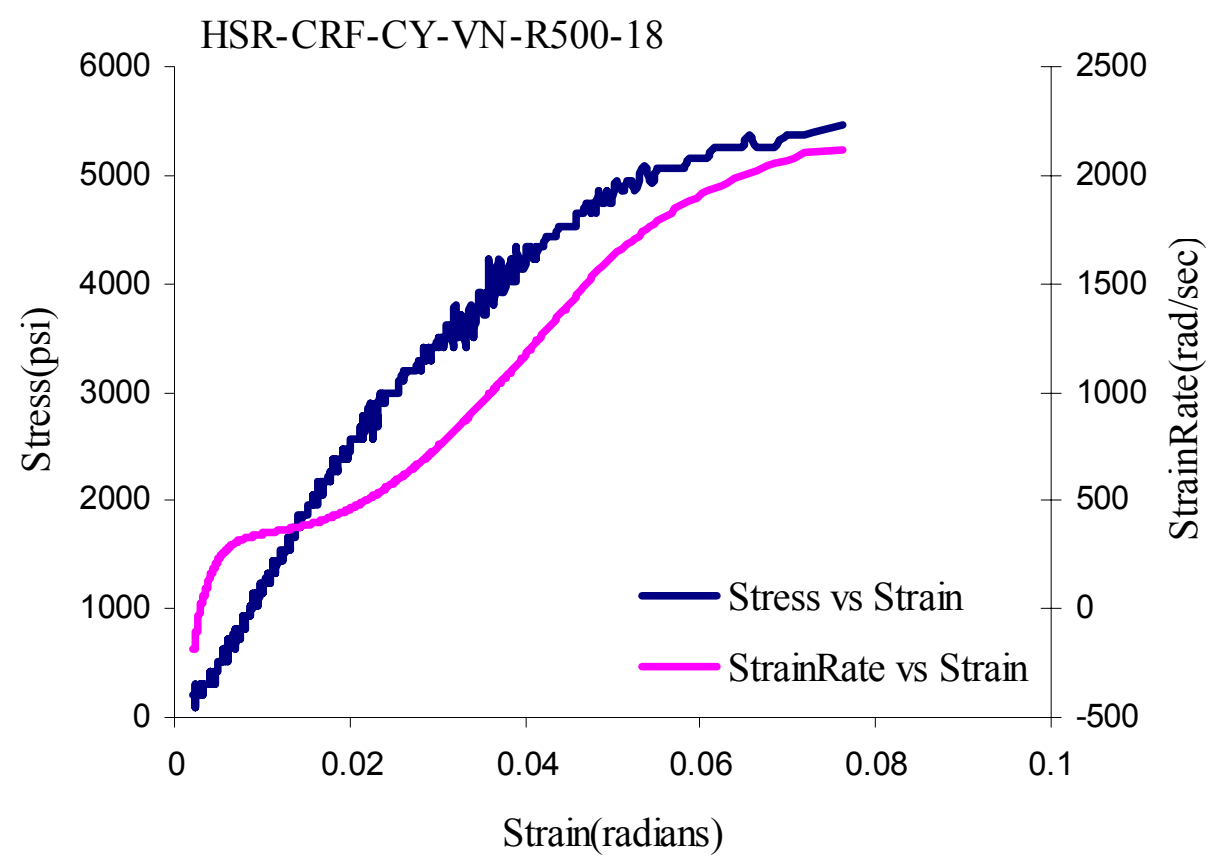

Figure 53. Stress-Strain and Strain Rate-Strain behavior of Cytec at $500 \mathrm{in} / \mathrm{s}$ stroke rate

Failure Patterns of Cytec Specimens at different Stroke Rates

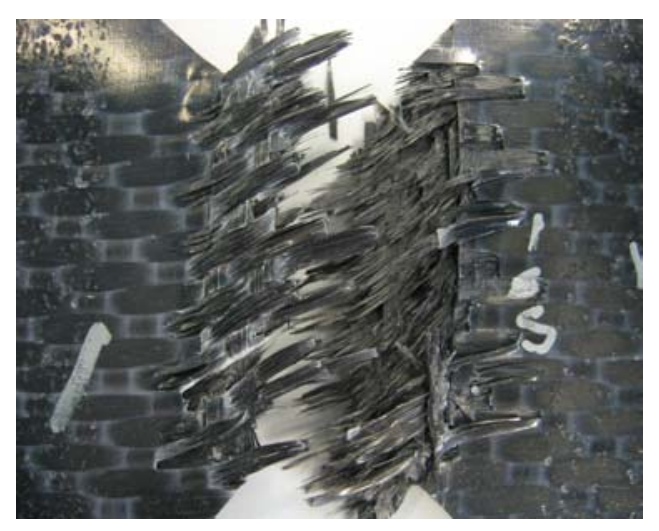

Figure 54. Failure mode of Cytec material at $0.00083 \mathrm{in} / \mathrm{sec}$. 


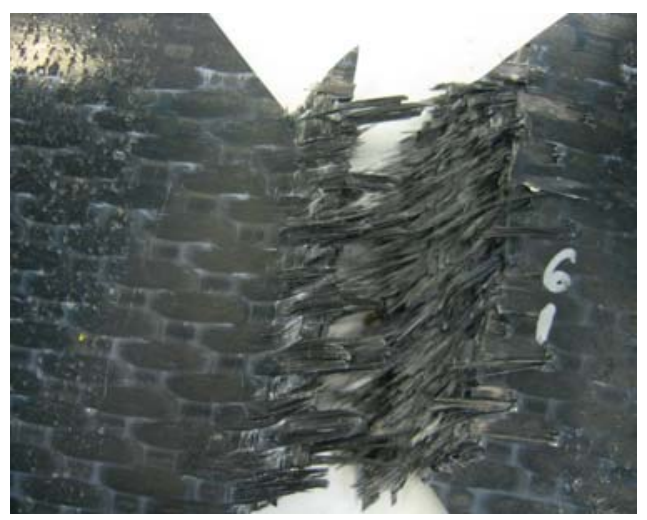

Figure 55. Failure mode of Cytec material at $1 \mathrm{in} / \mathrm{sec}$.

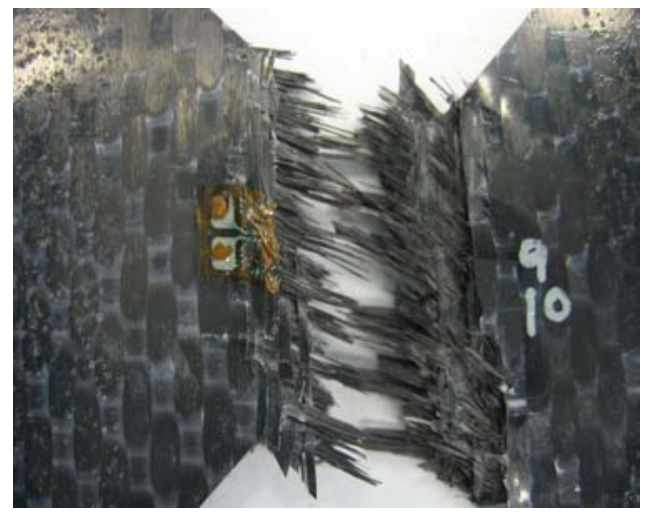

Figure 56. Failure mode of Cytec material at $10 \mathrm{in} / \mathrm{sec}$.

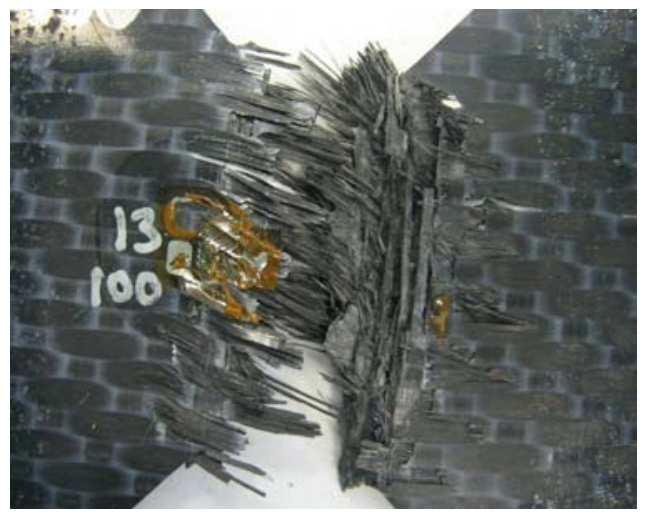

Figure 57. Failure mode of Cytec material at $100 \mathrm{in} / \mathrm{sec}$. 


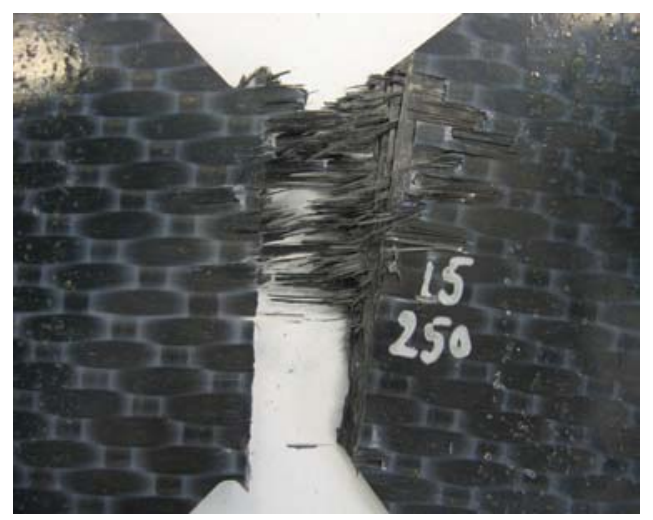

Figure 58. Failure mode of Cytec material at $250 \mathrm{in} / \mathrm{sec}$.

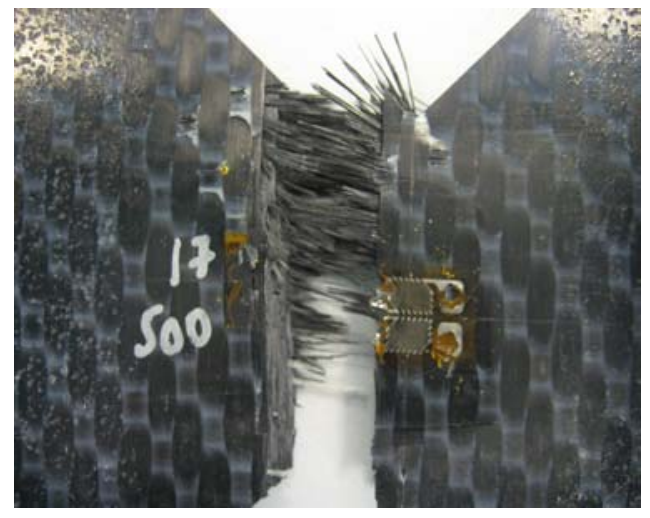

Figure 59. Failure mode of Cytec material at $500 \mathrm{in} / \mathrm{sec}$. 
Table 8

Summary of test data for Cytec material

\begin{tabular}{|c|c|c|c|c|c|}
\hline Specimen \# & $\begin{array}{l}\text { Average } \\
\text { Thickness } \\
\text { (in) }\end{array}$ & $\begin{array}{l}\text { Average Notch } \\
\text { to Notch Width } \\
\text { (in) }\end{array}$ & $\begin{array}{l}\text { Max } \\
\text { Load } \\
(\mathrm{lbf})\end{array}$ & $\begin{array}{l}\text { Failure } \\
\text { Stress } \\
(\mathrm{lbf} / \text { in } 2) \\
\end{array}$ & $\begin{array}{c}\text { Average } \\
\text { Strain Rate } \\
(\mathrm{rad} / \mathrm{sec})\end{array}$ \\
\hline $\begin{array}{l}\text { HSR-CRF-CY-VN-static- } \\
01\end{array}$ & 0.097 & 1.22 & 1947.42 & 16448.1 & 0.0024 \\
\hline $\begin{array}{l}\text { HSR-CRF-CY-VN-static- } \\
02\end{array}$ & 0.095 & 1.22 & 1865.14 & 16075.37 & 0.0013 \\
\hline $\begin{array}{l}\text { HSR-CRF-CY-VN-static- } \\
03\end{array}$ & 0.093 & 1.22 & 1656.63 & 14549.64 & 0.0037 \\
\hline HSR-CRF-CY-VN-R1-04 & 0.095 & 1.22 & 2196.28 & 18779.24 & 0.98 \\
\hline HSR-CRF-CY-VN-R1-05 & 0.093 & 1.22 & 2132.81 & 18634.99 & 1.05 \\
\hline HSR-CRF-CY-VN-R1-06 & 0.093 & 1.22 & 2120.11 & 18556.01 & 0.54 \\
\hline HSR-CRF-CY-VN-R10-07 & 0.093 & 1.22 & 2412.1 & 21026.05 & 8.25 \\
\hline HSR-CRF-CY-VN-R10-08 & 0.094 & 1.22 & 2500.97 & 21669.04 & 8.48 \\
\hline HSR-CRF-CY-VN-R10-09 & 0.093 & 1.22 & 2361.32 & 20589.32 & 8.51 \\
\hline $\begin{array}{l}\text { HSR-CRF-CY-VN-R100- } \\
10\end{array}$ & 0.096 & 1.22 & 3948.24 & 33406.73 & 236.94 \\
\hline $\begin{array}{l}\text { HSR-CRF-CY-VN-R100- } \\
11\end{array}$ & 0.096 & 1.22 & 3503.9 & 29603.8 & 201.49 \\
\hline $\begin{array}{l}\text { HSR-CRF-CY-VN-R100- } \\
13\end{array}$ & 0.099 & 1.22 & 3326.17 & 27323.72 & 216.69 \\
\hline $\begin{array}{c}\text { HSR-CRF-CY-VN-R250- } \\
14 \\
\end{array}$ & 0.096 & 1.22 & 2754.88 & 23462.18 & 539.09 \\
\hline $\begin{array}{l}\text { HSR-CRF-CY-VN-R250- } \\
15\end{array}$ & 0.095 & 1.22 & 2856.44 & 24586.8 & 464.81 \\
\hline $\begin{array}{l}\text { HSR-CRF-CY-VN-R250- } \\
16\end{array}$ & 0.094 & 1.22 & 2374.02 & 20666.59 & 888.64 \\
\hline $\begin{array}{l}\text { HSR-CRF-CY-VN-R500- } \\
17\end{array}$ & 0.094 & 1.22 & 3199.21 & 26906.12 & 528.25 \\
\hline $\begin{array}{l}\text { HSR-CRF-CY-VN-R500- } \\
18\end{array}$ & 0.097 & 1.22 & 2043.94 & 362613 & 581.5 \\
\hline $\begin{array}{l}\text { HSR-CRF-CY-VN-R500- } \\
19\end{array}$ & 0.098 & 1.22 & 2907.22 & 24431.5 & 588.83 \\
\hline
\end{tabular}



rates

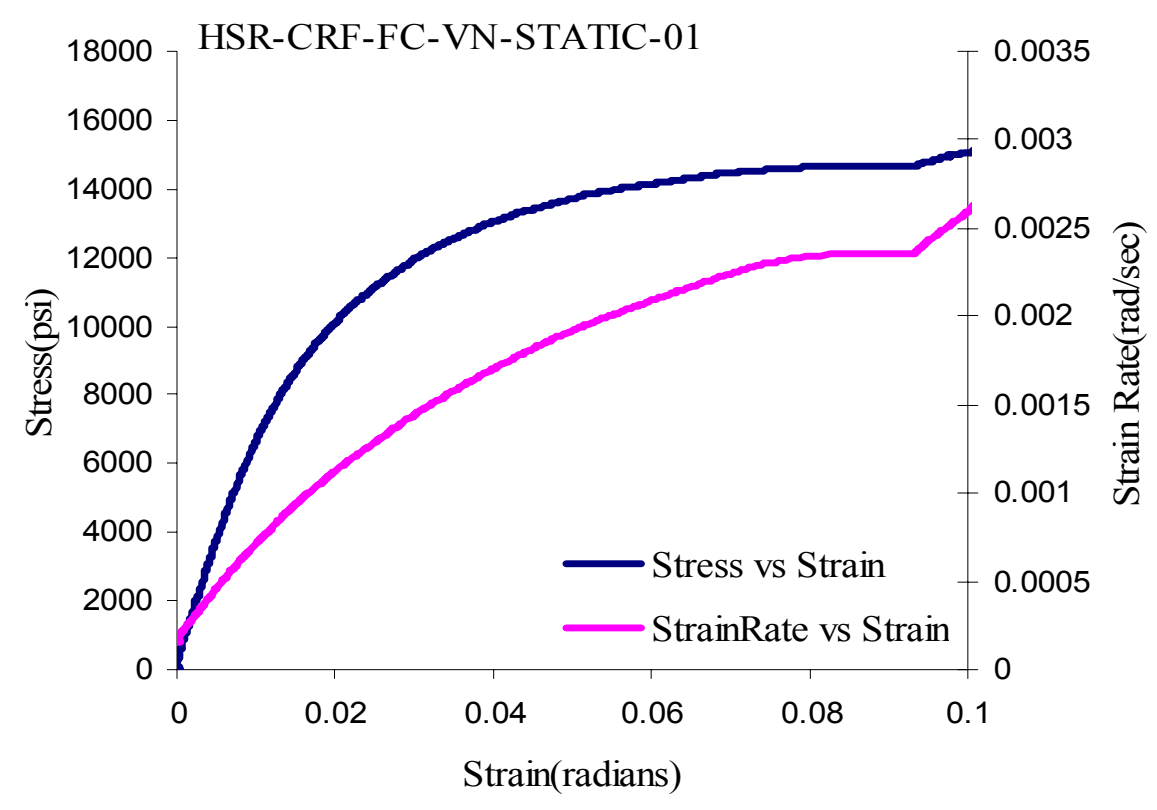

Figure 60. Stress-Strain and Strain Rate-Strain behavior of Fibercote at $0.00083 \mathrm{in} / \mathrm{s}$ stroke rate

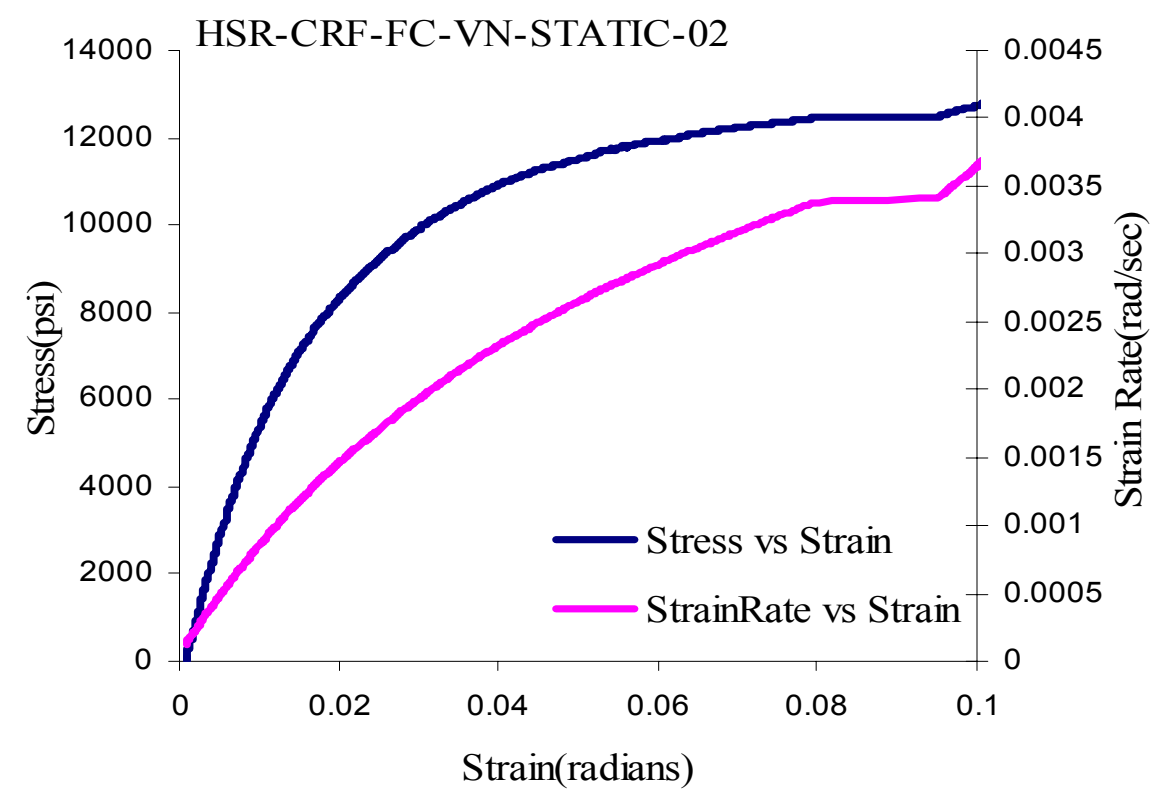

Figure 61. Stress-Strain and Strain Rate-Strain behavior of Fibercote at $0.00083 \mathrm{in} / \mathrm{s}$ stroke rate 


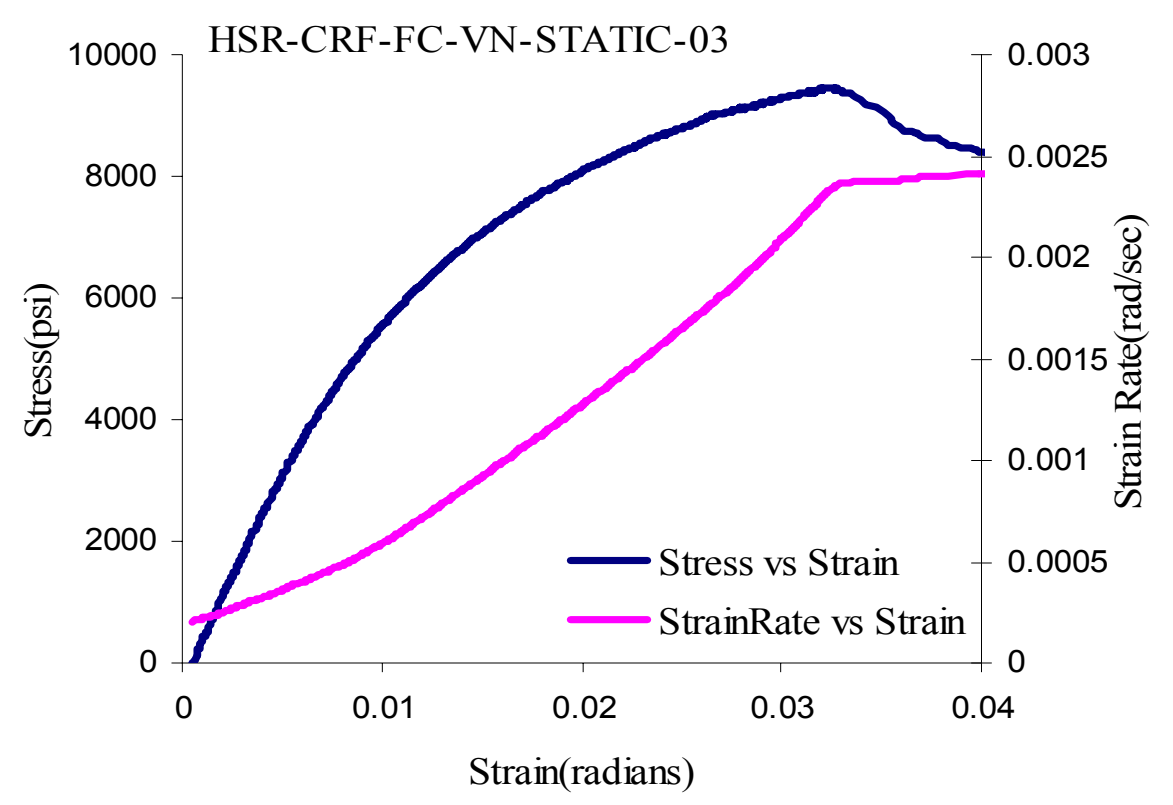

Figure 62. Stress-Strain and Strain Rate-Strain behavior of Fibercote at $0.00083 \mathrm{in} / \mathrm{s}$ stroke rate

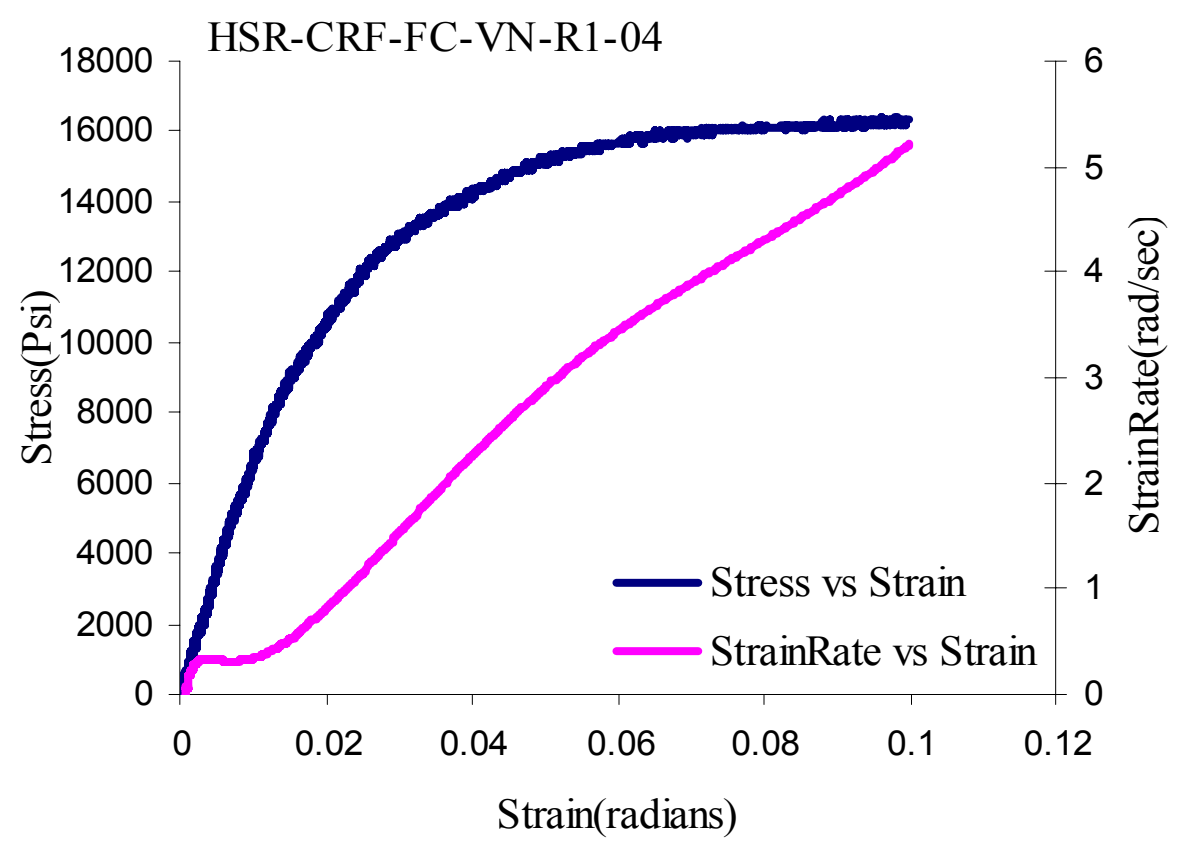

Figure 63. Stress-Strain and Strain Rate-Strain behavior of Fibercote at $1 \mathrm{in} / \mathrm{s}$ stroke rate 


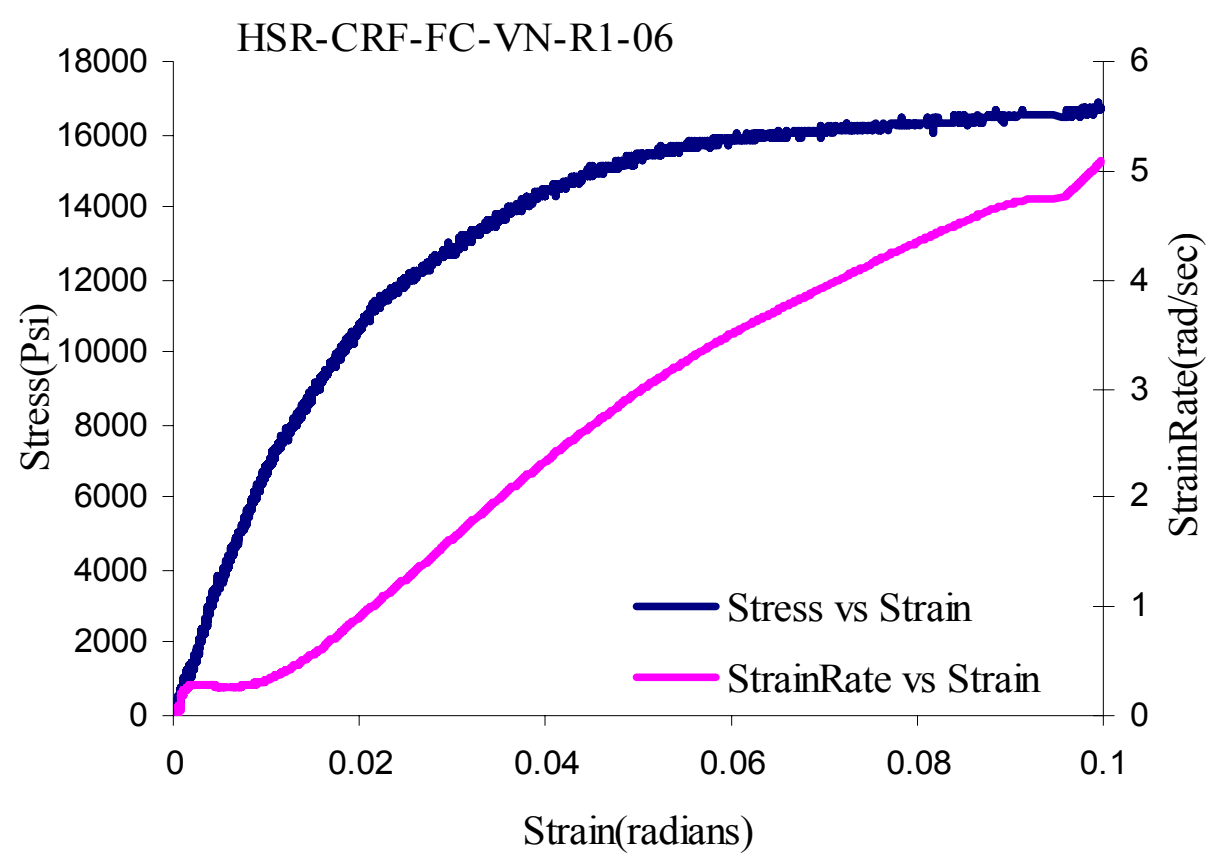

Figure 64. Stress-Strain and Strain Rate-Strain behavior of Fibercote at $1 \mathrm{in} / \mathrm{s}$ stroke rate

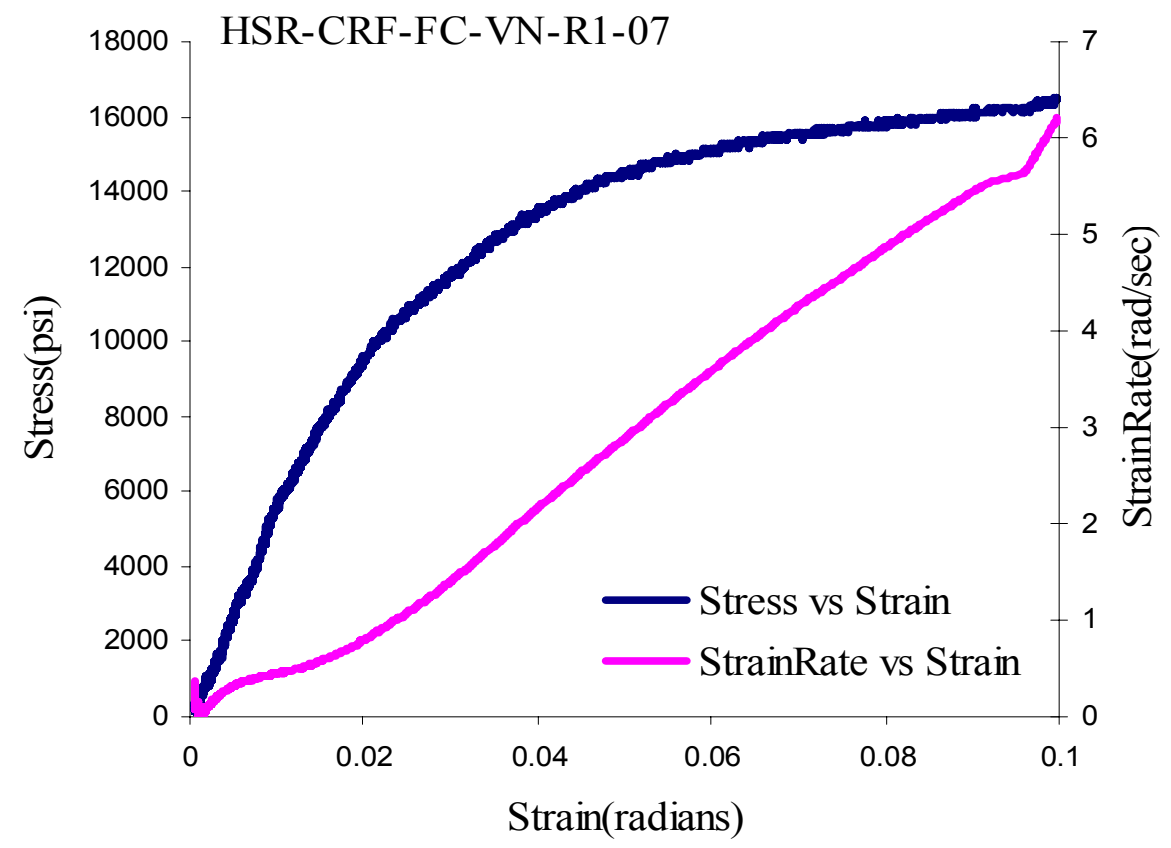

Figure 65. Stress-Strain and Strain Rate-Strain behavior of Fibercote at $1 \mathrm{in} / \mathrm{s}$ stroke rate 


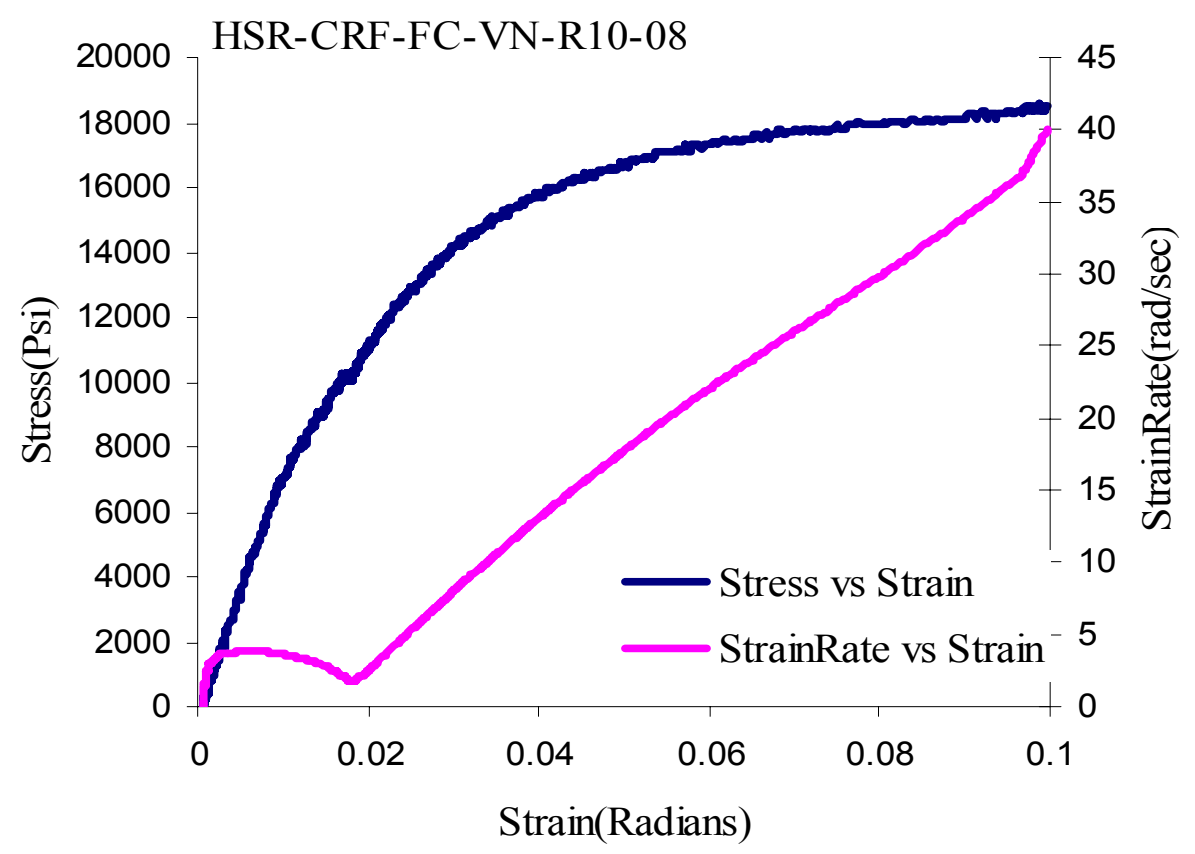

Figure 66. Stress-Strain and Strain Rate-Strain behavior of Fibercote at $10 \mathrm{in} / \mathrm{s}$ stroke rate

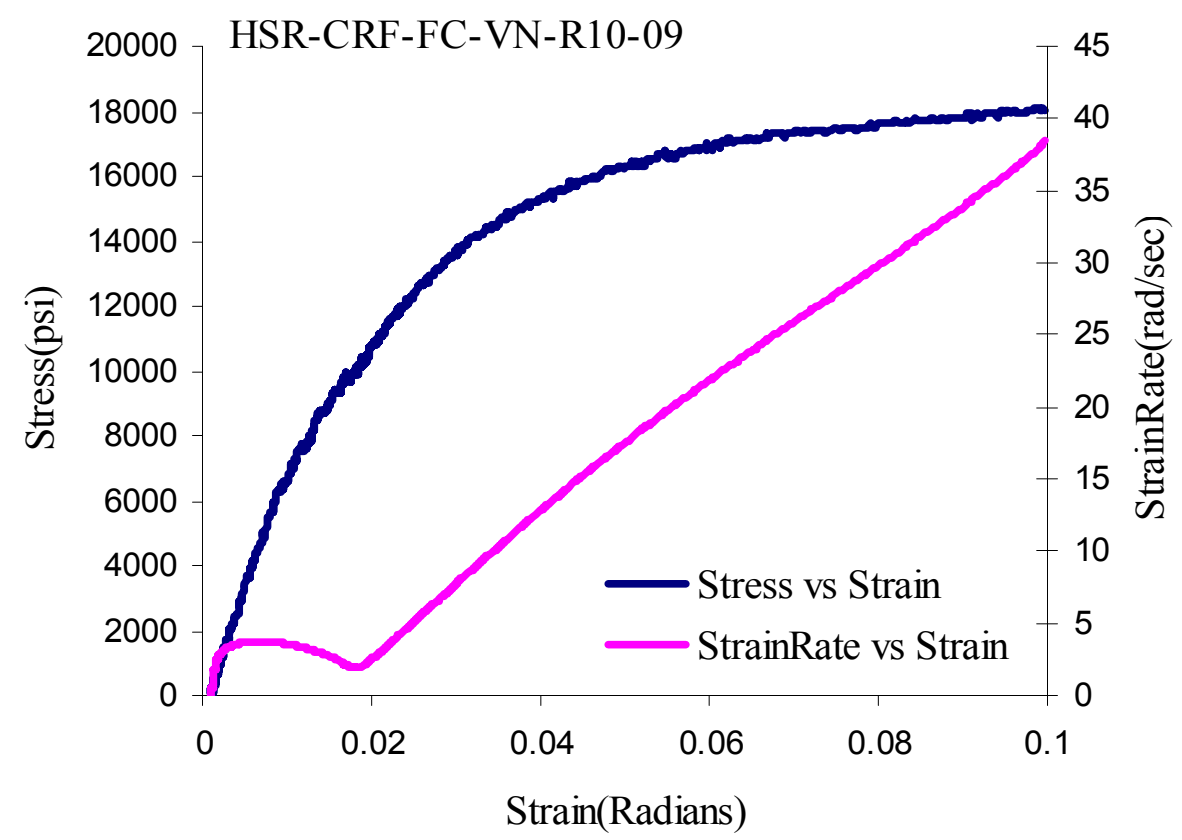

Figure 67. Stress-Strain and Strain Rate-Strain behavior of Fibercote at $10 \mathrm{in} / \mathrm{s}$ stroke rate 


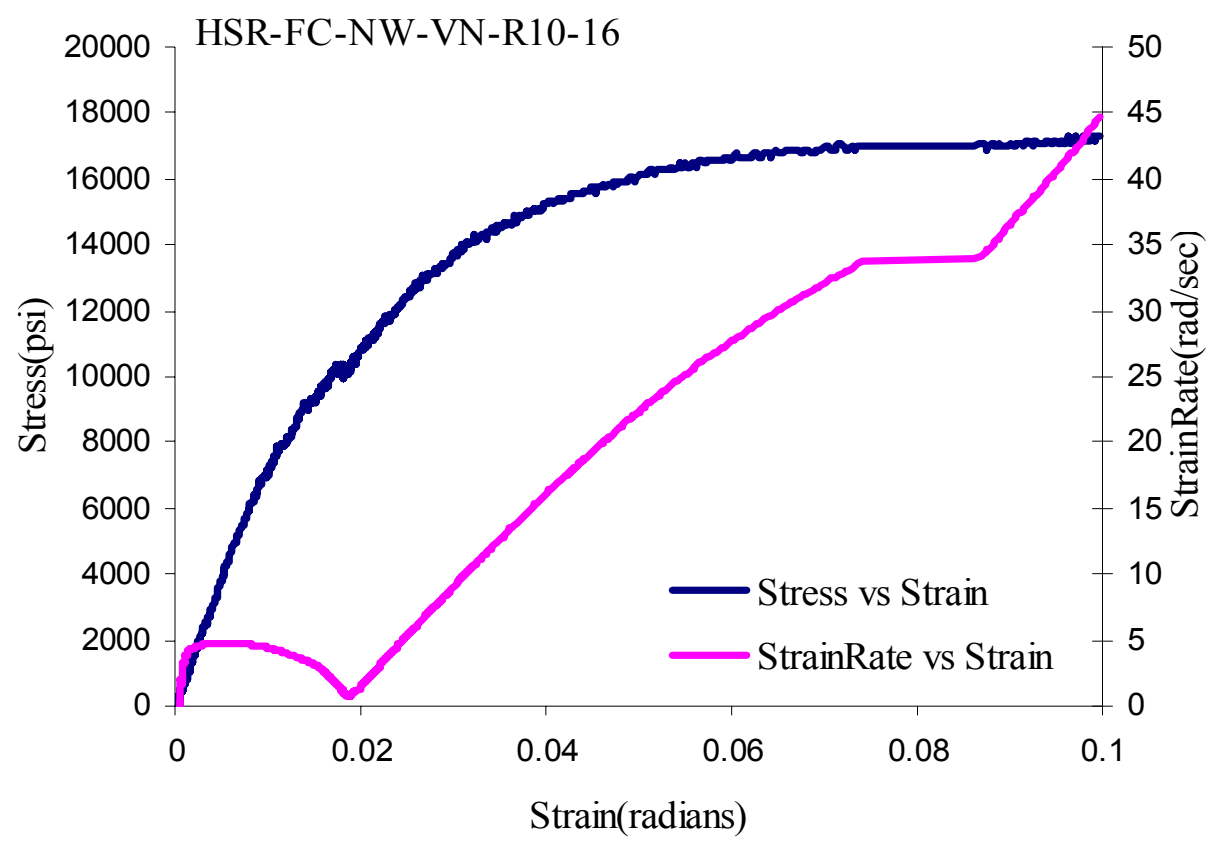

Figure 68. Stress-Strain and Strain Rate-Strain behavior of Fibercote at $10 \mathrm{in} / \mathrm{s}$ stroke rate

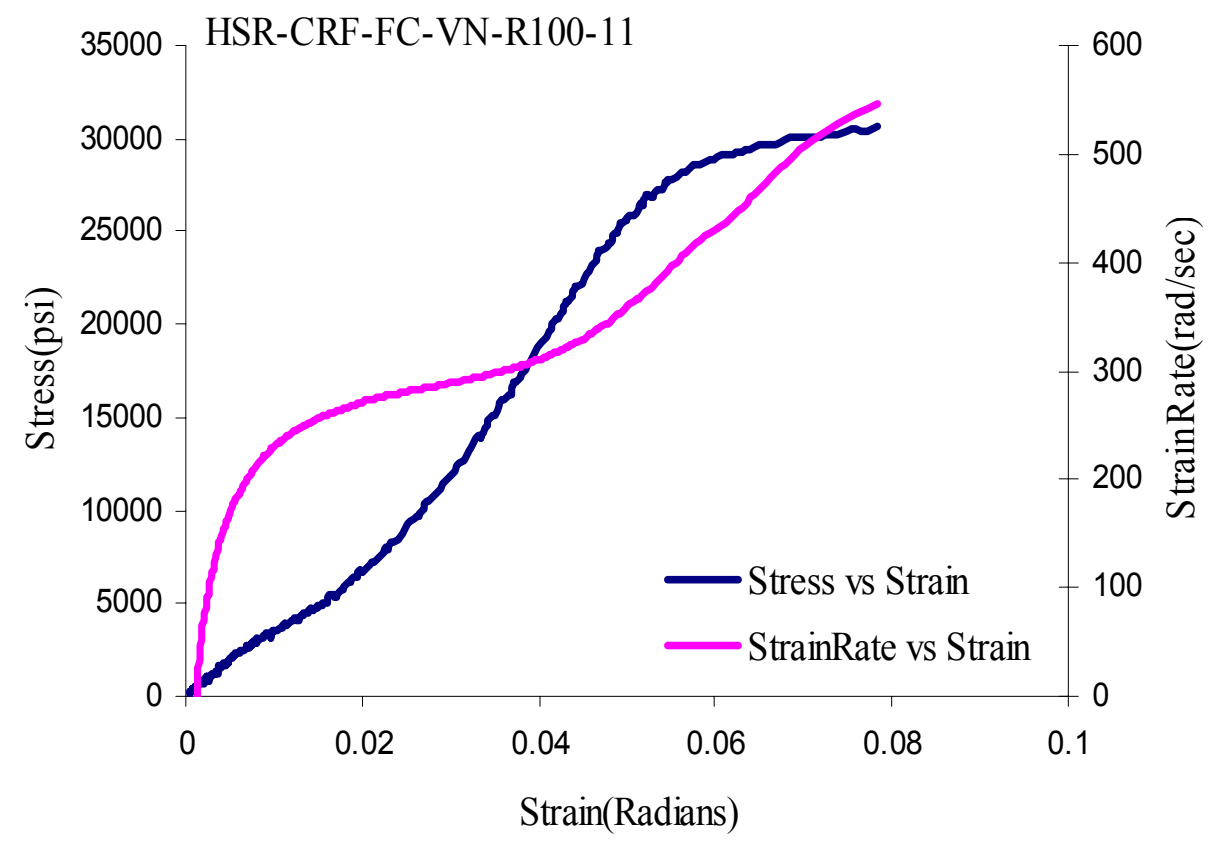

Figure 69. Stress-Strain and Strain Rate-Strain behavior of Fibercote at $100 \mathrm{in} / \mathrm{s}$ stroke rate 


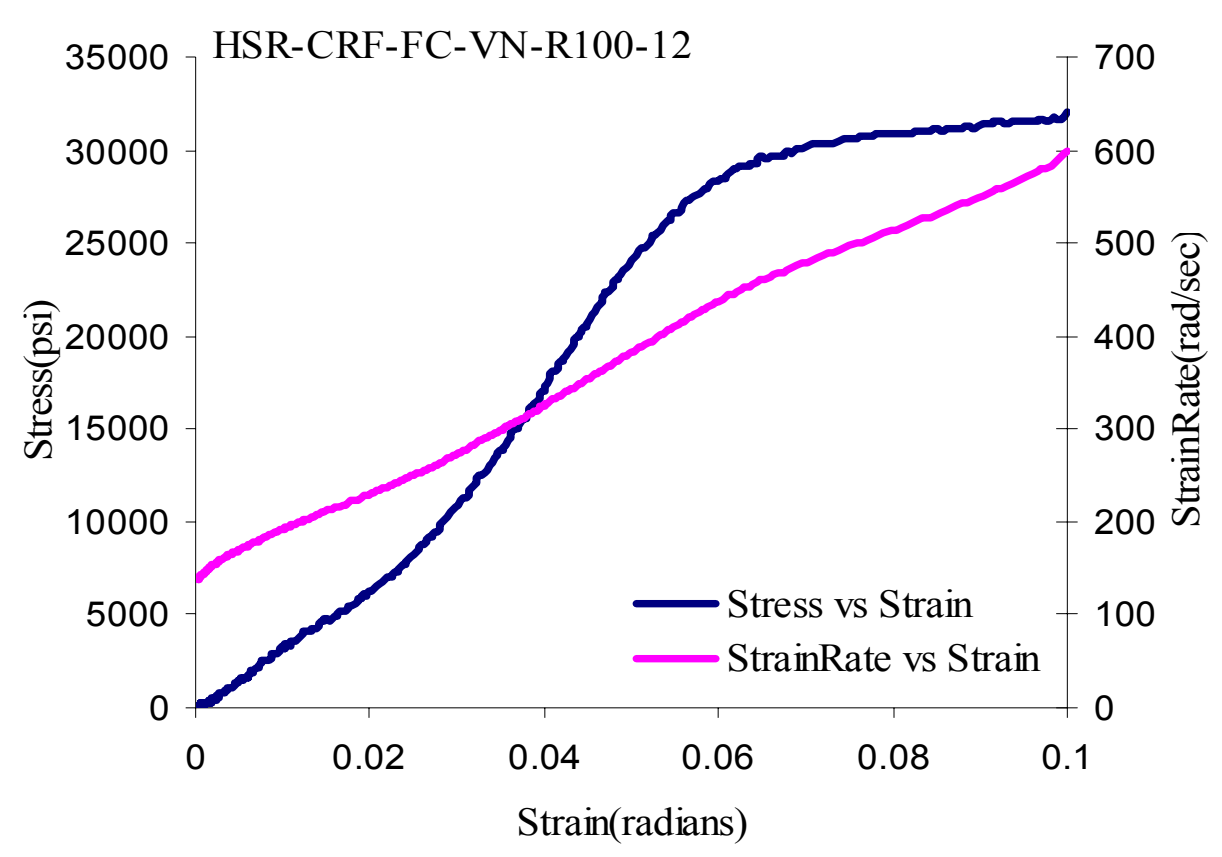

Figure 70. Stress-Strain and Strain Rate-Strain behavior of Fibercote at $100 \mathrm{in} / \mathrm{s}$ stroke rate

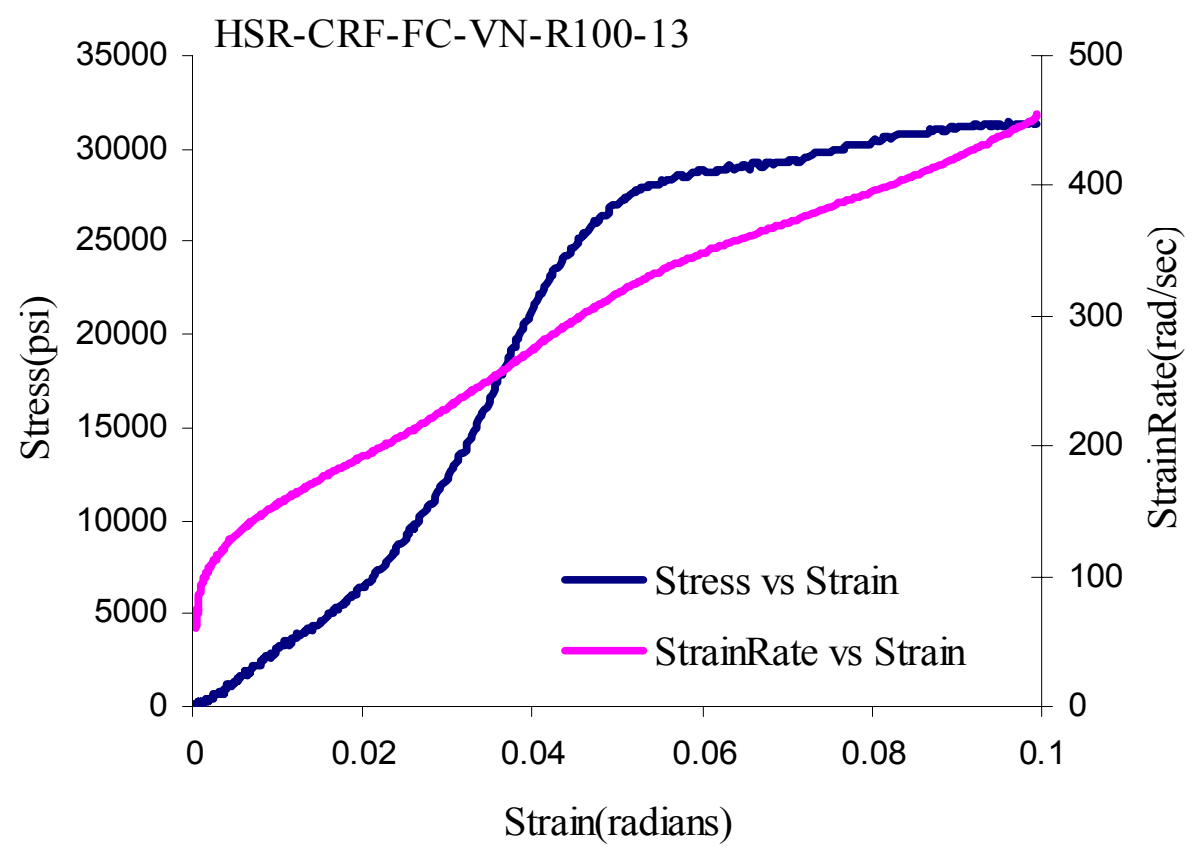

Figure 71. Stress-Strain and Strain Rate-Strain behavior of Fibercote at $100 \mathrm{in} / \mathrm{s}$ stroke rate 


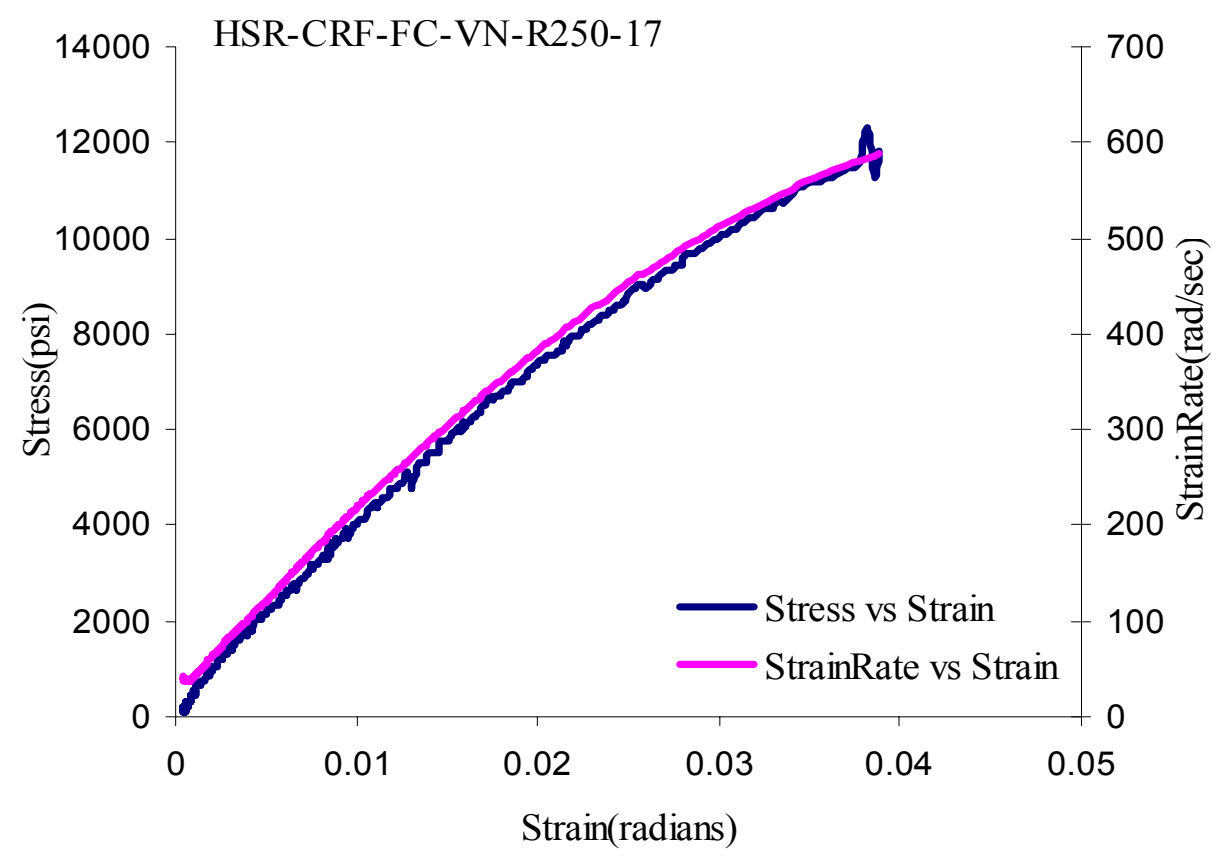

Figure 72. Stress-Strain and Strain Rate-Strain behavior of Fibercote at $250 \mathrm{in} / \mathrm{s}$ stroke rate

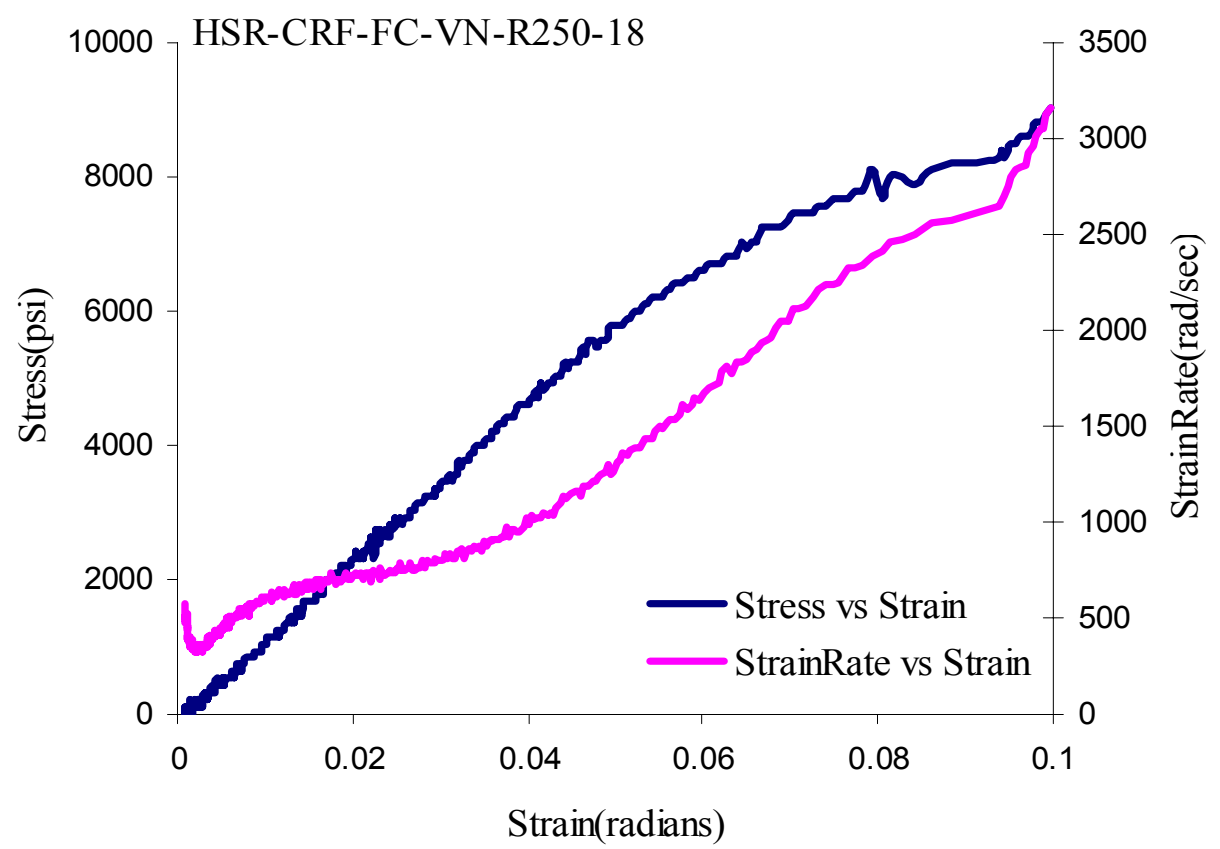

Figure 73. Stress-Strain and Strain Rate-Strain behavior of Fibercote at $250 \mathrm{in} / \mathrm{s}$ stroke rate 


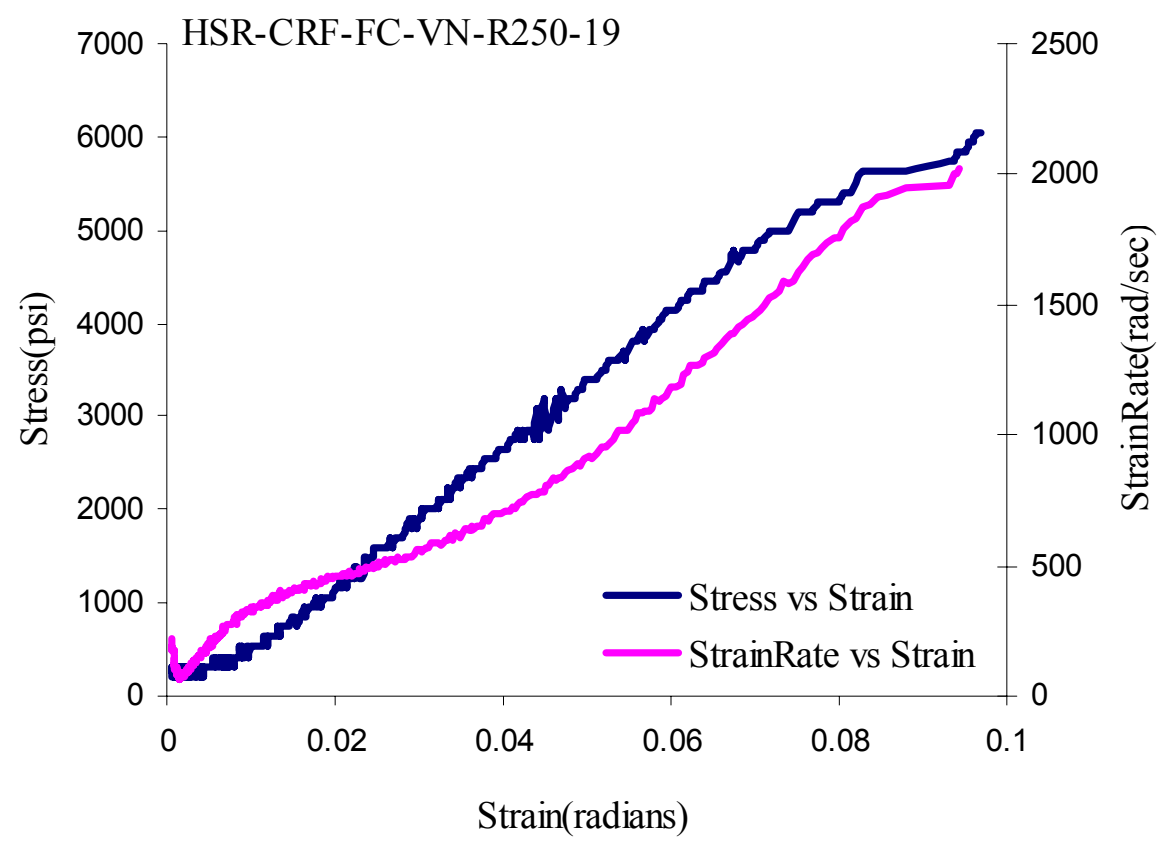

Figure 74. Stress-Strain and Strain Rate-Strain behavior of Fibercote at $250 \mathrm{in} / \mathrm{s}$ stroke rate

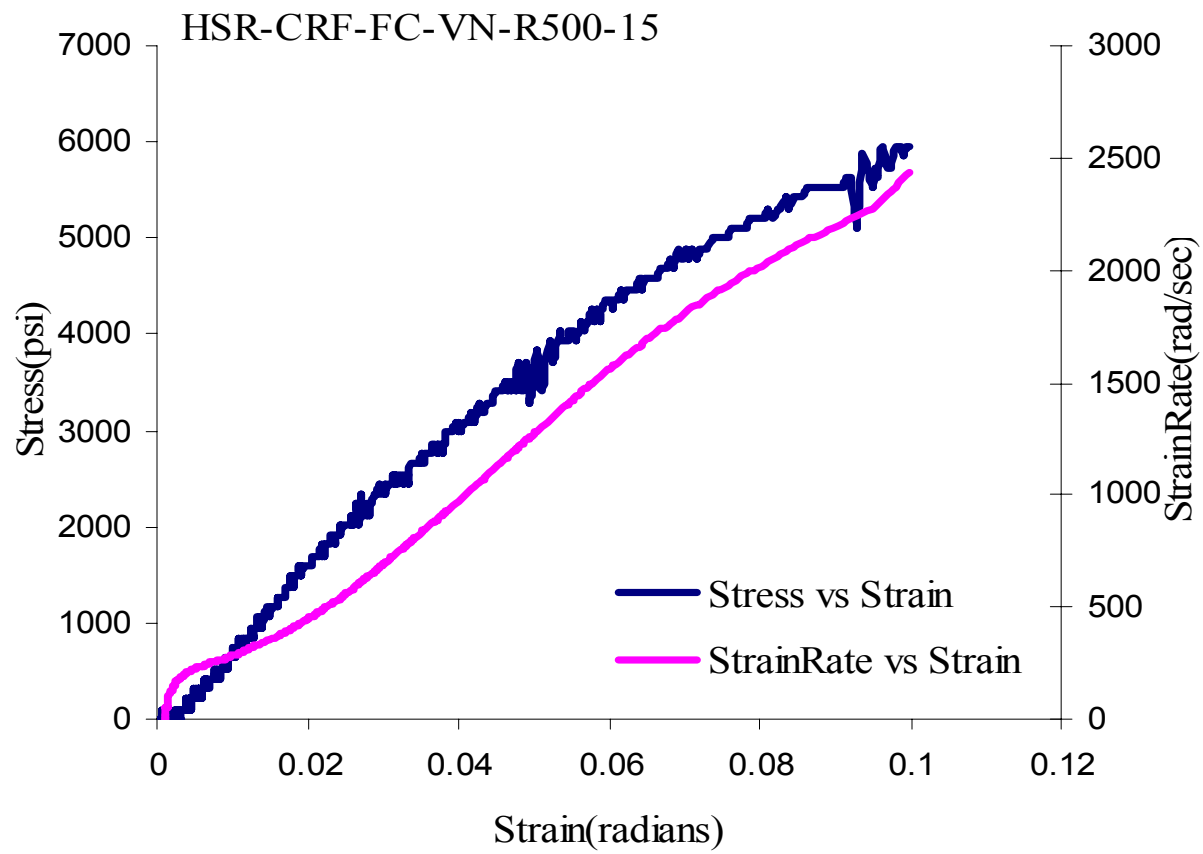

Figure 75. Stress-Strain and Strain Rate-Strain behavior of Fibercote at $500 \mathrm{in} / \mathrm{s}$ stroke rate 


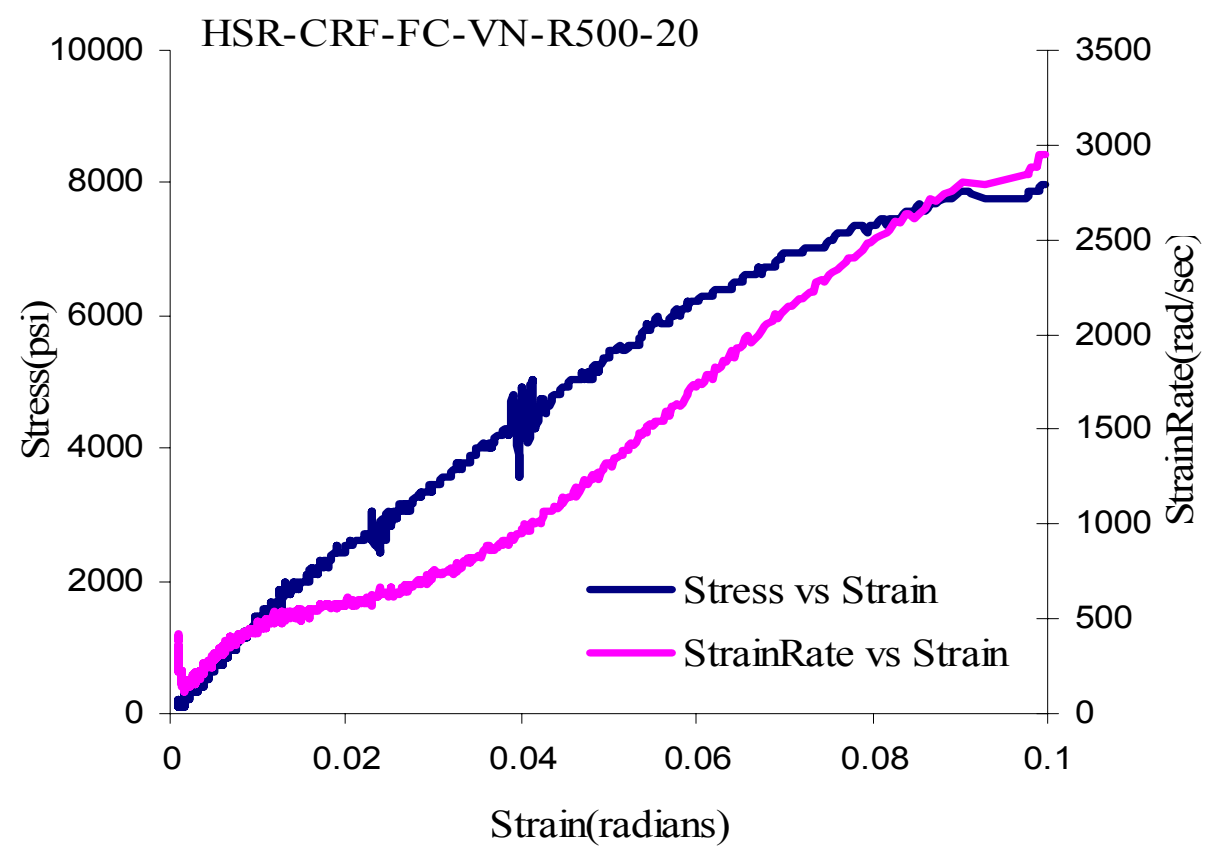

Figure 76. Stress-Strain and Strain Rate-Strain behavior of Fibercote at $500 \mathrm{in} / \mathrm{s}$ stroke rate

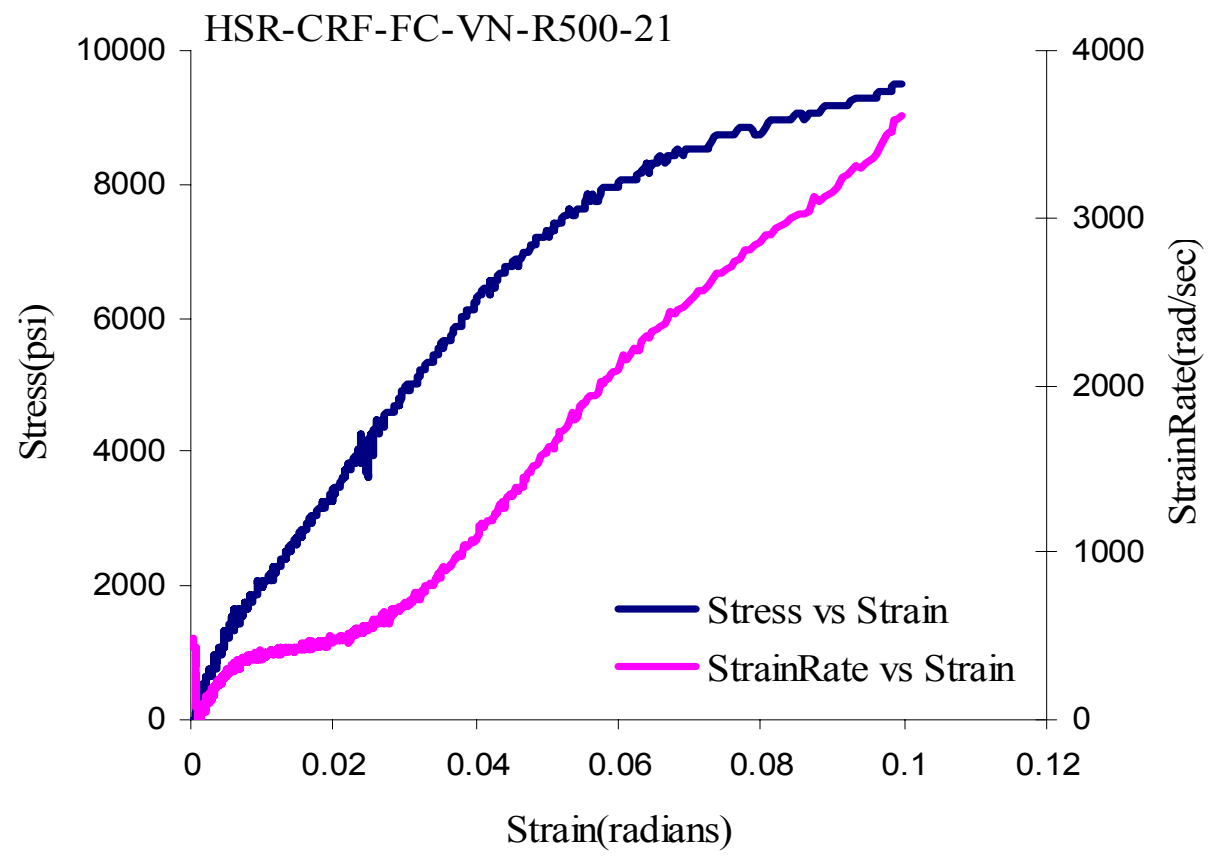

Figure 77. Stress-Strain and Strain Rate-Strain behavior of Fibercote at $500 \mathrm{in} / \mathrm{s}$ stroke rate 
Failure Patterns of Fibercote Specimens at different Stroke Rates

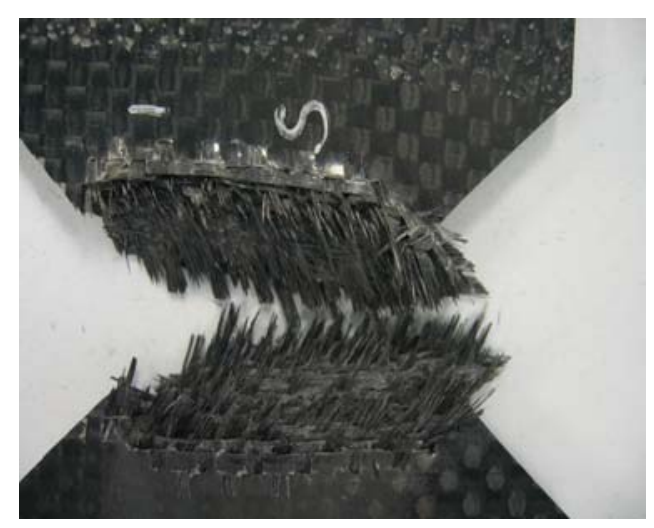

Figure 78. Failure mode of Fibercote material at $0.00083 \mathrm{in} / \mathrm{sec}$

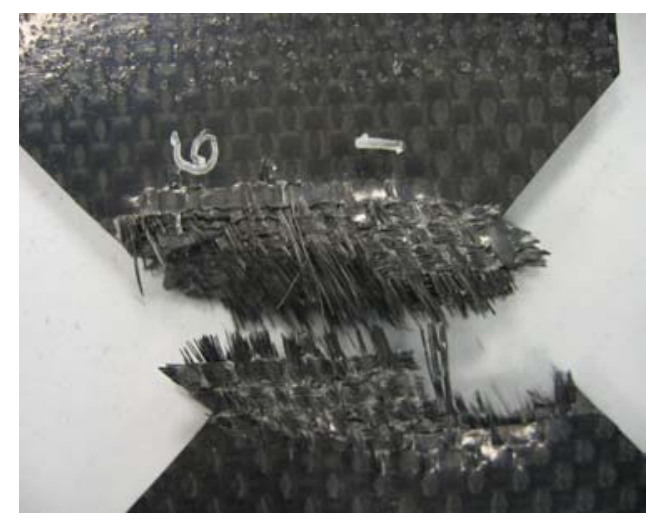

Figure 79. Failure mode of Fibercote material at $1 \mathrm{in} / \mathrm{sec}$

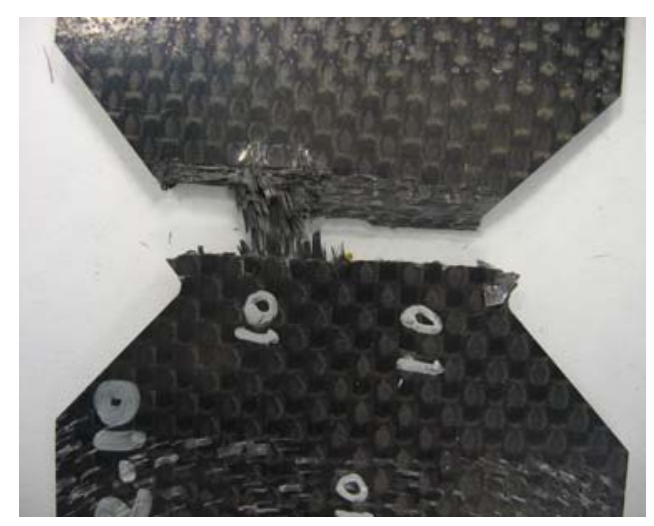

Figure 80 . Failure mode of Fibercote material at $10 \mathrm{in} / \mathrm{sec}$ 


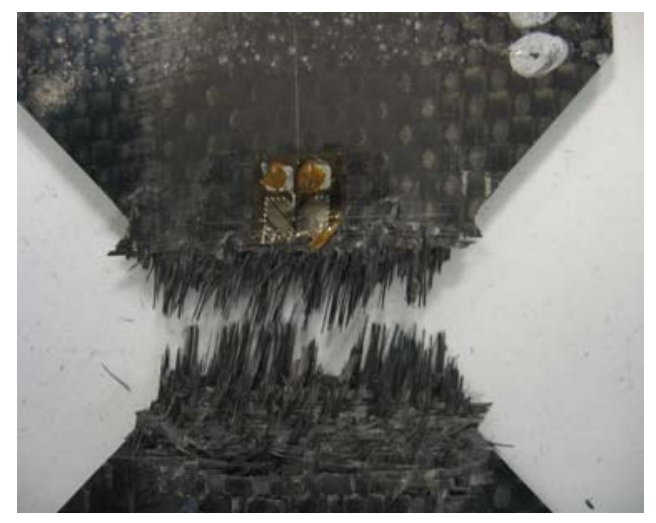

Figure 81. Failure mode of Fibercote material at $100 \mathrm{in} / \mathrm{sec}$

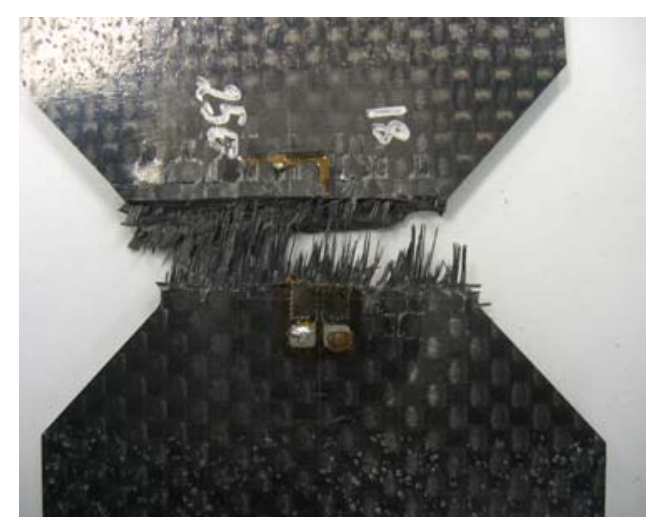

Figure 82. Failure mode of Fibercote material at $250 \mathrm{in} / \mathrm{sec}$

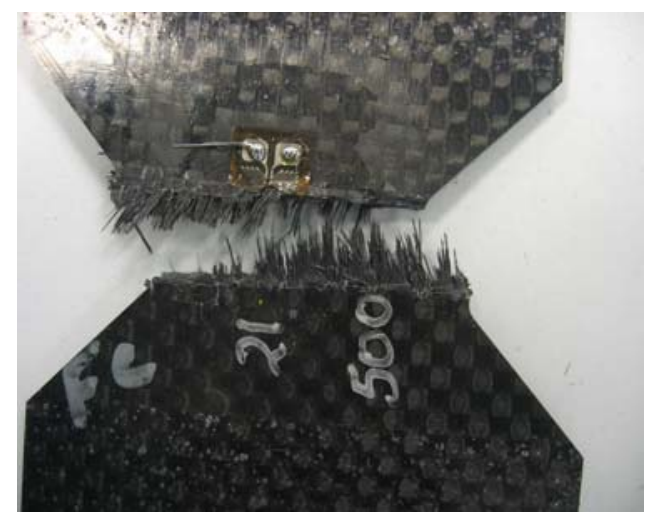

Figure 83. Failure mode of Fibercote material at $500 \mathrm{in} / \mathrm{sec}$ 
Table 9

Summary of test data for Fibercote material

\begin{tabular}{|c|c|c|c|c|c|}
\hline Specimen \# & $\begin{array}{l}\text { Average } \\
\text { Thickness } \\
\text { (in) }\end{array}$ & $\begin{array}{c}\text { Average Notch } \\
\text { to Notch } \\
\text { Width (in) }\end{array}$ & $\begin{array}{c}\text { Failure } \\
\text { Stress } \\
\left(\mathrm{lbf} / \mathrm{in}^{2}\right) \\
\end{array}$ & $\begin{array}{l}\text { Max } \\
\text { Load } \\
\text { (lbs) }\end{array}$ & $\begin{array}{c}\text { Average } \\
\text { Strain Rate } \\
(\mathrm{rad} / \mathrm{sec}) \\
\end{array}$ \\
\hline HSR-CRF-FC-VN-static-01 & 0.097 & 1.220 & 22062.18 & 2615.87 & 0.00078 \\
\hline HSR-CRF-FC-VN-static-02 & 0.098 & 1.217 & 19045.53 & 2274.91 & 0.00074 \\
\hline HSR-CRF-FC-VN-static-14 & 0.097 & 1.220 & 18977.40 & 2239.33 & 0.00048 \\
\hline HSR-CRF-FC-VN-R1-04 & 0.096 & 1.221 & 21339.04 & 2488.28 & 0.99 \\
\hline HSR-CRF-FC-VN-R1-06 & 0.098 & 1.218 & 22743.04 & 2704.10 & 0.91 \\
\hline HSR-CRF-FC-VN-R1-07 & 0.099 & 1.220 & 22837.93 & 2754.88 & 1.02 \\
\hline HSR-CRF-FC-VN-R10-08 & 0.097 & 1.222 & 24989.30 & 2970.70 & 7.68 \\
\hline HSR-CRF-FC-VN-R10-09 & 0.098 & 1.222 & 24843.64 & 2970.70 & 7.53 \\
\hline HSR-CRF-FC-VN-R10-16 & 0.101 & 1.220 & 23298.90 & 2856.45 & 6.84 \\
\hline HSR-CRF-FC-VN-R100-11 & 0.098 & 1.220 & 39993.57 & 4798.83 & 254.90 \\
\hline HSR-CRF-FC-VN-R100-12 & 0.095 & 1.220 & 33018.57 & 3833.98 & 310.15 \\
\hline HSR-CRF-FC-VN-R100-13 & 0.098 & 1.220 & 36753.80 & 4405.27 & 241.91 \\
\hline HSR-CRF-FC-VN-R250-17 & 0.098 & 1.221 & 29533.37 & 3529.30 & 203.42 \\
\hline HSR-CRF-FC-VN-R250-18 & 0.099 & 1.221 & 32477.16 & 3922.85 & 880.92 \\
\hline HSR-CRF-FC-VN-R250-19 & 0.098 & 1.221 & 42662.26 & 5103.52 & 551.55 \\
\hline HSR-CRF-FC-VN-R500-15 & 0.098 & 1.221 & 25837.16 & 3084.96 & 574.96 \\
\hline HSR-CRF-FC-VN-R500-20 & 0.099 & 1.221 & 25310.81 & 3059.57 & 708.09 \\
\hline HSR-CRF-FC-VN-R500-21 & 0.095 & 1.221 & 29151.75 & 3389.65 & 654.82 \\
\hline
\end{tabular}




\section{Stress-Strain and Strain Rate-Strain behavior of Newport PWCF at different stroke rates}

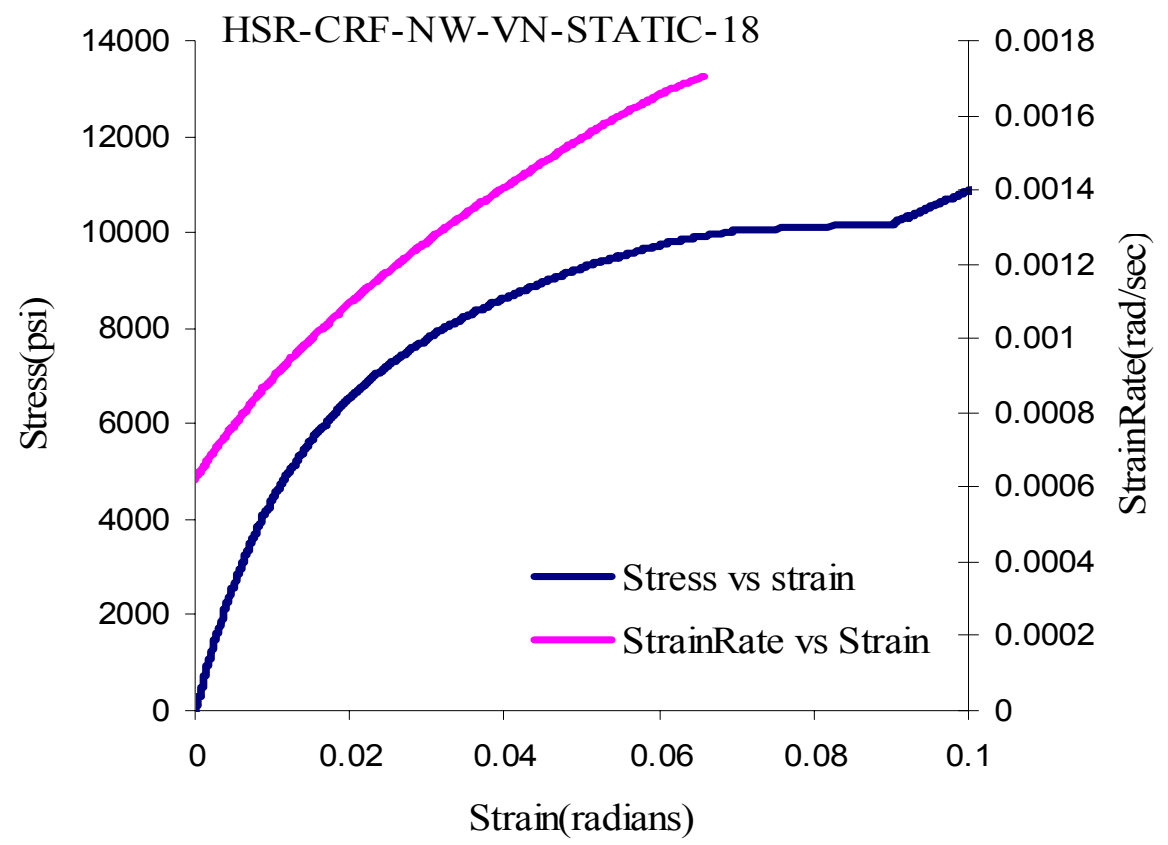

Figure 84. Stress-Strain and Strain Rate-Strain behavior of Newport PWCF at $0.00083 \mathrm{in} / \mathrm{s}$ stroke rate

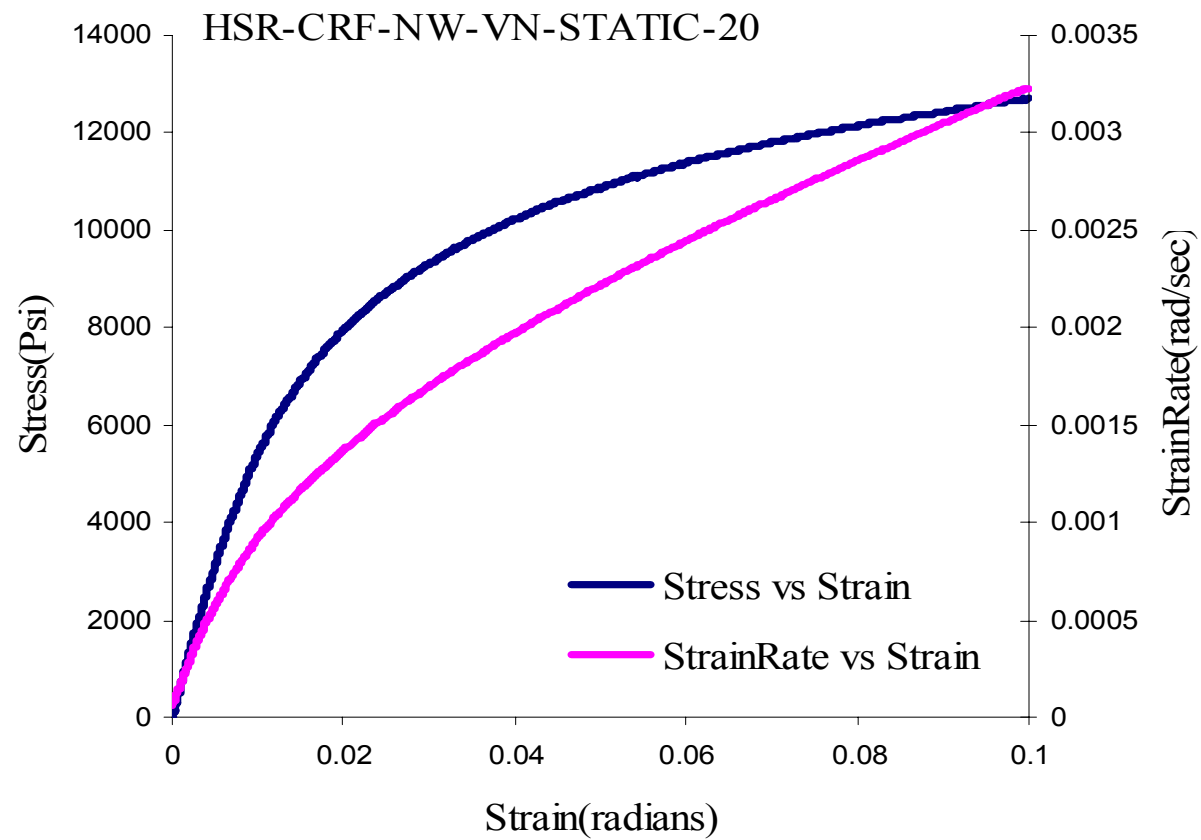

Figure 85. Stress-Strain and Strain Rate-Strain behavior of Newport PWCF at $0.00083 \mathrm{in} / \mathrm{s}$ stroke rate 


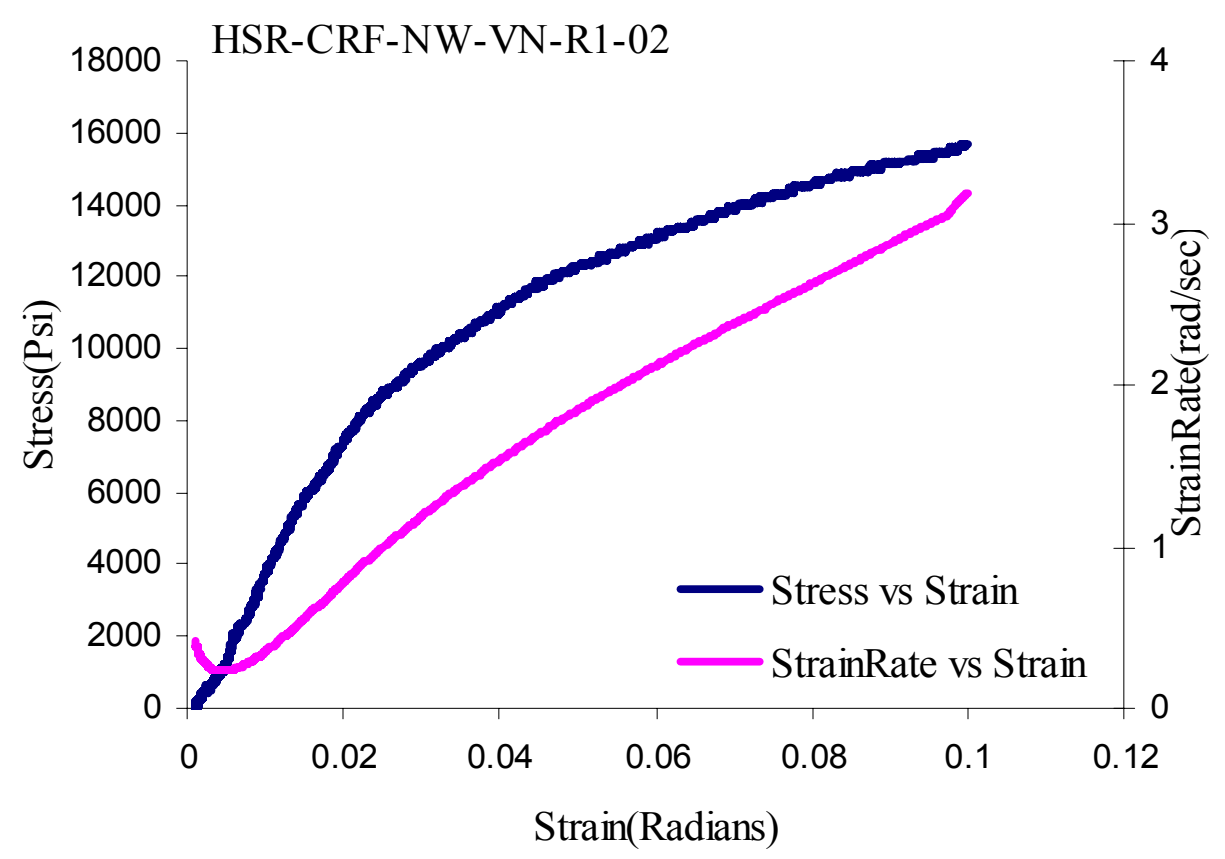

Figure 86. Stress-Strain and Strain Rate-Strain behavior of Newport PWCF at $1 \mathrm{in} / \mathrm{s}$ stroke rate

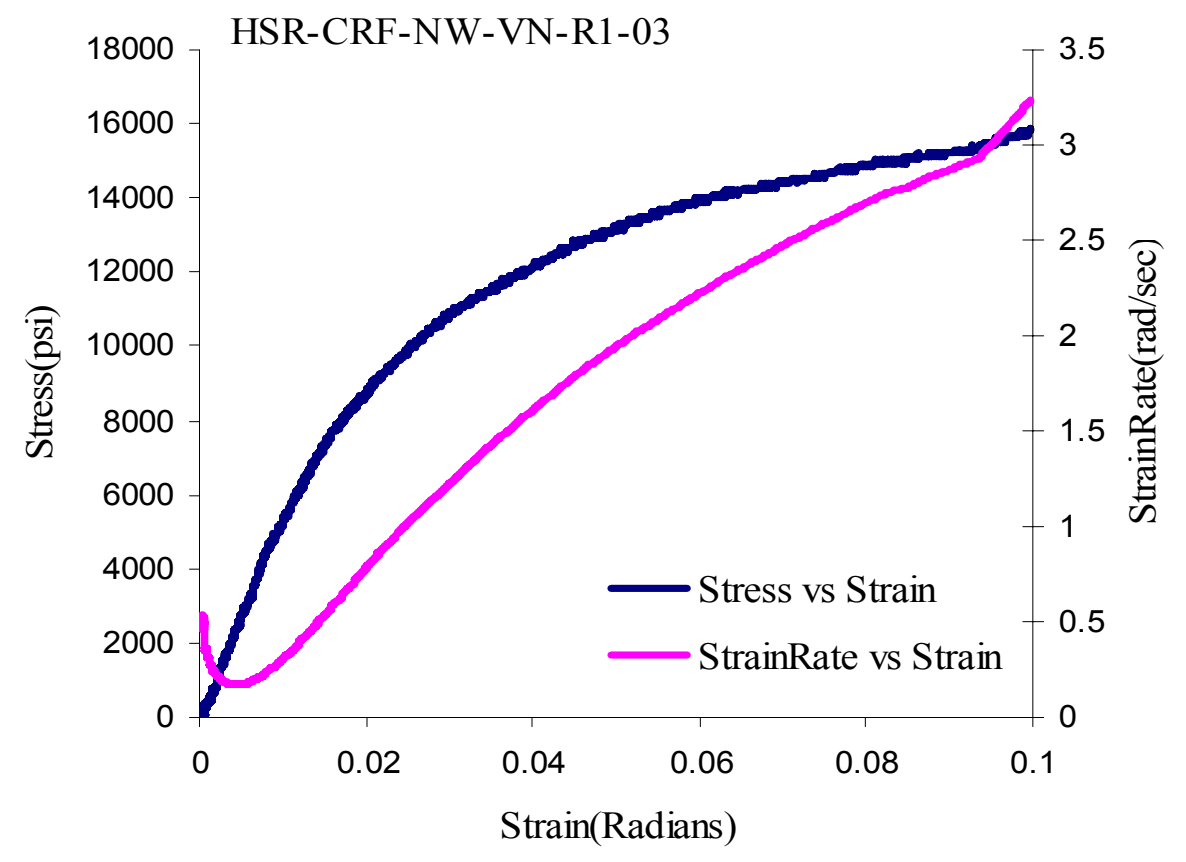

Figure 87. Stress-Strain and Strain Rate-Strain behavior of Newport PWCF at $1 \mathrm{in} / \mathrm{s}$ stroke rate 


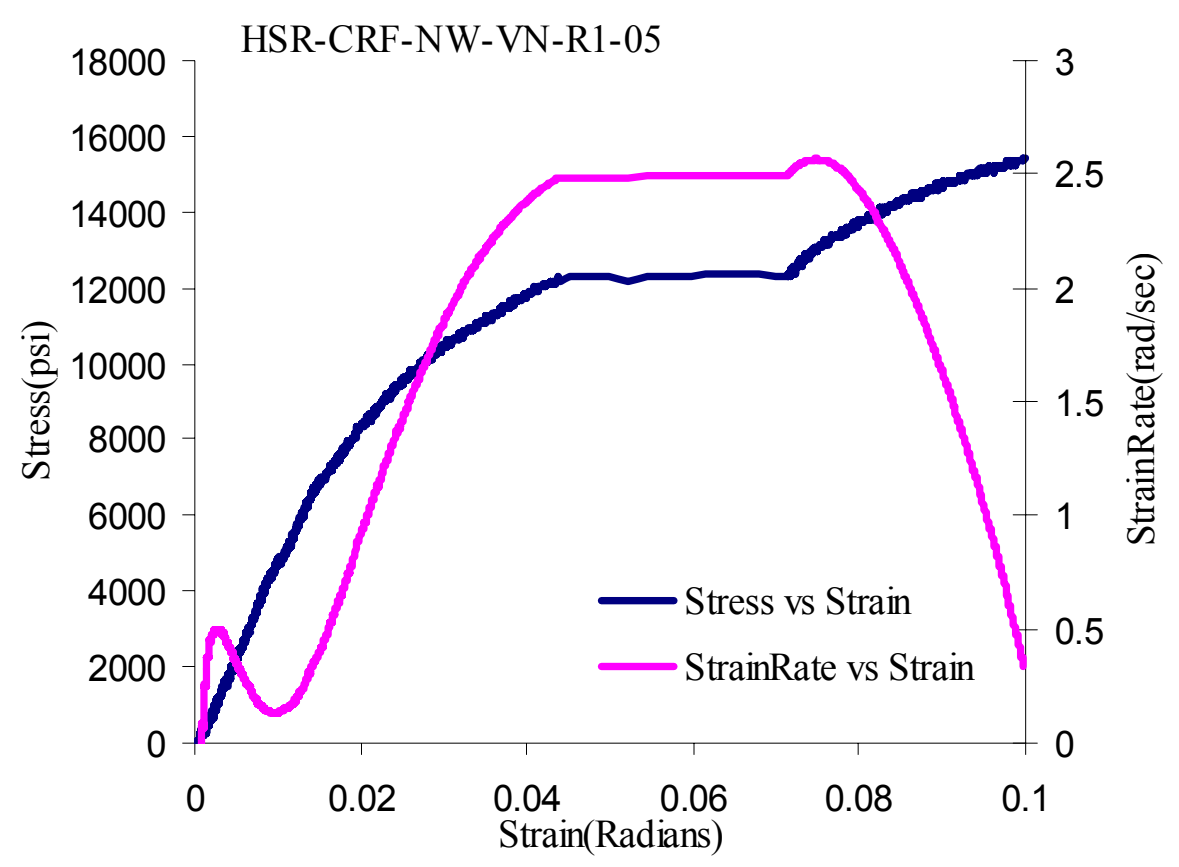

Figure 88. Stress-Strain and Strain Rate-Strain behavior of Newport PWCF at $1 \mathrm{in} / \mathrm{s}$ stroke rate

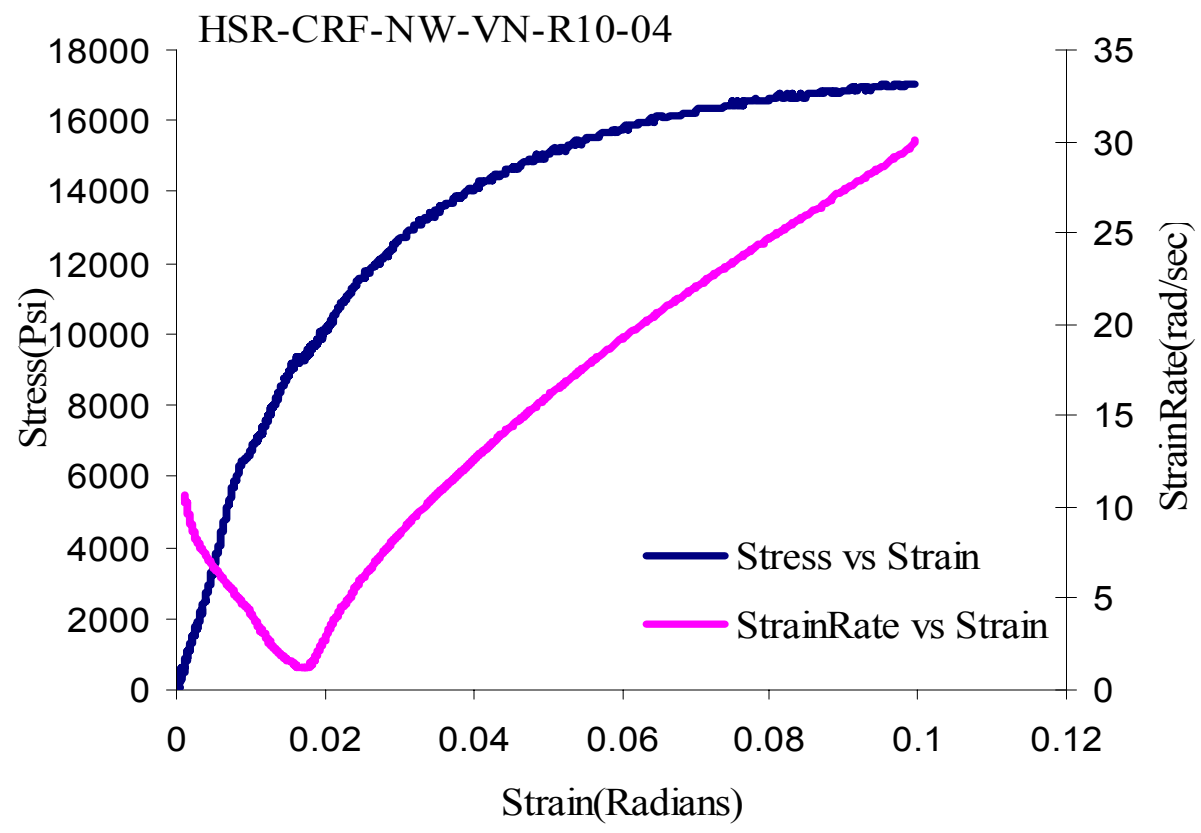

Figure 89. Stress-Strain and Strain Rate-Strain behavior of Newport PWCF at $10 \mathrm{in} / \mathrm{s}$ stroke rate 


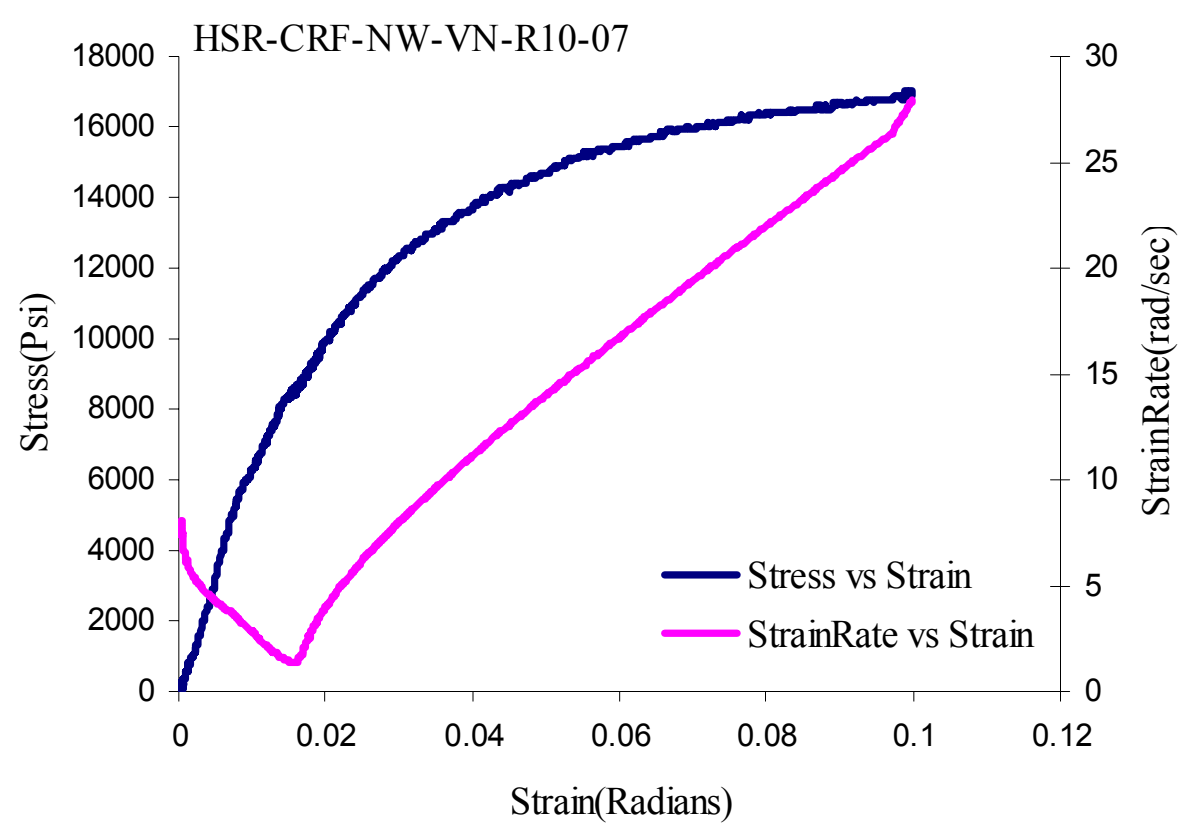

Figure 90. Stress-Strain and Strain Rate-Strain behavior of Newport PWCF at $10 \mathrm{in} / \mathrm{s}$ stroke Rate

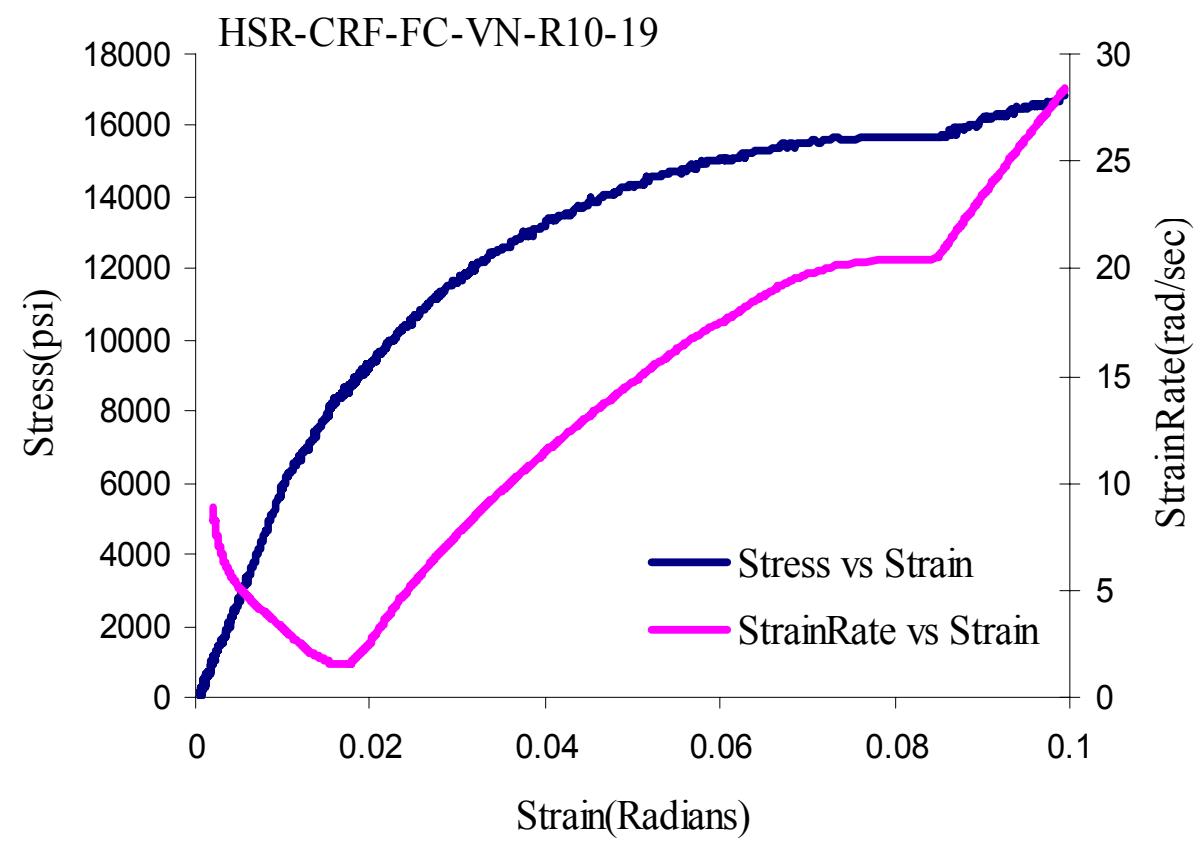

Figure 91. Stress-Strain and Strain Rate-Strain behavior of Newport PWCF at $10 \mathrm{in} / \mathrm{s}$ stroke rate 


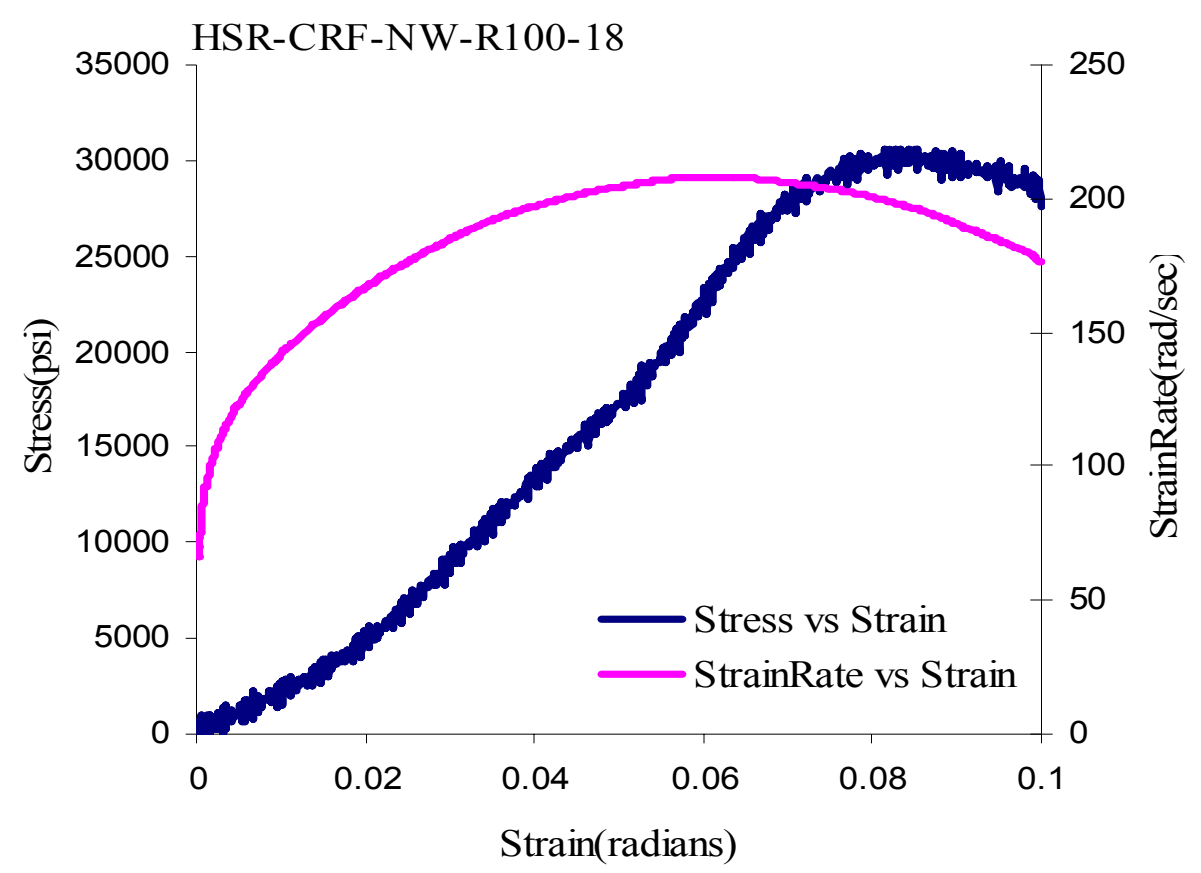

Figure 92. Stress-Strain and Strain Rate-Strain behavior of Newport PWCF at $100 \mathrm{in} / \mathrm{s}$ stroke rate

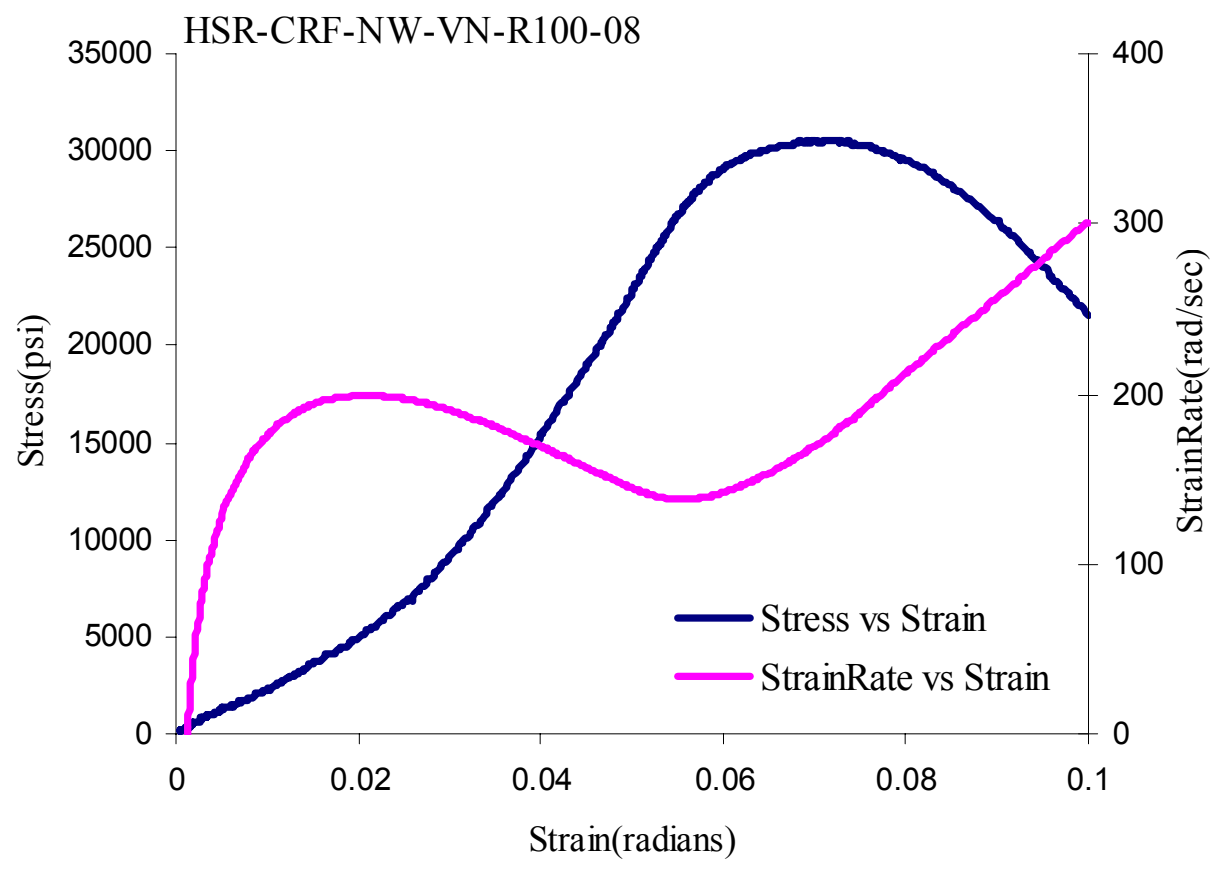

Figure 93. Stress-Strain and Strain Rate-Strain behavior of Newport PWCF at $100 \mathrm{in} / \mathrm{s}$ stroke rate 


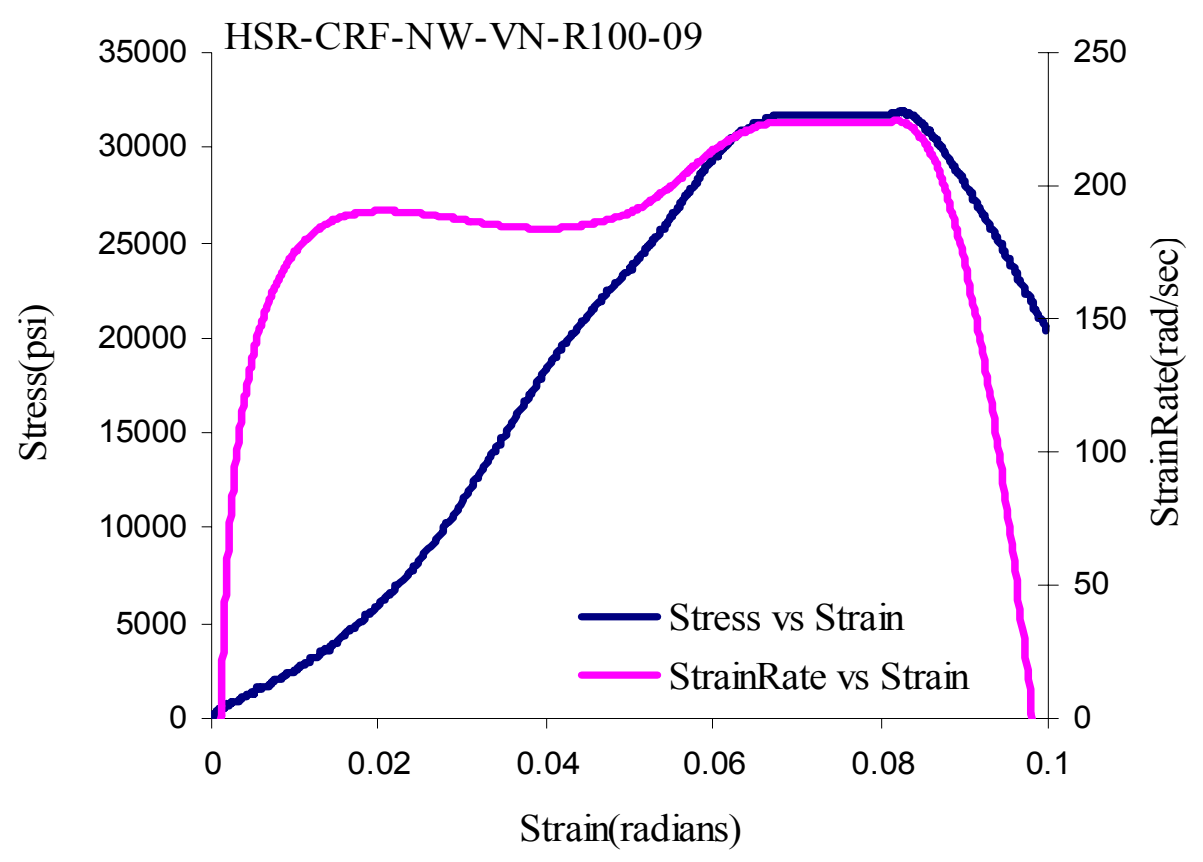

Figure 94. Stress-Strain and Strain Rate-Strain behavior of Newport PWCF at $100 \mathrm{in} / \mathrm{s}$ stroke rate

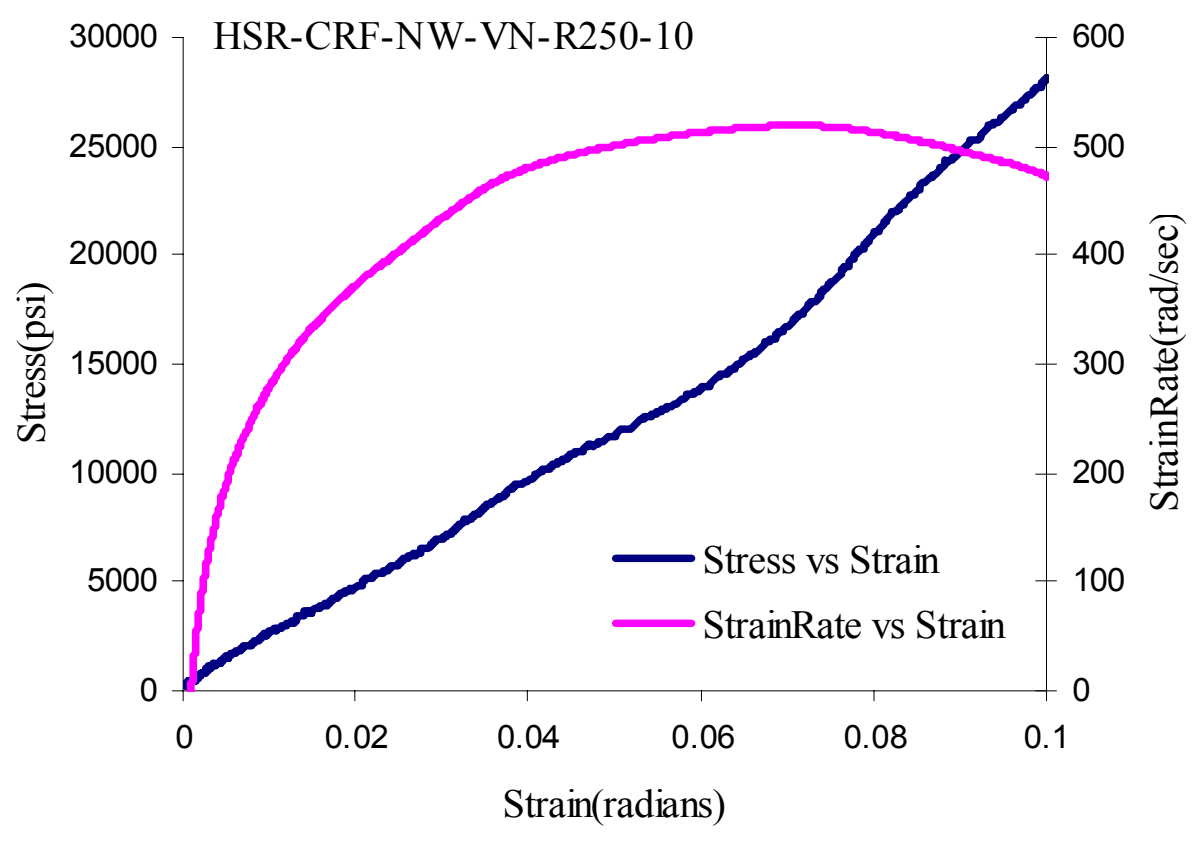

Figure 95. Stress-Strain and Strain Rate-Strain behavior of Newport PWCF at $250 \mathrm{in} / \mathrm{s}$ stroke rate 


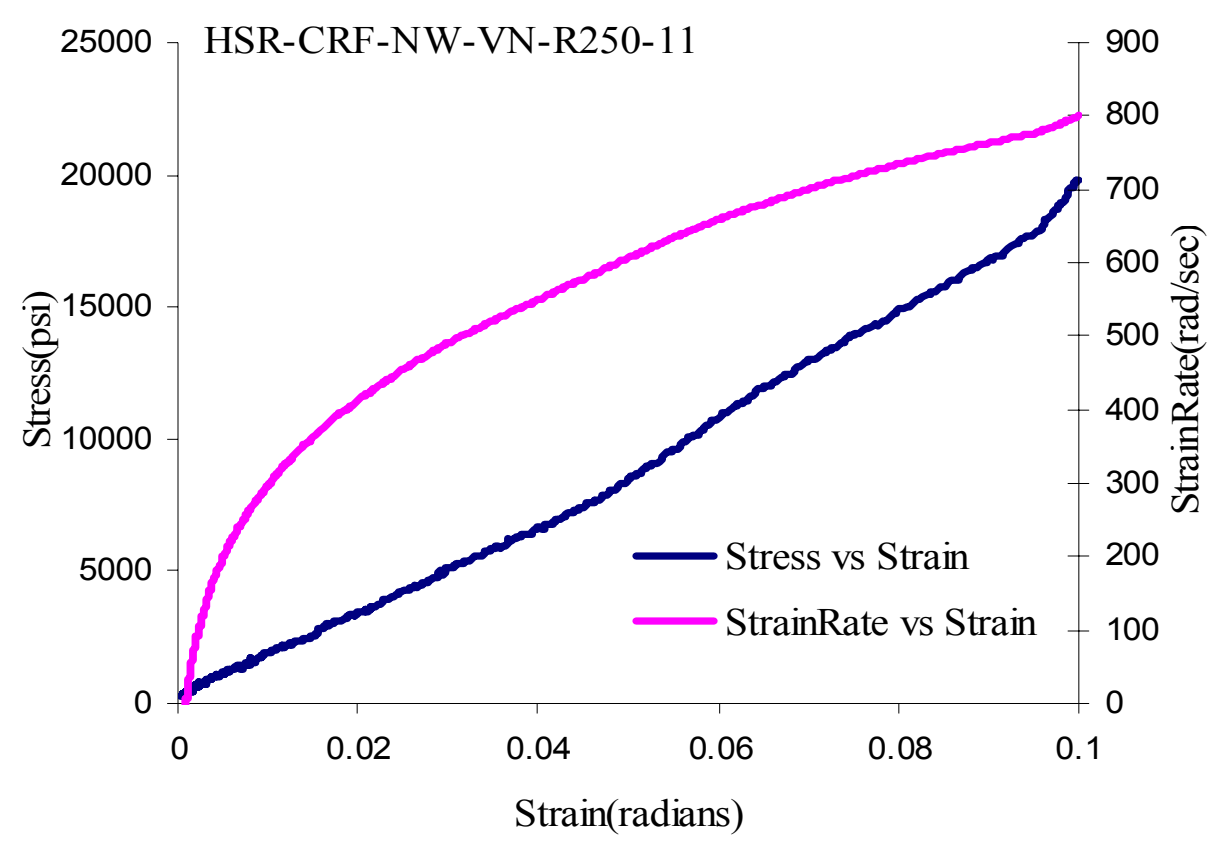

Figure 96. Stress-Strain and Strain Rate-Strain behavior of Newport PWCF at $250 \mathrm{in} / \mathrm{s}$ stroke rate

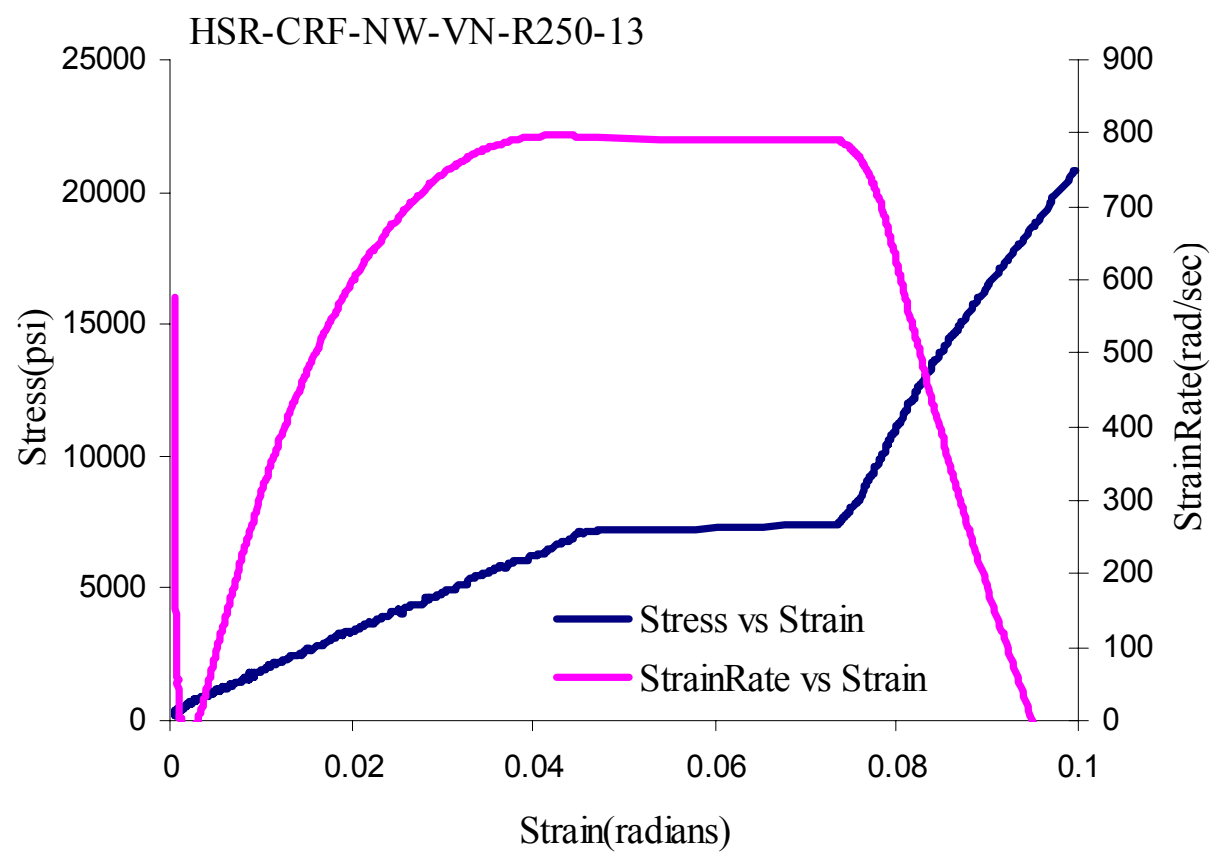

Figure 97. Stress-Strain and Strain Rate-Strain behavior of Newport PWCF at $250 \mathrm{in} / \mathrm{s}$ stroke rate 


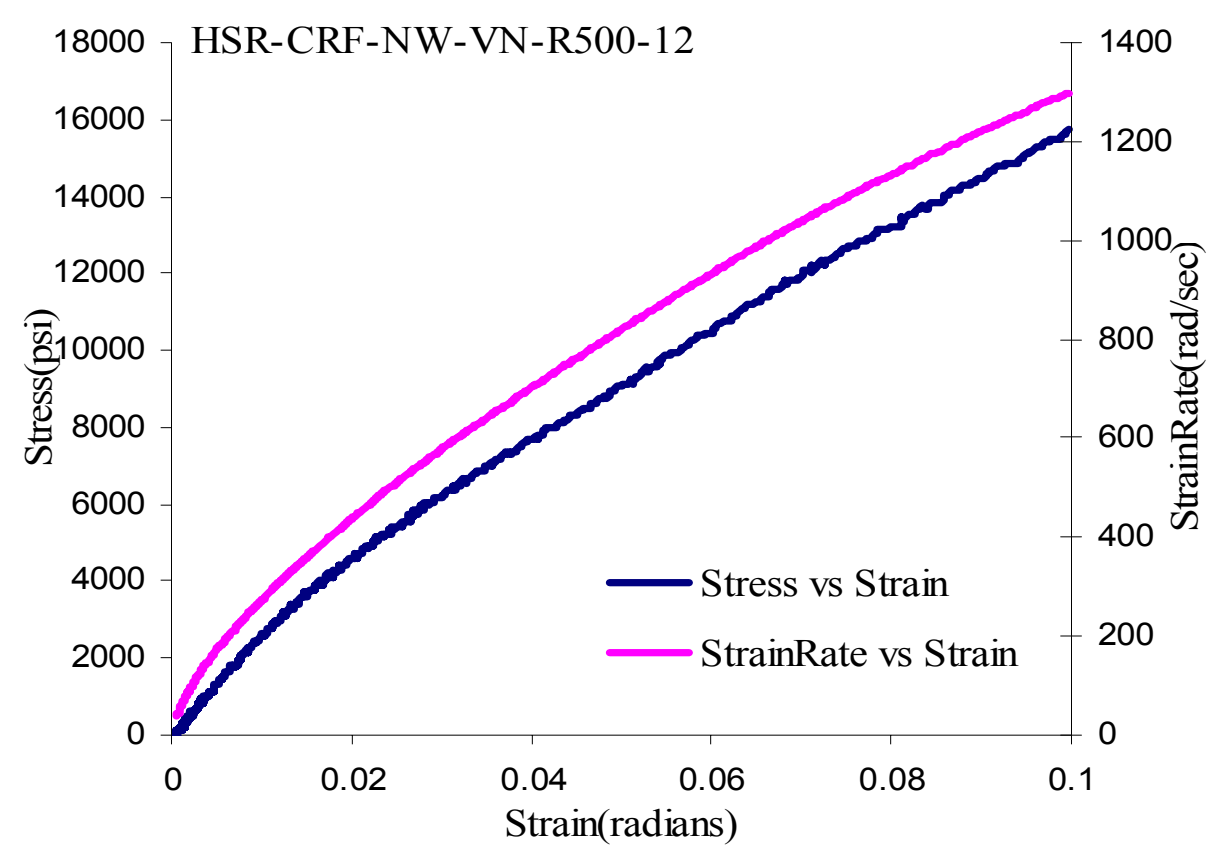

Figure 98. Stress-Strain and Strain Rate-Strain behavior of Newport PWCF at $500 \mathrm{in} / \mathrm{s}$ stroke rate

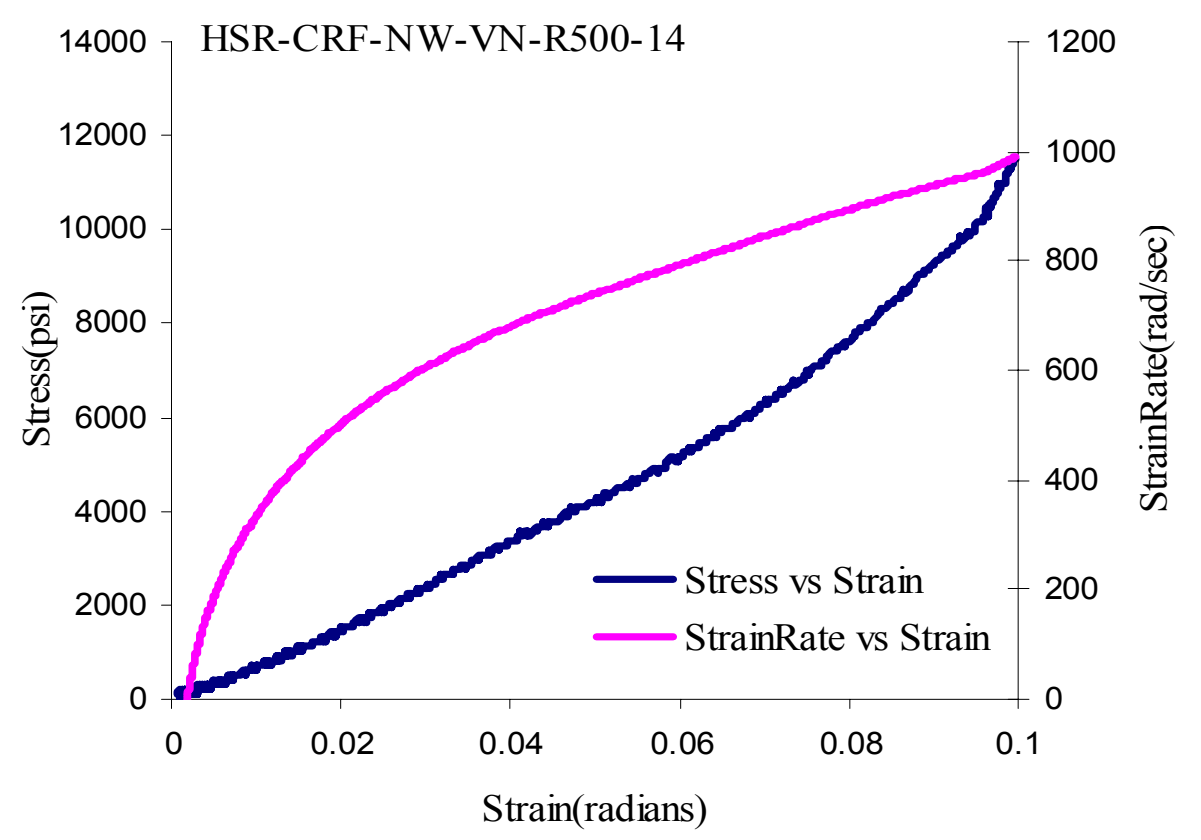

Figure 99. Stress-Strain and Strain Rate-Strain behavior of Newport PWCF at $500 \mathrm{in} / \mathrm{s}$ stroke rate 


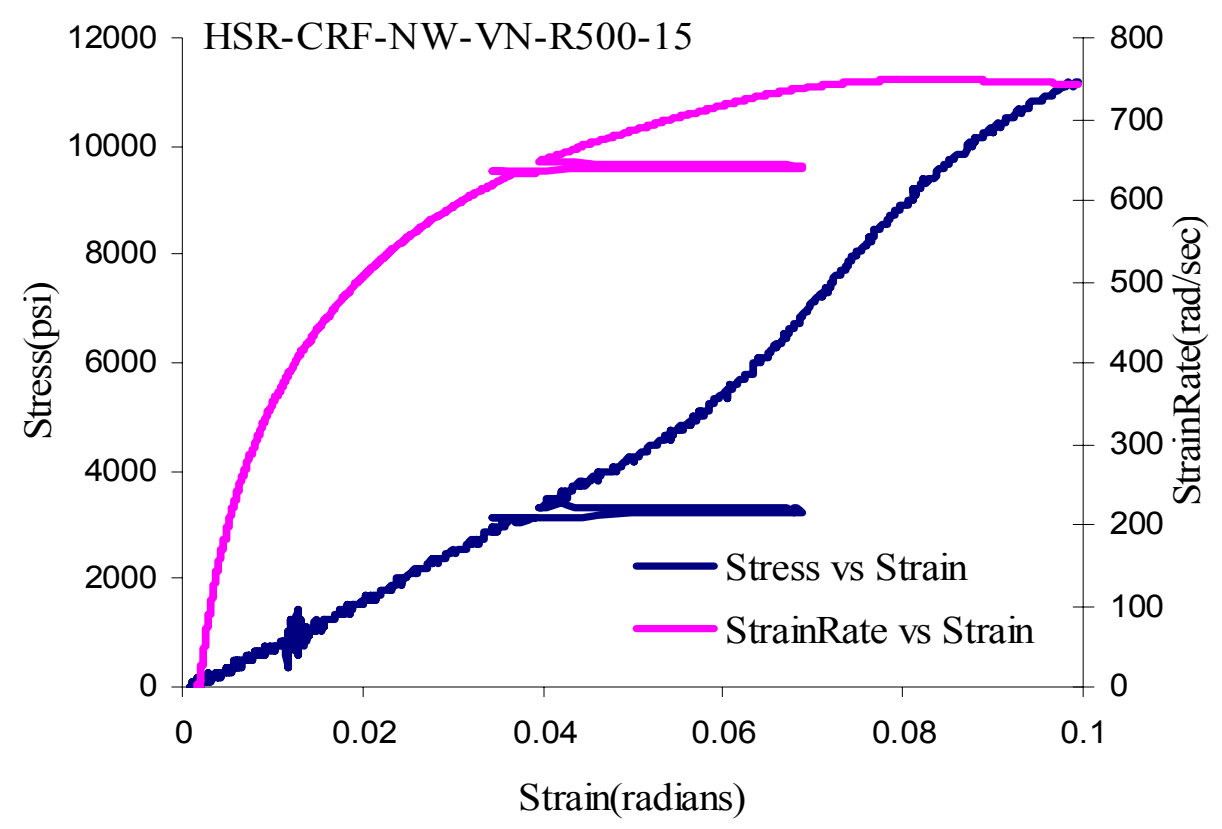

Figure 100. Stress-Strain and Strain Rate-Strain behavior of Newport PWCF at 500 in/s stroke rate

\section{Failure Patterns of Newport PWCF Specimens at different Stroke Rates}

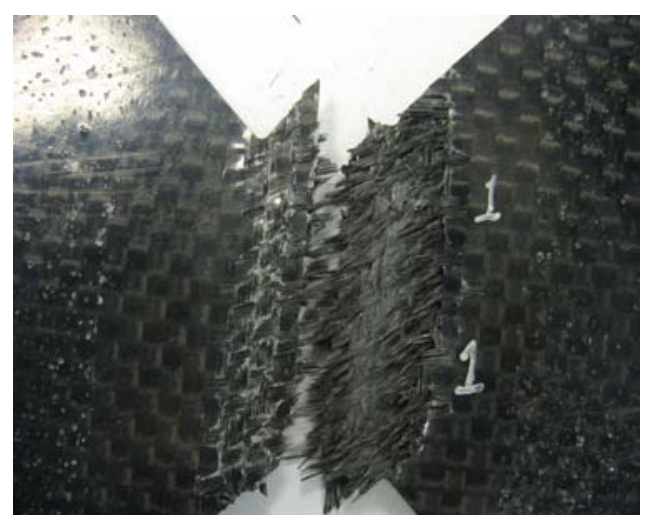

Figure 101. Failure mode of Newport PWCF material at $0.00083 \mathrm{in} / \mathrm{sec}$ 


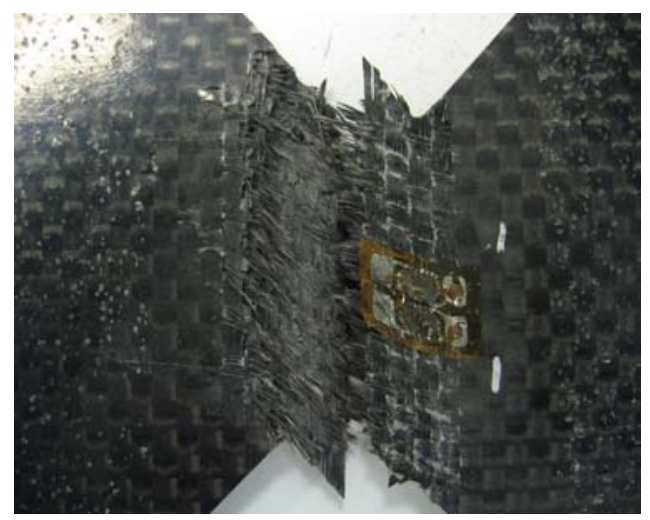

Figure 102. Failure mode of Newport PWCF material at $1 \mathrm{in} / \mathrm{sec}$

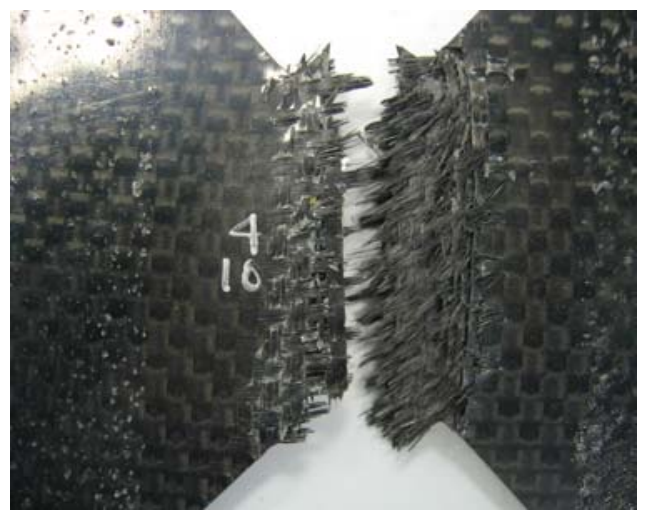

Figure 103. Failure mode of Newport PWCF material at $10 \mathrm{in} / \mathrm{sec}$

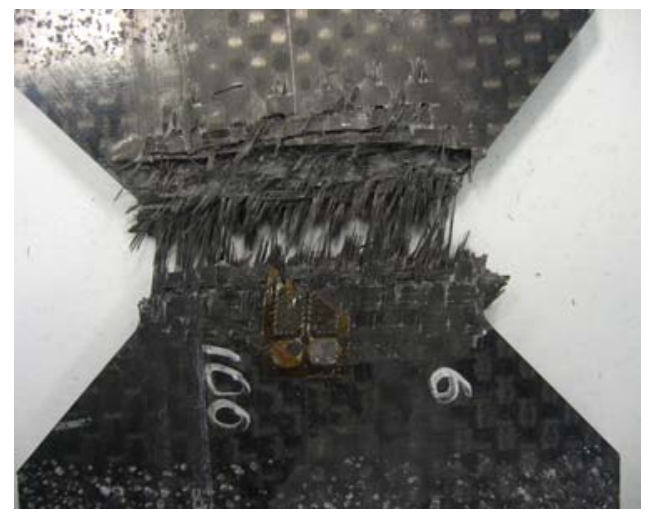

Figure 104. Failure mode of Newport PWCF material at $100 \mathrm{in} / \mathrm{sec}$ 


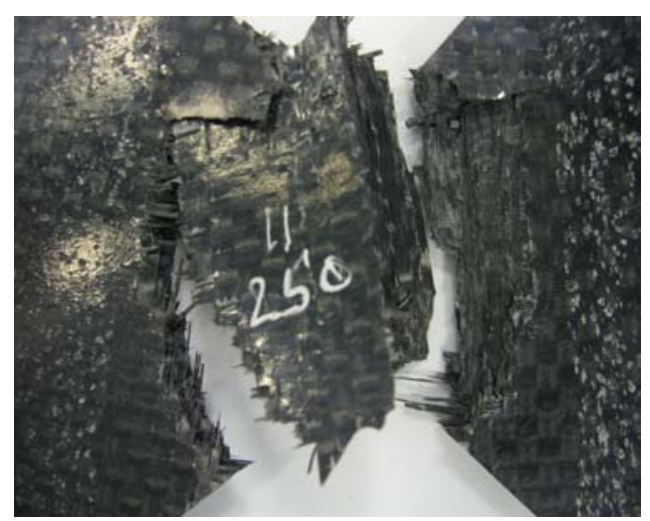

Figure 105. Failure mode of Newport PWCF material at $250 \mathrm{in} / \mathrm{sec}$

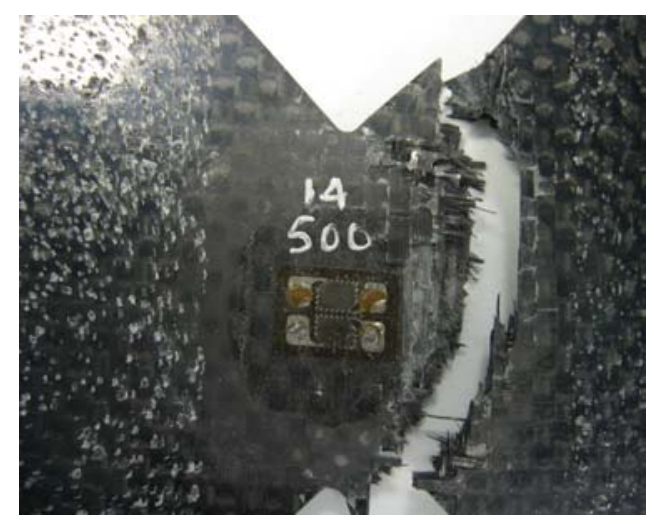

Figure 106. Failure mode of Newport PWCF material at $500 \mathrm{in} / \mathrm{sec}$ 
Table 10

Summary of test data for Newport PWCF material

\begin{tabular}{|c|c|c|c|c|c|}
\hline Specimen \# & $\begin{array}{l}\text { Average } \\
\text { Thickness } \\
\text { (in) }\end{array}$ & $\begin{array}{l}\text { Average Notch } \\
\text { to Notch Width } \\
\text { (in) }\end{array}$ & $\begin{array}{c}\text { Failure } \\
\text { Stress } \\
\left(\mathrm{lbf} / \mathrm{in}^{2}\right) \\
\end{array}$ & $\begin{array}{l}\text { Max } \\
\text { Load } \\
(\mathrm{lbs}) \\
\end{array}$ & $\begin{array}{c}\text { Average Strain } \\
\text { Rate } \\
(\mathrm{rad} / \mathrm{sec})\end{array}$ \\
\hline HSR-CRF-NW-VN-static-18 & 0.110 & 1.212 & 17720.30 & 2354.47 & 0.0012 \\
\hline HSR-CRF-NW-VN-static-20 & 0.110 & 1.210 & 12120.32 & 2337.66 & 0.0016 \\
\hline HSR-CRF-NW-VN-R1-02 & 0.101 & 1.211 & 21540.41 & 2627.93 & 1.03 \\
\hline HSR-CRF-NW-VN-R1-03 & 0.110 & 1.209 & 20365.79 & 2716.80 & 0.96 \\
\hline HSR-CRF-NW-VN-R1-05 & 0.111 & 1.209 & 19014.59 & 2551.76 & 0.88 \\
\hline HSR-CRF-NW-VN-R10-04 & 0.103 & 1.211 & 20825.31 & 2602.54 & 7.27 \\
\hline HSR-CRF-NW-VN-R10-07 & 0.112 & 1.208 & 21377.63 & 2894.53 & 7.73 \\
\hline HSR-CRF-NW-VN-R10-19 & 0.110 & 1.214 & 20715.41 & 2767.58 & 8.26 \\
\hline HSR-CRF-NW-VN-R100-08 & 0.111 & 1.208 & 30510.31 & 4100.59 & 162.08 \\
\hline HSR-CRF-NW-VN-R100-09 & 0.110 & 1.211 & 31926.90 & 4265.63 & 155.36 \\
\hline HSR-CRF-NW-VN-R100-18 & 0.110 & 1.212 & 30670.92 & 4075.20 & 172.67 \\
\hline HSR-CRF-NW-VN-R250-10 & 0.104 & 1.214 & 40942.89 & 5166.99 & 350.35 \\
\hline HSR-CRF-NW-VN-R250-11 & 0.105 & 1.210 & 41049.28 & 5217.77 & 412.92 \\
\hline HSR-CRF-NW-VN-R250-13 & 0.112 & 1.209 & 42399.18 & 5725.59 & 380.32 \\
\hline HSR-CRF-NW-VN-R500-12 & 0.102 & 1.209 & 42598.28 & 5268.55 & 501.36 \\
\hline HSR-CRF-NW-VN-R500-14 & 0.102 & 1.210 & 49507.61 & 6119.14 & 494.00 \\
\hline HSR-CRF-NW-VN-R500-15 & 0.111 & 1.209 & 45828.37 & 6131.84 & 479.94 \\
\hline
\end{tabular}


Stress-Strain and Strain Rate-Strain behavior of Newport NB321/7781 SWGF at different stroke rates

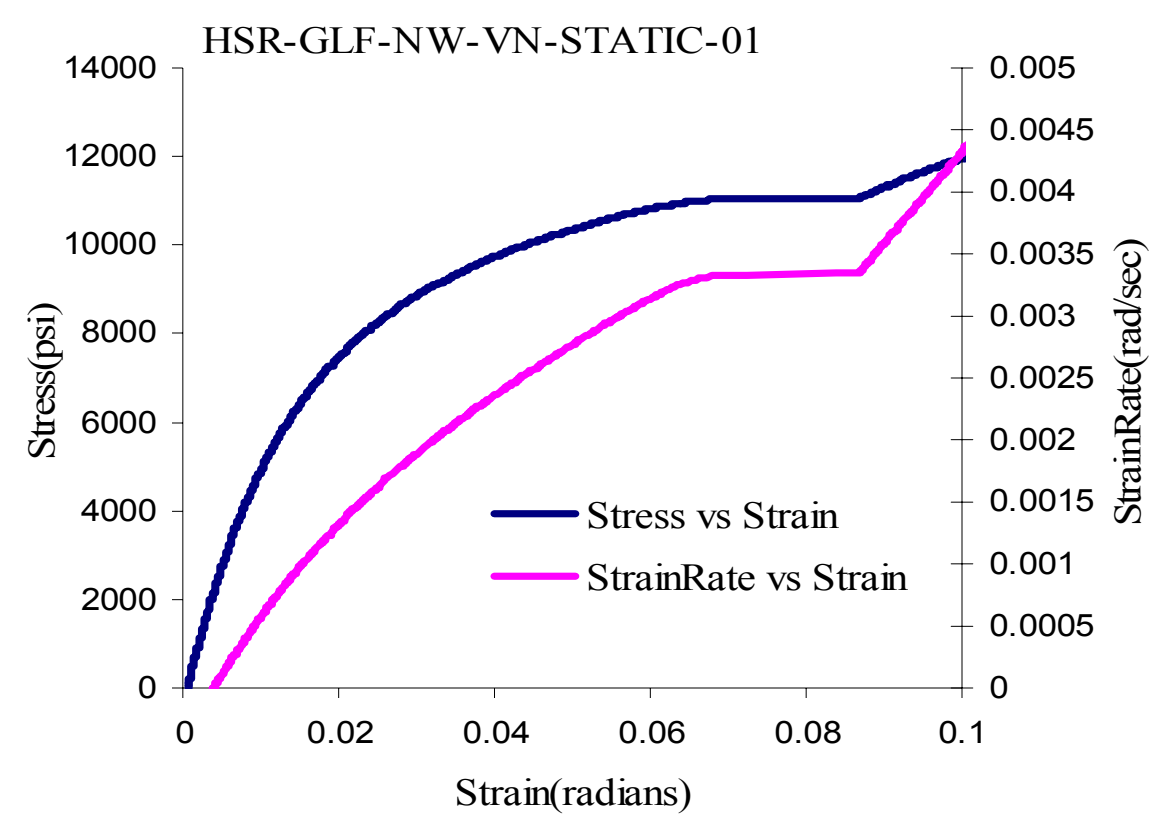

Figure 107. Stress-Strain and Strain Rate-Strain behavior of Newport SWGF at $0.00083 \mathrm{in} / \mathrm{s}$ stroke rate

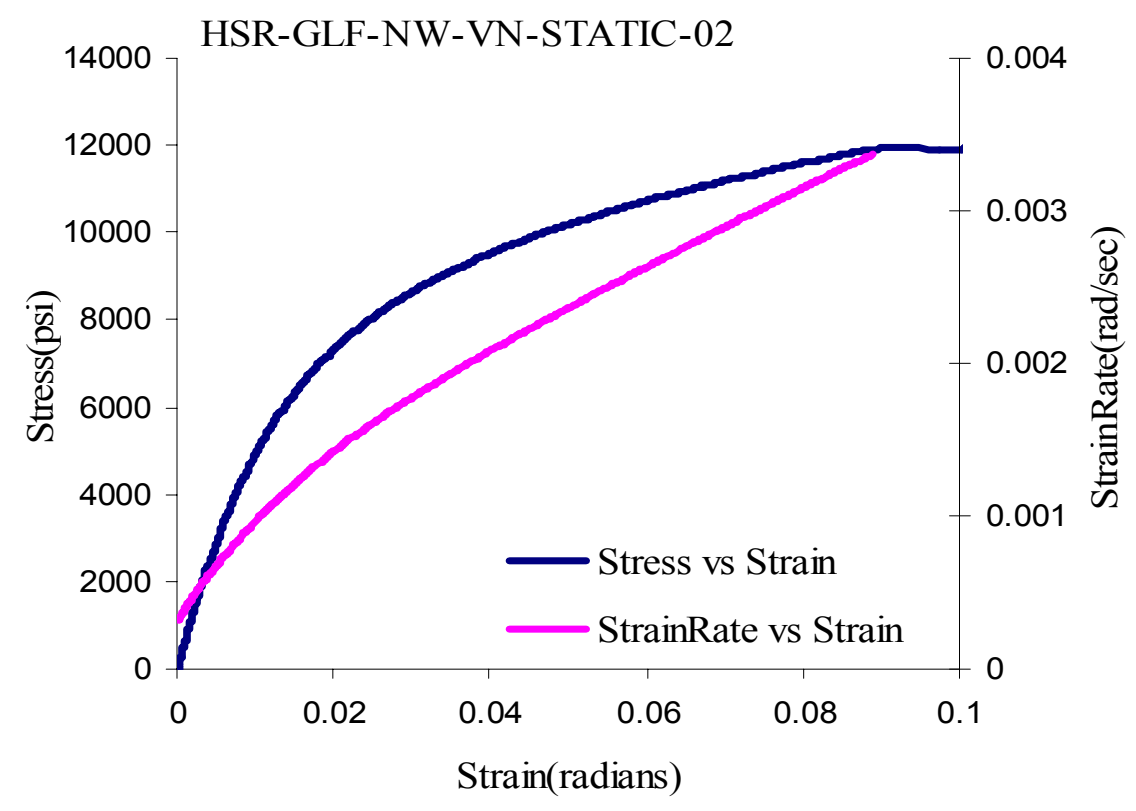

Figure 108. Stress-Strain and Strain Rate-Strain behavior of Newport SWGF at $0.00083 \mathrm{in} / \mathrm{s}$ stroke rate 


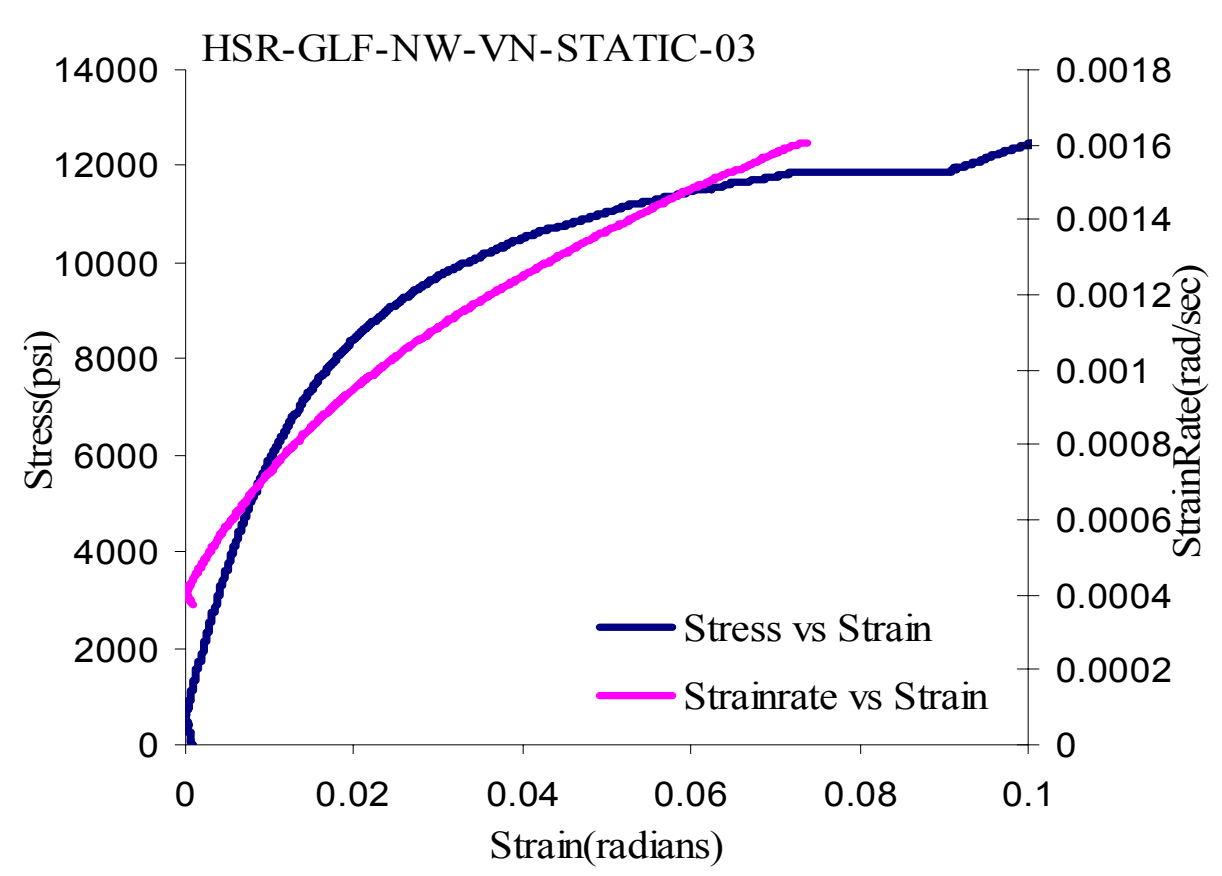

Figure 109. Stress-Strain and Strain Rate-Strain behavior of Newport SWGF at $0.00083 \mathrm{in} / \mathrm{s}$ stroke rate

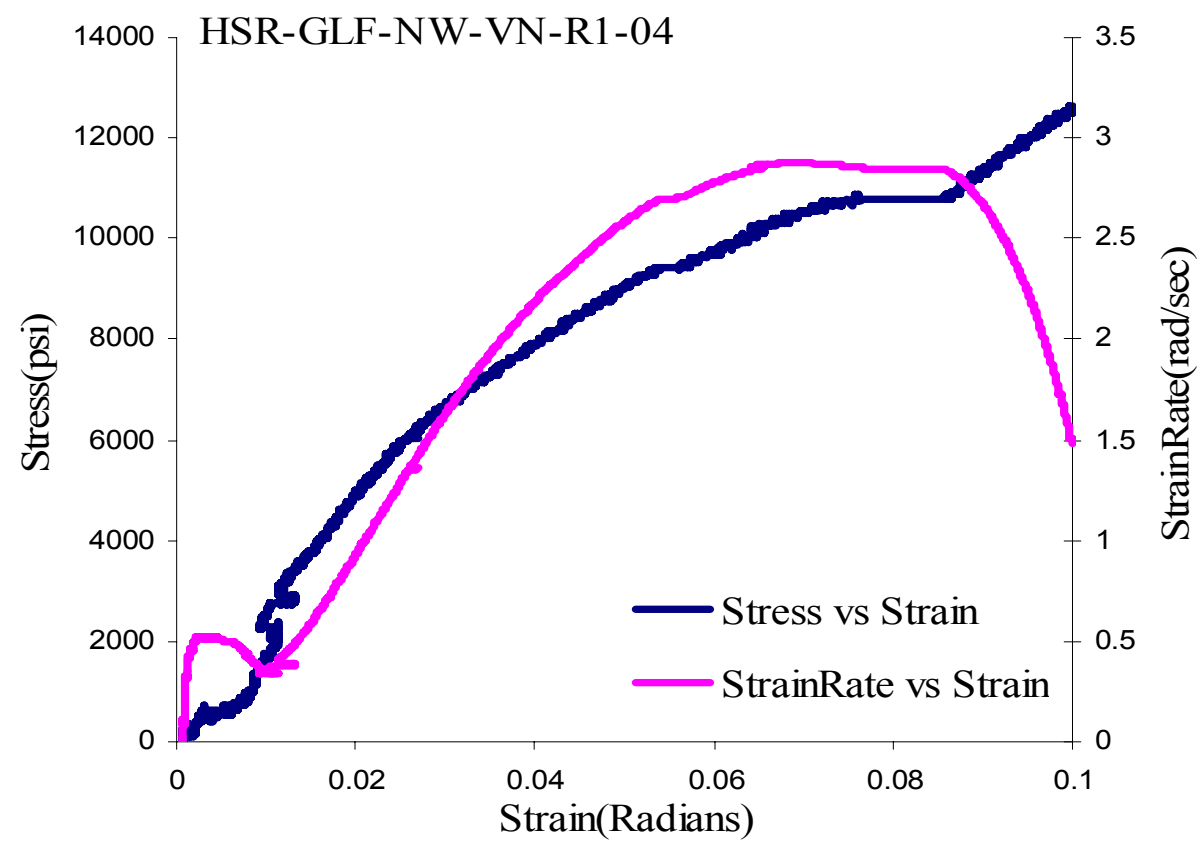

Figure 110. Stress-Strain and Strain Rate-Strain behavior of Newport SWGF at 1 in/s stroke rate 


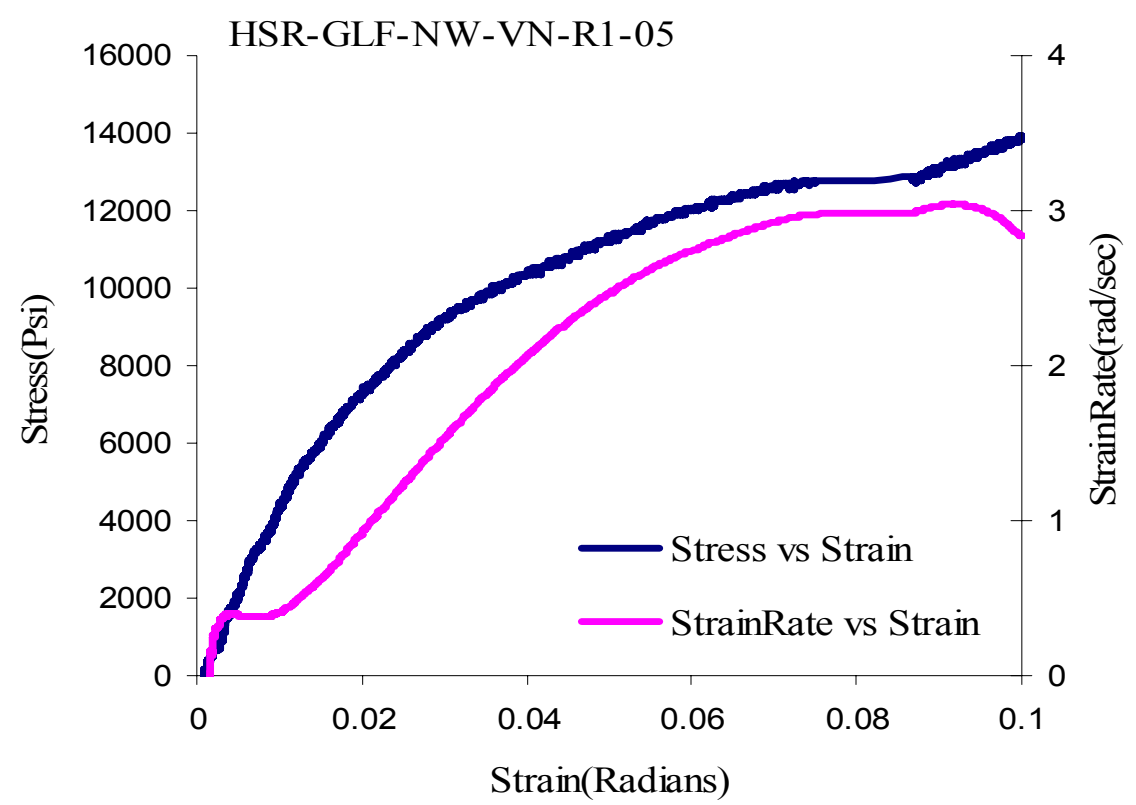

Figure 111. Stress-Strain and Strain Rate-Strain behavior of Newport SWGF at 1 in/s stroke rate

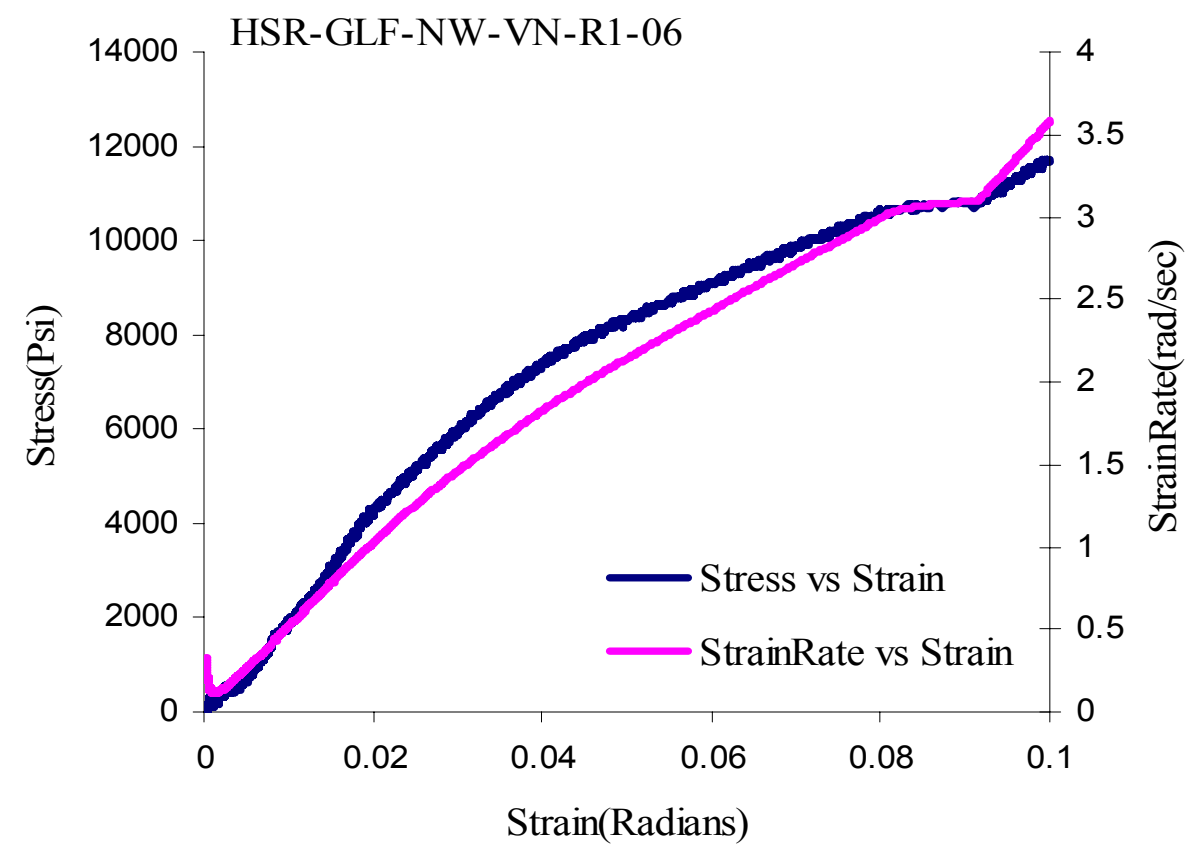

Figure 112. Stress-Strain and Strain Rate-Strain behavior of Newport SWGF at $1 \mathrm{in} / \mathrm{s}$ stroke rate 


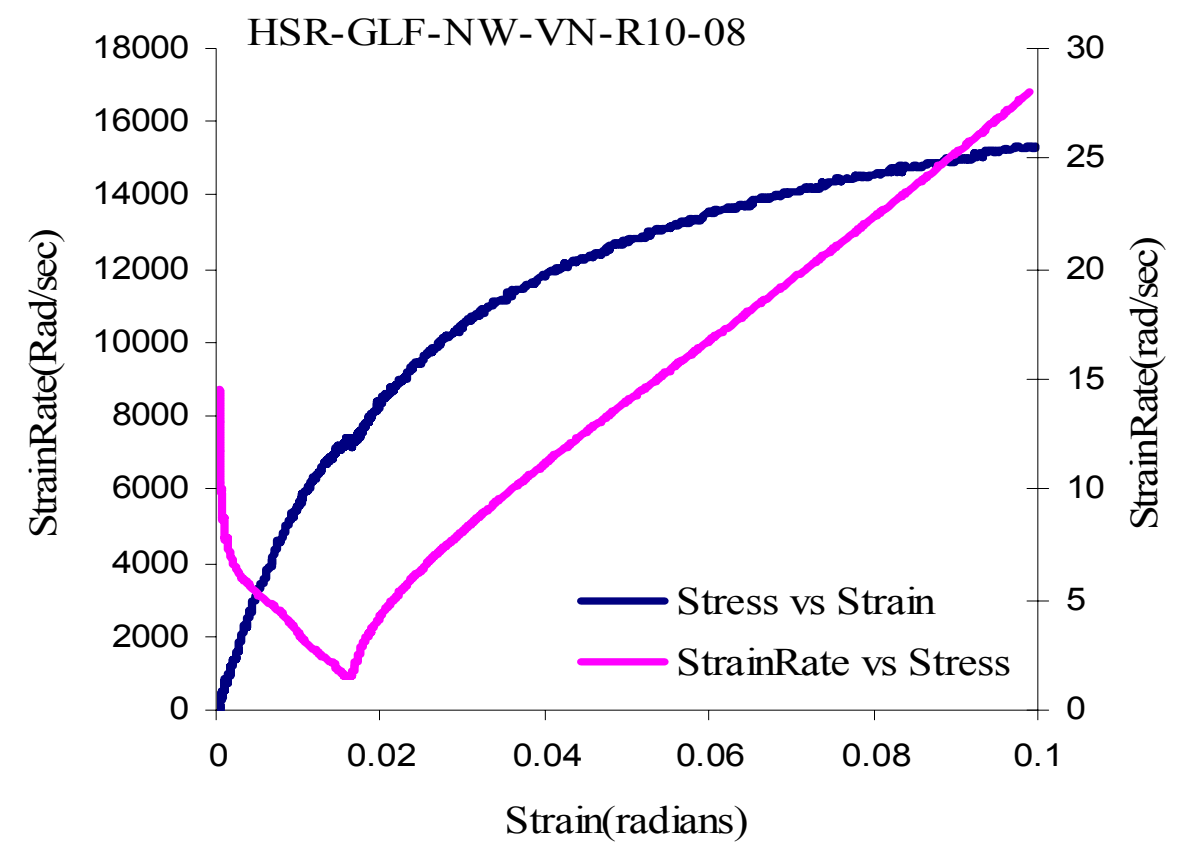

Figure 113. Stress-Strain and Strain Rate-Strain behavior of Newport SWGF at $10 \mathrm{in} / \mathrm{s}$ stroke rate

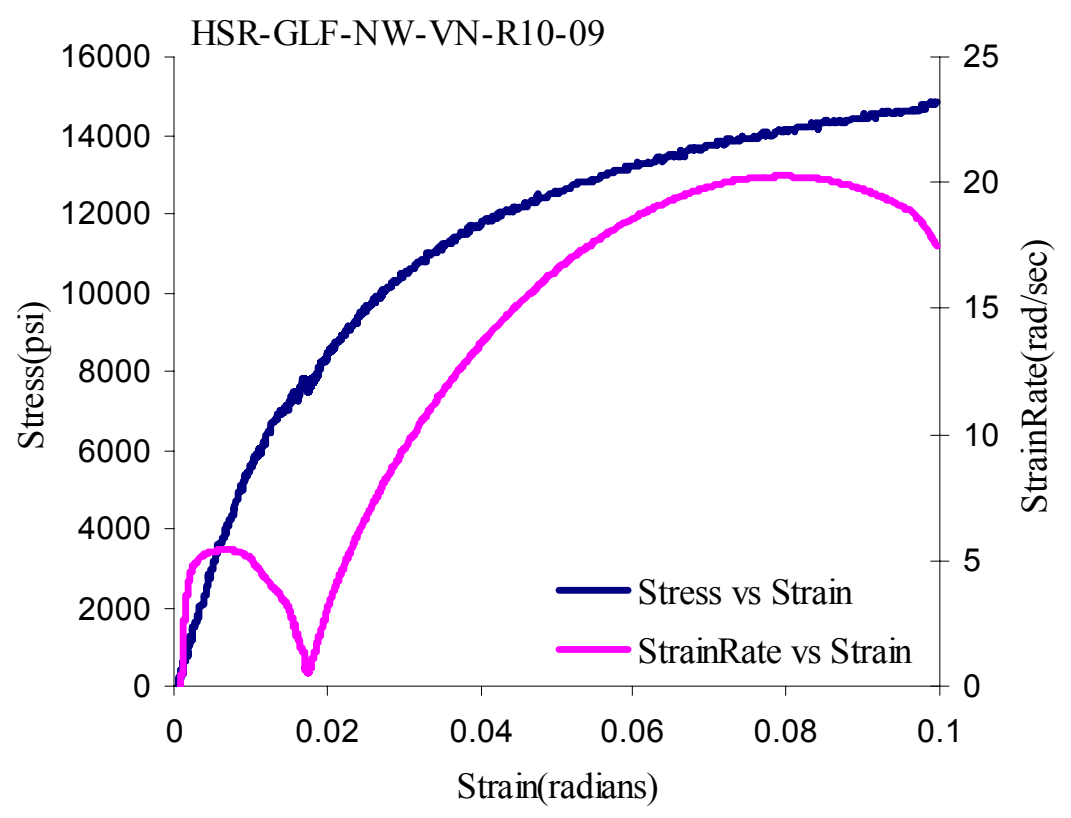

Figure 114. Stress-Strain and Strain Rate-Strain behavior of Newport SWGF at $10 \mathrm{in} / \mathrm{s}$ stroke rate 


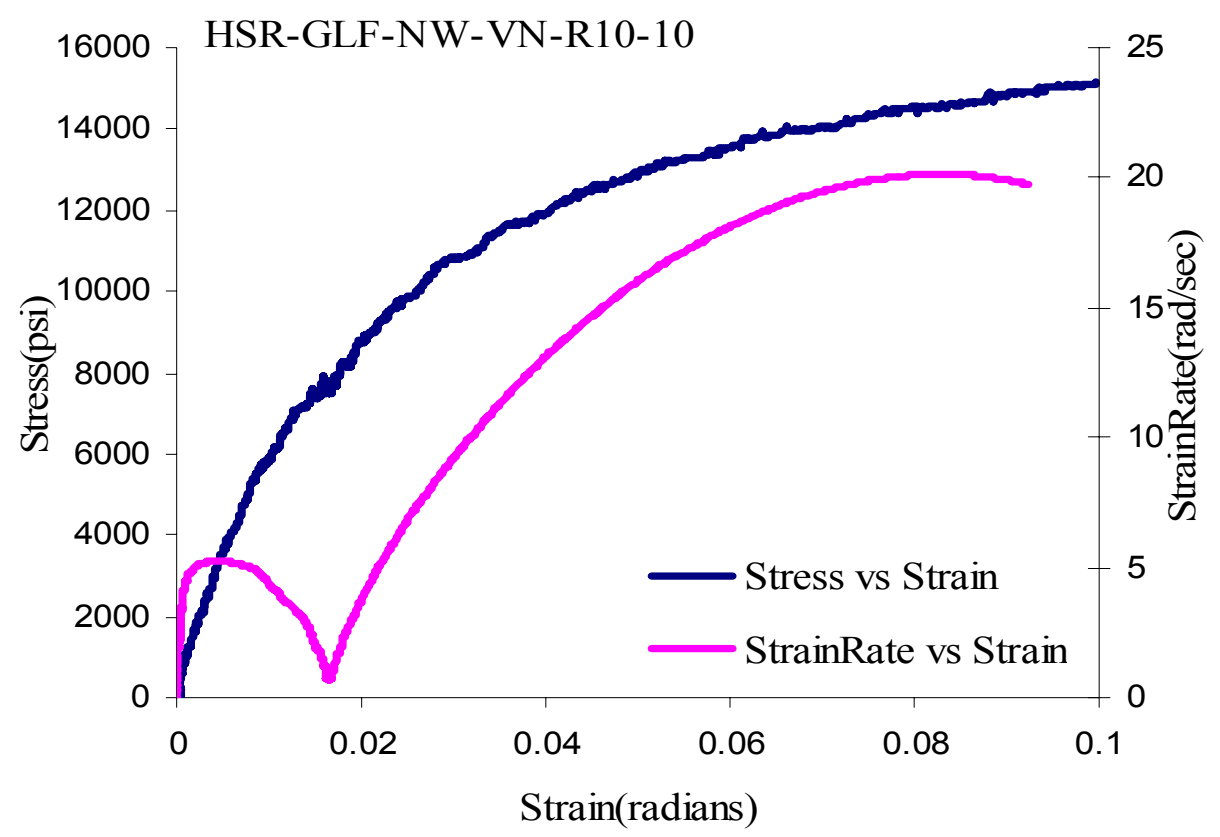

Figure 115. Stress-Strain and Strain Rate-Strain behavior of Newport SWGF at $10 \mathrm{in} / \mathrm{s}$ stroke rate

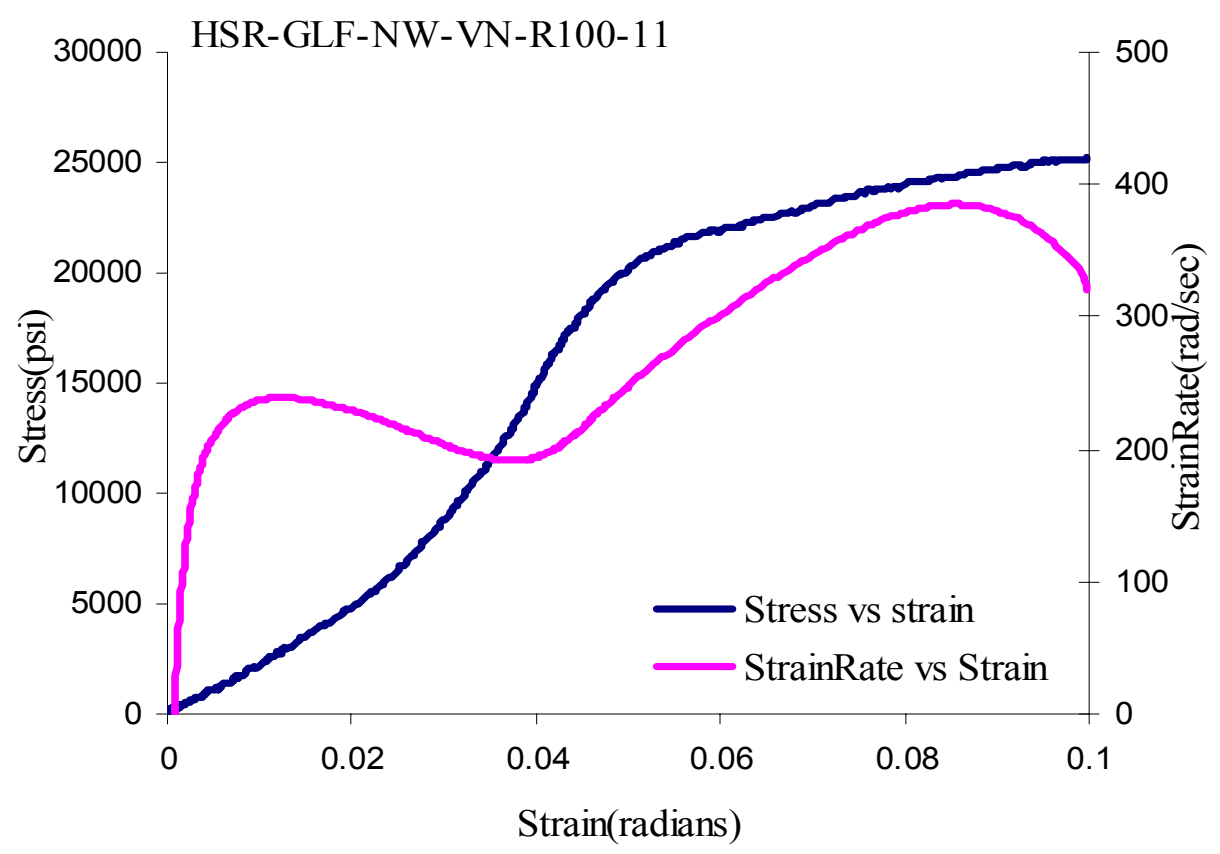

Figure 116. Stress-Strain and Strain Rate-Strain behavior of Newport SWGF at $100 \mathrm{in} / \mathrm{s}$ stroke rate 


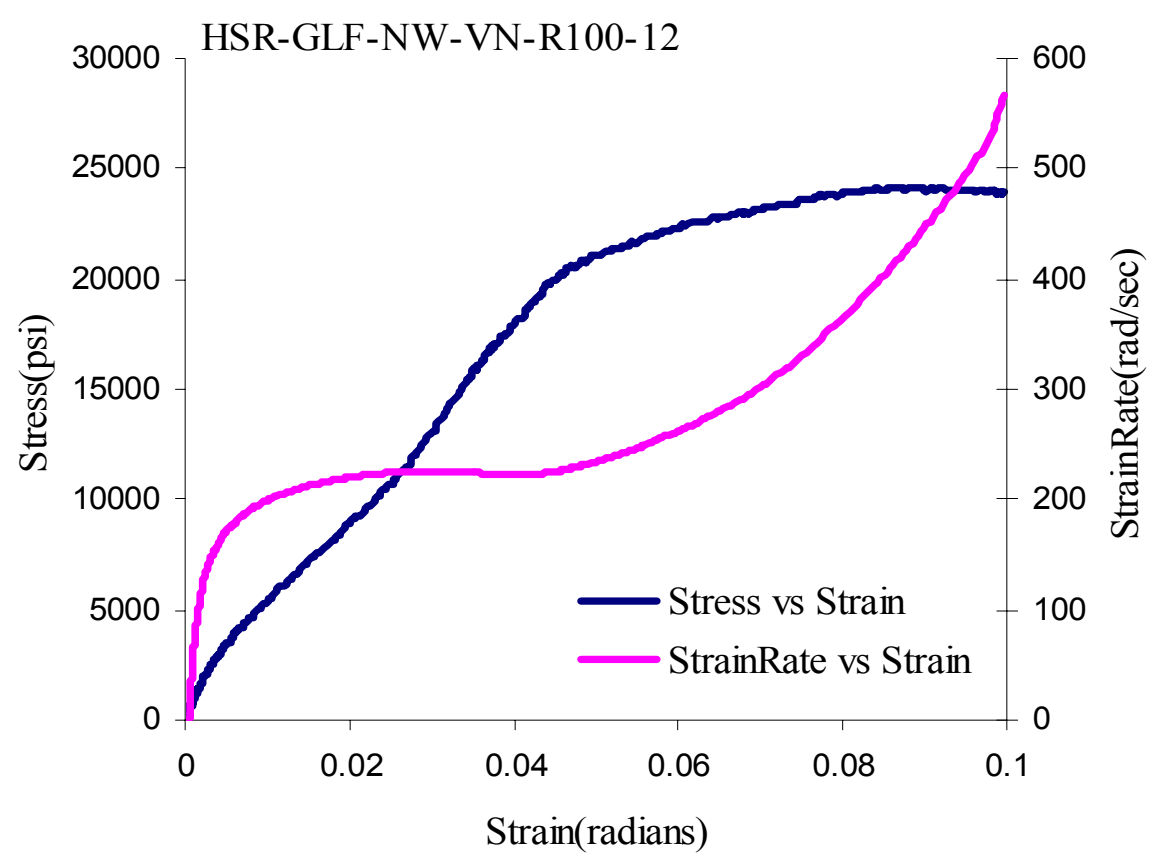

Figure 117. Stress-Strain and Strain Rate-Strain behavior of Newport SWGF at $100 \mathrm{in} / \mathrm{s}$ stroke rate

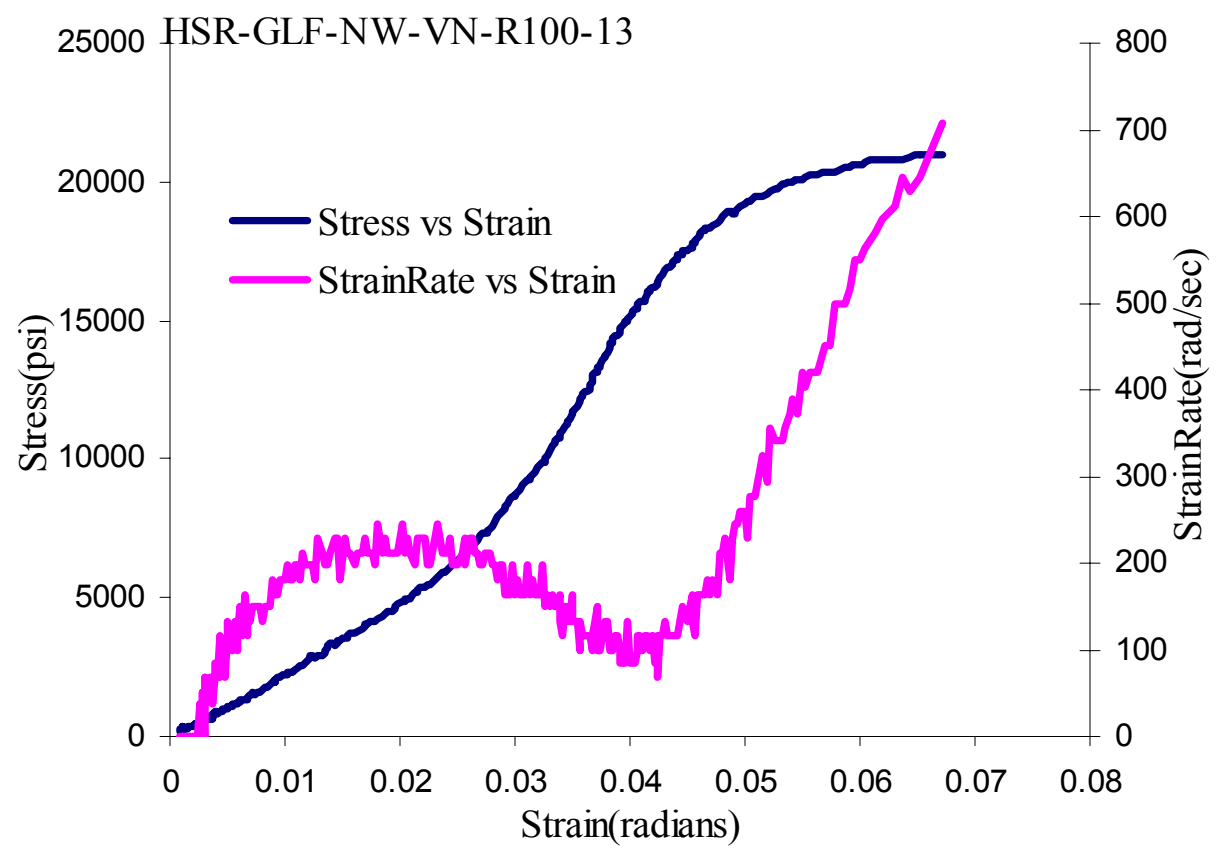

Figure 118. Stress-Strain and Strain Rate-Strain behavior of Newport SWGF at 100 in/s stroke rate 


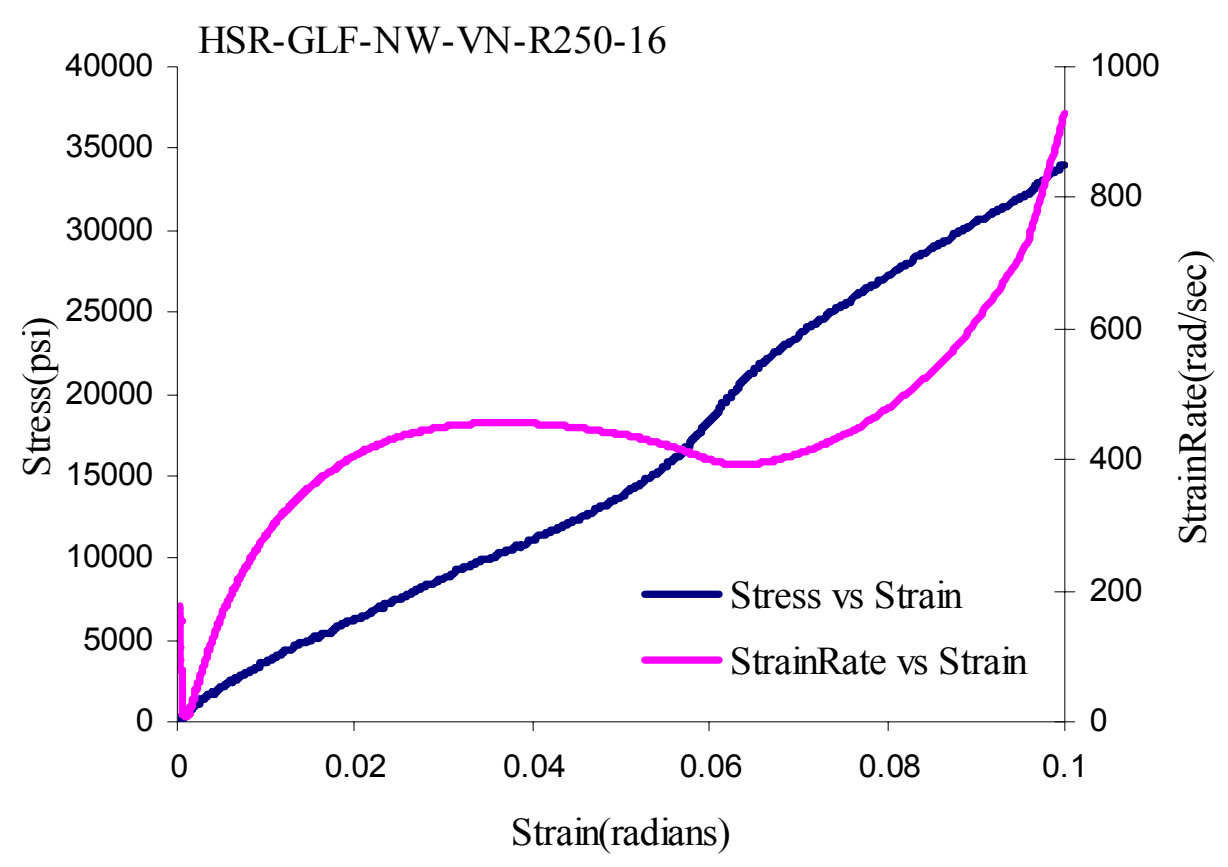

Figure 119. Stress-Strain and Strain Rate-Strain behavior of Newport SWGF at $250 \mathrm{in} / \mathrm{s}$ stroke rate

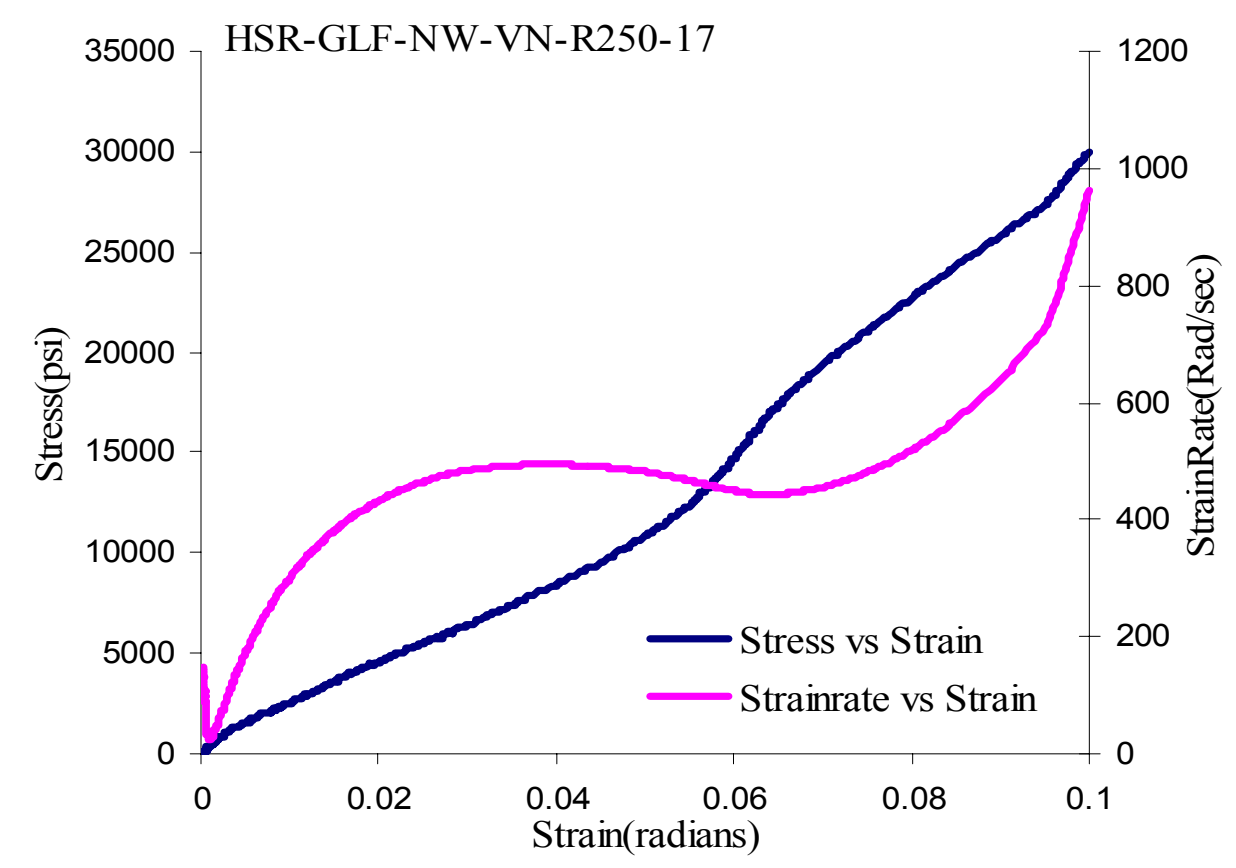

Figure 120. Stress-Strain and Strain Rate-Strain behavior of Newport SWGF at $250 \mathrm{in} / \mathrm{s}$ stroke rate 


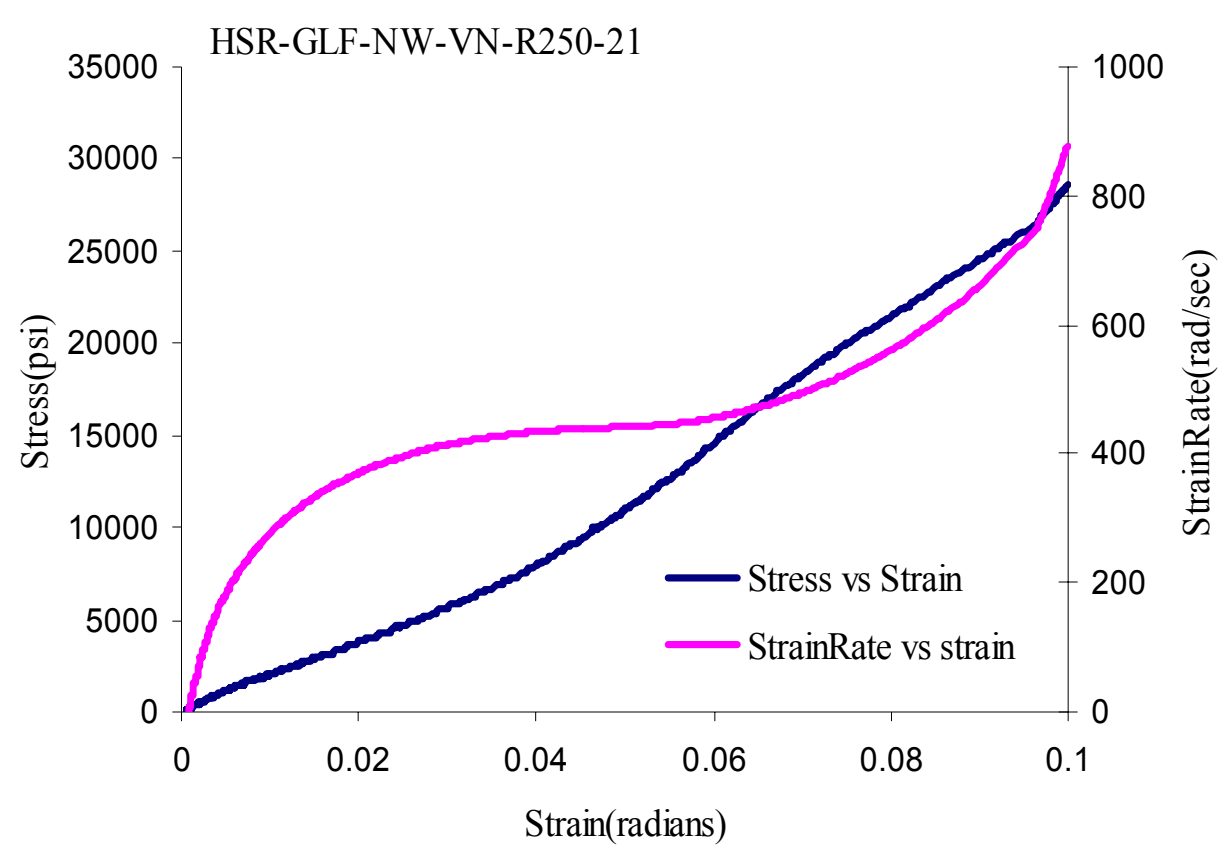

Figure 121. Stress-Strain and Strain Rate-Strain behavior of Newport SWGF at $250 \mathrm{in} / \mathrm{s}$ stroke rate

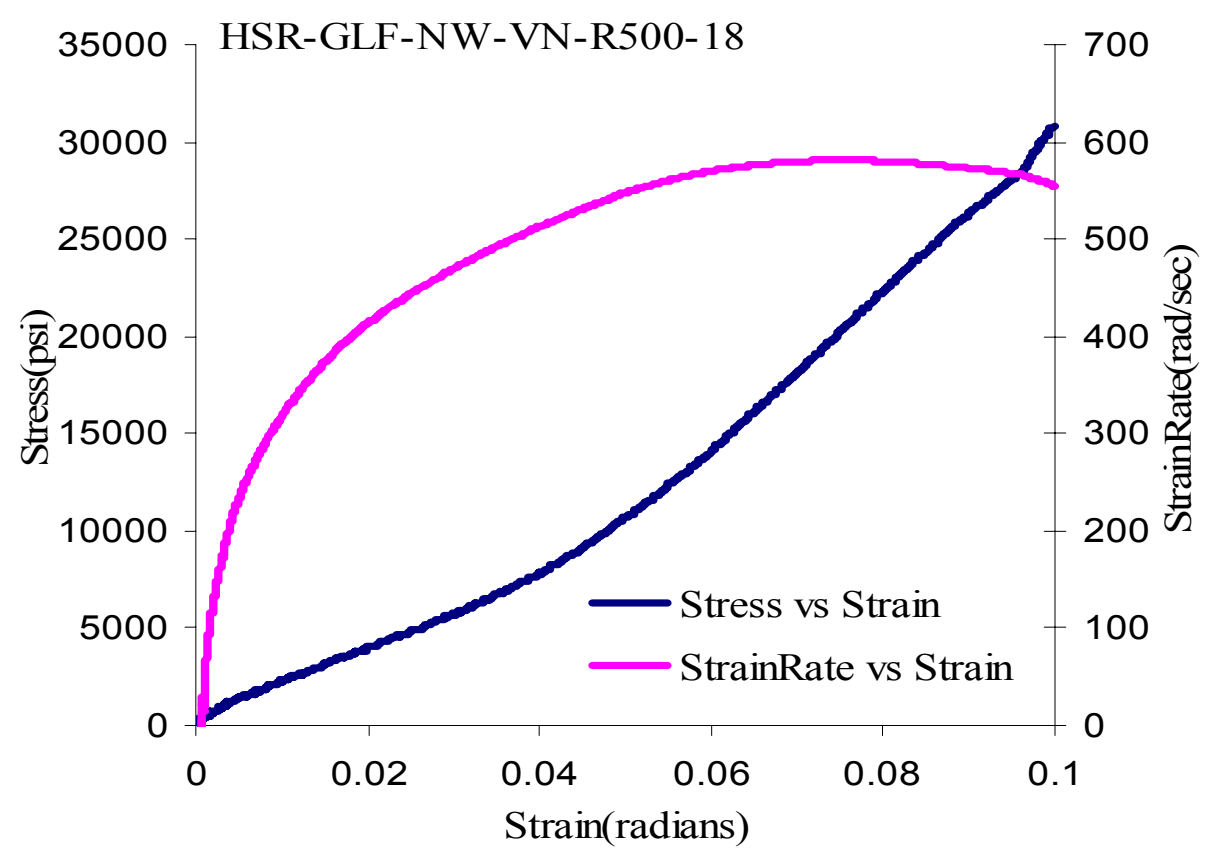

Figure 122. Stress-Strain and Strain Rate-Strain behavior of Newport SWGF at 500 in/s stroke rate 


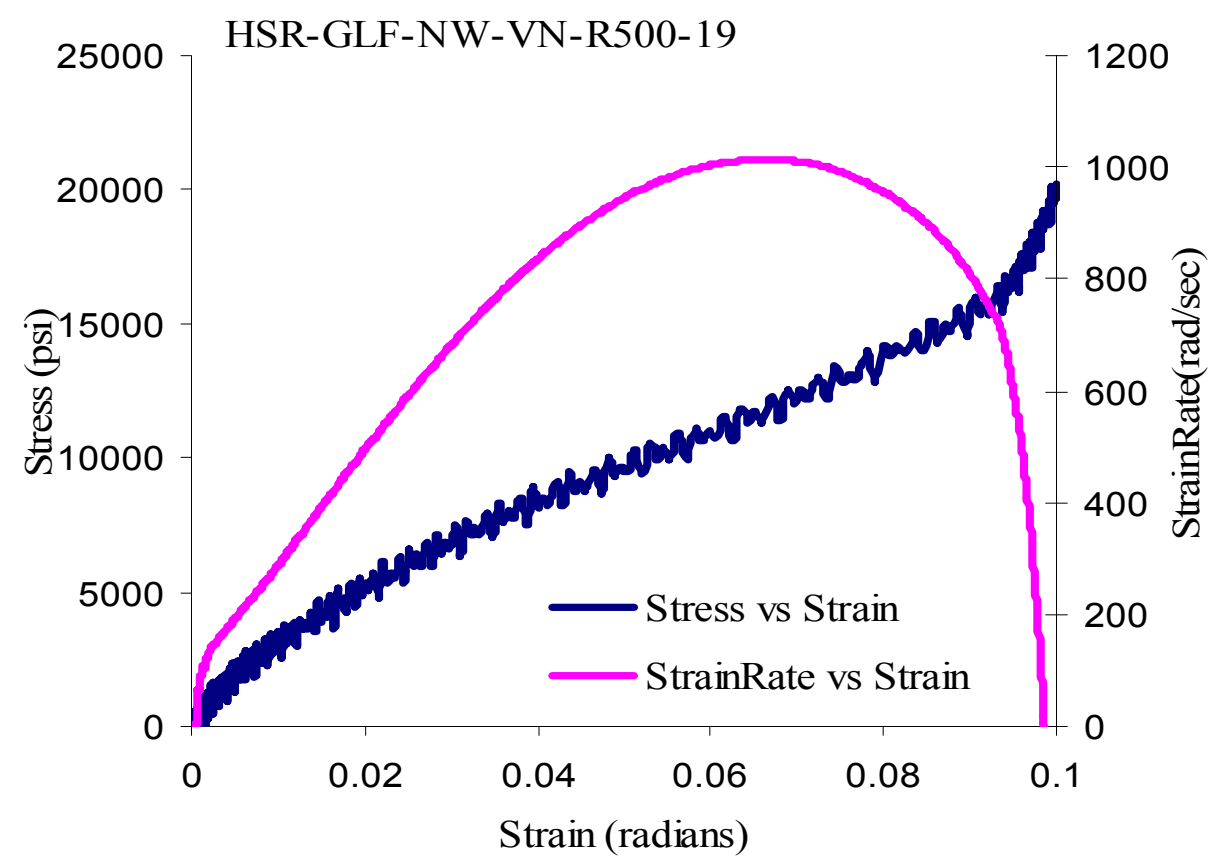

Figure 123. Stress-Strain and Strain Rate-Strain behavior of Newport SWGF at 500 in/s stroke rate

Failure Patterns of Newport NB321/7781 SWGF Specimens at different Stroke Rates

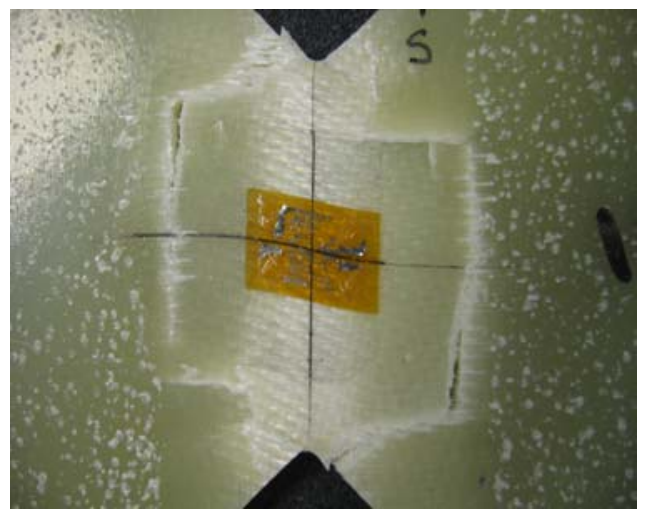

Figure 124. Failure mode of Newport SWGF material at $0.00083 \mathrm{in} / \mathrm{sec}$. 


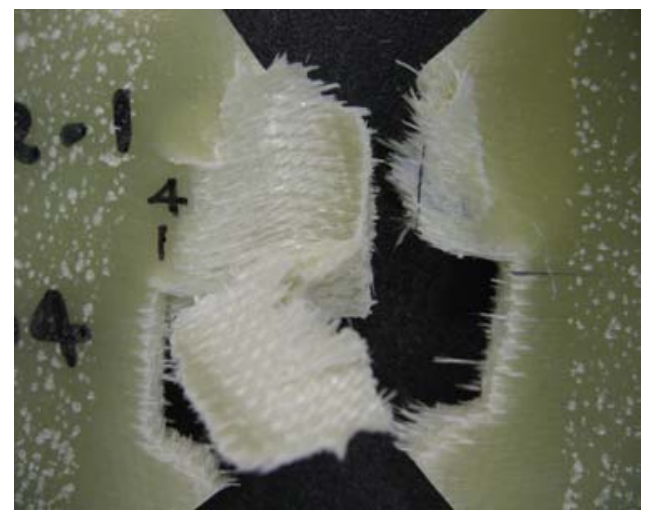

Figure 125. Failure mode of Newport SWGF material at $1 \mathrm{in} / \mathrm{sec}$.

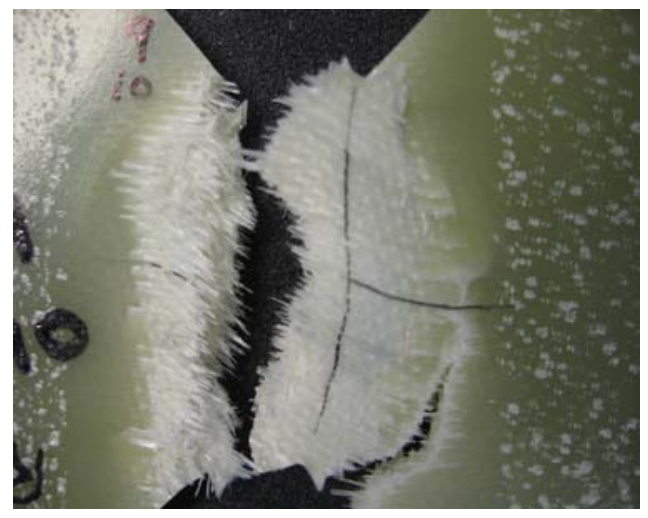

Figure 126. Failure mode of Newport SWGF material at $10 \mathrm{in} / \mathrm{sec}$.

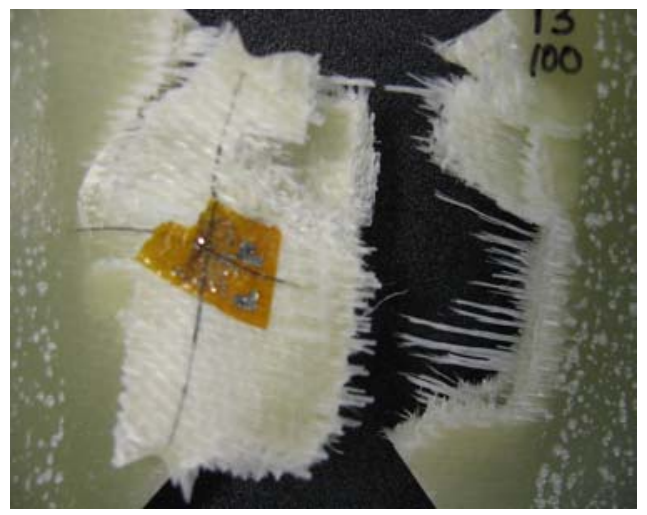

Figure 127. Failure mode of Newport SWGF material at $100 \mathrm{in} / \mathrm{sec}$. 


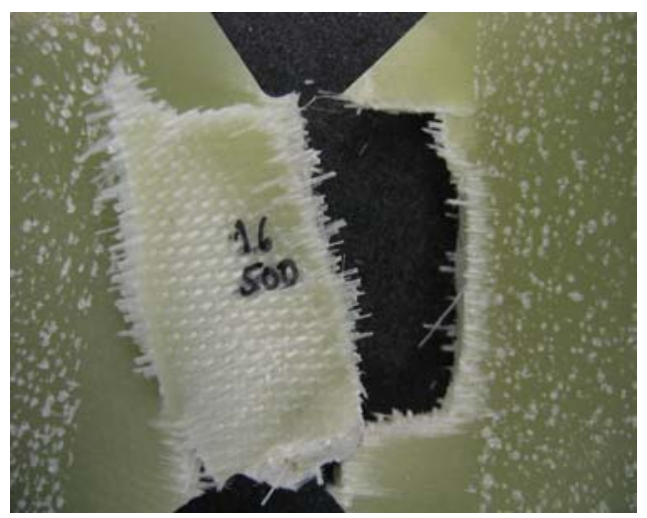

Figure 128. Failure mode of Newport SWGF material at $250 \mathrm{in} / \mathrm{sec}$.

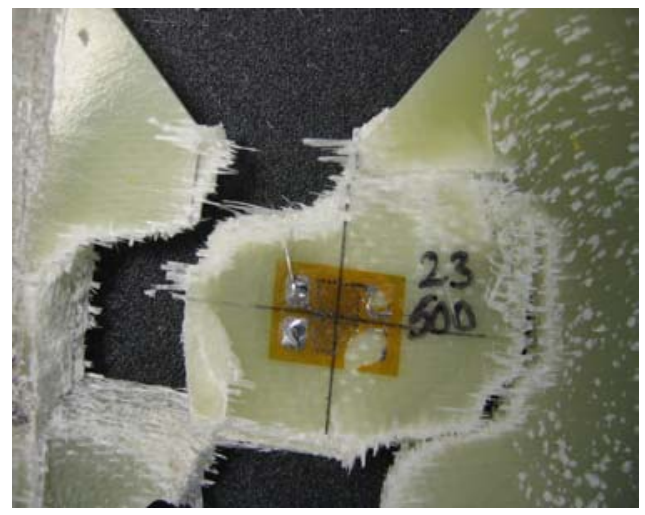

Figure 129. Failure mode of Newport SWGF material at $500 \mathrm{in} / \mathrm{sec}$. 
Table 11

Summary of test data for Newport SWGF material

\begin{tabular}{|c|c|c|c|c|c|}
\hline Specimen \# & $\begin{array}{l}\text { Average } \\
\text { Thickness } \\
\text { (in) }\end{array}$ & $\begin{array}{l}\text { Average Notch } \\
\text { to Notch Width } \\
\text { (in) }\end{array}$ & $\begin{array}{l}\text { Failure } \\
\text { Stress } \\
\left(\mathrm{lbf} / \mathrm{in}^{2}\right)\end{array}$ & $\begin{array}{l}\text { Max } \\
\text { Load } \\
\text { (lbs) }\end{array}$ & $\begin{array}{c}\text { Average } \\
\text { Strain Rate } \\
(\mathrm{rad} / \mathrm{sec})\end{array}$ \\
\hline HSR-GLF-NW-VN-static-01 & 0.130975 & 1.209 & 18057.66 & 2861.18 & 0.00051 \\
\hline HSR-GLF-NW-VN-static-02 & 0.131275 & 1.209 & 17236.73 & 2737.34 & 0.00184 \\
\hline HSR-GLF-NW-VN-static-03 & 0.12125 & 1.208 & 18465.40 & 2702.94 & 0.00099 \\
\hline HSR-GLF-NW-VN-R1-04 & 0.1309 & 1.221 & 19857.77 & 3173.82 & 1.13 \\
\hline HSR-GLF-NW-VN-R1-05 & 0.12825 & 1.222 & 19595.41 & 3072.26 & 1.16 \\
\hline HSR-GLF-NW-VN-R1-06 & 0.133175 & 1.223 & 20159.60 & 3275.39 & 1.05 \\
\hline HSR-GLF-NW-VN-R10-08 & 0.13265 & 1.221 & 22329.94 & 3618.16 & 9.85 \\
\hline HSR-GLF-NW-VN-R10-09 & 0.133 & 1.221 & 21485.30 & 3491.21 & 6.88 \\
\hline HSR-GLF-NW-VN-R10-10 & 0.134325 & 1.221 & 22137.90 & 3630.85 & 7.06 \\
\hline HSR-GLF-NW-VN-R100-11 & 0.127825 & 1.222 & 29284.02 & 4583.00 & 220.94 \\
\hline HSR-GLF-NW-VN-R100-12 & 0.1346 & 1.221 & 28658.64 & 4709.96 & 225.76 \\
\hline HSR-GLF-NW-VN-R100-13 & 0.13435 & 1.221 & 28545.65 & 4684.57 & 155.90 \\
\hline HSR-GLF-NW-VN-R250-16 & 0.1295 & 1.221 & 42848.20 & 6779.29 & 335.30 \\
\hline HSR-GLF-NW-VN-R250-17 & 0.12975 & 1.221 & 42703.03 & 6766.60 & 371.59 \\
\hline HSR-GLF-NW-VN-R250-21 & 0.12775 & 1.216 & 42143.52 & 6550.78 & 351.39 \\
\hline HSR-GLF-NW-VN-R500-18 & 0.1315 & 1.221 & 46621.61 & 7490.23 & 374.71 \\
\hline HSR-GLF-NW-VN-R500-19 & 0.12525 & 1.221 & 45091.68 & 6855.46 & 447.97 \\
\hline HSR-GLF-NW-VN-R500-22 & 0.13075 & 1.220 & 35640.35 & 5687.51 & 465.62 \\
\hline
\end{tabular}




\section{Stress-Strain and Strain Rate-Strain behavior of Newport NCT/321/G150 Unitape at different stroke rates}

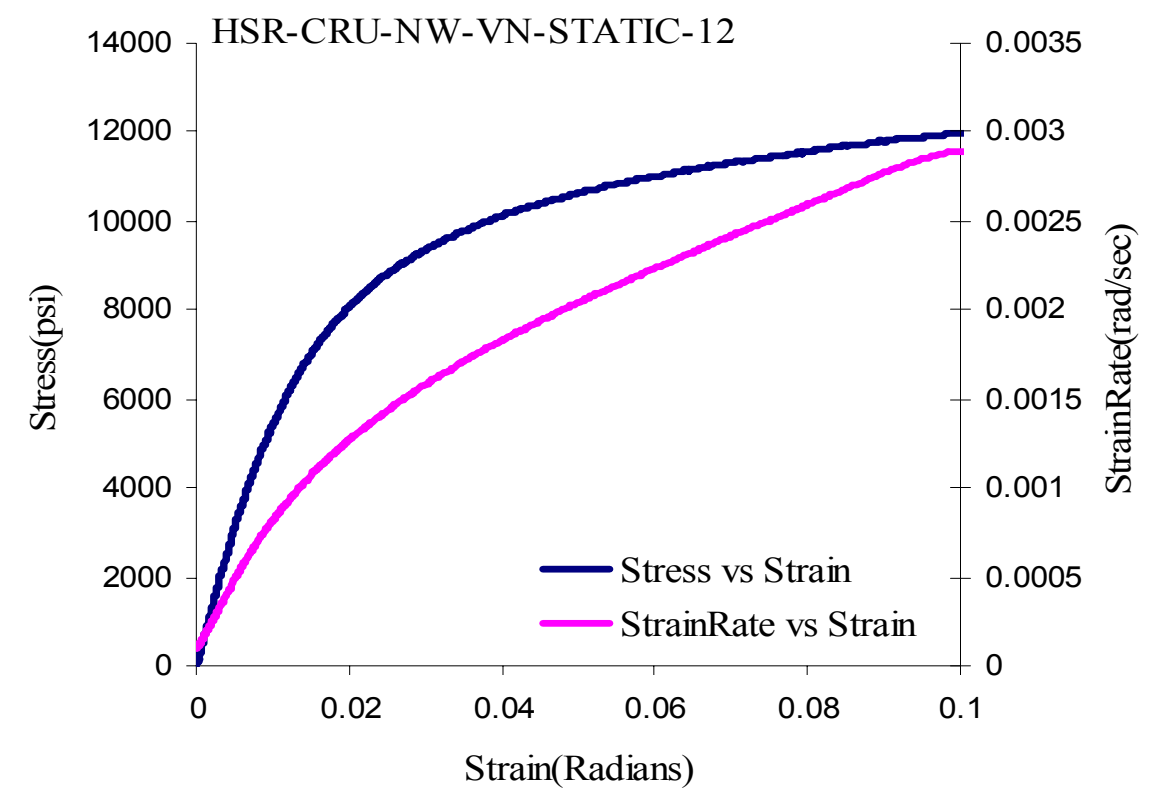

Figure 130. Stress-Strain and Strain Rate-Strain behavior of Newport Unitape at $0.00083 \mathrm{in} / \mathrm{s}$ stroke rate

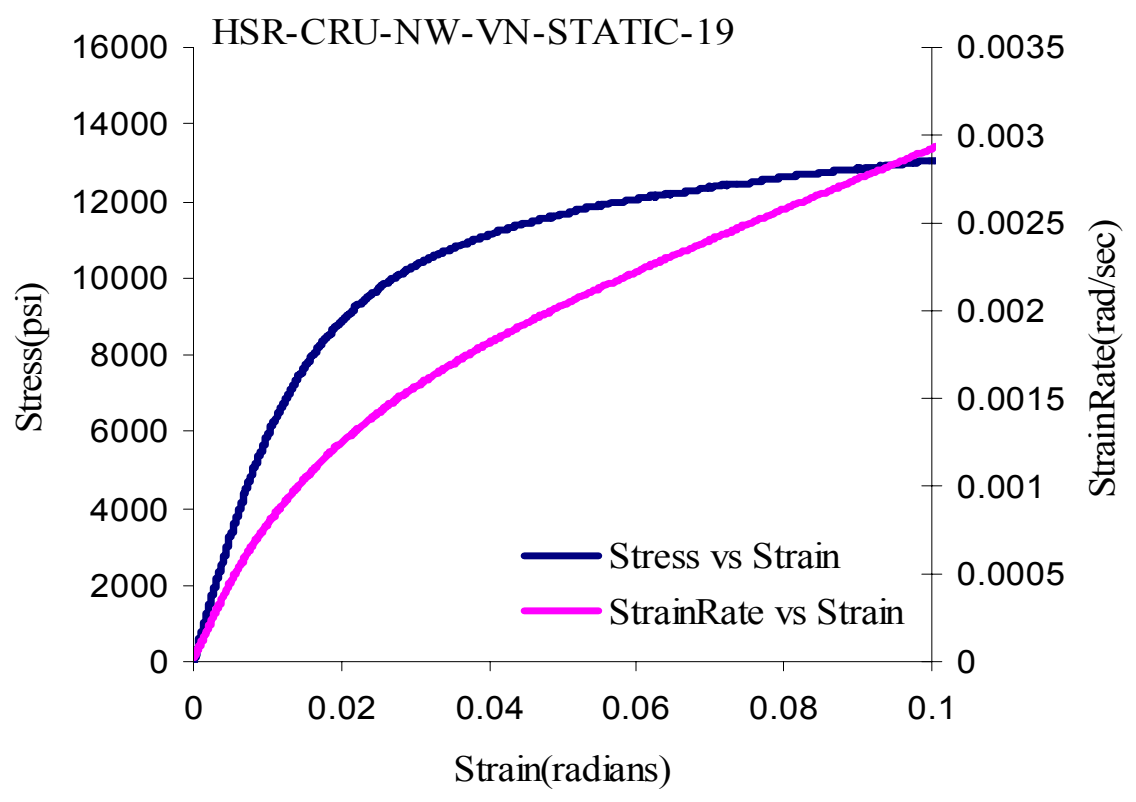

Figure 131. Stress-Strain and Strain Rate-Strain behavior of Newport Unitape at $0.00083 \mathrm{in} / \mathrm{s}$ stroke rate 


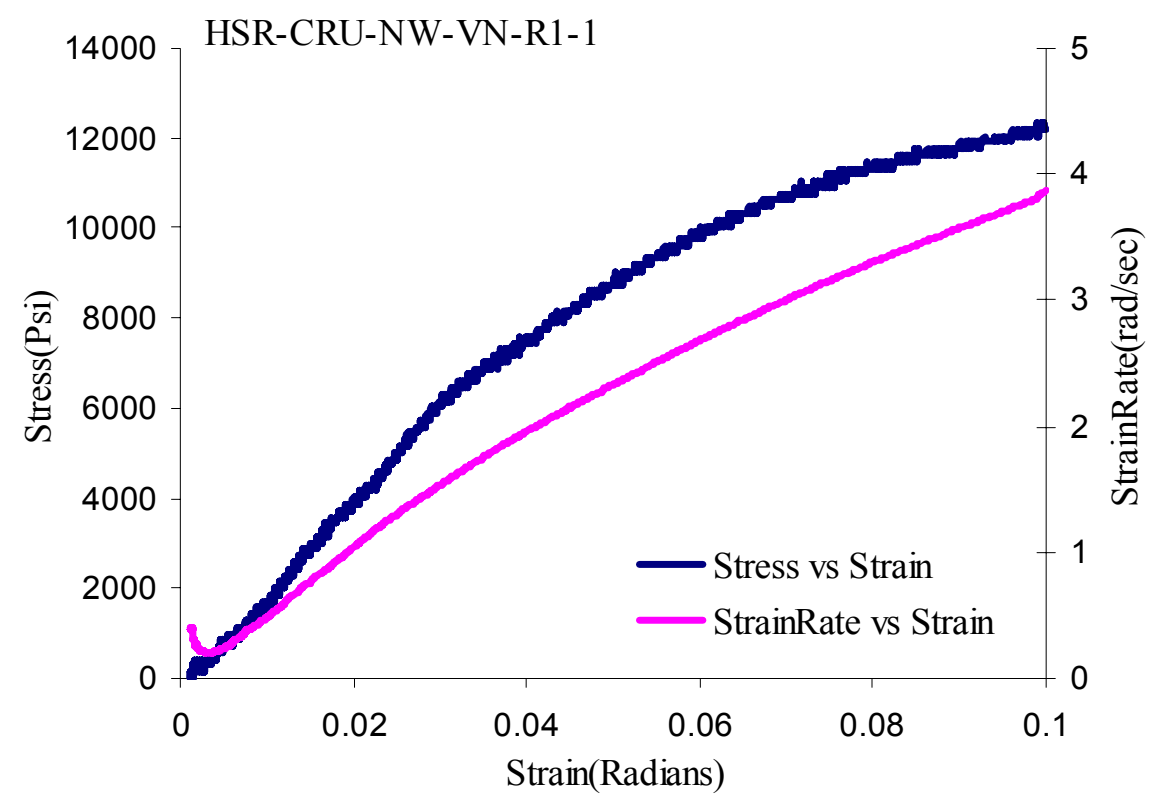

Figure 132. Stress-Strain and Strain Rate-Strain behavior of Newport Unitape at $1 \mathrm{in} / \mathrm{s}$ stroke rate

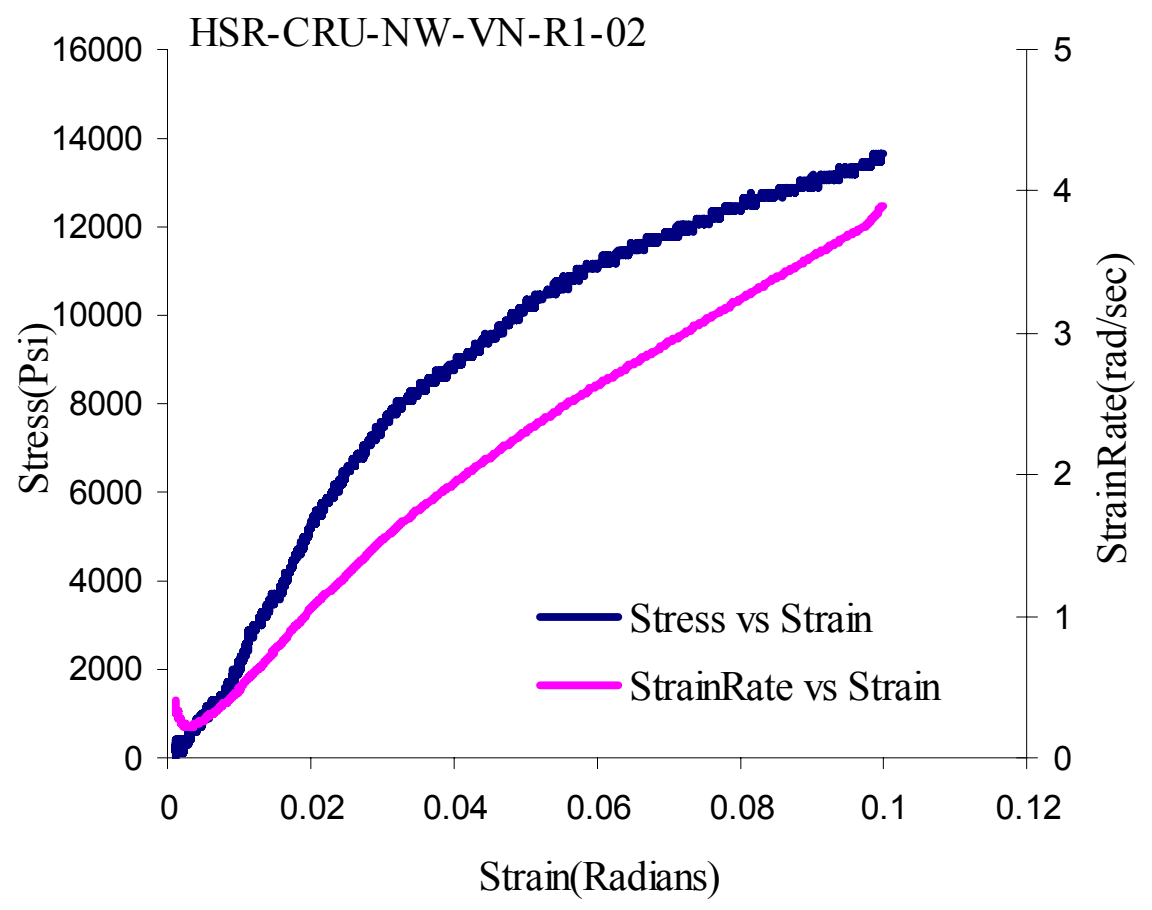

Figure 133. Stress-Strain and Strain Rate-Strain behavior of Newport Unitape at $1 \mathrm{in} / \mathrm{s}$ stroke rate 


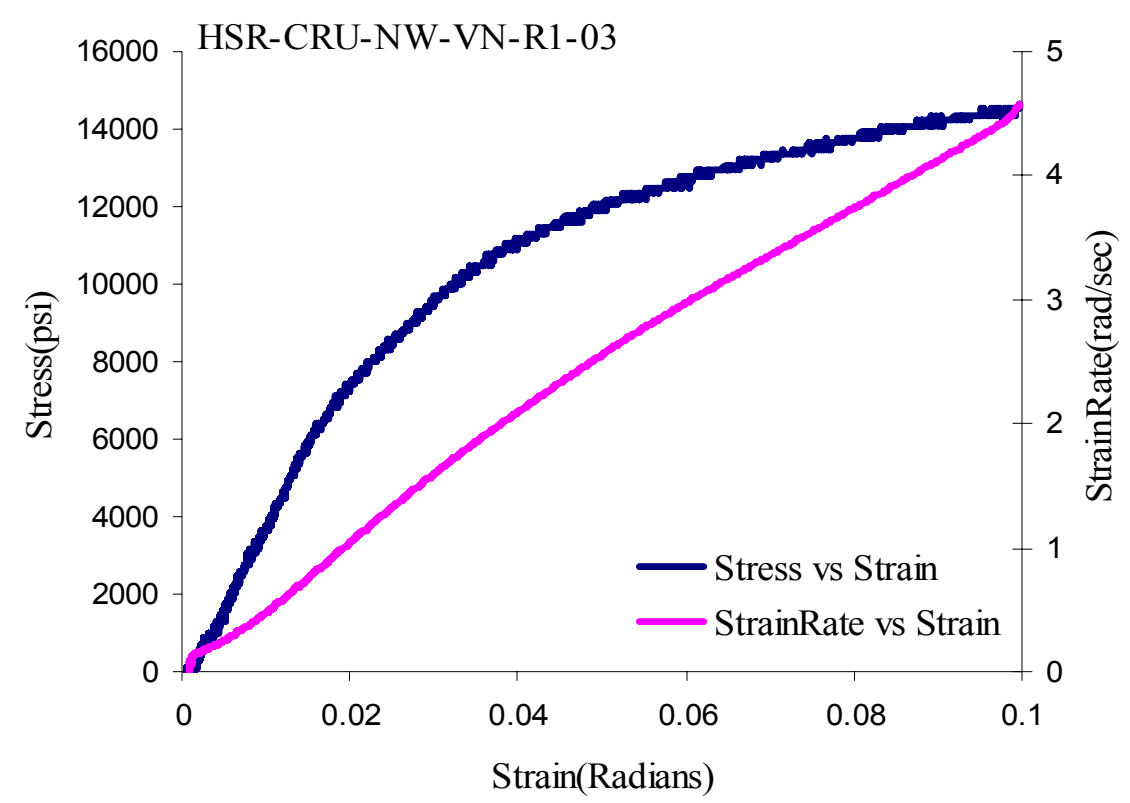

Figure 134. Stress-Strain and Strain Rate-Strain behavior of Newport Unitape at $1 \mathrm{in} / \mathrm{s}$ stroke rate

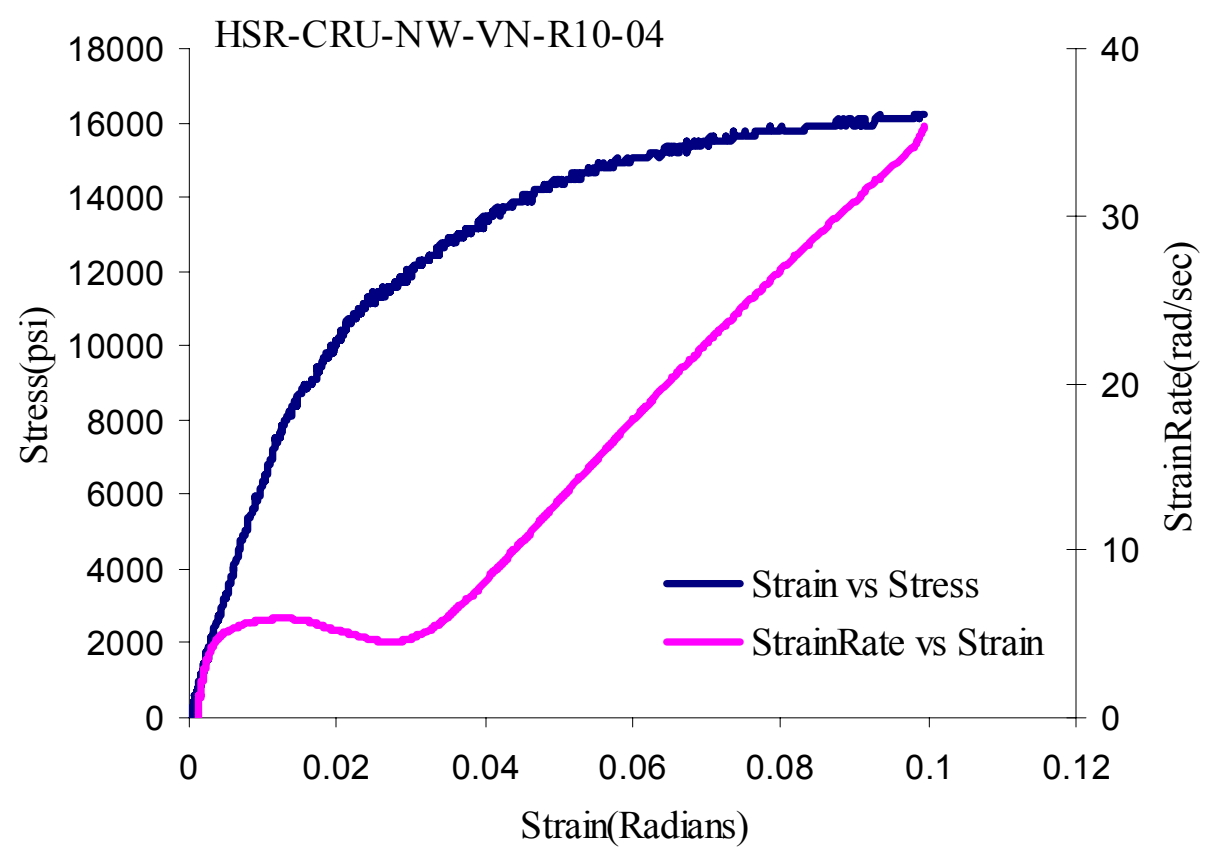

Figure 135. Stress-Strain and Strain Rate-Strain behavior of Newport Unitape at $10 \mathrm{in} / \mathrm{s}$ stroke rate 


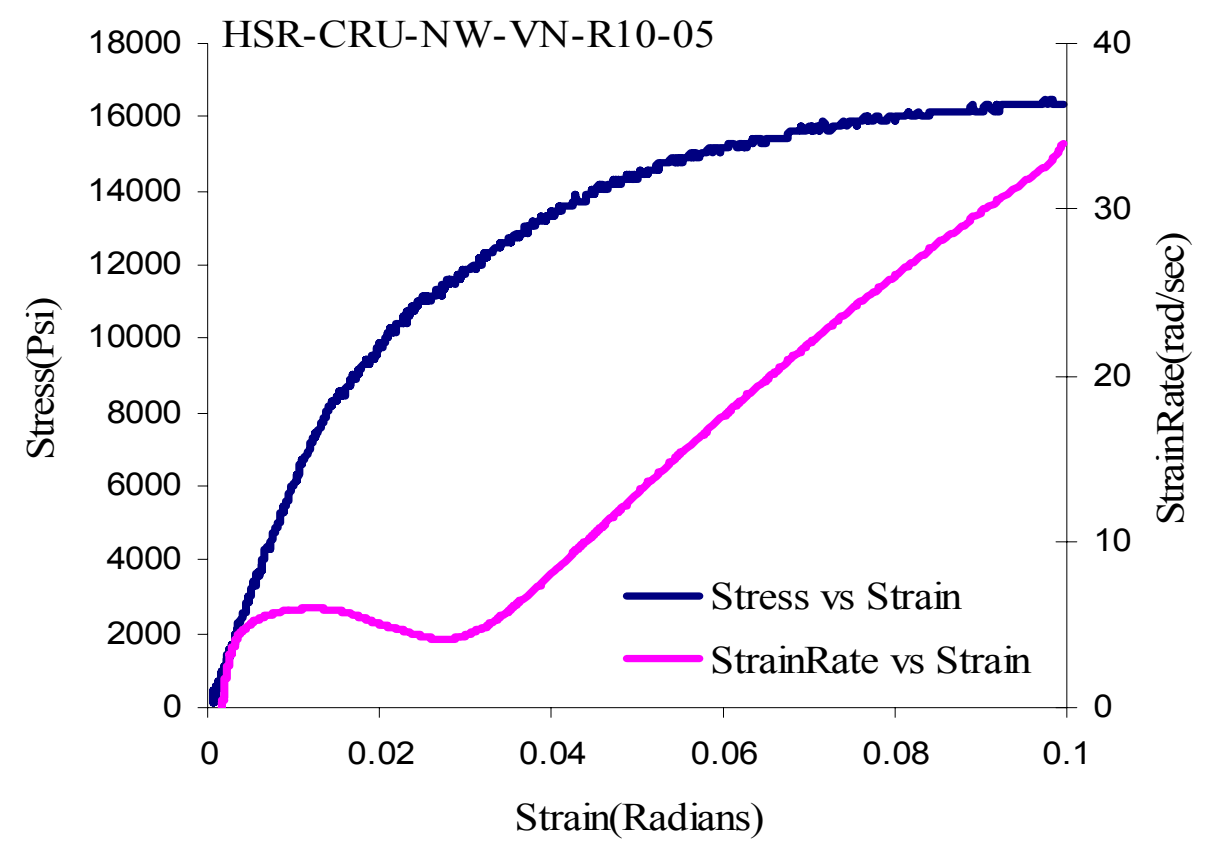

Figure 136. Stress-Strain and Strain Rate-Strain behavior of Newport Unitape at $10 \mathrm{in} / \mathrm{s}$ stroke rate

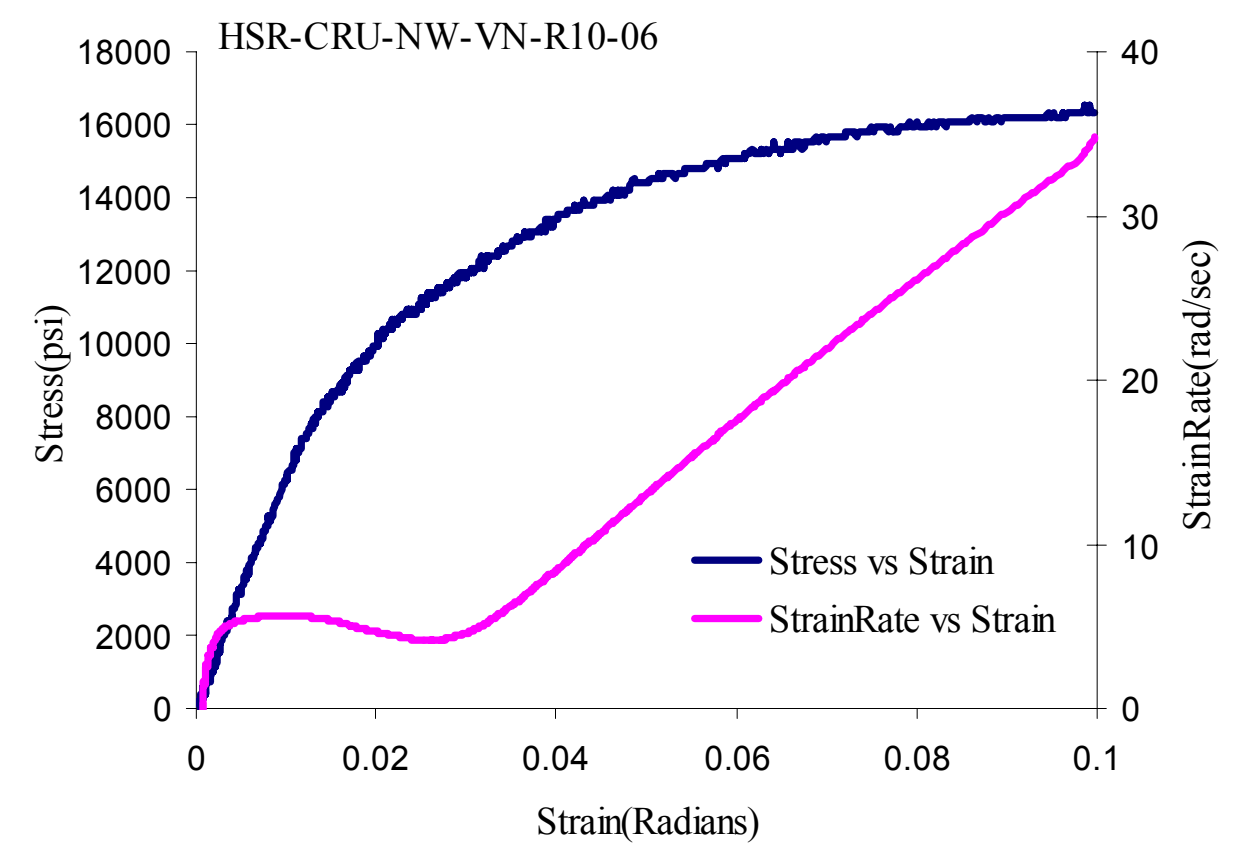

Figure 137. Stress-Strain and Strain Rate-Strain behavior of Newport Unitape at $10 \mathrm{in} / \mathrm{s}$ stroke rate 


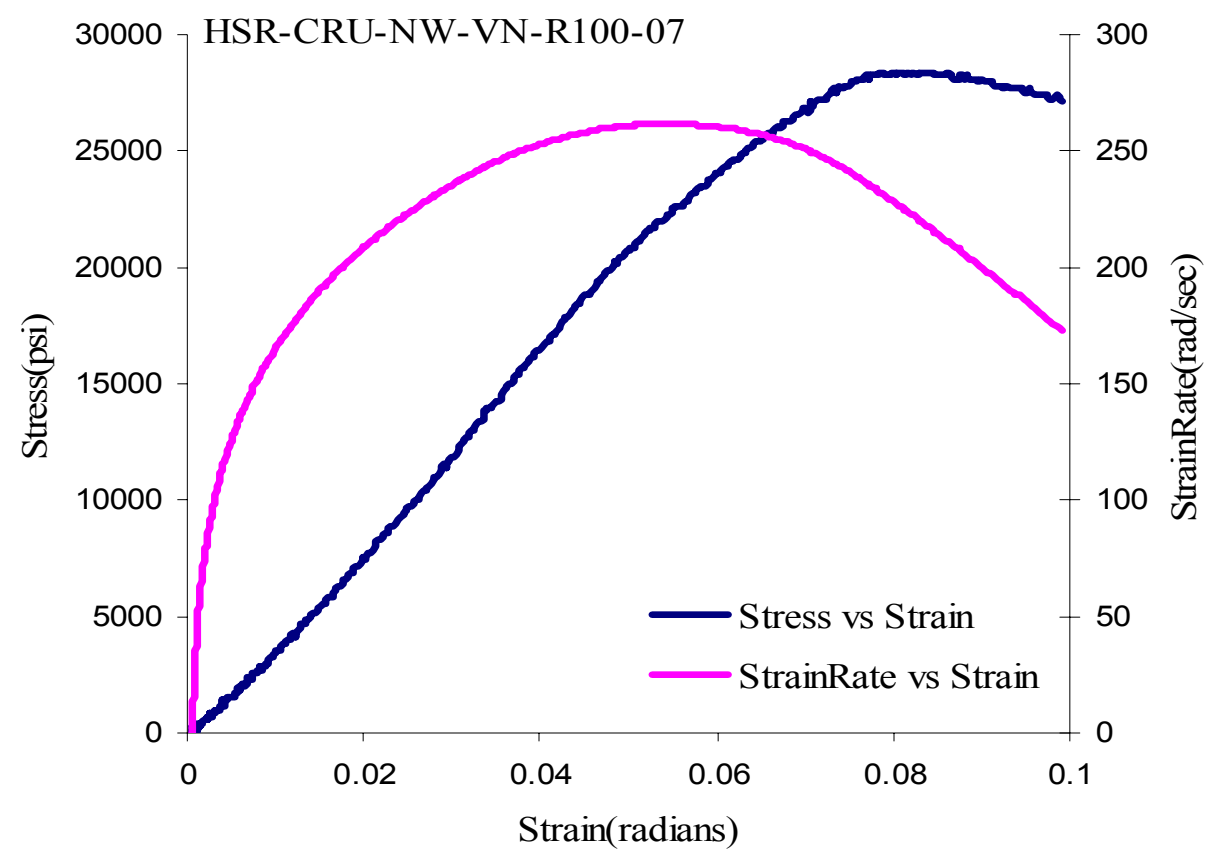

Figure 138. Stress-Strain and Strain Rate-Strain behavior of Newport Unitape at 100 in/s stroke rate

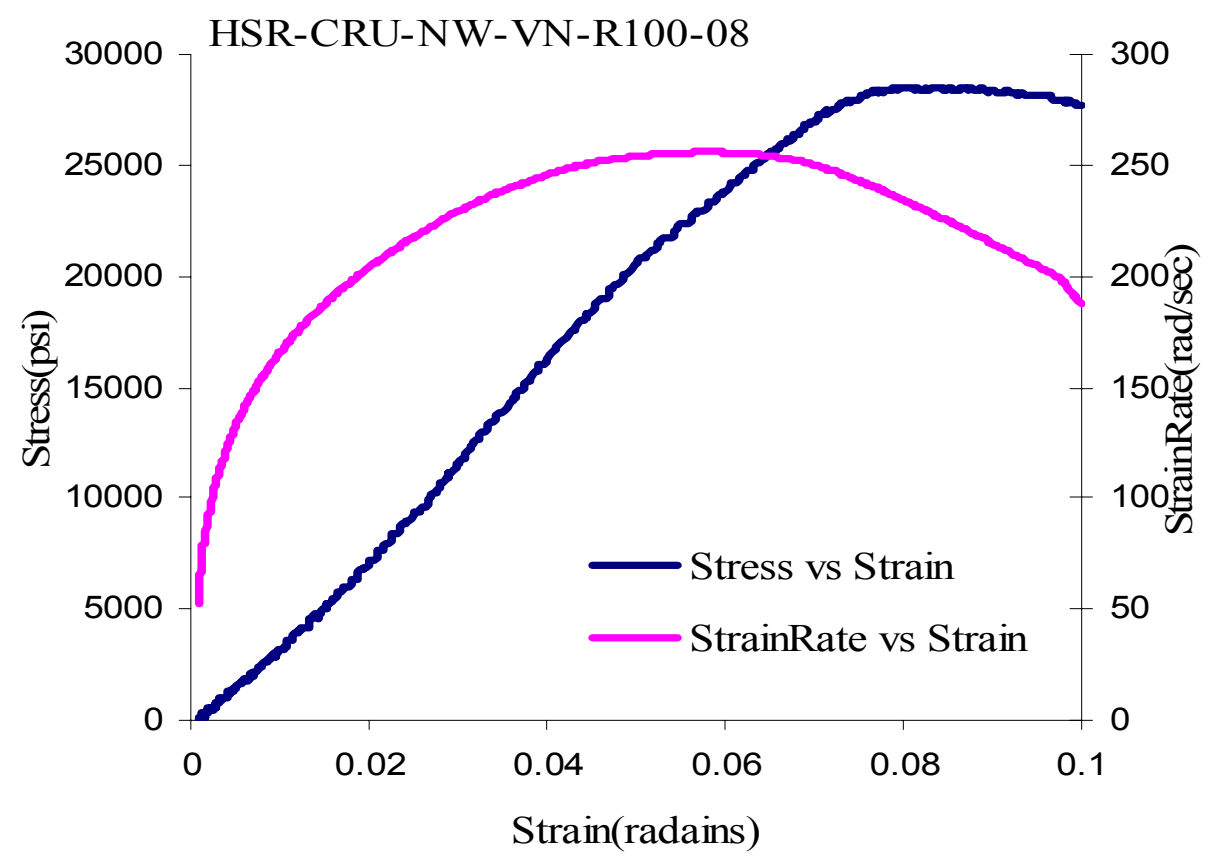

Figure 139. Stress-Strain and Strain Rate-Strain behavior of Newport Unitape at 100 in/s stroke rate 


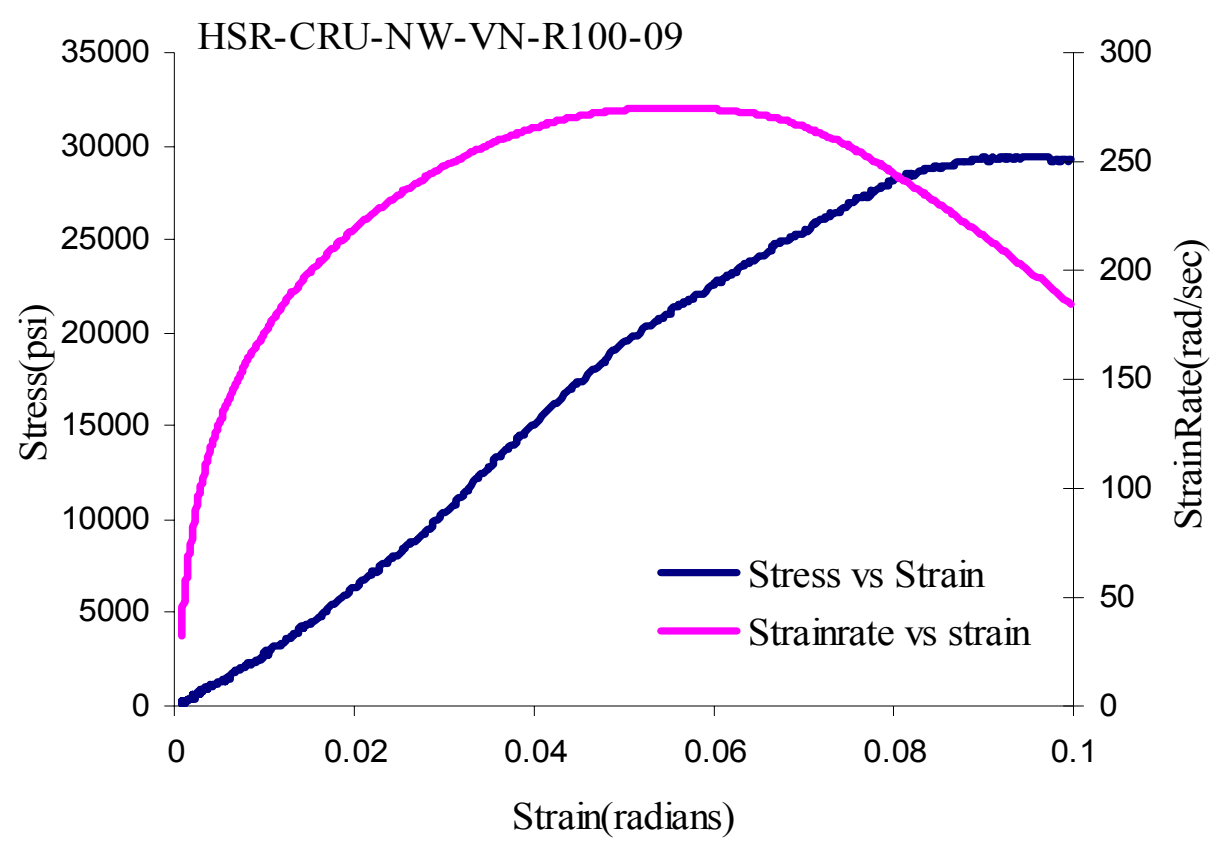

Figure 140. Stress-Strain and Strain Rate-Strain behavior of Newport Unitape at 100 in/s stroke rate

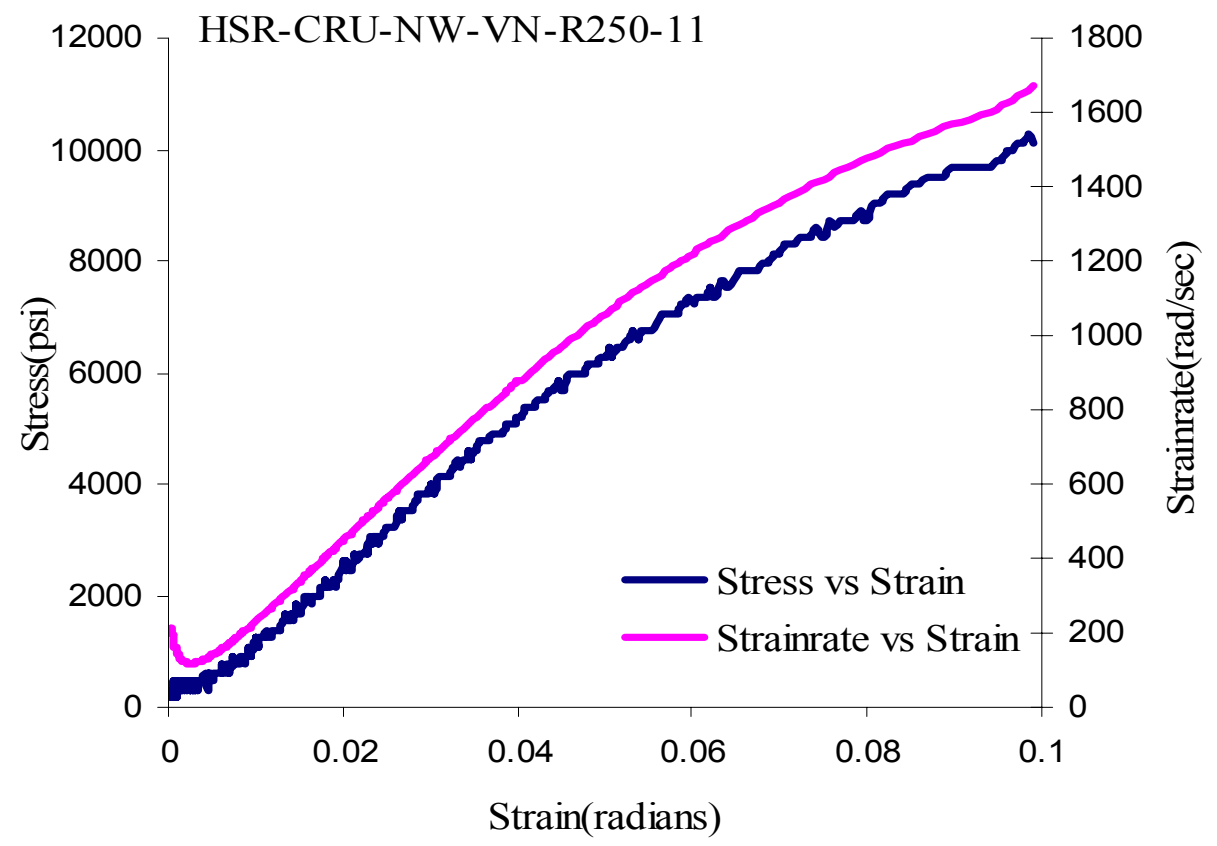

Figure 141. Stress-Strain and Strain Rate-Strain behavior of Newport Unitape at $250 \mathrm{in} / \mathrm{s}$ stroke rate 


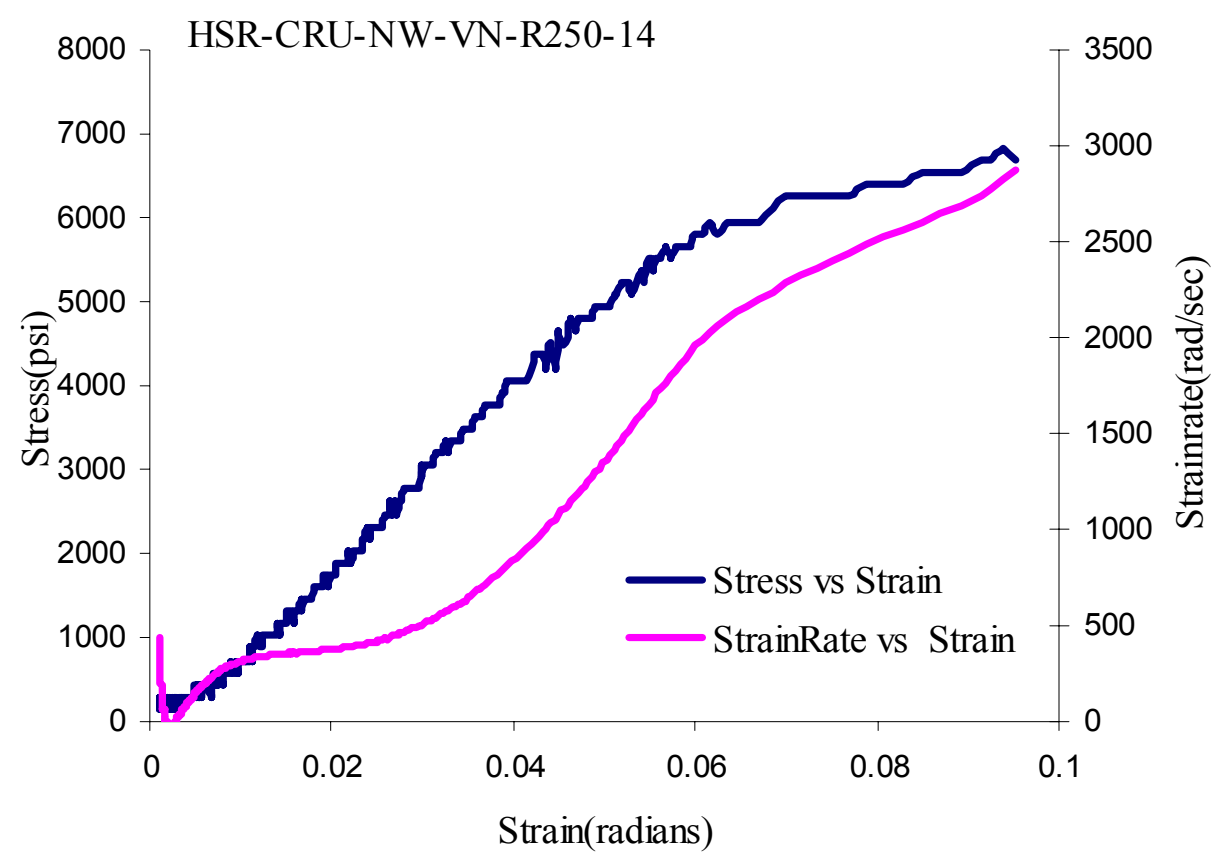

Figure 142. Stress-Strain and Strain Rate-Strain behavior of Newport Unitape at $250 \mathrm{in} / \mathrm{s}$ stroke rate

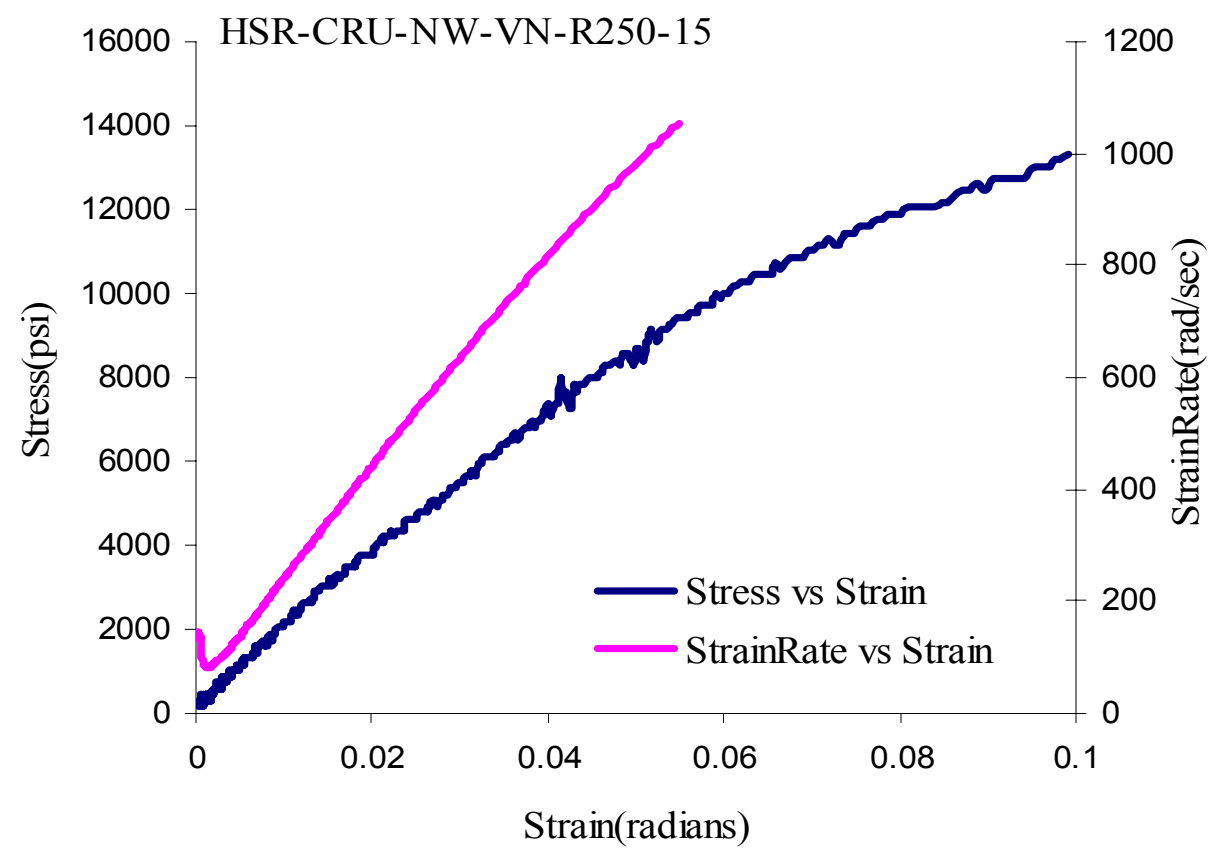

Figure 143. Stress-Strain and Strain Rate-Strain behavior of Newport Unitape at $250 \mathrm{in} / \mathrm{s}$ stroke rate 


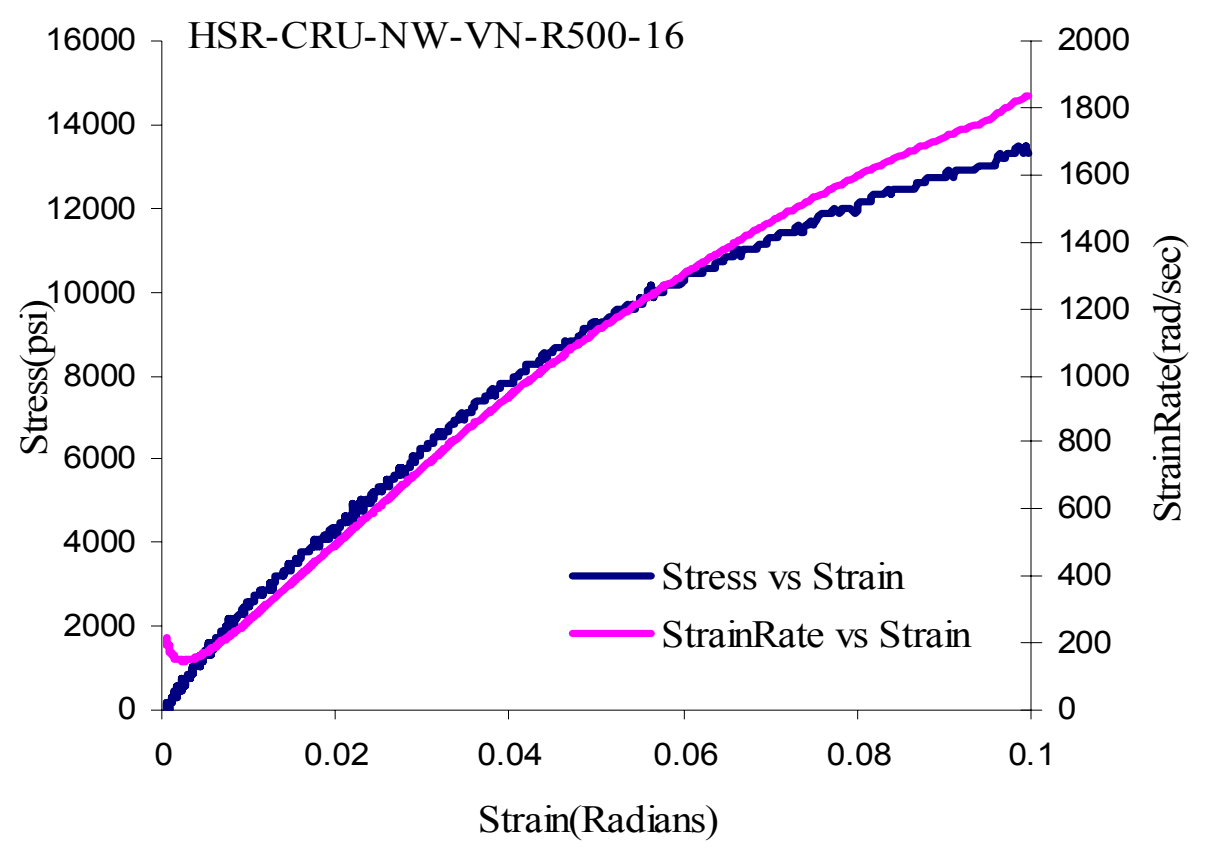

Figure 144. Stress-Strain and Strain Rate-Strain behavior of Newport Unitape at 500 in/s stroke rate

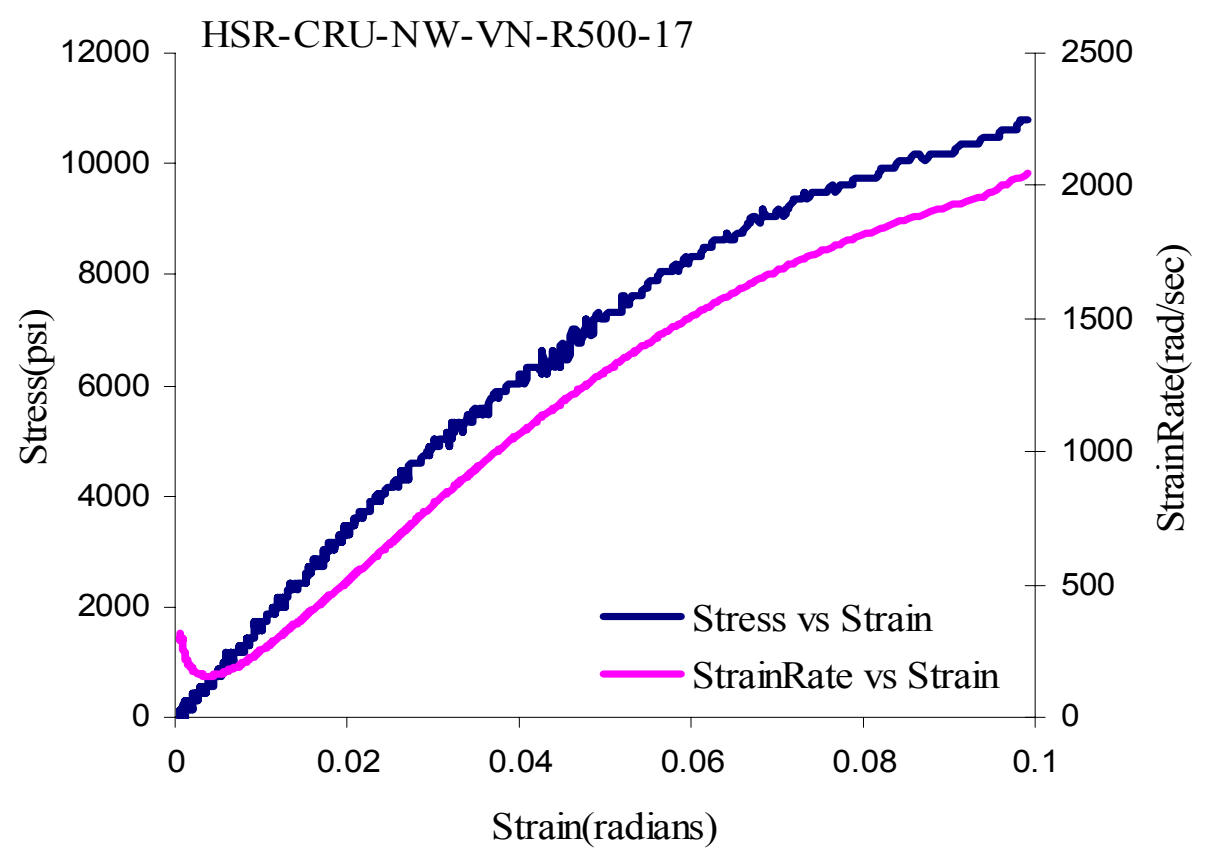

Figure 145. Stress-Strain and Strain Rate-Strain behavior of Newport Unitape at 500 in/s stroke rate 


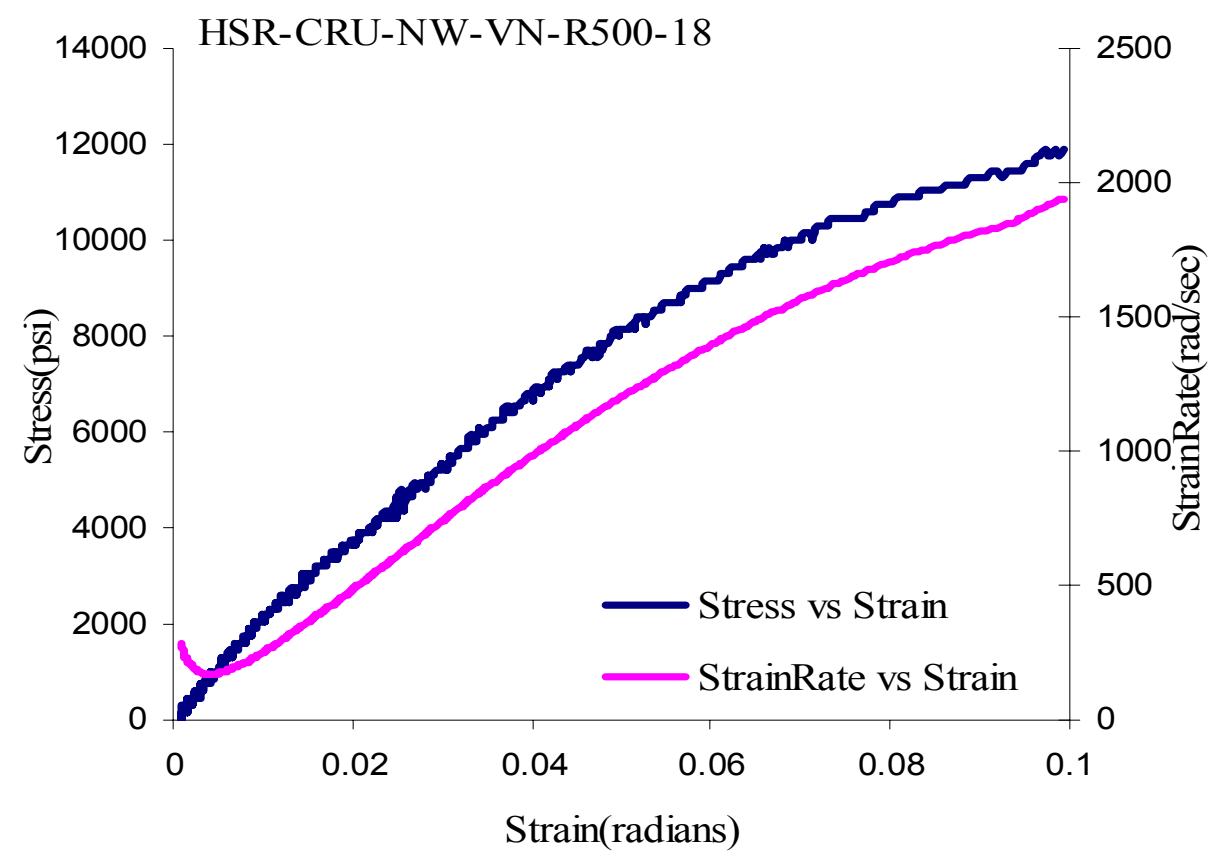

Figure 146. Stress-Strain and Strain Rate-Strain behavior of Newport Unitape at $500 \mathrm{in} / \mathrm{s}$ stroke rate

Failure Patterns of Newport NCT/321/G150 Unitape Specimens at different Stroke Rates

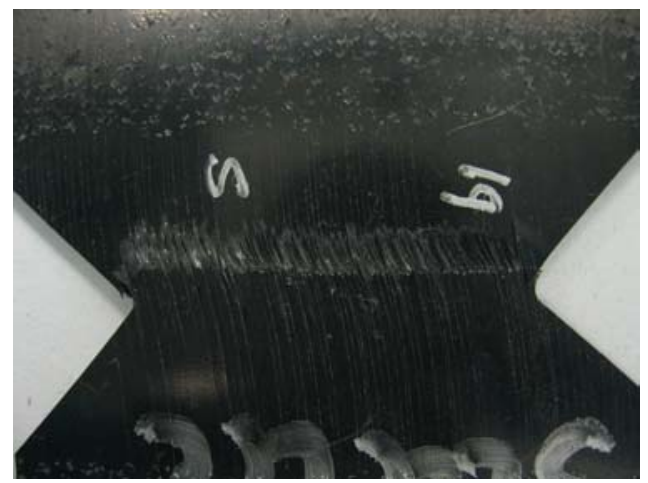

Figure 147. Failure mode of Newport Unitape material at $0.00083 \mathrm{in} / \mathrm{sec}$. 


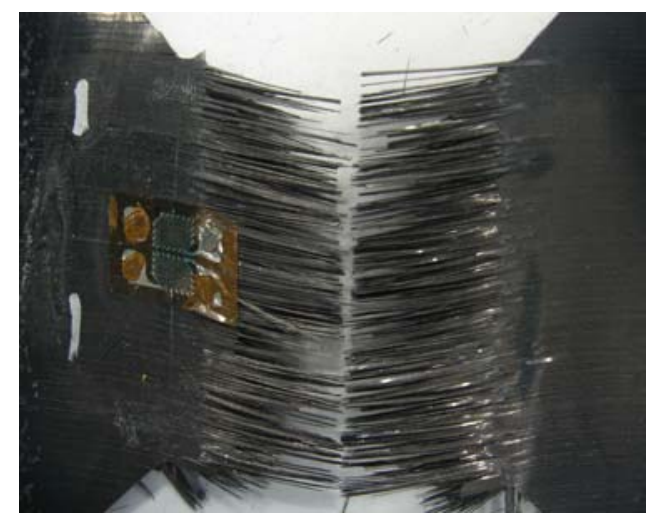

Figure 148. Failure mode of Newport Unitape material at $1 \mathrm{in} / \mathrm{sec}$.

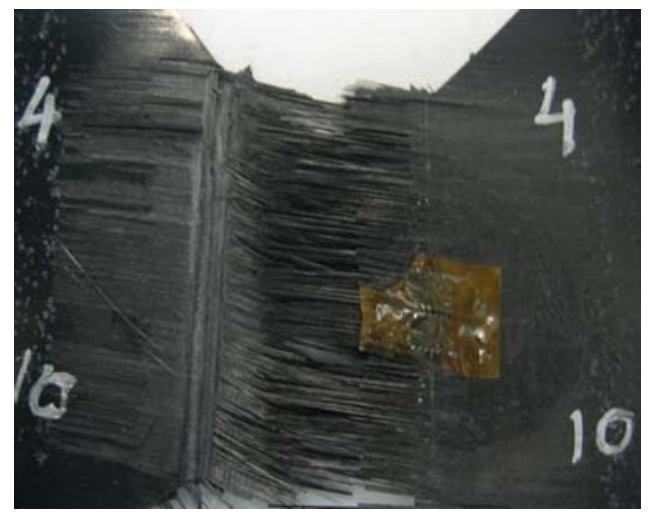

Figure 149. Failure mode of Newport Unitape material at $10 \mathrm{in} / \mathrm{sec}$.

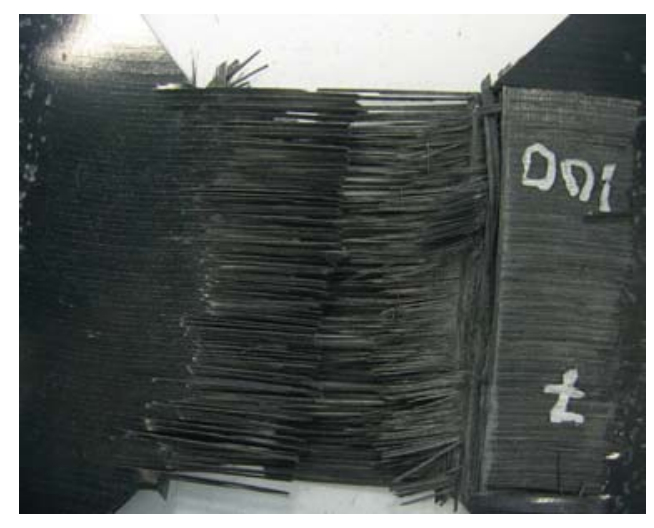

Figure 150. Failure mode of Newport Unitape material at $100 \mathrm{in} / \mathrm{sec}$. 


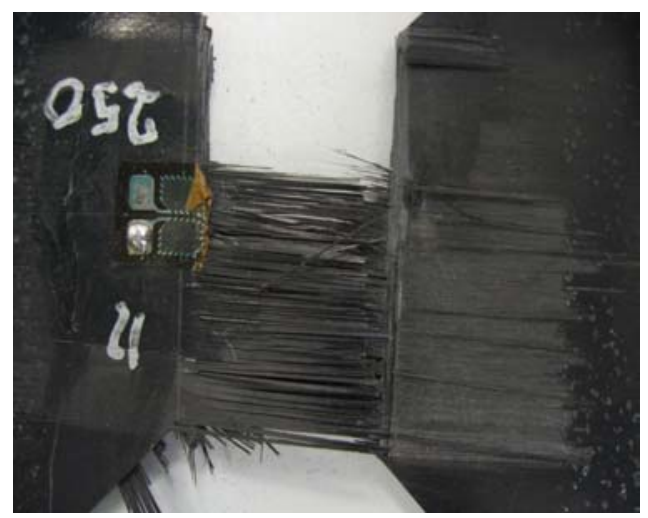

Figure 151. Failure mode of Newport Unitape material at $250 \mathrm{in} / \mathrm{sec}$.

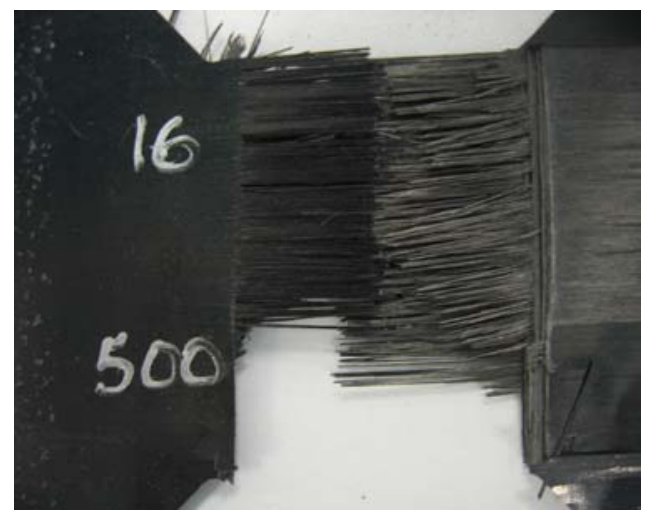

Figure 152. Failure mode of Newport Unitape material at $500 \mathrm{in} / \mathrm{sec}$. 
Table 12

Summary of test data for Newport Unitape material

\begin{tabular}{|c|c|c|c|c|c|}
\hline Specimen \# & $\begin{array}{l}\text { Average } \\
\text { Thickness } \\
\text { (in) }\end{array}$ & $\begin{array}{l}\text { Average Notch } \\
\text { to Notch Width } \\
\text { (in) }\end{array}$ & $\begin{array}{l}\text { Failure } \\
\text { Stress } \\
\left(\mathrm{lbf} / \mathrm{in}^{2}\right)\end{array}$ & $\begin{array}{l}\text { Max } \\
\text { Load } \\
(1 \mathrm{bs})\end{array}$ & $\begin{array}{l}\text { Average } \\
\text { Strain Rate } \\
(\mathrm{rad} / \mathrm{sec})\end{array}$ \\
\hline HSR-CRU-NW-VN-static-12 & 0.072 & 1.217 & 20626.01 & 1806.85 & 0.0016 \\
\hline HSR-CRU-NW-VN-static-19 & 0.072 & 1.223 & 17946.15 & 1578.07 & 0.0016 \\
\hline HSR-CRU-NW-VN-R1-01 & 0.073 & 1.220 & 22813.36 & 2018.55 & 1.21 \\
\hline HSR-CRU-NW-VN-R1-02 & 0.073 & 1.220 & 23814.61 & 2107.42 & 1.24 \\
\hline HSR-CRU-NW-VN-R1-03 & 0.072 & 1.218 & 22784.93 & 1993.16 & 0.93 \\
\hline HSR-CRU-NW-VN-R10-04 & 0.072 & 1.216 & 24191.89 & 2120.12 & 5.64 \\
\hline HSR-CRU-NW-VN-R10-05 & 0.073 & 1.215 & 24049.90 & 2132.81 & 6.36 \\
\hline HSR-CRU-NW-VN-R10-06 & 0.073 & 1.217 & 24329.17 & 2170.90 & 9.31 \\
\hline HSR-CRU-NW-VN-R100-07 & 0.073 & 1.222 & 42234.76 & 3757.81 & 191.02 \\
\hline HSR-CRU-NW-VN-R100-08 & 0.072 & 1.221 & 40339.12 & 3554.69 & 204.63 \\
\hline HSR-CRU-NW-VN-R100-09 & 0.071 & 1.221 & 41681.76 & 3618.16 & 213.23 \\
\hline HSR-CRU-NW-VN-R250-11 & 0.071 & 1.222 & 24389.95 & 2018.55 & 538.42 \\
\hline HSR-CRU-NW-VN-R250-14 & 0.072 & 1.219 & 24255.51 & 2120.12 & 508.00 \\
\hline HSR-CRU-NW-VN-R250-15 & 0.072 & 1.224 & 24069.93 & 2107.42 & 483.64 \\
\hline HSR-CRU-NW-VN-R500-16 & 0.072 & 1.224 & 25471.77 & 2234.38 & 621.83 \\
\hline HSR-CRU-NW-VN-R500-17 & 0.072 & 1.224 & 22694.48 & 2005.86 & 655.52 \\
\hline HSR-CRU-NW-VN-R500-18 & 0.072 & 1.222 & 23215.56 & 2031.25 & 649.18 \\
\hline
\end{tabular}

